$10 / 3 /-94 \mathrm{~J} \delta(1)$
SANDIA REPORT

SAND92-1382 - UC-721

Unlimited Release

Printed November 1993

\title{
Expert Judgment on Markers to Deter Inadvertent Human Intrusion into the Waste Isolation Pilot Plant
}

\author{
Kathleen M. Trauth, Stephen C. Hora, Robert V. Guzowski
}

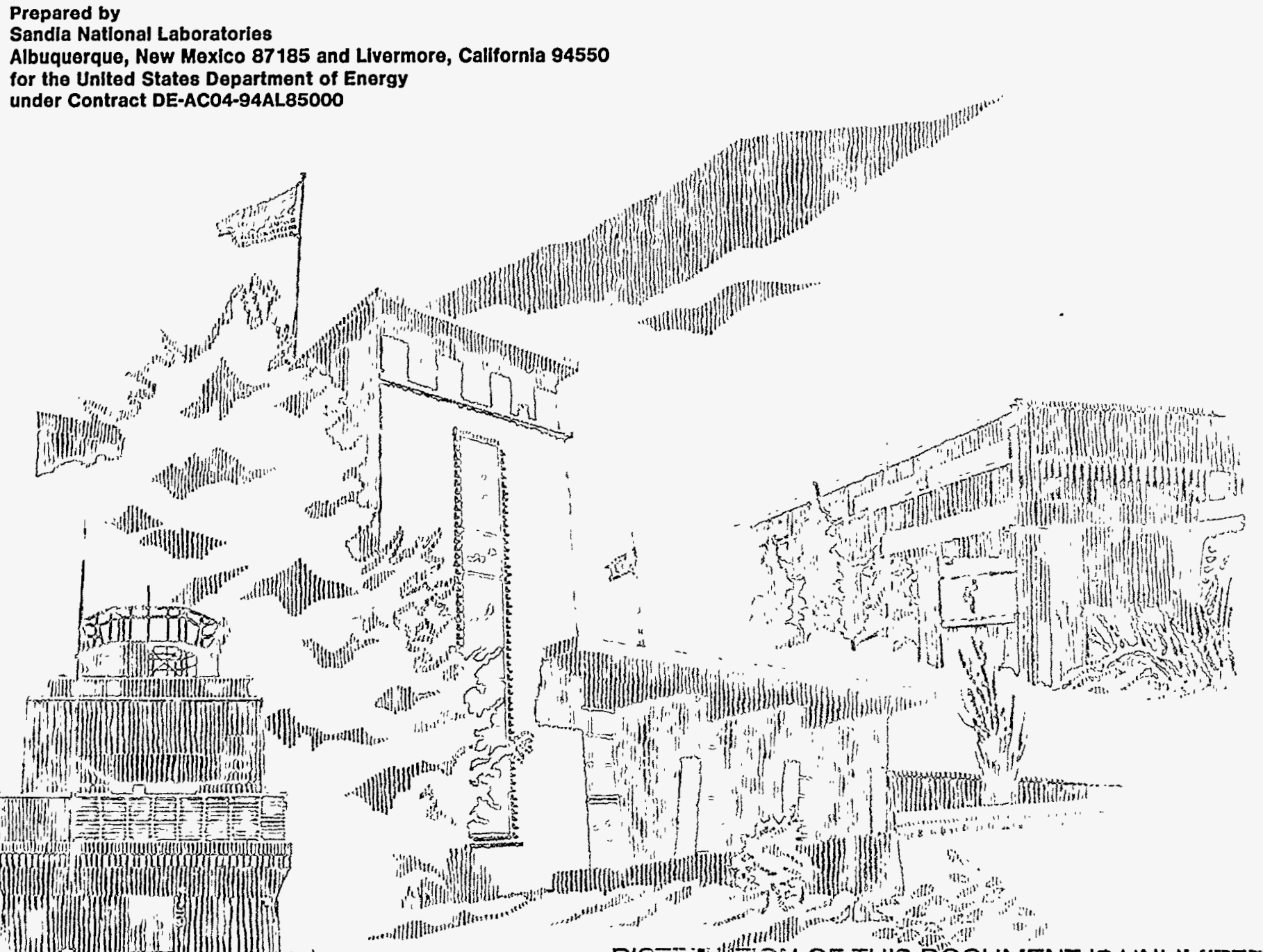


Issued by Sandia National Laboratories, operated for the United States Department of Energy by Sandia Corporation.

NOTICE: This report was prepared as an account of work sponsored by an agency of the United States Government. Neither the United States Government nor any agency thereof, nor any of their employees, nor any of their contractors, subcontractors, or their employees, makes any warranty, express or implied, or assumes any legal liability or responsibility for the accuracy, completeness, or usefulness of any information, apparatus, product, or process disclosed, or represents that its use would not infringe privately owned rights. Reference herein to any specific commercial product, process, or service by trade name, trademark, manufacturer, or otherwise, does not necessarily constitute or imply its endorsement, recommendation, or favoring by the United States Government, any agency thereof or any of their contractors or subcontractors. The views and opinions expressed herein do not necessarily state or reflect those of the United States Government, any agency thereof or any of their contractors.

Printed in the United States of America. This report has been reproduced directly from the best available copy.

Available to DOE and DOE contractors from

Office of Scientific and Technical Information

PO Box 62

Oak Ridge, TN 37831

Prices available from (615) 576-8401, FTS 626-8401

Available to the public from

National Technical Information Service

US Department of Commerce

5285 Port Royal Rd

Springfield, VA 22161

NTIS price codes

Printed copy: A15

Microfiche copy: A01 


\section{DISCLAIMER}

Portions of this document may be illegible electronic image products. Images are produced from the best available original document. 
SAND92-1382

Distribution

Unlimited Release

Category UC-721

Printed November 1993

\title{
Expert Judgment on Markers to Deter Inadvertent Human Intrusion into the Waste Isolation Pilot Plant
}

\author{
Kathleen M. Trauth, Stephen C. Hora ${ }^{1}$, and Robert V. Guzowski ${ }^{2}$ \\ Sandia National Laboratories \\ Albuquerque, NM 87185
}

\begin{abstract}
Sandia National Laboratories (SNL) convened an expert panel to develop design characteristics for permanent markers and to judge the efficacy of the markers in deterring inadvertent human intrusion in the Waste Isolation Pilot Plant (WIPP). The WIPP, located in southeastern New Mexico, is designed to demonstrate the safe disposal of transuranic (TRU) radioactive wastes generated by the United States Department of Energy (DOE) defense programs. The DOE must evaluate WIPP compliance with the Environmental Protection Agency (EPA) regulation Environmental Standards for the Management and Disposal of Spent Nuclear Fuel, High-Level and Transuranic Radioactive Wastes ( 40 CFR Part 191, Subpart B); this EPA regulation requires: "Disposal sites shall be designated by the most permanent markers, records, and other passive institutional controls practicable to indicate the dangers of the wastes and their location" (Federal Register $50: 38086 \mathrm{c})$. The period of regulatory, concern is 10,000 years.

The expert panel identified basic principles to guide current and future marker development efforts: (1) the site must be marked, (2) message(s) must be truthful and informative, (3) multiple components within a marker system, (4) multiple means of communication (e.g., language, pictographs, scientific diagrams), (5) multiple levels of complexitiy within individual messages on individual marker system elements, (6) use of materials with little recycle value, and (7) international effort to maintain knowledge of the locations and contents of nuclear waste repositories. The efficacy of the markers in deterring inadvertent human intrusion was estimated to decrease with time, with the probability function varying with the mode of intrusion (who is intruding and for what purpose) and the level of technological development of the society. The development of a permanent, passive marker system capable of surviving and remaining interpretable for 10,000 years will require further study prior to implementation.
\end{abstract}

1 University of Hawaii at Hilo

2 Science Applications International Corporation

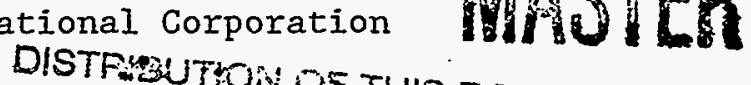




\section{ACKNOWLEDGMENTS}

The authors wish to thank the individuals who served on the Marker Development Panel.

- Team A. Dieter G. Ast (Cornell University), Michael Brill (Buffalo Organization for Social and Technological Innovation, Inc.), Maureen F. Kaplan (Eastern Research Group, Inc.), Ward H. Goodenough (University of Pennsylvania), Frederick J. Newmeyer (University of Washington), and Woodruff T. Sullivan, III (University of Washington).

- Team B. Victor R. Baker (University of Arizona), Frank D. Drake (University of California at Santa Cruz), Ben R. Finney (University of Hawaii at Manoa), David B. Givens (American Anthropological Association), Jon Lomberg (independent artist, designer, and writer), Louis Narens (University of California at Irvine), and Wendell Williams (Case Western Reserve University).

Thanks go to members of the Futures Panel who discussed their team's efforts and recommendations with the Markers panel-Michael Baram (Boston Team), Martin Pasqualetti (Southwest Team), Dan Reicher (Washington A Team), and Maris Vinovskis (Washington B Team). Thanks also go to the Sandia Staff who presented material to the experts--D. Richard Anderson (6342), Marilyn Gruebel (Tech Reps, Inc.), Peter Swift (6342), and Wendell Weart (6303). Virginia Gilliland and Molly Minahan (Technical Writers), Debbie Marchand and Hawaii Olmstead (Illustrations), Susan Gill (Technical Editor), Steve Tullar (Production), and Debra Rivard and the Word Processing Department of Tech Reps, Inc. provided support in conducting the meetings and documenting the results.

The authors would also like to thank the technical reviewers, Marilyn Gruebel (Tech Reps, Inc.) and Erik Webb (6331) for their useful comments. 


\section{PREFACE}

This SAND report was prepared from information presented by a panel of experts expressing judgments about the design and efficacy of markers to deter inadvertent human intrusion into the Waste Isolation Pilot Plant (WIPP). Appendices $F$ and $G$ were written by the panelists. The authors consolidated and utilized these appendices in preparing the body of the report. The individual reports are reprinted as received by the project coordinator except for (1) correcting typographical errors, (2) editing for internal format consistency, (3) renumbering, repositioning, and captioning figures, (4). updating the table of contents to be in line with the previous changes, and (5) changing the text in accordance with answers to a number of questions that were addressed to the individual teams about their reports as written. The members of the expert panel reviewed a draft copy of the report and the updated versions of Appendices $F$ and $G$, and responded to the questions provided.

The panel of experts made their judgments based on current (as of November 1991) information from disciplines pertinent to markers and about the WIPP Project itself. A final decision on marker system design and placement will be based on all information that is available to the WIPP Project at the time the decision is made. 


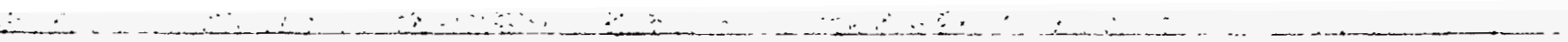




\section{CONTENTS}

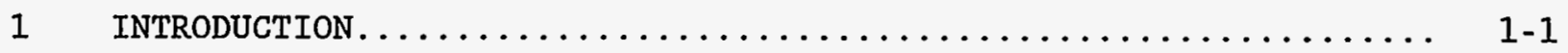

1.1 Regulatory Requirement for Markers................ 1-5

1.2 Background............................... $1-8$

1.2 .1 Future Societies..................... 1-8

1.2 .2 Marker Development.................... 1-11

1.3 Purposes of the study........................ 1-12

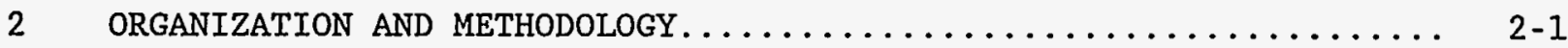

2.1 Using Expert Judgment........................ $2-1$

2.2 Expert-Judgment Panel....................... $2-2$

2.2.1 Decision to Use an Expert-Judgment Pane1........... 2-2

2.2.2 Development of the Issue Statement............. 2-2

2.2.3 Selection of Experts......................... $2-4$

2.2.3.1 Nomination Process................. 2-4

2.2.3.2 Selection Advisory Committee........... 2-5

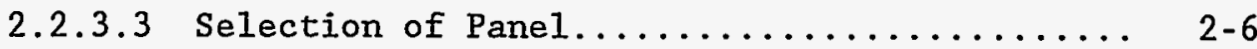

2.2.4 Panel Deliberations..................... 2-6

3 RECOMMENDED DESIGN CHARACTERISTICS $\ldots \ldots \ldots \ldots \ldots \ldots \ldots \ldots \ldots \ldots \ldots$

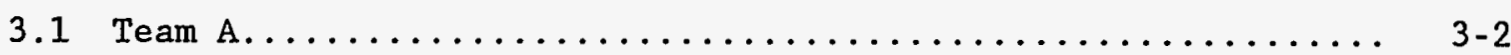

3.1 .1 Basic Premises........................ 3-2

3.1 .2 Assumptions/Bases...................... 3-2

3.1.3 Message Levels and Media................... 3-3

3.1 .4 Marker System Components.................. $3-4$

3.1 .5 Other Design Requirements................. $3-5$

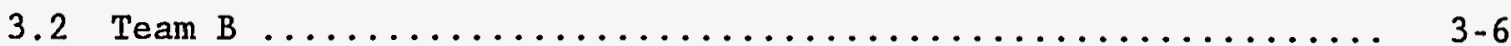

3.2.1 Basic Premises......................... 3-6

3.2 .2 Assumptions/Bases......................... $3-8$

3.2.3 Message Levels and Media................... 3-8

3.2.4 Marker System Components.................. 3-9

4 PRINCIPLES OF MARKING . . . . . . . .

4.I Architectural-Design Principles.................. 4-1 
4.2 Linguistic Principles....................... 4-5

4.3 Material-Properties Principles.................. $4-5$

4.4 Message-Level Principles..................... 4-6

$5 \quad$ PROBABILITY ELICITATION.......................... $5-1$

5.1 Persistence of Markers....................... $5-1$

5.2 Interpretability of Messages................... $5-2$

5.3 Gonclusions and Implementation.................. $5-9$

REFERENCES ................................. $6-1$

Appendix A: Issue Statement for the Development of Markers to Deter Inadvertent Human Intrusion into the Waste Isolation Pilot Plant........................... A -1

Appendix B: Letter Requesting Nominations.................. B-1

Appendix C: List of Those to Whom Requests for Nominations Were Sent.. C-1

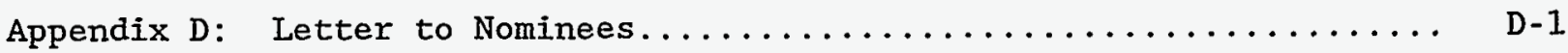

Appendix E: Expert Panel Selection Criteria................ E-1

Appendix F: Team A Report: Marking the Waste Isolation Pilot Plant for 10,000 Years...................... F-1

Appendix G: Team B Report: The Development of Markers to Deter Inadvertent Human Intrusion into the Waste Isolation

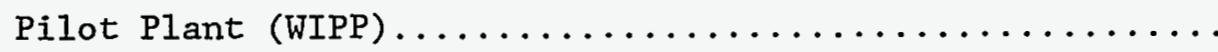




\section{Figures}

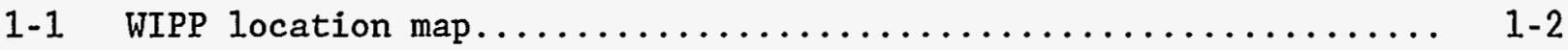

1-2 Proposed WIPP repository, showing both TRU-waste disposal areas and experimental areas......................... 1-3

1-3 Generalized WIPP stratigraphy................... 1-4

1-4 Artist's concept of the WIPP disposal system showing the controlled area and accessible environment for $40 \mathrm{CFR} 191$, Subpart B, and the repository/shaft system............. 1-7

\section{Tables}

2-1 Marker Development Panel........................ 2-7

4-1 Marker System Components........................ 4-2

5-1 Probabilities of the Marker System Persisting - Team A....... 5-2

5-2 Consensus Probabilities of the Marker System Persisting Team B..................................... $5-3$

5-3 Probability of Correct Interpretation of Message--Drilling for Water as Mode of Intrusion......................... 5-4

5-4 Probability of Correct Interpretation of Message--Mineral Exploration as Mode of Intrusion.................. 5-5

5-5 Probability of Correct Interpretation of Message--Drilling for Disposal Wells as Mode of Intrusion................. 5-6

5-6 Probability of Correct Interpretation of Message--Archaeological Investigation as Mode of Intrusion................ 5-7

5-7 Probability of Correct Interpretation of Message--Scientific Investigation as Mode of Intrusion................. 5-8 


\section{INTRODUCTION}

Expert elicitation was used to determine the potential for markers to deter inadvertent human intrusion by future generations into the Waste Isolation Pilot Plant (WIPP). Specific goals were to obtain information about marker designs and message formats that will remain in existence and interpretable for the required time period of regulatory concern, and to estimate the effectiveness of specific marker designs in deterring intrusion and communicating a warning to future generations about the location and nature of the waste buried at the WIPP: The assumption was made that when individuals know what materials are buried in the area and the dangers of intruding into the material, they will not do so. This effort was undertaken by the Performance Assessment Department at Sandia National Laboratories (SNL).

This effort to communicate a warning to deter inadvertent human intrusion into a repository is necessary because of the hazardous materials that are planned for disposal in the WIPP facility. The radioactively contaminated waste should be isolated from the biosphere until the risks posed by possible releases are acceptably small. In order to accomplish this isolation, knowledge of the location and the nature of the wastes must be maintained and passed on to successive future societies. Markers are physical structures (such as earthworks, stone monoliths, and rock cairns) that are capable of carrying the intended message for a long period of time. The message is the means of communicating with whatever future societies may exist.

The WIPP was authorized by Public Law 96-164 (1979) as a research and development facility "to demonstrate the safe disposal of radioactive wastes resulting from the defense activities and programs of the United States exempted from regulation by the Nuclear Regulatory Commission..." Physically, the WIPP is a facility located approximately 26 miles $(42 \mathrm{~km})$ east of Carlsbad, New Mexico (Figure 1-1). The planned repository is schematically shown in Figure 1-2. Some of the experimental areas have already been mined at $2157 \mathrm{ft}(657 \mathrm{~m})$ below the surface, within the beddedsalt Salado Formation (Figure 1-3). If the WIPP is approved as a disposal facility, it will accept laboratory and production waste contaminated with transuranic elements produced by the nuclear-weapons program. Transuranic (TRU) waste is defined for regulatory purposes as waste contaminated with radionuclides having an atomic number greater than 92 , a half-life greater than 20 years, and a concentration greater than $100 \mathrm{nCi} / \mathrm{g}$. In addition to TRU waste, lead, radium, thorium, uranium, and contaminants with half-lives less than 20 years are expected to be disposed of at the WIPP. While the WIPP's primary mission is for the disposal of radioactive wastes, the nature 


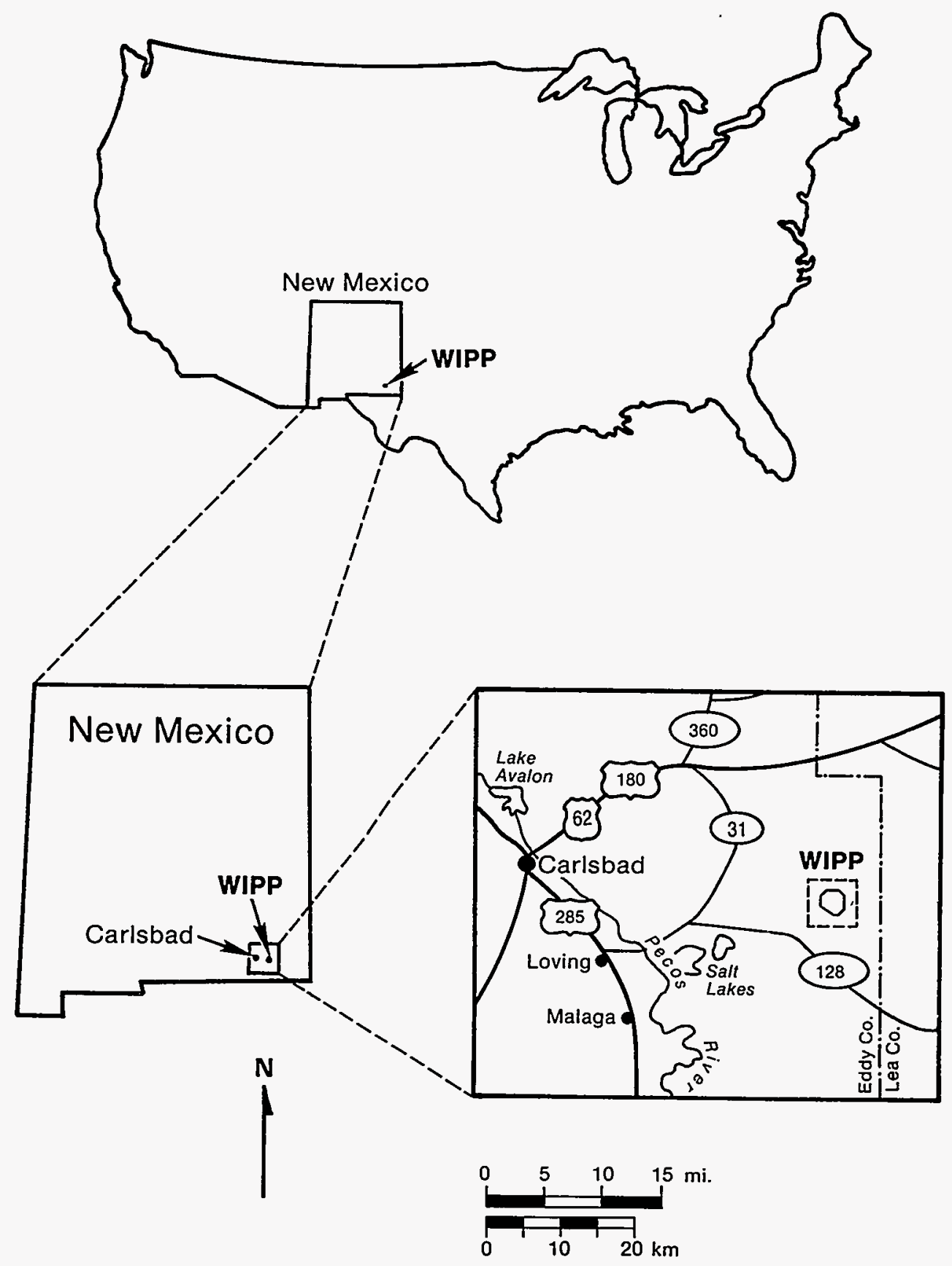

TRI-6342-223-1

Figure 1-1. WIPP location map (after Bertram-Howery and Hunter, 1989). 
1. Introduction

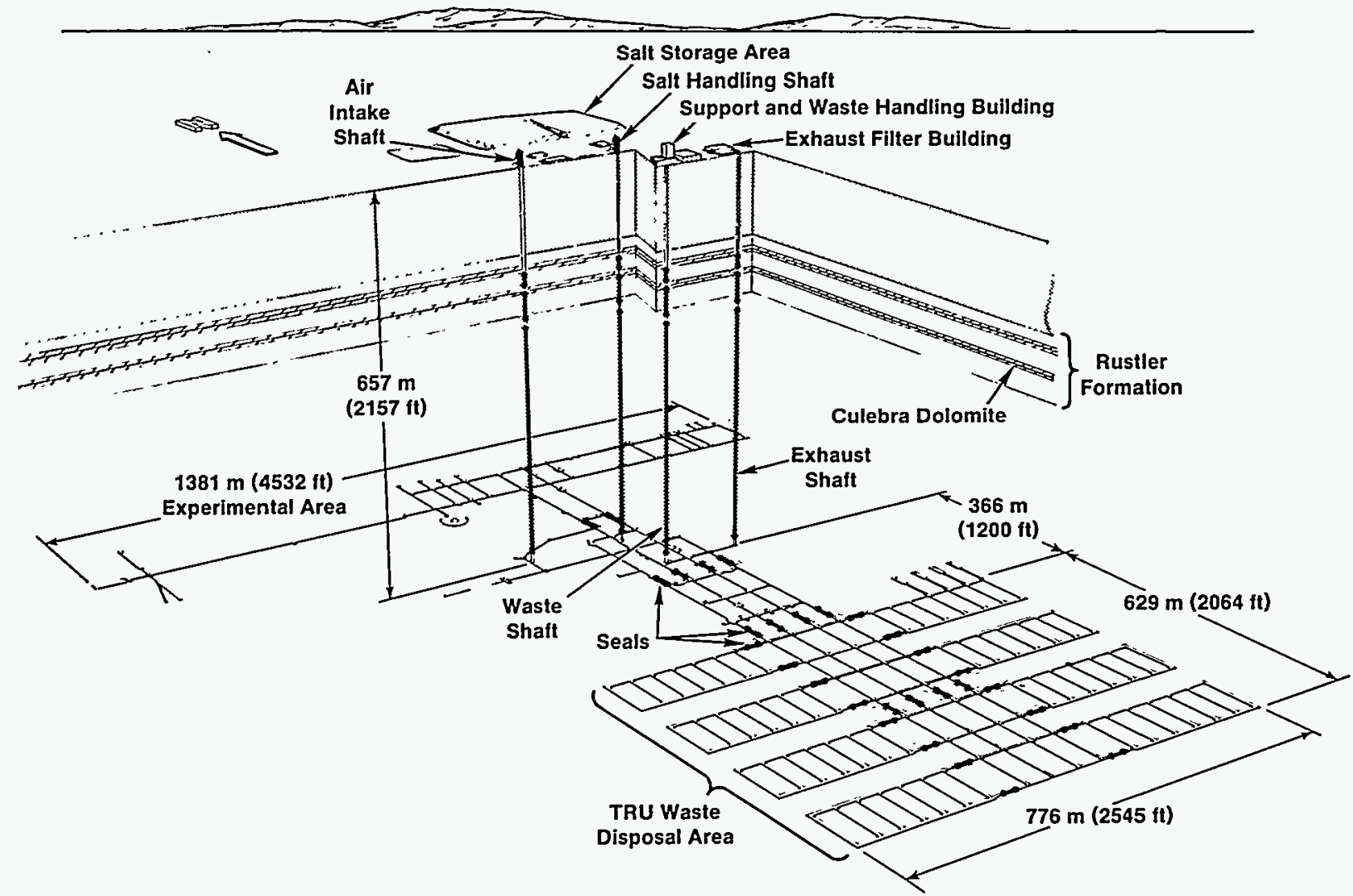

TRI-6346-59-10

Figure 1-2. Proposed WIPP repository, showing both TRU-waste disposal areas and experimental areas (after Waste Management Technology Dept., 1987). 

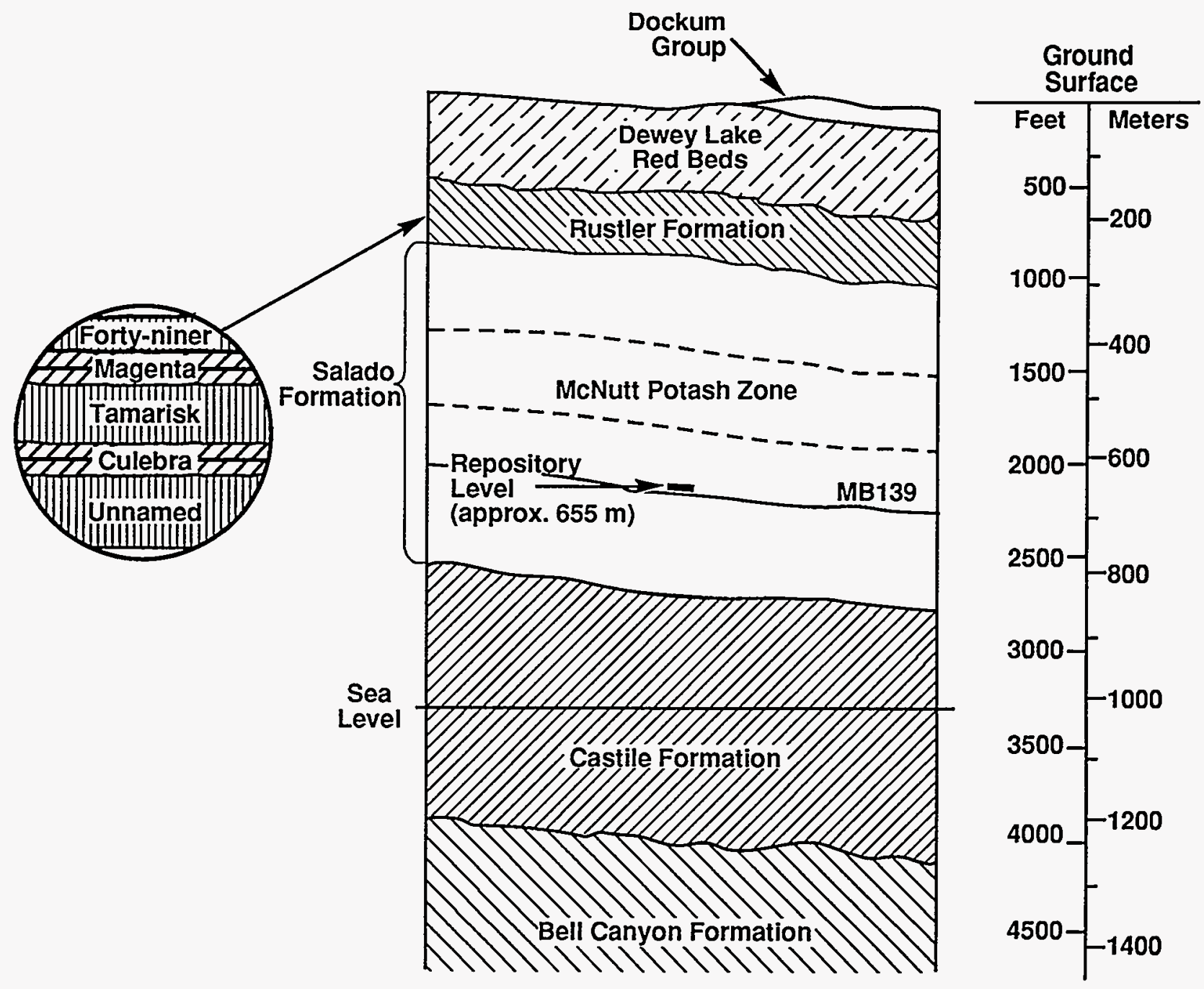

Figure 1-3. Generalized WIPP stratigraphy (after Rechard, 1989; based on U.S. DOE, 1980). 
of the waste is such that some hazardous materials may contaminate the radioactive waste.

\subsection{Regulatory Requirement for Markers}

The disposal of nuclear waste at the WIPP is governed by the Environmental Protection Agency's (EPA's) Environmental Standards for the Management and Disposal of Spent Nuclear Fuel, High-Level and Transuranic Radioactive Wastes ( $40 \mathrm{CFR}$ Part 191; EPA, 1985), referred to herein as the Standard. Subpart A governs the operation of a repository prior to closure and will not be discussed further in this report. Subpart B governs the operation of a repository after closure and for the entire regulatory period of 10,000 years. Subpart B was vacated and remanded to the EPA by the US Court of Appeals for the First Circuit in 1987. Through the Second Modification to the Consultation and Cooperation Agreement (U.S. DOE and State of New Mexico, 1981), studies regarding the performance of the WIPP will continue under the provisions of the remanded standard until a new Standard is promulgated.

The Containment Requirements ( $\$ 191.13)$ of the Standard set limits for the cumulative release of radionuclides to the accessible environment. The cumulative release limits are couched in terms of the magnitude of a potential release and the probability of its occurrence. Such potential releases are to be calculated during the course of a performance assessment. The performance assessments for the WIPP are conducted by the Performance Assessment Department at SNL. A performance assessment is defined in the Standard $(\$ 191.12(\mathrm{q}))$ as a process that:

(1) Identifies the processes and events that might affect the disposal system; (2) examines the effects of these processes and events on the performance of the disposal system; and (3) estimates the cumulative releases of radionuclides, considering the associated uncertainties, caused by all significant processes and events. These estimates shall be incorporated into an overall probability distribution of cumulative release to the extent practicable.

Releases are evaluated within boundaries determined by several definitions. Accessible environment is defined in the Standard ( $\$ 191.12(\mathrm{k})$ ) as: "(1) The atmosphere; (2) land surfaces; (3) surface waters; (4) oceans; and (5) all of the lithosphere that is beyond the controlled area." The controlled area is defined in the Standard $(\$ 191.12(\mathrm{~g}))$ as:

(1) A surface location, to be identified by passive institutional controls, that encompasses no more than 100 square kilometers and extends horizontally no more than five kilometers in any direction 
from the outer boundary of the original location of the radioactive wastes in a disposal system; and (2) the subsurface underlying such a surface location.

The accessible environment and controlled area are shown in Figure 1-4.

The Assurance Requirements ( $\$ 191.14)$ state, in part, that:

Disposal sites shall be designated by the most permanent markers, records, and other passive institutional controls practicable to indicate the dangers of the wastes and their location.

The term "disposal site" (as here quoted from Subpart B of the Standard) is interpreted to mean the controlled area. In the WIPP Land Withdrawal Act (WIPP LWA) (Public Law 102-579, approved October 30, 1992), Congress withdrew 16 square miles of land "...from all forms of entry, appropriation, and disposal under the public land laws..."; transferred jurisdiction from the Secretary of the Department of the Interior to the Secretary of the Department of Energy; and stated that "Such lands are reserved for the use of the Secretary [of the Department of Energy] for the construction, experimentation, operation, repair and maintenance, disposal, shutdown, monitoring, decommissioning, and other authorized activities associated with the purposes of WIPP..." The land withdrawal boundary is shown in Figure 1-4. Performance assessment calculations currently use the land withdrawal boundary to assess compliance with the 10,000-year release limits.

The Standard defines passive institutional control in $\$ 191.12(\mathrm{e})$ as:

(1) Permanent markers placed at a disposal site, (2) public records and archives, (3) government ownership and regulations regarding land or resource use, and (4) other methods of preserving knowledge about the location, design, and contents of a disposal system.

As explained in the Supplementary Information to the Standard, the Assurance Requirements are included in order to address the fact that there are many uncertainties in the analysis of releases to the accessible environment over the 10,000 years of regulatory concern. The requirement for additional measures to improve the operation of a repository is a means to address these uncertainties.

The second context for the use of markers follows from the previous requirement ( $\$ 191.14)$. Given the fact that markers must be used for a nuclear waste repository, EPA's Guidance to the Standard allows credit to be taken for the impact of markers in reducing the probability of inadvertent human intrusion (although it can never be assumed to be zero): 


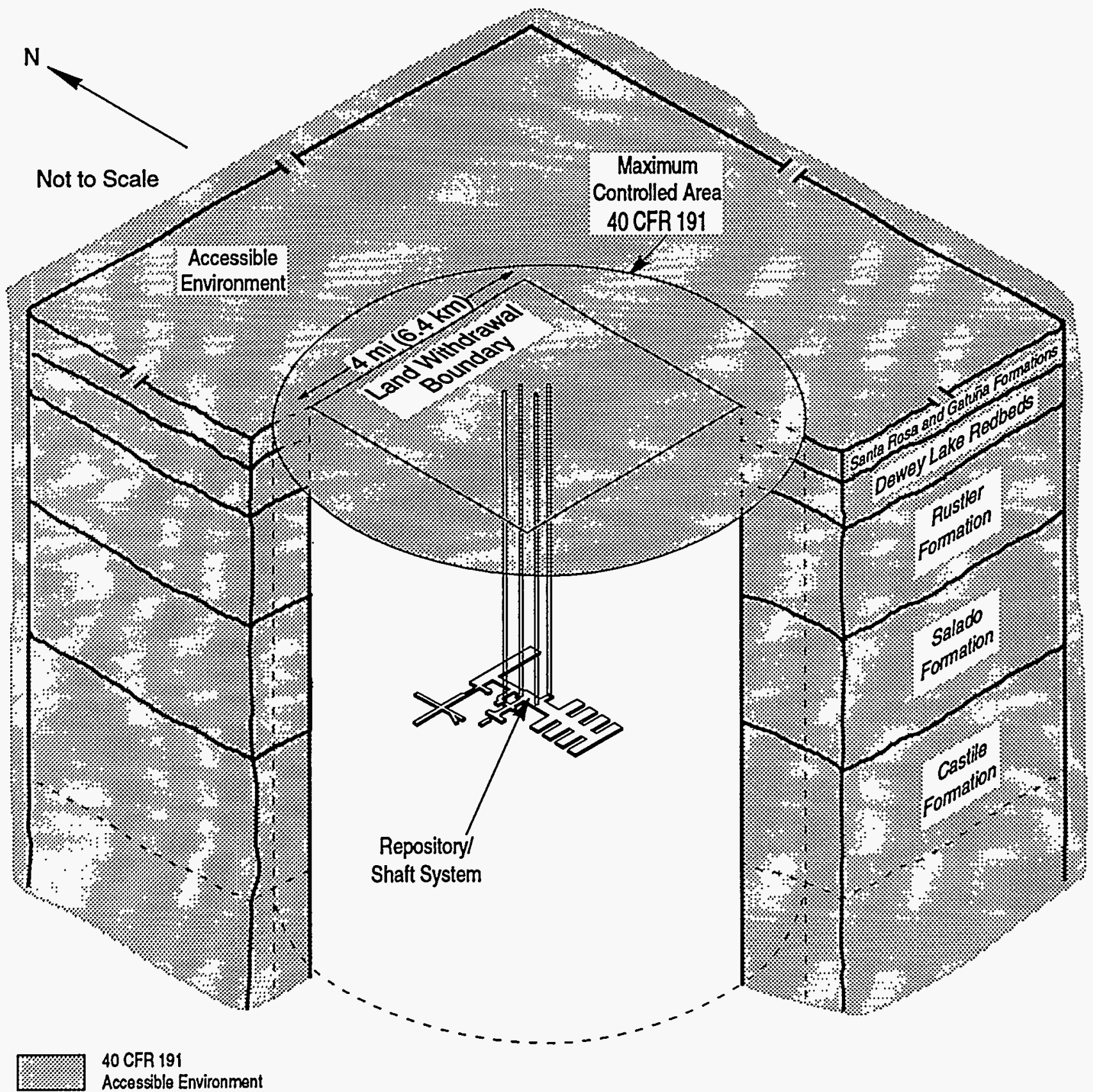

$\operatorname{TR} 1-6330-7-12$

Figure 1-4. Artist's concept of the WIPP disposal system showing the controlled area and accessible environment for 40 CFR 191, Subpart B, and the repository/shaft system. The repository/shaft system scale is exaggerated. On the land surface, the land-withdrawal boundary is shown at the same scale as the maximum extent of the controlled area (modified from Bertram-Howery and Hunter, 1989). 
The Agency assumes that, as long as such passive institutional controls endure and are understood, they: (1) can be effective in deterring systematic or persistent exploitation of these disposal sites; and (2) can reduce the likelihood of inadvertent, intermittent human intrusion to a degree to be determined by the implementing agency. However, the Agency believes that passive institutional controls can never be assumed to eliminate the chance of inadvertent and intermittent human intrusion into these disposal sites (EPA, 1985, p. 38088c).

Wherever human intrusion is mentioned in the standard and in the Supplementary Information to the Standard, the references are to inadvertent human intrusion. Statements such as the following suggest that the requirement for passive, institutional controls is to protect against inadvertent human intrusion:

The most speculative potential disruptions of a mined geologic repository are those associated with inadvertent human intrusion. ...The Agency believes that the most productive consideration of inadvertent human intrusion concerns those realistic possibilities that may be usefully mitigated by repository design, site selection, or use of passive controls (although passive institutional controls should not be assumed to completely rule out the possibility of intrusion). Therefore, inadvertent and intermittent intrusion by exploratory drilling for resources (other than provided by the disposal system itself) can be the most severe intrusion scenario assumed by the implementing agencies (EPA, 1985, p. 38088c-38089a).

The following statement suggests that once the warning message has been correctly communicated, a potential intruder will cease activity in the area:

Furthermore, the implementing agencies can assume that passive institutional controls or the intruders' own exploratory procedures are adequate for the intruders to soon detect, or be warned of, the incompatibility of the area with their activities (EPA, 1985, p. 38089a,b).

\subsection{Background}

\subsubsection{Future Societies}

The effort undertaken by the Performance Assessment (PA) Department at SNL to design markers for the WIPP builds upon the work of an earlier effort that identified the range of possible future societies that may occur in the vicinity of the WIPP during the next 10,000 years (Hora et al., 1991). The possible modes of humans intruding into a repository, specifically, the WIPP, 
and the probabilities of such intrusions were considered in this earlier study.

Before one can communicate with future societies about the location and dangers of the wastes, it is important to consider with whom one is trying to communicate. The question of future societies was addressed using a multidisciplinary panel of experts in fields deemed pertinent. This group was called the Futures Panel, and included individuals with backgrounds in history, future studies, economics, law, physics, sociology, geography, engineering, political science, risk analysis, agriculture, climatology, history, and demographics.

The panel was organized into four teams, and each team was given the same charge in order to facilitate a focused but diverse set of responses. The teams were named based on the predominant geographical location of the members: Boston Team, Southwest Team, Washington A Team, and Washington B Team. In addition to the panel members being given a specific task, they were trained in providing judgments in a numerical fashion and provided with background information about the WIPP Project (Weart et al., 1991).

Each team of the Futures Panel analyzed the question of future societies differently. The reports describing the analysis of the problem, prepared by each team, were reproduced in Hora et al. (1991). Hora et al. (1991) also provide a full discussion of the possible future societies, modes of intrusions, and probabilities of intrusions elicited from the teams. The material in the individual team reports expanded the view of what future societies might be like. Not all of the modes of intrusion considered by the teams would be inadvertent. The focus of marking the WIPP is to communicate what is buried in the repository and the possible consequences of intruding into the repository. The applicable regulation (discussed in the previous section) states that it is most important to communicate to protect against inadvertent human intrusion and states the assumption that once a potential intruder realizes the location and dangers of the waste buried in the repository, such activity will cease. Some of the modes of intrusion postulated by the Futures Panel are beyond what is currently required by the applicable regulations for analysis of the future performance of the WIPP.

The Boston Team developed several underlying factors that were believed to impact future societal activities and possible modes and frequencies of intrusion. Certain time periods after the end of the expected 100 years of active institutional control after closure (100-300 years, 300-3,000 years, or 3,000-10,000 years after closure) and possible levels of technology (lower, similar to today, or higher) were considered to impact all of the possible modes of intrusion. Knowledge of the past, the value of the materials, the level of industrial activity, and population density are the 
other factors that are important in influencing human actions and the extent of human intrusion. The possible modes of intrusion developed by the Boston Team are resource exploration and extraction, reopening the WIPP for additional storage, waste disposal by injection wells, archaeological exploration, explosive testing, and water impoundment. After the first 300 years after closure, the Boston Team did not believe that boreholes would be drilled in the WIPP area for resource exploration and extraction because of total removal and/or the use of nonpetroleum energy sources.

The Southwest Team based its outlook on the possible intrusion into the WIPP by future societies in political control of the area around the WIPP (the United States of America or another political entity) and the technological development pattern (steady increase from today's level, steady decline from today's level, or a fluctuating seesaw pattern). Possible modes of intrusion associated with a steady increase in the level of technology are deep strip mining and exotic mining techniques that could develop in the future. Conventional drilling and excavation activities were associated with a steady decline in the level of technology or a seesaw situation. The Southwest Team did not make a distinction in their analysis for time periods, stating that society could cycle through the three technological development patterns throughout the 10,000 years.

The Washington A Team examined conditions today in terms of technology level and both energy and other natural-resource use and developed possible futures by extrapolating these factors. The possible futures thus developed are continuity (a continuation of current trends), radical increase (large growth in the use of resources), discontinuity (fluctuations in levels of technology and resource use), and steady state (emphasizing renewable resources and compatibility with the earth). Time was another factor with both the period of 0-200 years and 200-10,000 years after closure of the WIPP being considered. Exploration for and development of resources were considered the most likely modes of intrusion. Other modes included construction between cities of a deep tunnel that would intersect the WIPP, water impoundment, development of well fields, and explosions.

The Washington B Team based its examination of possible future societies and modes of intrusion on the underlying factors of the level of wealth and technology, government control (prudent and effective in controlling the area of the WIPP, or not), climate (relating to water supply development), and the price of resources (more than doubling current levels, or not). The Washington $B$ Team considered the two time periods of 0-200 years and 200-10,000 years after closure of the WIPP, as the near and far futures, respectively. The activities future societies might be undertaking that were believed to be able to cause intrusion of the repository were resource exploration and extraction, development of water wells, scientific 
investigations, and weather modification. The far future for resource exploration and extraction only extends from 200-500 years after closure. After that time, all the oil and gas would have been removed and/or society would no longer be on a petroleum-based economy.

\subsubsection{Marker Development}

The Markers Panel was charged with developing design guidelines for markers to be placed at the WIPP and with developing preliminary forms of messages and formats to communicate the location and dangers of the wastes buried there, for the regulatory period of 10,000 yęars. The charge was to consider both individual components and an entire marker system. After a marker-system design was developed based on the guidelines, the panelists were asked to estimate the probability over time that the marker system would continue to exist and that the messages would be interpretable. The estimation of probabilities (function of time, technology, and mode of intrusion) is discussed in Chapter 5.

The nature of the design-criteria problem imposed a number of constraints on the work of the Markers Panel. The Futures Panel input suggested that societies quite different from our own may be controlling and inhabiting the area of the WIPP. The markers must be developed to communicate with people whose culture may not be directly descended from our own. This possible cultural change is in addition to the changes in language that normally occur over time, even when societies are in continuous contact. Secondly, the period of regulatory concern $(10,000$ years $)$ requires that the marker materials, construction techniques, and placement be able to withstand the forces of nature and the tendency of human beings to vandalize structures or to remove pieces. Thirdly, the markers must be able to convey complex information, not just about wastes hidden from view, but also about the hazards of radioactivity as a function of time.

The Markers Panel addressed the complexities of the task by relying on the strengths of a multidisciplinary panel. The individuals on the panel represent disciplines pertinent to addressing the materials and communications aspects of the marker issue. Thus, geomorphology, materials science, and engineering were included to address the issues of markers withstanding natural and human-induced degradation and destructive forces. Design and architecture addressed the design and placement of structures. Archaeology provided information about the materials and structural configurations that historically have been successful in remaining intact over long periods of time. Through the study of human social and cultural development, anthropology brought to the marker effort the understanding of how humans process information and communicate. Linguistics was important to 
the development of the messages in view of how languages and meanings have evolved through time and the necessity of using linguistic messages that can easily be decoded. Semiotics addressed communication not only with languages, but with signs and symbols. Previous efforts to think broadly about communication in terms of using radio signals or sending a satellite into space to communicate over long time periods with unknown beings led to the selection of individuals from the astronomy and communications disciplines for the Panel. The broad educational backgrounds and work experience of the panelists (related to the various technical aspects of this question) meant that there was broad discussion and cooperation (people not limited to their own specific field) in the development of the design criteria.

\subsection{Purposes of the Study}

This study had two purposes, one qualitative and one quantitative. These purposes were instituted in response to the requirements and guidance of the Standard. The qualitative purpose was developing design guidelines for markers and messages to communicate with future societies about the location and danger of the buried wastes at the WIPP. Such information is intended to deter inadvertent human intrusion. The results of the Markers Panel will be considered in developing the final design and in constructing the markers. The quantitative purpose was to estimate the efficacy of the markers in surviving the required time period and in communicating the intended messages. Other passive institutional controls (such as a records system or a protective barrier system) need to be developed and could also be effective in deterring inadvertent human intrusion. Consideration of other passive controls and their effectiveness in deterring intrusion was beyond the scope of this task. 


\section{ORGANIZATION AND METHODOLOGY}

\subsection{Using Expert Judgment}

The methodology employed in this study to obtain quantitative evaluations of the proposed marker systems performance is referred to as expert-judgment analysis (Bonano et al., 1990). For some aspects of performance assessment for radioactive waste repositories, it is not possible to build models, conduct experiments, or make observations to resolve uncertainties. While certain aspects of marker design such as material decay and symbol recognition can be studied for short periods of time, it is not possible to assess the performance of such a system entirely using these traditional data sources. When unresolvable uncertainties do exist, expert judgments are often used to quantify the uncertainties and to express both the known and the unknown.

The formalization of expert-judgment elicitation for nuclear waste repositories is described in Bonano et al. (1990). Expert judgment is pervasive in complex analyses. Judgments about the selection of models, experimental conditions, and data sources must be made. The choice is not whether expert judgment will be used; instead, the choice is whether it will be collected and used in a disciplined, explicit manner or utilized implicitly where its role in the analysis is not obvious.

Precursor studies have provided a structure for the collection of expert judgment. These studies include, among others, the Electric Power Research Institute (EPRI, 1986) study of seismicity in the eastern United States, the NUREG-1150 study (U.S. NRC, 1990), and the recently completed study of futures of society (Hora et al., 1991). These studies provide models for the collection of expert judgments. These models are designed to avoid the pitfalls that interfere with the collection process.

A formal expert-judgment process should consist of several well-defined activities. Such activities include creating issue statements for the experts to respond to, selecting experts and training them in probability assessment, eliciting probabilities and other information, and processing and presenting findings.

While the NUREG-1150 study was most central in the design of this current effort, there are substantial differences between them that are important to note. The goal of the expert-judgment process in NUREG-1150 was to provide uncertainty distributions for parameters and to judge the likelihood of specific phenomena. The uncertain quantities were relatively well defined. In the present study of marker systems, the issues are less well defined, and 
the experts are required to employ substantial creative effort in devising marker systems and evaluating their potential performance.

Several organizational forms for experts in an elicitation process have been described (Bonano et al., 1990). One form is the organization of experts into teams. A team structure is useful when disparate disciplines need to be used on a given problem. An added benefit of using teams is enhanced communication among the experts. In contrast, when experts from different disciplines work on separate, but connected, parts of the same problem, coordination and communication among the experts must be explicitly provided.

Through the work that was done with the Futures Panel and the Markers Panel, PA has developed its own procedure for the use of expert judgment. This procedure is documented by Rechard et al., 1992.

\subsection{Expert-Judgment Panel}

\subsubsection{Decision to Use an Expert-Judgment Panel}

The decision to use the expert judgment process to develop information on markers was based both on the importance of the topic and the lack of alternate sources of this information. Human intrusion appears to be the only credible means by which radionuclides may reach the accessible environment (Marietta et al., 1989; Guzowski, 1990). Deterring human intrusion through the use of markers could significantly enhance confidence in compliance with the standard. The handling of such a sensitive topic must be done in an open and documented format allowing input from individuals outside of the WIPP Project. In addition, the design of markers is interdisciplinary and must utilize input from many disciplines. Further, estimation of the efficacy of markers in deterring human intrusion cannot be done any other way than through expert judgment--experiments cannot provide this type of information.

\subsubsection{Development of the Issue Statement}

The development of the issue statement is the first step in the process of conducting an expert judgment panel. Development of the issue statement is important not only to clearly define the issue to be addressed by the panel, but also as a means of identifying the disciplines that need to be represented on the panel. 
The issue statement for the Markers Panel is found in Appendix A. It requires judgments for both marker and message design characteristics and estimates of performance of the marker system. Performance of the markers was to be estimated for both the "physical" longevity of the markers and the ability of the markers to convey the correct message to deter inadvertent human intrusion. Marker-design characteristics include a general description of the marker system, as well as a physical description of each marker component within the marker system, including size, location, shape, and materials. Also, the teams were asked to describe the messages upon or within the markers and the method(s) of conveying the messages. For performance of the system of markers, the teams were asked to assess the extent to which the marker system they designed would survive, be correctly interpreted, and evoke the correct response over the 10,000 year period of regulatory concern. The estimates of performance were requested for the individual marker components as well as for the entire system.

The issue statement in Appendix $\mathrm{A}$ is the version provided to the Markers Panel. This issue statement was changed once the Markers Panel began their work. Such modifications are not inappropriate if the experts believe that certain questions cannot be answered or the problem should be examined another way. As a result of the emphasis on inadvertent human intrusion, as discussed in Chapter 1 , the panel members did not provide probabilities that the correctly interpreted messages would be heeded (i.e., probabilities were not provided for question 6). Team A stated that

The regulatory requirement is to deter inadvertent human intrusion, and thus we feel that if the message is understood, our job is completed. Any action that takes place after the message is understood is advertent and intentional.

Team B stated that

We cannot guarantee that any simple or complex message, even when recognized and correctly interpreted, will deter a human being from inappropriate action.... Nevertheless, carefully designed warnings could be expected to reduce the chances of inadvertent intrusion into the WIPP. Moreover, an intrusion would not be casual, but would be a planned event. As such, there would be a greater likelihood to consider cautionary data.

A further change was made to the issue statement. Both teams stated that they had developed system designs and that it was inappropriate to consider the effectiveness of individual marker components. 


\subsubsection{Selection of Experts}

Marker design depends upon the expertise of many disciplines, so a multidisciplinary team approach was needed. The disciplines expected to be important included anthropology, archaeology, architecture, astronomy, communications, design, engineering, geology/geophysics, modern languages, linguistics, materials science, psychology, semiotics, and sociology. In addition, parallel teams were to be established to elicit diversity in the responses. Because the teams were to be composed of scientists and scholars from many disciplines, the pool of candidates needed to be sufficiently broad. An established nomination process was employed to achieve this.

\subsubsection{NOMINATION PROCESS}

The selection of experts begins with the identification of persons believed sufficiently knowledgeable in the disciplines identified by SNL staff as being pertinent to the project to nominate experts. The nominators were identified through contacts with professional organizations, such as the American Institute of Professional Geologists, the Linguistic Society of America, and the American Anthropological Association. Governmental organizations such as the U.S. Soil Conservation Service and the National Climatic Data Center were also contacted, as were public interest organizations such as the League of Women Voters. Simultaneously, literature searches were performed in the publications of the above listed disciplines. From these literature searches, prominent authors were identified and contacted. The editors of professional journals were also contacted concerning nominations.

An initial contact was usually made by telephone to explain the project to the potential nominator. This contact was used to determine whether the potential nominator would be able to provide nominations and to assist in obtaining the cooperation of other people in the project.

The identification of nominators and the initial contacts took place between June 13 and July 13, 1990. By July 24, 1990, a formal request for nominations (Appendix B) had been sent to all nominators who had agreed to contribute. This letter outlined the tasks to be accomplished by the experts, provided a tentative schedule, and included a description of the criteria to be used for selection of experts. The letter invited selfnomination if the nominator deemed this to be appropriate.

During the following week, additional letters were sent to those nominators who had not responded to the request for nominations. Several potential nominators, who were thought to be sufficiently knowledgeable or 
their responses considered to be highly desirable but could not be contacted verbally, were also sent letters. The parties to whom these letters were addressed are shown in Appendix $C$.

From this effort, a total of 92 nominations were obtained by August 8, 1990. By August 14, 1990, a letter was sent to each of the nominees (Appendix D). This letter outlined the tasks to be accomplished and firm dates for the two meetings to be held in Albuquerque. The nominees, if interested and able to participate in the project, were asked to send a letter describing their interests and any special qualifications relevant to the WIPP marker-development study. A curriculum vitae was also requested from each nominee. Letters of interest and curriculum vitae were received from 57 nominees by noon of August 20, 1990. After that time, no further responses were considered.

\subsubsection{SELECTION ADVISORY COMMITTEE}

The selection advisory committee assisted the PA Department by evaluating the interest letter and the curriculum vitae from all of the nominees in light of the selection criteria and by making recommendations for the membership of the Markers Panel as well as several alternates. The selection advisory committee was composed of three university professors with some knowledge of the WIPP Project and the expert judgment process: Dr. G. Ross Heath of the University of Washington (oceanography), Dr. Douglas G. Brookins of the University of New Mexico (geology), and Dr. Detlof von Winterfeldt of the University of Southern California (decision analysis). Dr. Heath is also the chair of the WIPP Performance Assessment Peer Review Panel, which gave him special insights into the project-related goals of the WIPP PA Project and the regulatory framework of the Project.

The members of the selection committee were provided with copies of the above information several days prior to the meeting during which the final recommendations were made. The recommendations of the selection advisory committee were followed in establishing the Markers Panel.

Criteria for the selection of experts were drafted for use by the selection advisory committee. These criteria were similar to the criteria that were distributed to the nominators and nominees but also included criteria related to the balance of disciplines and geographic location of the teams. The criteria are included in this report as Appendix E. 


\subsubsection{SELECTION OF PANEL}

The selection advisory committee recommended members for two teams within the Markers Panel, and these recommendations were accepted in establishing the Markers Panel. The Markers Panel consisted of one team of six members and one team of seven members. Two teams with parallel missions provided a focused but diverse set of responses. The size of the teams was dictated, in part, on the necessity of representing the pertinent disciplines. Table 2-1 lists the members of the Markers Panel, their affiliations, and their discipline (s).

\subsubsection{Panel Deliberations}

The Markers Panel first met as a group November 4-6, 1991, in Albuquerque, New Mexico. The first meeting included presentations regarding the WIPP Project, the Standard, WIPP performance assessment, and the issue statement (the specific questions the teams were asked to address), as well as long-term climate variability at the WIPP, and the geologic and hydrologic characteristics of the WIPP region as they relate to marker development. At this meeting, the panel members also received an introduction to the expert judgment process and training in the process of expert judgment elicitation. On November 5, the Markers Panel toured the WIPP surface facilities, underground facilities, and surrounding area. Originally, the Markers Panel was scheduled to convene in October 1990, to coincide with the meeting at which the Futures Panel discussed their results. The convening of the Markers Panel was postponed for one year because of budgetary constraints. In order to make the connection between the work of the Futures and Markers Panels, each member of the Markers Panel was provided with the reports prepared by the four Futures Panel teams and text of the background information provided to the Futures Panel. In addition, one person from each of the four Futures Panel teams attended the November meeting to discuss their team's results and to answer questions.

The Markers Panel was also provided with literature related both to the WIPP Project and human intrusion, as well as other efforts to address deterring human intrusion into nuclear waste repositories.

After the first meeting when the members of the two teams began developing a strategy for addressing the issue statement, each team met separately for working sessions. (Team A met December 5-6, 1991, in Buffalo, New York, and Team B met December 14-16, 1991, in Kona, Hawaii.)

The two Markers. Panel teams presented their results and draft reports to SNL staff, federal and state agency representatives, Nuclear Energy Agency 
(NEA) Human Intrusion Working Group observers, and several members of the press January 13-14, 1992, in Albuquerque, New Mexico.

Table 2-1. Marker Development Panel

\begin{tabular}{|c|c|c|}
\hline Team/Names & Organization(s) & Discipline(s) \\
\hline \multicolumn{3}{|l|}{$\underline{A}$} \\
\hline Ast, Dieter & Cornell University & Materials Science \\
\hline Brill, Michael & $\begin{array}{l}\text { Buffalo Organization for } \\
\text { Social and Technological } \\
\text { Innovation }\end{array}$ & $\begin{array}{l}\text { Architecture, } \\
\text { Environmental Design }\end{array}$ \\
\hline Goodenough, Ward & University of Pennsylvania & $\begin{array}{l}\text { Anthropology, } \\
\text { Linguistics }\end{array}$ \\
\hline Kaplan, Maureen & Eastern Research Group & $\begin{array}{l}\text { Archaeology, } \\
\text { Environmental Engineering }\end{array}$ \\
\hline Newmeyer, Frederick & University of Washington & Linguistics \\
\hline Sullivan, Woodruff & University of Washington & $\begin{array}{l}\text { Astronomy, } \\
\text { Communications }\end{array}$ \\
\hline \multicolumn{3}{|l|}{$\underline{B}$} \\
\hline Baker, Victor & University of Arizona & Geomorphology \\
\hline Drake, Frank & $\begin{array}{l}\text { University of California } \\
\text { at Santa Cruz }\end{array}$ & $\begin{array}{l}\text { Astronomy } \\
\text { Communications }\end{array}$ \\
\hline Finney, Ben & $\begin{array}{l}\text { University of Hawaii at } \\
\text { Manoa }\end{array}$ & Anthropology \\
\hline Givens, David & $\begin{array}{l}\text { American Anthropological } \\
\text { Association }\end{array}$ & Anthropology \\
\hline Lomberg, Jon & Consultant & Scientific Illustration \\
\hline Narens, Louis & $\begin{array}{l}\text { University of California } \\
\text { at Irvine }\end{array}$ & Semiotics \\
\hline Williams, Wendell & $\begin{array}{l}\text { Case Western Reserve } \\
\text { University }\end{array}$ & Materials Science \\
\hline
\end{tabular}




\section{RECOMMENDED DESIGN CHARACTERISTICS}

Team A and Team B of the Markers Panel were both given the same issue statement (the same set of questions) to address during their deliberations. The issue statement contained a number of requirements and constraints within which the Panel needed to work. The time frame for the Panel to consider must be 10,000 years because of the requirement that performance assessments cover a time period of 10,000 years after closure of the disposal facility (Containment Requirements). The second requirement was that the markers must be developed with a goal of being able to convey information to any future society (considering the broad spectrum of possible future societies developed by the Futures Panel [Hora et al., 1991]).. The third requirement was to communicate the dangers associated with the waste buried at the WIPP.

A comparitson of the two sets of marker design characteristics highlights the aspects of marker design where the two teams are in agreement. A comparison of the approaches also allows one to see the diversity in the responses and highlights those competing approaches to markers that need to be investigated further.

The reader is directed to Appendices $F$ and $G$ for the Team $A$ and Team $B$ reports, respectively. The reports are reproduced as received by the project coordinator except for (1) correcting typographical errors, (2) editing for internal format consistency, (3) renumbering, repositioning, and captioning figures, (4) updating the tables of contents to be in line with the previous changes, and (5) changing the text in accordance with answers to a number of questions that were addressed to the individual teams about their reports as written. The members of the Markers Panel reviewed a draft copy of this report and the updated versions of Appendices $F$ and $G$, and responded to the questions provided. The Team A report contains a number of marker alternatives that were considered and rejected by the team and are included in order to show the range of the thought process. The Team A final recommendation is for the use of the "Menacing Earthworks" along with the other components discussed below and in their report. The Team B report is a discussion of their recommended marker system.

This report uses a number of terms that need to be clarified. A marker system is the entire set of physical structures (whatever their form or composition) emplaced to communicate to future societies about the wastes buried in the repository. If earthen berms and buried message disks are used to mark a repository, their combination would constitute a system. The earthen berms and the message disks each would be considered components of the marker system. Each individual message disk would be a marker element. 


\subsection{Team A}

\subsubsection{Basic Premises}

Team A listed their goal in communication as the simultaneous fulfillment of three objectives: (1) to provide a gestalt message (the whole message is greater than the sum of the parts/components), (2) to use a systems approach, and (3) to incorporate redundancy in the markers.

For the gestalt message, the purpose is to convey a message not just with words and pictures, but through the very vehicles of conveying the messages, and the messages themselves. That is, the marker materials, their construction, and their arrangement are such that future generations coming upon the markers will understand the message that this place is not one where people would want to spend a lot of time. With the gestalt message, the emphasis is on communicating through the entire marker system.

The systems approach to designing and constructing markers is that the various marker components are linked to each other and supplement the information (or fill in any gaps) from other marker components. Messages are provided in different levels of complexity, in different formats, and convey different aspects of the entire message.

The redundancy within the marker components provides enough individual markers of any one type (material or message or arrangement) so that if some are vandalized or degraded over time, there are sufficient numbers remaining to communicate the required message. The size and construction of the markers can also provide redundancy in that the form of the communication is overdone so that it can still communicate after degradation or defacement. With earthen berms (discussed later in this. section), the size called for would allow the marker to withstand considerable erosion and still remain recognizable as a human construction marking an area.

\subsubsection{Assumptions/Bases}

Team A made the following assumptions that impacted their marker designs and their recommendations for future studies. While various civilizations have developed and declined over time, history has shown that since literacy first developed 6000 years ago, it has not ceased to exist (Appendix $F$, Section 1.2). Team A assumed that scholarship capable of translating the messages on the markers will continue to exist somewhere in the world during the time period being considered. This resulted in a major emphasis on written language, and the redundancy of the written languages to aid in decipherment. 
Team A assumed that, based on past history, political boundaries are impermanent, and so included the importance of an international effort that would maintain knowledge of the location of all nuclear waste disposal sites.

The evolution of existing cultures and the creation of new ones over the next 10,000 years cannot be known. Thus, a marking system and the messages must be cross-cultural to the extent possible. The marking system must be rooted in basic human concepts and understanding.

\subsubsection{Message Levels and Media}

Team A recommended the use of five levels of messages in the overall marker system. These five levels are a modification of the following four levels defined by Givens (1982; also see Appendix F, P. F-34), who is also a member of Team B:

Level I: Rudimentary Information: "Something manmade is here,"

Leve1 II: Cautionary Information: "Something manmade is here and it is dangerous,"

Level III: Basic Information: Tells what, why, when, where, who, and how (in terms of information relay, not how the site was constructed), and

Level IV: Complex Information: Highly detailed, written records, tables, figures, graphs, maps, and diagrams.

With the gestalt message, the marker system itself would be able to communicate both Level I and Level II information. Team A created a new Level IV with the level of complexity of information to be between those of the Level III and Level IV messages defined by Givens (1982). The most complex information, Level $\mathrm{V}$, would be the "complete rulemaking record" and would be stored in archives.

In an effort to achieve the three objectives in Section 3.1.1, the team set out to be as unambiguous as possible in how the warning messages were conveyed. This led to a greater reliance on communicating through a sense of place, through written languages and scientific symbols for the specific information, and through the use of the human face with expressions.

Communicating through a sense of place is based on the concept of human archetypes--that all human beings react similarly to particular physical 
environments. The team believed that creating an environment that communicated to humans today that the area around the markers was not a welcoming one, would also communicate the same message to future human beings, at least within the time frame required in the standard.

Language was seen as an unambiguous means of communicating specific information about the repository, as were scientific media such as the periodic table of the elements and star charts. The recommended languages are those of the United Nations (Arabic, English, Spanish, French, Russian, and Chinese) and that of the largest group of Native Americans in the area (Navajo). Space should also be left on the markers for a future society to add a language to the markers. The periodic table of the elements is distinctive in shape and should be recognizable. Drawing on humans' traditional observation of the stars, a chart could be developed to show the positions of the stars when the WIPP was closed and after 10,000 years.

Human facial expressions were seen as unambiguous because humans use the same expressions to convey particular feelings, independent of culture. There is less emphasis on what were perceived as potentially ambiguous pictographs. Team members thought that while human figures and animals would be recognized in the future, the intent of the messages might be lost. For example, one can recognize people and animals in ancient cave drawings but not know what the artists were trying to communicate about them.

\subsubsection{Marker System Components}

The individual components that comprise the marker system developed by Team A vary with regard to size, materials, specific message and audience, and location. The system can best be explained by discussing it in the sequence of marker components that would be encountered as someone approached the outside and moved to the center. Team $A$ has stated that certain specific aspects of the design require testing before being finalized.

The area over the waste panels (and a buffer area to account for migration of the radioactive materials) would be outlined by earthen berms (Appendix F, Figs. 4.3-8 and 4.3-9). These berms would be jagged in shape and would radiate out from, but not cover, a central, generally square area. The number of berms is sufficient to delineate a central area or "keep" even if some are destroyed. The four corner berms would be higher and provide a "vantage point" to see the area as a whole. The jagged nature of the berms is meant to convey a sense of foreboding (not honorific or pleasant). The exact size, shape, and configuration of the berms would be such that they would not quickly be eroded or covered. The earthworks are meant to convey a Level I message. 
Within the "keep" would be multiple "message kiosks" (Appendix F, Fig. 4.3-18) containing Level II messages in approximately seven languages (those of the United Nations plus a local indigenous language), as well as Level III messages in several of the languages plus a local indigenous language. Space will be left on the kiosk for a future generation to inscribe the message in another language. The construction of the kiosk will include a concrete "mother" wall that will be built to curve around and protect an inner granite wall containing the actual messages. Messages will be placed high up on the wall so as not to be buried by blowing sand and to make it more difficult for individuals to deface them.

The Level IV information, the most complex at the site, will be contained in concrete rooms (Appendix F, Fig. 4.3-17). One such room will be buried in each of the four corner berms, allowing them to be exposed as the berms erode. The rooms will be constructed to allow access but to prevent the removal of informational materials. The "sliding stone entry plug" will protect an opening large enough for a human to enter and leave, but too small to allow removal of an intact stone slab containing the information. Level IV information will be located on stone slabs on the interior walls. Two additional layers of stone slabs with the same messages will be located behind the original layer in case the original wall is damaged or destroyed. In addition, each Level IV room will contain other types of information such a periodic table of the elements to indicate what is buried at depth, and an astronomical calendar to indicate at what point in the past the wastes were buried.

From the top of the earthworks, one would be able to see a world map showing other disposal sites (Appendix F, Fig. 4.3-16), as well as part of the original buildings left as a message center ("left to decay"). The location of the WIPP on the world map will be indicated by a marker that will also sit atop a Level IV room beneath the map.

\subsubsection{Other Design Requirements}

Team A made a number of recommendations about the design and construction of markers to increase the probability that they will remain recognizable far into the future. Irregularly shaped "blocks" to be used for construction (e.g., message rooms) would make recycling of the blocks for the construction of other structures more difficult. The individual marker elements (e.g., message kiosks) should be large enough to make them difficult to carry off to a future museum. Materials for the construction of the marker elements (message kiosks, message chambers, world map) should have the lowest intrinsic value feasible so that their materials are not worth removing and recycling. 


\subsection{Team B}

\subsubsection{Basic Premises}

Team B developed a list of 10 items that guided their work in developing a marker system. These items relate to the rationale and moral aspects, how to mark, and future activities. Their design was guided by the need for durability of markers and clarity of messages. The team report addresses markers by examining possible alternatives in terms of persistence of markers, recognition of markers and messages, interpretation of the messages, and deterrence of human intrusion. A discussion of each of the 10 items follows.

(1) Two of the four teams that comprised the Futures Panel (Hora et al., 1991) recommended to the Markers Panel that the site not be marked so as not to draw curious visitors to the WIPP. Team B disagreed and stated that because of current mining and petroleum production in the area, the site must be marked to reduce the probability of inadvertent human intrusion.

(2) The marker strategy must not rely on one location for message carriers, but should use both surface and buried markers. Surface markers would be available for interpretation now and in the future. Buried markers could become available to communicate in the future through possible erosion if the surface markers have been removed, destroyed, or degraded through natural processes. Buried markers could also reinforce the message of surface markers during possible intrusion attempts. If humans begin to intrude upon the site, buried markers (safe from vandals and certain natural weathering processes) could communicate the dangers below. The buried markers also reinforce the message if the surface markers are misinterpreted or ignored.

(3) The messages must be truthful. All people have the right to know the potential impacts of their actions. In addition, if future people discover that part of a message is untrue, they may not believe any of the message.

(4) The outer extent of the marker system should be visible from the center. This allows a visitor (if they are in the center of the marker system) to cognitively assemble all the markers they are seeing as delineating a coherent site or message about this particular location.

(5) The area to be marked should be that area above the waste panels. Part of the reason for this delineation is found in (3). If a large area is marked to communicate that one should not dig or drill here because of the hazardous material buried below, and if future societies drill within the designated area but outside the area of the panels and find nothing unusual, 
they may not believe the other messages. The second reason is found in (4). It may be difficult to convey a sense of a coherent marker system that is attempting to communicate over, for example, what is believed will be the controlled area (16 square miles). People may not be able to relate a marker at one point to something that is two miles away, because of the limits of human perception.

(6) The highest probability of success in correctly communicating the location and nature of the buried wastes is to repeat the message in a number of ways so that if one message form is not completely understood, the message in another form may fill in the gaps and reinforce it. The linguistic material must use simple sentences so that future scholars will be more readily able to translate it. The different modes of communication must communicate with different societies having knowledge of or access to different levels of technology. This duplication is necessary because we cannot know what cultures will be like or what levels of technology will be in existence at any future time. The team noted that the message from the Futures Panel (Hora et al., 1991) was that the Markers Panel should make recommendations for a wide variety of cultures and technologies.

(7) While current plans call for removing the existing buildings, parking lots, roads, etc. and returning the area to its previous condition, Team $B$ recommended that part of the main building containing the "hot cell" should be left in place for the benefit of future archaeologists--to study it and understand what took place at the WIPP.

(8) Detailed information about the WIPP should be stored off-site, but the details of what information should be stored and where and how it should be stored, should be developed in the future, closer to the time when such a record system would be implemented.

(9) The marking of nuclear-waste repositories should have an international aspect in terms of a map at the site showing other disposal sites around the world to ensure that all knowledge is not lost. This marking may also include either the existing radiation trefoil symbol or a symbol still to be developed.

(10) Testing of markers and messages must be undertaken between now and the time of implementation. This will include testing both for durability (materials and inscriptions) and cross-cultural understanding of the messages. 


\subsubsection{Assumptions/Bases}

Team $B$ was directed in their actions by the recommendations of the Futures Panel. In developing markers, Team B believed that a systems approach (many types of markers, messages, and communication modes) would be the most useful in communicating under the unknown and varying circumstances of what the Washington A Team of the Futures Panel called "radical discontinuity." Under radical discontinuity, society would have gone through considerable changes-political, social, and technological--that might impact existing knowledge bases, languages, and institutional controls and memory. Messages would thus need to communicate to everyone regardless of their culture, technology, or political structure, that not intruding upon the repository was in their own best interest. A second assumption made by the team was that political change will take place (i.e., resulting in the United States of America not being in control of the area around the repository). This assumption led Team $B$ to be concerned with making the marking of repositories an international effort. A third assumption made by Team $B$ was that vandalism will continue to be a tendency of some parts of human society. Multiple marker elements of one component (i.e., the placement of many stone monoliths in the marker system) will allow for the marker component to remain and be able to be interpreted even if some of the individual elements are destroyed or removed.

\subsubsection{Message Levels and Media}

Team B recommended the presentation of messages in four levels based on the work of Givens (1982; also see Appendix G, PP. G-17 and G-36):

Level I: Rudimentary Information. The site itself and its component parts would announce "Something made by humans is here." The most important property of a Level-I sign is its own existence. "Human made" would be suggested by the patterned shape--the unnatural syntax and negative entropy--of the earthwork, rock structures and inscriptions.

Level II: Cautionary Information. Elementary linguistic scripts and pictographic narratives would convey: "Warning, dangerous materials are buried below."

Level III: Basic Information. Level III messages, including longer linguistic narratives, pictographic sequences, maps and simple diagrams would explain basic what, why, when, where, who and how information about the site. 
Level IV: Complex Information. Highly detailed written records, scientific data and diagrams would be available at the site in inscriptions and buried "time capsules."

Team B has delineated the ways in which messages about the WIPP should be conveyed to future societies. The first message medium is through written language. The languages used for these messages would be the main written languages in use today (such as English, Spanish, German, Russian, Japanese, and Chinese), liturgical languages (such as Latin, Hebrew, and Arabic), and the languages of the Native Americans in the area (such as Navajo, Hopi, and Mescalero Apache). Language would be expected to communicate both the basic and complex information about the WIPP. Scientific diagrams would be used to communicate some of the more complex information about the elements buried at the WIPP (the periodic table), the elapsed time since the WIPP was closed (a diagram showing the 26,000-year precession of the stars in the sky), or the stratigraphy of the area (a model that uses samples of materials from the formations between the surface and the repository arranged in the proper order and scale to indicate what would be encountered during a potential intrusion). Pictographs would be used to communicate information about how the WIPP was constructed, how far underground the waste is buried, the activities that should not be undertaken in the area, and what might happen if the waste is disturbed. Some sort of radioactive symbol might be used in text and on the marker elements to make the connection between radioactivity and what is buried in the repository.

\subsubsection{Marker System Components}

The marker-system components recommended by Team B will be discussed in the sequence they would be encountered by a visitor approaching the area. Team B believed that by the mere existence of a marker system and by observing the effort that went into creating it, a future society would realize that this was something important (markers are there for a purpose) and worth saving. The largest, outermost component, the berms (earthworks), are encountered first (Appendix G, Figs. 1 and 2). The berms define the marked area above the waste panels, but do not completely cover the area above the waste panels. If an international symbol has been developed by the time the marker system is implemented, the berms could be in that shape. To last for the 10,000-year period of regulatory concern, the berms must be massive (to withstand human and natural forces), on the order of 30 -ft-high, constructed of local earth and caliche. The berms would be spiked with materials with properties anomalous to the naturally occurring ones (e.g., "different dielectric, radar reflective, and magnetic properties") for detection by aircraft or satellite equipment. Because the berms outline the area above the waste panels, the hot cell of the WIPP buildings, which Team B recommended be left in place, is located outside the berms. 
Within the outline of the berms, granite monoliths (specific number to be a power of two for easier reconstruction) would be erected in a circular pattern. They would be large in size to withstand natural erosion and to deter the removal by humans. The monoliths themselves would be of two types: taller, narrower ones (25-ft-high by 10 -ft-wide) designed not to be buried by blowing, accumulating sand; and shorter, wider ones (10-ft-high by 20-ftwide) "difficult to topple or decapitate." Even the accumulation of sand around monoliths will still mark the area. The monoliths would be inscribed on "protected surfaces" (physically protected from erosion by sand and/or water) with warning messages in the languages discussed previously. Inscribed monoliths also would be buried within the earthworks for future discovery, and granite plugs would be placed in one or more of the shafts originally leading to the repository level and in off-site archives. The importance of placing markers in the shafts is based on the belief that future societies would be able to determine where the shafts were located because of anomalies in the materials and/or densities of the shaft materials.

Also salted in the earthworks and in the area within the earthworks would be "time capsules" (6-in. to 2 -ft in diameter) buried deep enough not to be discovered initially by souvenir hunters; the capsules would be placed to be found by those beginning to intrude upon the site--e.g., by archaeologists - or as the earthworks erode. These "time capsules" (clay, ceramics, glass, or sintered alumina) would have information inscribed on the outside. Samples of wood might be included to allow a future society to date the marker activities through carbon-14 dating.

In the center of the marker system would be a granite structure (20-ft by $30-\mathrm{ft}$ ) containing the most complex information about the time of the placement of the waste, location, and dangers of the waste. This information (conveyed through the use of language, pictographs [Appendix G, Figs. 5 through 15], and diagrams) would be inscribed on protected, flat exposed surfaces of the structure. Specific examples include a world map of all known nuclear waste sites at the time of marker emplacement, the periodic table of the elements indicating the radioactive elements contained in the repository, and a diagram showing the precessional cycle of the earth in relation to the time of burial and the time of the reading (Appendix G, Figs. 15 and 16). In addition, models containing samples of the various layers of materials that would be encountered while drilling through the material overlying the waste panels, including the relative location of the shafts and waste panels, would be available both at the site and in other locations (Appendix G, Fig. 4). 


\section{PRINCIPLES OF MARKING}

The purposes of marking a nuclear-waste disposal site are to inform future generations of the site's location and to warn of the hazards associated with the nuclear waste buried at the location. To achieve these purposes, certain principles should be followed in the design of the markers and the development of the messages to maximize the time over -which the markers will physically survive with the messages intact and to maximize the interpretability of the messages in view of the potential variety of cultural changes that can occur. The subject areas where these principles need to be identified are architecture, linguistics, material properties, and message levels, and were drawn from the design characteristics developed by Team A and Team B. The goals and principles of each subject area are described in this chapter. Table 4-1 is a summary of these design principles.

\subsection{Architectural-Design Principles}

The principles that need to be included in the design of markers depend on the goals of the markers. These goals are the definition of an area that future generations should avoid disturbing and the definition of this area extending for as reasonably far into the future as possible given the resource limitations of any disposal program.

A single monument defines a spot and is therefore not an adequate approach to marking a disposal location. In order to define an area that future generations should avoid, a single, large marker covering the area of concern or a system of individual monuments or elements of a marker in a pattern surrounding the area should be used. Either marker size, monument, or marker-element pattern can convey to future generations that the structure is not a natural feature. When using a system of marker elements, the sense of an area can be conveyed by a design of structural continuity (e.g., other parts of the marker system or component can be seen from any other location or marker element). Continuity of design allows the recognition of patterns in the marker component(s) or element(s) even with part of the component or element removed, destroyed, or damaged.

To assure longevity, several principles should be used to guide the design of the markers and/or monuments. The design should assure structural stability and durability. Structural stability refers to the marker component or element being able to withstand natural processes and events and retain the original orientation and position. Examples of the types of potential disruptions are winds associated with intense storms and seismic ground motion caused by earthquakes. Stability can be enhanced by designing the components and elements to be massive with low centers of gravity or to 


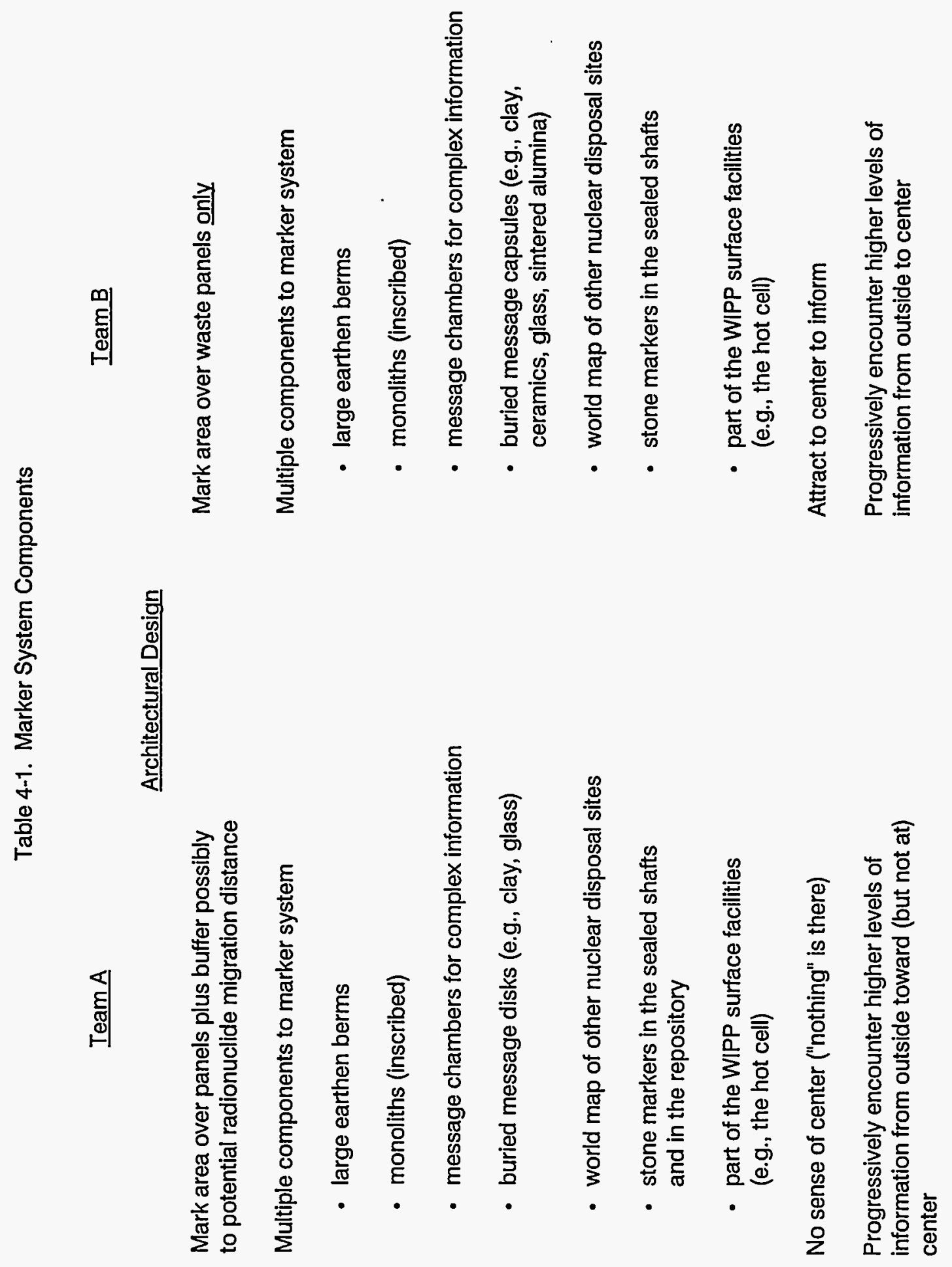


Table 4-1. Marker System Components (continued)

Team A

\section{Linguistics}

Less emphasis on pictographs

Use human facial expressions (horror and sickness)

Use several languages

Greater reliance on sense of place

Reliance on language

Accurately convey the risk of intrusion (not an attempt to scare)

Material Properties

Use low-value materials

- local materials for berms

- "common" rock for monoliths (e.g., granite)

\section{Message Levels}

5 levels of messages

Levels based on complexity of message

\section{Team B}

Prominent role for pictographs

Use several languages

Purely functional area; not artistic

Reliance on language

Accurately convey the risk of intrusion (not an attempt to scare)

Use low-value materials

- local materials for berms

- "common" rocks for monoliths (e.g., granite) 
$\underline{T e a m}$ A

\section{$\underline{\text { Team B }}$}

\section{Other Message Media}

Star map and celestial marker to indicate time since closure

Placement near the surface of materials with magnetic and electrical conductivity properties anomalous to those of the naturally occurring materials in the area

\section{Periodic table of the elements}

\section{Aeolian structures}

Models of the WIPP's surface facilities, stratigraphy, shafts, and waste panels

International radiation symbol used with text and other media

\section{Other Marking Components}

\section{Public information effort (current)}

International standard for the basic design features for long-term marking

Testing for the longevity of markers and the interpretability of messages across cultures

Off-site archives
Star map and celestial marker to indicate time since closure

Placement near the surface of materials with magnetic and electrical conductivity properties anomalous to those of the naturally occurring materials in the area

Periodic table of the elements and diagrams of nuclear reactions

Models of the WIPP's surface facilities, stratigraphy, shafts, and waste panels

International biohazard symbol used with text and other media

International standard for the basic design features for long-term marking

Testing for the longevity of markers and the intepretability of messages across cultures

Off-site archives (including duplicates of markers) 
be physically anchored to the ground. Durability generally is dependent on the material properties of the markers, although durability can be enhanced by design. For example, aerodynamic design can be used to mitigate the effects of wind-blown sand abrasion.

\subsection{Linguistic Principles}

The current structure of society undoubtedly will undergo changes over time, and these changes may be either gradual or abrupt, continuous or discontinuous. Changes in society can include governmental and economic structures, cultural values, religion, language, and level of technology. The linguistic goal of the marker system is to transmit a warning to future societies about the hazards posed by the buried nuclear waste at a particular disposal location regardless of these societal changes. Several principles should be applied to the development of this warning.

Because languages evolve over time and can be replaced by "new" languages, the warning message should be kept simple for each level of societal development being targeted for contact. This simplicity should be applied to the message itself (e.g., be direct and not misleading), the content of the message (e.g., eliminate extraneous information), and the grammatical structure within the message (e.g., avoid complex sentences and colloquialisms).

Another principle to employ is redundancy. Different cultures may have differing capabilities for interpreting messages and the format in which the message is presented. To account for such differences in capability, redundancy should be incorporated into the message through the use of language, symbols, and graphics as deemed appropriate.

Even without major changes in interpretive abilities, cultural and political changes may occur that can be countered through language redundancy. For those portions of the message conveyed by language, the use of more than one language may increase the likelihood that future societies will understand the message.

\subsection{Material-Properties Principles}

The material properties of the markers are of critical importance to the goal of marking a disposal location for an extended period of time. This time period is a significant portion of the time period of regulatory concern limited by the constraint of resource allocation within the overall program relative to the hazard posed by the waste being disposed of. Under ideal 
conditions, the markers should be designed to survive for the entire time period of regulatory concern. Material properties play a major role in determining the physical survivability of the markers in the natural environment. These properties also can affect the type and longevity of the messages being transmitted over this time period.

Principles that determine the suitability of material properties of the markers focus on the topics of durability, reactivity, and desirability. Durability refers to the ability of a material to withstand both current and projected climatic conditions. Weather-related processes include but are not limited to wetting and drying, freezing and thawing, and thermal expansion and contraction, along with wind-blown sand abrasion. Materials exposed to these processes should not suffer significant degradation during extended periods of exposure. Material properties also are important in resisting the effects of both individual and societal vandalism.

The materials used for the markers should be nonreactive (inert) for the time frame being considered, the environmental conditions expected, and geologic setting at the disposal location. Reactivity refers to the chemical interaction between two or more materials in contact with one another. The reactivity concern is both between materials used to construct marker elements and between the markers and the local geologic material upon which the marker rests or is embedded or buried. With naturally occurring materials, the chemistry may change as climatic conditions change. For example, a wetter climate may result in changes in vegetative population, which in turn affect the chemistry of soils being developed. Interaction between the soils and the marker material could affect the longevity of the marker.

Another factor that will play a major role in the longevity of the markers is the desirability of the marker material(s) for use by future societies. The material properties of the marker material(s) should be selected to minimize the potential resource value for reprocessing or recycling.

\subsection{Message-Level Principles}

As was the case for linguistics, future societal changes also are likely to affect the type of message that can be interpreted. Scientifically and technologically advanced societies may be more inquisitive than substantially less developed societies and require more information to satisfy their curiosity. For one type of society, a simple warning of danger may be sufficient to deter intrusion, whereas another society may require an explanation of why the area is dangerous before intrusion is deterred. 
Because of the possible diversity of future societies and their differing abilities to decipher messages and their differing incentives for heeding the messages, more than one message level should be used to convey the warning about a waste-disposal location. The contents of these messages should be based on the principles of redundancy and complexity.

Redundancy assures that each message level conveys a similar warning about the potential hazards of the location. Level of complexity targets variously scientifically and technologically developed societies based on their estimated ability to decipher a message. Whereas linguistic redundancy repeats the same specific message at a particular level of complexity in different languages, message-level redundancy repeats the same basic message at different degrees of complexity. The number of message levels and the degree of message complexity in each level depends on the spectrum of development of future societies that are expected to pose an intrusion threat to the disposal facility. 


\section{PROBABILITY ELICITATION}

The elicitation of probabilities of the efficacy of proposed marker designs was accomplished in, formal sessions during which the experts were assisted in representing their beliefs as probabilities. The sessions took place in Albuquerque, New Mexico, January 13 and 14, 1992. The probability elicitation sessions were held on the second day of a two day meeting. During the first day, the teams made presentations of design characteristics and discussed marker systems. This agenda allowed the sharing of information and ideas between the expert teams.

On the second day of the meeting, each team worked with a normative specialist, an individual familiar with decision analysis, to encode judgment probabilities. Professor Ravinder (University of New Mexico) and Timothy Wheeler (SNL, Dept. 6641) were the normative specialists for this study, for Team $B$ and Team A respectively, and worked under the direction of Professor Stephen Hora (University of Hawaii). In each session, a member of the WIPP Performance Assessment staff (Kathleen Trauth [6342] for Team A and Robert Guzowski [Science Applications International Corporation] for Team B) was present to assist by clarifying issues as required.

Members of the Nuclear Energy Agency Working Group on Human Intrusion, who were meeting simultaneously in Albuquerque, attended both the first day's presentations and the elicitation session for Team A.

The teams were asked to consider two questions: (1) durability of the marker system and (2) interpretability of the marker system. The marker system used as a basis for making judgments was the marker system presented the day before by the respective team. Although the original intent of the elicitation session was to obtain probabilistic assessments for each component in the marker system, the complexity and interdependency among the components of the system thwarted this goal.

\subsection{Persistence of Markers}

Markers Team A members addressed the probabilities of markers continuing to exist on an individual basis so that six individual assessments were given. Assessments were provided assuming three different levels of societal technology--high, medium or current day, and low at five points in time--200, $500,1,000,5,000$, and 10,000 years after closure. Table 5-1 contains the probabilities of the marker system (as defined in the report by Team A) continuing to exist at the given epoch, conditional on a dominant state of technology. 
Table 5-1. Probabilities of the Marker System Persisting - Team A

\begin{tabular}{|c|c|c|c|c|c|c|}
\hline \multirow[b]{2}{*}{ Expert } & \multirow{2}{*}{$\begin{array}{l}\text { Dominant } \\
\text { Technology }\end{array}$} & \multicolumn{5}{|c|}{ Years After Closure } \\
\hline & & 200 & 500 & 1,000 & 5,000 & 10,000 \\
\hline Ast & $\begin{array}{l}\text { High } \\
\text { Medium } \\
\text { Low }\end{array}$ & $\begin{array}{l}.99 \\
.99 \\
.99\end{array}$ & $\begin{array}{l}.98 \\
.98 \\
.98\end{array}$ & $\begin{array}{l}.95 \\
.95 \\
.95\end{array}$ & $\begin{array}{l}.75 \\
.75 \\
.75\end{array}$ & $\begin{array}{l}.50 \\
.60 \\
.60\end{array}$ \\
\hline Brill & $\begin{array}{l}\text { High } \\
\text { Medium } \\
\text { Low }\end{array}$ & $\begin{array}{l}.99 \\
.99 \\
.99\end{array}$ & $\begin{array}{l}.98 \\
.98 \\
.98\end{array}$ & $\begin{array}{l}.95 \\
.95 \\
.95\end{array}$ & $\begin{array}{l}.70 \\
.70 \\
.85\end{array}$ & $\begin{array}{l}.50 \\
.50 \\
.80\end{array}$ \\
\hline Goodenough & $\begin{array}{l}\text { High } \\
\text { Medium } \\
\text { Low }\end{array}$ & $\begin{array}{l}.99 \\
.99 \\
.99\end{array}$ & $\begin{array}{l}.98 \\
.98 \\
.98\end{array}$ & $\begin{array}{l}.90 \\
.95 \\
.98\end{array}$ & $\begin{array}{l}.85 \\
.90 \\
.95\end{array}$ & $\begin{array}{l}.70 \\
.75 \\
.80\end{array}$ \\
\hline Kaplan & $\begin{array}{l}\text { High } \\
\text { Medium } \\
\text { Low }\end{array}$ & $\begin{array}{l}.95-.99 \\
.95-.99 \\
.95-.99\end{array}$ & $\begin{array}{l}.95-.99 \\
.95-.99 \\
.95-.99\end{array}$ & $\begin{array}{l}.90-.95 \\
.90-.95 \\
.90-.95\end{array}$ & $\begin{array}{l}.80 \\
.80 \\
.90\end{array}$ & $\begin{array}{l}.70 \\
.70 \\
.85\end{array}$ \\
\hline Newmeyer & $\begin{array}{l}\text { High } \\
\text { Medium } \\
\text { Low }\end{array}$ & $\begin{array}{l}.90 \\
.95 \\
.95\end{array}$ & $\begin{array}{l}.85 \\
.90 \\
.90\end{array}$ & $\begin{array}{l}.70 \\
.85 \\
.85\end{array}$ & $\begin{array}{l}.65 \\
.80 \\
.85\end{array}$ & $\begin{array}{l}.60 \\
.60 \\
.65\end{array}$ \\
\hline Sullivan & $\begin{array}{l}\text { High } \\
\text { Medium } \\
\text { Low }\end{array}$ & $\begin{array}{l}.90 \\
.95 \\
.95\end{array}$ & $\begin{array}{l}.85 \\
.90 \\
.90\end{array}$ & $\begin{array}{l}.80 \\
.85 \\
.85\end{array}$ & $\begin{array}{l}.70 \\
.80 \\
.80\end{array}$ & $\begin{array}{l}.50 \\
.70 \\
.70\end{array}$ \\
\hline
\end{tabular}

During the probability assessments, the members of Team A made the following observations:

At some point in the future, a high technology society may be able to remove the entire WIPP or may decide to remove the markers. During the early time periods, the distinction between the levels of technology is not as great as during later periods simply because the differences have not had time to develop.

In contrast, Team B provided consensus probabilities at three points in time--500, 2,000, and 10,000 years after closure. Table 5-2 contains these consensus probabilities for the three levels of technology.

\subsection{Interpretability of Messages}

The second question addressed by the teams of experts is whether, given that the markers are extant, the message will be interpreted correctly by the potential intruders. This question was asked conditionally for several time 
Table 5-2. Consensus Probabilities of the Marker System Persisting - Team B

\begin{tabular}{|c|c|c|c|}
\hline \multirow{2}{*}{$\begin{array}{l}\text { Dominant } \\
\text { Technology }\end{array}$} & \multicolumn{3}{|c|}{ Years After Closure } \\
\hline & 500 & 2,000 & 10,000 \\
\hline $\begin{array}{l}\text { High } \\
\text { Medium } \\
\text { Low }\end{array}$ & $\begin{array}{l}.90 \\
.90 \\
.90\end{array}$ & $\begin{array}{l}.85 \\
.80 \\
.70\end{array}$ & $\begin{array}{l}.85 \\
.60 \\
.40\end{array}$ \\
\hline
\end{tabular}

periods, for the three levels of technology (higher than current levels, at current levels, or lower than current levels), and for six modes of intrusion--drilling for water, mineral exploration, drilling to create injection wells, archaeological investigation, and other scientific investigation.

Because of the motivations for potential intrusions and the individuals expected to be involved, both Team A and Team B tended to group archaeology and scientific exploration together, and to group together mineral exploration, and drilling wells for water supply or waste disposal. In general (across time periods and levels of technology), individuals involved in potential intrusions for archaeological and other scientific purposes were estimated as having greater likelihoods of correctly interpreting the warning information at the WIPP than those individuals involved in mineral exploration or drilling wells for water supply or waste disposal. Archaeologists and other scientists might be expected to have access to local, regional, and international information sources that could provide additional information about the WIPP. Within the Team A judgments, distinctions were sometimes made among mineral exploration, drilling wells for water supply, and drilling wells for waste disposal because of judgments about whether the activities were local efforts or represented a large societal effort. Individuals involved with those activities believed to require a larger, more organized effort were judged to have a higher probability of correctly interpreting the messages because of the greater access to information.

The probability that the marker system will deter the potential intruders has been assessed as a function of time, the state of technology and the mode of intrusion. Tables 5-3 through 5-7 give the probability of correct interpretation for each of the five modes of intrusion. The first six lines in each table give the correct interpretation probability for the experts of Team A while the seventh line is the consensus probability for Team $B$. 
Table 5-3. Probability of Correct Interpretation of Message--Drilling for Water as Mode of Intrusion

\begin{tabular}{|c|c|c|c|c|c|c|c|c|c|c|c|c|c|c|c|}
\hline \multirow[b]{2}{*}{ Expert } & \multicolumn{3}{|c|}{$\begin{array}{c}200 \text { Years } \\
\text { Technology = }\end{array}$} & \multicolumn{3}{|c|}{$\begin{array}{c}500 \text { Years } \\
\text { Technology }=\end{array}$} & \multicolumn{3}{|c|}{$\begin{array}{c}1,000 \text { Years } \\
\text { Technology }=\end{array}$} & \multicolumn{3}{|c|}{$\begin{array}{c}5,000 \text { Years } \\
\text { Technology }=\end{array}$} & \multicolumn{3}{|c|}{$\begin{array}{l}10,000 \text { Years } \\
\text { Technology }=\end{array}$} \\
\hline & $\overline{H^{1}}$ & $M$ & $\bar{L}$ & $\mathrm{H}$ & $M$ & $L$ & $\mathrm{H}$ & $M$ & $L$ & $\mathrm{H}$ & $\bar{M}$ & $L$ & $\bar{H}$ & M & $L$ \\
\hline $\begin{array}{l}\text { Ast } \\
\text { Brill } \\
\text { Goodenough } \\
\text { Kaplan } \\
\text { Newmeyer } \\
\text { Sullivan }\end{array}$ & $\begin{array}{l}.99 \\
.99 \\
.99 \\
.99 \\
.99 \\
.95\end{array}$ & $\begin{array}{l}.98 \\
.99 \\
.99 \\
.98 \\
.99 \\
.95\end{array}$ & $\begin{array}{l}.98 \\
.95 \\
.99 \\
.95 \\
.90 \\
.80\end{array}$ & $\begin{array}{l}.98 \\
.95 \\
.95 \\
.98 \\
.90 \\
.90\end{array}$ & $\begin{array}{l}.95 \\
.95 \\
.95 \\
.90 \\
.85 \\
.90\end{array}$ & $\begin{array}{l}.60 \\
.90 \\
.70 \\
.70 \\
.80 \\
.60\end{array}$ & $\begin{array}{l}.95 \\
.95 \\
.90 \\
.95 \\
.80 \\
.85\end{array}$ & $\begin{array}{l}.85 \\
.95 \\
.90 \\
.85 \\
.70 \\
.85\end{array}$ & $\begin{array}{l}.20 \\
.70 \\
.50 \\
.60 \\
.50 \\
.40\end{array}$ & $\begin{array}{l}.90 \\
.95 \\
.65 \\
.80 \\
.70 \\
.70\end{array}$ & $\begin{array}{l}.10 \\
.95 \\
.60 \\
.70 \\
.60 \\
.70\end{array}$ & $\begin{array}{l}.05 \\
.60 \\
.15 \\
.40 \\
.40 \\
.10\end{array}$ & $\begin{array}{l}.90 \\
.95 \\
.50 \\
.75 \\
.50 \\
.40\end{array}$ & $\begin{array}{l}.05 \\
.95 \\
.40 \\
.50 \\
.30 \\
.40\end{array}$ & $\begin{array}{l}.01 \\
.50 \\
.02 \\
.01 \\
.20 \\
.01\end{array}$ \\
\hline Sullivan & \multicolumn{3}{|c|}{500 Years } & \multicolumn{3}{|c|}{2000 Years } & \multicolumn{3}{|c|}{10,000 Years } & & & & & & \\
\hline Team B & .90 & .90 & .80 & .90 & .85 & .70 & .99 & .80 & .30 & & & & & & \\
\hline
\end{tabular}


Table 5-4. Probability of Correct Interpretation of Message--Mineral Exploration as Mode of Intrusion

\begin{tabular}{|c|c|c|c|c|c|c|c|c|c|c|c|c|c|c|c|}
\hline \multirow[b]{2}{*}{ Expert } & \multicolumn{3}{|c|}{$\begin{array}{c}200 \text { Years } \\
\text { Technology }=\end{array}$} & \multicolumn{3}{|c|}{$\begin{array}{c}500 \text { Years } \\
\text { Technology }=\end{array}$} & \multicolumn{3}{|c|}{$\begin{array}{c}1,000 \text { Years } \\
\text { Technology }=\end{array}$} & \multicolumn{3}{|c|}{$\begin{array}{c}\text { 5,000 Years } \\
\text { Technology }=\end{array}$} & \multicolumn{3}{|c|}{$\begin{array}{l}10,000 \text { Years } \\
\text { Technology }=\end{array}$} \\
\hline & $\overline{\mathrm{H}^{1}}$ & $M$ & $\bar{L}$ & $\mathrm{H}$ & $M$ & $L$ & $\mathrm{H}$ & $M$ & $\bar{L}$ & $\mathrm{H}$ & $M$ & $\bar{L}$ & $\mathrm{H}$ & $M$ & $L$ \\
\hline $\begin{array}{l}\text { Ast } \\
\text { Brill } \\
\text { Goodenough } \\
\text { Kaplan } \\
\text { Newmeyer } \\
\text { Sullivan }\end{array}$ & $\begin{array}{l}.99 \\
.99 \\
.99 \\
.99 \\
.99 \\
.95\end{array}$ & $\begin{array}{l}.99 \\
.99 \\
.99 \\
.98 \\
.99 \\
.95\end{array}$ & $\begin{array}{l}.98 \\
.95 \\
.99 \\
.95 \\
.90 \\
.80\end{array}$ & $\begin{array}{l}.98 \\
.95 \\
.95 \\
.98 \\
.90 \\
.90\end{array}$ & $\begin{array}{l}.95 \\
.95 \\
.95 \\
.90 \\
.85 \\
.90\end{array}$ & $\begin{array}{l}.70 \\
.90 \\
.70 \\
.70 \\
.80 \\
.60\end{array}$ & $\begin{array}{l}.95 \\
.95 \\
.90 \\
.97 \\
.80 \\
.85\end{array}$ & $\begin{array}{l}.90 \\
.95 \\
.90 \\
.85 \\
.70 \\
.85\end{array}$ & $\begin{array}{l}.50 \\
.70 \\
.50 \\
.65 \\
.50 \\
.40\end{array}$ & $\begin{array}{l}.90 \\
.95 \\
.65 \\
.95 \\
.70 \\
.70\end{array}$ & $\begin{array}{l}.20 \\
.95 \\
.60 \\
.80 \\
.60 \\
.70\end{array}$ & $\begin{array}{l}.10 \\
.60 \\
.15 \\
.50 \\
.40 \\
.10\end{array}$ & $\begin{array}{l}.90 \\
.95 \\
.50 \\
.90 \\
.50 \\
.40\end{array}$ & $\begin{array}{l}.20 \\
.95 \\
.40 \\
.75 \\
.30 \\
.40\end{array}$ & $\begin{array}{l}.05 \\
.50 \\
.02 \\
.02 \\
.20 \\
.01\end{array}$ \\
\hline Sullivan & \multicolumn{3}{|c|}{500 Years } & \multicolumn{3}{|c|}{2000 Years } & \multicolumn{3}{|c|}{10,000 Years } & & & & & & \\
\hline Team B & .90 & .90 & .80 & .90 & .85 & .70 & .99 & .80 & .30 & & & & & & \\
\hline
\end{tabular}

1The levels of technology being more advanced than today $(H)$, similar to today's level $(M)$, and less advanced than today $(L)$. 
Table 5-5. Probability of Correct Interpretation of Message--Drilling for Disposal Wells as Mode of Intrusion

\begin{tabular}{|c|c|c|c|c|c|c|c|c|c|c|c|c|c|c|c|}
\hline \multirow[b]{2}{*}{ Expert } & \multicolumn{3}{|c|}{$\begin{array}{c}200 \text { Years } \\
\text { Technology }=\end{array}$} & \multicolumn{3}{|c|}{$\begin{array}{c}500 \text { Years } \\
\text { Technology }=\end{array}$} & \multicolumn{3}{|c|}{$\begin{array}{c}\text { 1,000 Years } \\
\text { Technology = }\end{array}$} & \multicolumn{3}{|c|}{$\begin{array}{c}5,000 \text { Years } \\
\text { Technology }=\end{array}$} & \multicolumn{3}{|c|}{$\begin{array}{l}10,000 \text { Years } \\
\text { Technology }=\end{array}$} \\
\hline & $\mathrm{H}^{1}$ & $M$ & $L$ & $\vec{H}$ & $M$ & $L$ & $\mathrm{H}$ & $M$ & $\mathrm{~L}$ & $\mathrm{H}$ & $\bar{M}$ & $\bar{L}$ & $\mathrm{H}$ & $M$ & $L$ \\
\hline $\begin{array}{l}\text { Ast } \\
\text { Brill } \\
\text { Goodenough } \\
\text { Kaplan } \\
\text { Newmeyer } \\
\text { Sullivan }\end{array}$ & $\begin{array}{l}.99 \\
.99 \\
.99 \\
.99 \\
.99 \\
.95\end{array}$ & $\begin{array}{l}.98 \\
.99 \\
.99 \\
.98 \\
.99 \\
.95\end{array}$ & $\begin{array}{l}.98 \\
.95 \\
.99 \\
.95 \\
.90 \\
.80\end{array}$ & $\begin{array}{l}.98 \\
.95 \\
.95 \\
.98 \\
.90 \\
.90\end{array}$ & $\begin{array}{l}.95 \\
.95 \\
.95 \\
.90 \\
.85 \\
.90\end{array}$ & $\begin{array}{l}.60 \\
.90 \\
.70 \\
.70 \\
.80 \\
.60\end{array}$ & $\begin{array}{l}.95 \\
.95 \\
.90 \\
.97 \\
.80 \\
.85\end{array}$ & $\begin{array}{l}.85 \\
.95 \\
.90 \\
.85 \\
.70 \\
.85\end{array}$ & $\begin{array}{l}.20 \\
.70 \\
.50 \\
.65 \\
.50 \\
.40\end{array}$ & $\begin{array}{l}.90 \\
.95 \\
.65 \\
.95 \\
.70 \\
.70\end{array}$ & $\begin{array}{l}.10 \\
.95 \\
.60 \\
.80 \\
.60 \\
.70\end{array}$ & $\begin{array}{l}.05 \\
.60 \\
.15 \\
.50 \\
.40 \\
.10\end{array}$ & $\begin{array}{l}.90 \\
.95 \\
.50 \\
.90 \\
.50 \\
.40\end{array}$ & $\begin{array}{l}.05 \\
.95 \\
.40 \\
.75 \\
.30 \\
.40\end{array}$ & $\begin{array}{l}.01 \\
.50 \\
.02 \\
.02 \\
.20 \\
.01\end{array}$ \\
\hline Sullivan & \multicolumn{3}{|c|}{500 Years } & \multicolumn{3}{|c|}{2,000 Years } & \multicolumn{3}{|c|}{10,000 Years } & & & & & & \\
\hline Team B & .90 & .90 & .80 & .90 & .85 & .70 & .99 & .80 & .30 & & & & & & \\
\hline
\end{tabular}


Table 5-6. Probability of Correct Interpretation of Message--Archaeological Investigation as Mode of Intrusion

\begin{tabular}{|c|c|c|c|c|c|c|c|c|c|c|c|c|c|c|c|}
\hline \multirow[b]{2}{*}{ Expert } & \multicolumn{3}{|c|}{$\begin{array}{c}200 \text { Years } \\
\text { Technology }=\end{array}$} & \multicolumn{3}{|c|}{$\begin{array}{c}500 \text { Years } \\
\text { Technology }=\end{array}$} & \multicolumn{3}{|c|}{$\begin{array}{c}1,000 \text { Years } \\
\text { Technology }=\end{array}$} & \multicolumn{3}{|c|}{$\begin{array}{c}5,000 \text { Years } \\
\text { Technology }=\end{array}$} & \multicolumn{3}{|c|}{$\begin{array}{l}10,000 \text { Years } \\
\text { Technology }=\end{array}$} \\
\hline & $\mathrm{H}^{1}$ & $\bar{M}$ & $L$ & $\mathrm{H}$ & $M$ & $L$ & $\mathrm{H}$ & $M$ & $\mathrm{~L}$ & $\mathrm{H}$ & $M$ & $L$ & $H$ & $M$ & $L$ \\
\hline $\begin{array}{l}\text { Ast } \\
\text { Brill } \\
\text { Goodenough } \\
\text { Kaplan } \\
\text { Newmeyer } \\
\text { Sullivan }\end{array}$ & $\begin{array}{l}.9999 \\
.999 \\
.99 \\
.99 \\
.99 \\
.99\end{array}$ & $\begin{array}{l}.9999 \\
.999 \\
.99 \\
.99 \\
.99 \\
.99\end{array}$ & $\begin{array}{l}.99 \\
.99 \\
.95 \\
.99 \\
.90 \\
.90\end{array}$ & $\begin{array}{l}.9999 \\
.999 \\
.99 \\
.99 \\
.95 \\
.97\end{array}$ & $\begin{array}{l}.9999 \\
.99 \\
.99 \\
.99 \\
.90 \\
.97\end{array}$ & $\begin{array}{l}.98 \\
.95 \\
.80 \\
.95 \\
.75 \\
.70\end{array}$ & $\begin{array}{l}.999 \\
.99 \\
.98 \\
.95 \\
.85 \\
.90\end{array}$ & $\begin{array}{l}.98 \\
.97 \\
.98 \\
.95 \\
.85 \\
.90\end{array}$ & $\begin{array}{l}.95 \\
.87 \\
.60 \\
.70 \\
.40 \\
.60\end{array}$ & $\begin{array}{l}.98 \\
.99 \\
.90 \\
.95 \\
.70 \\
.80\end{array}$ & $\begin{array}{l}.95 \\
.96 \\
.90 \\
.90 \\
.60 \\
.80\end{array}$ & $\begin{array}{l}.50 \\
.75 \\
.40 \\
.60 \\
.20 \\
.20\end{array}$ & $\begin{array}{l}.90 \\
.99 \\
.90 \\
.90 \\
.60 \\
.60\end{array}$ & $\begin{array}{l}.70 \\
.95 \\
.80 \\
.75 \\
.20 \\
.60\end{array}$ & $\begin{array}{l}.40 \\
.60 \\
.20 \\
.10 \\
.10 \\
.03\end{array}$ \\
\hline Sullivan & \multicolumn{3}{|c|}{500 Years } & \multicolumn{3}{|c|}{ 2,000 Years } & \multicolumn{3}{|c|}{10,000 Years } & & & & & & \\
\hline Team B & .99 & .99 & .90 & .99 & .95 & .85 & .99 & .90 & .45 & & & & & & \\
\hline
\end{tabular}


- Table 5-7. Probability of Correct Interpretation of Message--Scientific Investigation as Mode of Intrusion

\begin{tabular}{|c|c|c|c|c|c|c|c|c|c|c|c|c|c|c|c|}
\hline \multirow[b]{2}{*}{ Expert } & \multicolumn{3}{|c|}{$\begin{array}{c}200 \text { Years } \\
\text { Technology }=\end{array}$} & \multicolumn{3}{|c|}{$\begin{array}{c}500 \text { Years } \\
\text { Technology }=\end{array}$} & \multicolumn{3}{|c|}{$\begin{array}{c}1,000 \text { Years } \\
\text { Technology }=\end{array}$} & \multicolumn{3}{|c|}{$\begin{array}{c}5,000 \text { Years } \\
\text { Technology }=\end{array}$} & \multicolumn{3}{|c|}{$\begin{array}{c}10,000 \text { Years } \\
\text { Technology }=\end{array}$} \\
\hline & $\mathrm{H}^{1}$ & $M$ & $L$ & $\bar{H}$ & $M$ & $\mathrm{~L}$ & $\mathrm{H}$ & $M$ & $\bar{L}$ & $\mathrm{H}$ & $M$ & $\bar{L}$ & $\bar{H}$ & $\bar{M}$ & $L$ \\
\hline $\begin{array}{l}\text { Ast } \\
\text { Brill } \\
\text { Goodenough } \\
\text { Kaplan } \\
\text { Newmeyer } \\
\text { Sullivan }\end{array}$ & $\begin{array}{l}.9999 \\
.999 \\
.99 \\
.99 \\
.99 \\
.99\end{array}$ & $\begin{array}{l}.9999 \\
.999 \\
.99 \\
.99 \\
.99 \\
.99\end{array}$ & $\begin{array}{l}.99 \\
.99 \\
.95 \\
.99 \\
.90 \\
.90\end{array}$ & $\begin{array}{l}.9999 \\
.999 \\
.99 \\
.99 \\
.95 \\
.97\end{array}$ & $\begin{array}{l}.9999 \\
.99 \\
.99 \\
.99 \\
.90 \\
.97\end{array}$ & $\begin{array}{l}.98 \\
.95 \\
.70 \\
.95 \\
.75 \\
.70\end{array}$ & $\begin{array}{l}.999 \\
.99 \\
.98 \\
.95 \\
.85 \\
.90\end{array}$ & $\begin{array}{l}.98 \\
.97 \\
.98 \\
.95 \\
.85 \\
.90\end{array}$ & $\begin{array}{l}.95 \\
.87 \\
.60 \\
.70 \\
.40 \\
.60\end{array}$ & $\begin{array}{l}.98 \\
.99 \\
.90 \\
.90 \\
.70 \\
.80\end{array}$ & $\begin{array}{l}.95 \\
.96 \\
.90 \\
.85 \\
.60 \\
.80\end{array}$ & $\begin{array}{l}.50 \\
.75 \\
.30 \\
.50 \\
.20 \\
.20\end{array}$ & $\begin{array}{l}.90 \\
.99 \\
.90 \\
.75 \\
.60 \\
.60\end{array}$ & $\begin{array}{l}.70 \\
.95 \\
.80 \\
.50 \\
.20 \\
.60\end{array}$ & $\begin{array}{l}.40 \\
.60 \\
.10 \\
.05 \\
.10 \\
.03\end{array}$ \\
\hline Sullivan & \multicolumn{3}{|c|}{500 Years } & \multicolumn{3}{|c|}{2,000 Years } & \multicolumn{3}{|c|}{10,000 Years } & & & & & & \\
\hline Team B & .99 & .99 & .85 & .99 & .95 & .80 & .99 & .90 & .45 & & & & & & \\
\hline
\end{tabular}




\subsection{Conclusions and Implementation}

As the teams worked to develop a system of markers for the WIPP, they identified a number of fundamental principles that guided their work and that should guide future marker panel development efforts. These fundamental principles began with the moral imperative to mark the WIPP (in agreement with the mandated use of markers at a disposal site in 40 GFR Part 191, Subpart B) and to be truthful in the messages rather than attempting to frighten or mislead future societies. The teams also identified the need for multiple levels of messages (corresponding to the complexity of the information) on multiple types of markers, the importance of linking the markers to off-site archives, and the necessity of using materials of little intrinsic value that would be difficult to recycle.

The two teams agreed and disagreed in different aspects of marker-system design and thus produced the desired diversity in potential designs. - Both teams recommended the use of earthen berms, stone markers, small buried message markers, message chambers, and markers connected to outside archives in their designs. The disagreement between the teams centered on whether to attempt to use the principle of human archetypes in communicating through the marker system (communicating through the feeling evoked by the markers) or whether to develop a marker system that communicates purely through the construction and arrangement of the markers and the messages on the markers.

All the probability sets show a high probability ( $85 \%$ or greater) that markers will persist in a recognizable form for 500 years after closure of the WIPP, with many of the estimates in the 95-99\% range. With time, the estimates of marker persistence decrease for all three levels of the dominant technology. By 10,000 years, estimates of marker persistence range from 408 probability to $85 \%$ probability, with most of the estimates in the $60-70 \%$ range.

The probability of correct interpretation varies with time and with the mode of intrusion, with high probabilities (90-998) in the earlier (up to 500) years and for high technology. By 10,000 years, the probabilities of correct interpretation have decreased, particularly for a society with a low level of technology.

The high probabilities of both persistence and interpretability in the first 500 years after closure of the WIPP would provide the greatest protection during the period of continued petroleum exploration and extraction. As stated in Chapter 1 , the Boston Team and the Washington $B$ Team of the Futures Panel believed that resource exploration and extraction in the WIPP area would cease within 300-500 years after closure. 
The estimates of marker persistence and interpretability from the Markers Panel will be used with the estimates of intrusion rates for various modes of intrusion from the Futures Panel to provide input on inadvertent human intrusion for performance assessments performed by the WIPP PA Department.

The estimates provided by the panel members show their belief that a marker system can be designed and constructed to persist and to communicate the location and dangers of the wastes buried in the repository far into the 10,000 year period of regulatory concern. Further study in some of the areas outlined in this report will be necessary prior to the final design and construction of the marker system. These topics include (1) physical properties--durability of marker materials under current conditions at the WIPP, mechanism of attaching or inscribing messages, and the interaction of wind/sand/water with marker materials and configurations; (2) interpretation of graphic or pictorial messages that are independent of culture; and (3) interpretation of written messages that are independent of culture. The implementation of the test results and the Panel recommendations in the actual design and construction of the marker system will ensure that the system is as durable as possible and as effective as possible in communicating the appropriate messages. 


\section{REFERENCES}

Bertram-Howery, S.G., and R.L. Hunter. 1989. Plans for Evaluation of the Waste Isolation Pilot Plant's Compliance with EPA Standards for Radioactive Waste Management and Disposal. SAND88-2871. Albuquerque, NM: Sandia National Laboratories.

Bonano, E.J., S.C. Hora, R.L. Keeney, and D. von Winterfeldt. 1990. Elicitation and Use of Expert Judgment in Performance Assessment for HighLevel Radioactive Waste Repositories. NUREG/CR-5411, SAND89-1821. Albuquerque, NM: Sandia National Laboratories.

EPRI (Electric Power Research Institute). 1986. Seismic Hazard Methodology for the Central and Eastern United States. Volume 1, Part 2: Methodology. EPRI NP-4726. Palo Alto, CA: Electric Power Research Institute.

Givens, D.B. 1982. "From Here to Eternity: Communicating with the Distant Future," Et cetera. Vol. 39, no. 2, 159-179.

Guzowski, R.V. 1990. Preliminary Identification of Scenarios That May Affect the Escape and Transport of Radionuclides From the Waste Isolation Pilot Plant, Southeastern New Mexico. SAND89-7149. Albuquerque, NM: Sandia National Laboratories.

Hora, S.C., D. von Winterfeldt, and K.M. Trauth. 1991. Expert Judgment on Inadvertent Human Intrusion into the Waste Isolation Pilot Plant. SAND90-3063. Albuquerque, NM: Sandia National Laboratories.

Lappin, A.R. 1988. Summary of Site-Characterization Studies Conducted From 1983 Through 1987 at the Waste Isolation Pilot Plant (WIPP) Site, Southeastern New Mexico. SAND88-0157. Albuquerque, NM: Sandia National Laboratories.

Marietta, M.G., S.G. Bertram-Howery, D.R. Anderson, K.F. Brinster, R.V. Guzowski, H. Iuzzolino, and R.P. Rechard. 1989. Performance Assessment Methodology Demonstration: Methodology Development for Evaluating Compliance with EPA 40 CFR Part 191, Subpart B, for the Waste Isolation Pilot Plant. SAND89-2027. Albuquerque, NM: Sandia National Laboratories.

Public Law'96-164. 1979. Department of Energy National Security and Military Applications of Nuclear Energy Authorization Act of 1980. 
Public Law 102-579. 1992. Waste Isolation Pilot Plant Land Withdrawal Act.

Rechard, R.P. 1989. Review and Discussion of Code Linkage and Data Flow in Nuclear Waste Compliance Assessments. SAND87-2833. Albuquerque, NM: Sandia National Laboratories.

Rechard, R.P., K.M. Trauth, and R.V. Guzowski. 1992. Quality Assurance Procedures for Parameter Selection and Use of Expert Judgment Panels Supporting Performance Assessments of the Waste Isolation Pilot Plant. SAND91-0429. Albuquerque, NM: Sandia National Laboratories.

U.S. DOE (Department of Energy). 1980. Waste Isolation Pilot Plant Safety Analysis Report. Washington, DG: U.S. Department of Energy. Vols. 1-5.

U.S. DOE (Department of Energy) and the State of New Mexico. 1981, as modified. "Agreement for Consultation and Cooperation" on WIPP by the State of New Mexico and.U.S. Department of Energy, modified 11/30/84 and $8 / 4 / 87$.

U.S. EPA (Environmental Protection Agency). 1985. "Environmental Standards for the Management and Disposal of Spent Nuclear Fuel, High-Level and Transuranic Radioactive Wastes: Final Rule, 40 CFR Part 191," Federal Register. Vol. 50, no. 182, 38066-38089.

U.S. NRC (Nuclear Regulatory Commission). 1990. Severe Accident Risks: An Assessment of Five U.S. Nuclear Power Plants. Final Summary Report. NUREG-1150. Washington, DC: U.S. Nuclear Regulatory Commission. Vol. 1.

Waste Management Technology Department. 1987. The Scientific Program at the Waste Isolation Pilot Plant. SAND85-1699. Albuquerque, NM: Sandia National Laboratories.

Weart, W.D., S.G. Bertram-Howery, R.V. Guzowski, R.F. Brinster, P.N. Swift, and S.B. Pasztor. 1991. Background Information Presented to the Expert Panel on Inadvertent Human Intrusion into the Waste Isolation Pilot Plant. Eds. R.V. Guzowski and M.M. Gruebel. SAND91-0928. Albuquerque, NM: Sandia National Laboratories. 
APPENDIX A: ISSUE STATEMENT FOR THE DEVELOPMENT OF MARKERS TO DETER INADVERTENT HUMAN INTRUSION INTO THE WASTE ISOLATION PILOT PLANT 
A-2 


\title{
APPENDIX A: ISSUE STATEMENT FOR THE DEVELOPMENT OF MARKERS TO DETER INADVERTENT HUMAN INTRUSION INTO THE WASTE ISOLATION PILOT PLANT
}

\author{
THE DEVELOPMENT OF MARKERS TO DETER INADVERTENT HUMAN \\ INTRUSION INTO THE WASTE ISOLATION PILOT PIANT
}

\section{ISSUE STATEMENT}

The Waste Isolation Pilot Plant (WIPP) could become an underground disposal system for wastes contaminated with transuranic (heavier than uranium) radionuclides from defense activities. The WIPP is located in southeastern New Mexico, near Carlsbad, in bedded salt $2150 \mathrm{ft}$. beneath the earth's surface. Experts will consider passive markers for deterring inadvertent human intrusion, defining characteristics for selecting and manufacturing markers to be placed at the WIPP, and judging the performance of these markers over a 10,000 year period. A marker is something interpretable by the human mind that bears an explicit or implicit message. After

installation, passive markers should remain operational without further human attention.

The current interpretation of the Standard (40 CFR Part 191) is that the characteristics should be designed so that during the ten thousand year performance period, the markers and their message(s) will have a high probability of warning potential intruders of the dangers associated with the transuranic wastes held within the repository, as well as their location. A system of several types of markers is an acceptable response to this issue statement.

Once the marker characteristics have been defined, the likely future performance of these markers as deterrents to various kinds of intrusions will be judged. Such judgments are dependent upon the possible future states of society and on the physical changes that the region surrounding the WIPP could undergo. The teams of experts who have studied these futures as part of this project have identified various plausible futures including the possible characteristics of future societies, the potential modes of inadvertent intrusion, and the frequencies of these inadvertent intrusions. In order to provide deterrence, the markers must be recognized, their meaning correctly interpreted, and they must elicit the desired action from potential intruders.

The specific questions that the experts are asked to address follow. These questions are related to design considerations, performance of individual markers, and performance of the entire system.

\section{Marker Design Characteristics}

Address each of the following, considering the collection of futures presented by the group identifying future societies and possible modes of . inadvertent human intrusion.

1. What markers should be used to mark the WIPP disposal system? This question asks for a general description of the marker system. The details of the markers are asked for in the ensuing questions. Note that the system may consist of more than one type of marker. 


\section{Marker Characteristics}

For each of the markers described in the answer to question 1 , consider:

2. The physical description of the markers including size, location, shape, and materials.

3. The messages upon or in the markers and the method of conveying the messages.

\section{Performance of the System of Markers}

The impact of markers in deterring inadvertent intrusion may not be independent. Nonetheless, an evaluation of the performance of individual markers could be useful to future decision makers in selecting markers if the entire system cannot be put in place, and in selecting between the markers recommended by the two teams.

For each of the major modes of intrusion:

4. Judge the likelihood (as'a function of time) that each marker has persisted to the extent that it is recognizable as such and its message is apparent.

5. Given that a marker has survived, what is the likelihood that each civilization engaging in each specific potential intrusion will recognize the message and correctly interpret that message.

6. Given that the marker has survived and that the message has been correctly interpreted, what is the likelihood that the civilization engaging in each specific potential intrusion will take appropriate action given the message.

Finally, for the system of markers:

7. For the system of markers described above, judge the likelihood that the system persists (as in question 4), the message is correctly interpreted (as in question 5), and intrusion is deterred (as in question 6).

Questions 5, 6, and, in part, 7 require assessments of how future societies will comprehend the markers and their messages. Because the characteristics of these future societies are very uncertain, you are asked to respond to these questions taking into account a wide range of future societies. If this task is too difficult, assessments may be made for several representative societies. For example, societies that are more advanced and less advanced than our society and societies that are similar to present day society may be considered. If assessments conditional on various societies are made, it will be necessary to provide the likelihoods of the various societies. Guidance in assigning the probabilities of the various societies can be found in the report from the Future Intrusion Panel in the form of the societies and probabilities they developed and their rationale. The probabilities cannot be obtained directly from this study, however, because each team provided alternative interpretations of the various future societies.

The work of the Futures Intrusion Panel highlighted a number of modes of inadvertent human intrusion for which markers at the WIPP may provide deterrence. They fall into the two general categories of boreholes and 
excavations. In the first category, the boreholes may be drilled for hydrocarbon exploration and extraction, water wells, or waste injection. In the second category, there may be archaeological excavations or other scientific excavations. Within an excavation project, it is conceivable that there may also be drilling activities. Depending on the particular use of the borehole or excavation, there may be different motivations for intruding and perhaps different numbers of intrusions. The Panel is free to address deterrence for each type of intrusion separately or for a category.

As the above questions are asked as a function of time, it is convenient to divide the entire 10,000 year time period into the near, medium, and far futures. Specifically, you are asked to consider 0 to 500 years, 500 to 2,000 years, and 2,000 to 10,000 years. The near future represents the general time period during which society might still be based on hydrocarbon usage (as discussed in some of the Future Intrusion Panel team reports). The medium future represents a period during which markers might be more likely to survive and be interpretable. The far future represents a period when there may be a lower probability that markers will survive and be interpretable.

\section{Framework for the Expert Judgments}

The work of the Marker Development Panel is part of a staged process to develop markers for the WIPP. It is therefore necessary for the Panel to work within the confines of the work done previously and the performance assessment requirements.

\section{Marker Design Characteristics}

The Panel is free to recommend a "no marker" strategy or any other marking strategy.

If a "no marker" strategy is recommended, the Panel must still recommend the best system of markers as the current Standard (40 CFR Part 191) states that markers will be used.

\section{Performance of the System of Markers}

The results must be applicable to the modes and probabilities of intrusion developed by the Future Intrusion Panel.

Additional future societies, modes of intrusion, and probabilities of intrusion that a team wishes to develop for consideration in the design criteria and effectiveness judgments should be contained in the team report. These three items, as well as the effectiveness of the markers in deterring these intrusions can be elicited, if necessary, at the second meeting of the Marker Development Panel.

\section{Communication of Findings}

We ask that each team provide responses to the above questions and the rationales supporting these responses. The responses should be in the form of a draft report that includes a description of the recommended marker system, and factors that would impact the effectiveness of various markers in deterring various types of intrusions, as well as the assumptions, methods, rationales, and other information used to reach these conclusions. The draft report should be finalized after the second meeting, after the 
judgments have been elicited, and there has been interaction between the two teams.

The assessment of the probabilities of: 1) existence, 2) interpretation, and 3 ) deterrence, as well as possible modification of the design criteria will take place during the second meeting of the teams. Each team of experts is expected to make a presentation of their findings to the other team and the project staff. Similarly, while the teams are asked to develop/identify factors influencing marker effectiveness, the assessment of probabilities will be accomplished during the second meeting. This is not to say that the expert participants should not give deep and careful consideration to the assignment of these probabilities, however. The intention here is to preclude the fixing of positions until after an exchange of ideas takes place between the two teams. Further, it is desired that the actual assessment of probabilities be done in conjunction with the decision analysts participating in this project.

The probability assessments of the experts will be documented and processed, and returned to the experts for comment and review. Following concurrence by the experts, the results will be summarized and conveyed to the DOE and the WIPP performance assessment team for inclusion in the performance calculations of the WIPP system. 


\section{APPENDIX B: LETTER REQUESTING NOMINATIONS}

B-1 
B - 2 


\section{APPENDIX B: LETTER REQUESTING NOMINATIONS}

\section{Sandia National Laboratories}

Albuquerque. New Mexico 87185

July 24,1990

$$
\begin{aligned}
& <\mathrm{fn}><\ln > \\
& <\mathrm{co}> \\
& <\mathrm{jt}> \\
& <\text { add1 }> \\
& <\text { add2 }> \\
& <\text { add3> } \\
& <\mathrm{ct}>,<\mathrm{st}><\text { zip }> \\
& \text { Dear }<\mathrm{ti}><\ln >\text { : }
\end{aligned}
$$

The safe disposal of nuclear waste is one of the most pressing issues facing the United States today. The Waste Isolation Pilot Plant (WIPP), located in New Mexico, is to be the first of this nation's nuclear waste repositories. The geologic and hydrologic properties of the site indicate that the WIPP system will serve as an effective repository, if left undisturbed. Inadvertent human intrusion, however, might result in radioactive releases to the biosphere. Preventing such intrusion through the development and implementation of a passive marker system that will deter inadvertent human intrusion into the repository is essential for assessing the performance of the site. We seek your assistance in nominating persons to participate in the identification of characteristics for selecting and manufacturing markers to be placed at the WIPP site. Participants in this study will identify the possible physical properties of markers (both composition and placement), as well as the form and content of the messages such markers should carry. If your qualifications are appropriate for this study, we encourage you to place your own name in nomination.

Because the knowledge necessary to develop a marker system that will remain operational during the performance period of the site $(10,000$ years) can be found across many of our traditional disciplines of study, we are attempting to construct panels that are multidisciplinary in nature--spanning the fields of materials science, climatology, communications, and the social sciences including archaeology, anthropology, and psychology.

Attached is a more detailed description of the tasks to be accomplished. While the total effort required from the various panel members may vary because of their backgrounds and areas of responsibility, we envision a commitment of about three weeks effort including two meetings to be held in New Mexico on October 10-12, and December 13-14, 1990. Expenses and an honorarium in lieu of professional fees will be provided by Sandia National Laboratories. 
Please send your nominations to me by August 3, 1990. We request that, if possible, you contact suggested nominees to inform them of your nomination and verify their interest in participating. Your inclusion of complete addresses and telephone numbers (both voice and FAX if available) will be greatly appreciated. We will contact the nominees shortly thereafter and request credentials. The selection of participants will be based on tangible evidence of expertise, previous work in related areas, availability, and freedom from conflicts of interest.

If you need additional information, please contact Mr. Dan Scott or Ms. Suzanne Pasztor at (505) 844-1917. If you wish, you may send your nominations by FAX to Mr. Scott or Ms. Pasztor at (505) 844-1723 or you may mail them directly to me.

Thank you for your assistance with this important issue.

Sincerely,

D. R, hindingom

D. Richard Anderson

Performance Assessment

Division 6342

Sandia National Laboratories

Albuquerque, NM 87185

Enclosure 


\section{ACQUISITION OF EXPERT JUDGMENTS FOR PASSIVE MARKERS TO DETER HUMAN INTRUSION INTO NUCLEAR WASTE REPOSITORIES}

The Waste Isolation Pilot Plant (WIPP) is an underground storage facility for transuranic (heavier than uranium) radioactive wastes from military weapons production. The repository is located in southeastern New Mexico, near Carlsbad, in bedded salt approximately $2500 \mathrm{ft}$. beneath the earth's surface. The goal of the expert judgment effort for passive markers to deter human intrusion is the creation of characteristics for selecting and manufacturing markers to be placed at the WIPP site. A marker is something detectable by the mind that bears an explicit or implicit message. Passive markers remain operational without further human attention.

Design characteristics can be implemented in various ways. For example, the design characteristics may specify that the markers shall be placed so that they delineate the surface area above the repository. Those markers located to each side of any one marker can be seen with the unaided eye from that marker. Further, the weight of the marker, or each piece of the marker, shall be great enough to discourage removal and the marker's overall height (above and below the ground) shall not only enhance ground and aerial visibility, but ensure stability with respect to anticipated erosion and prevent burial due to shifting sands and soil. The monuments shall be made of a durable material known to withstand the weathering under current conditions at the WIPP site for ten thousand years. The material shall not have value as a resource.

The messages on the monuments would be further described in the characteristics. For example, the characteristics may state that the message must be provided in the form of a pictograph, and in English, Chinese, and Russian. The contents of the message would clearly signal the presence of biohazardous waste in the repository. Further, the message will be inscribed to a depth sufficient to prevent obliteration by erosion or corrosion.

The characteristics should be designed so that during the ten thousand year performance period, the markers will have a high probability of warning inadvertent intruders of the dangers associated with the transuranic wastes held within the repository. A system of several types of markers may perform better than a single type of marker for several reasons. First, there are events that may yield certain types of markers ineffective while not impairing other types of markers. For instance, monuments may be covered by sand dunes while buried magnetic markers may remain detectable. Second, some types of markers may be more effective for specific types of intrusions. Brilliantly colored layers of materials laid beneath the surface may provide a warning when brought to the surface by conventional drilling, but might go unnoticed when drilling is performed by lasers.

The creation of characteristics for markers, then, requires the assessment of specific marker performance for various modes of intrusion under various scenarios of natural and manmade processes that may destroy or neutralize the 
markers. The study of intrusion modes will be completed at an earlier stage in the overall project and will be provided as an input to the group working on characteristics for markers. The expert group may, however, develop additional intrusion modes.

The expert group will formulate characteristics for passive markers to provide a high probability of effective warning at various times in the future. A marker can provide an effective warning only if:

1. It survives. 2. It can be detected. 3. The message is perceived as a warning.

4. The warning initiates appropriate action.

The expert group must consider each of these aspects in developing characteristics.

A presentation will be made to the group of experts on the background research on markers performed in predecessor studies of radioactive waste disposal. This review will take place in New Mexico October 10-12 of this year at a location to be determined. The group will be introduced to the techniques and procedures of probability elicitation and will undergo training in probability assessment. Finally, the group will be taken on a familiarization tour of the WIPP site which is located in Carlsbad, New Mexico.

The experts will then be asked to spend several weeks over the following two month period preparing analyses using the tools and knowledge of their own disciplines. The group will reassemble December 13-14, 1990 to make presentations of their findings. Written documentation in the form of a paper or report will be furnished as part of the presentation. Next, the experts will be asked to provide probabilistic assessments of the performance of various types of markers. These assessments will be collected using the methods of formal probability elicitation.

The probability assessments of the experts will be documented and processed and returned to the experts for comment and review. Following concurrence by the experts, the results will be summarized and conveyed to the DOE and the WIPP performance assessment team for inclusion in the performance calculations of the WIPP system.

Expenses and an honorarium in lieu of professional fees will be paid by the project. 
APPENDIX C: LIST OF THOSE TO WHOM REQUESTS FOR NOMINATIONS WERE SENT 
C-2 


\section{APPENDIX C: LIST OF THOSE TO WHOM REQUESTS FOR NOMINATIONS WERE SENT}

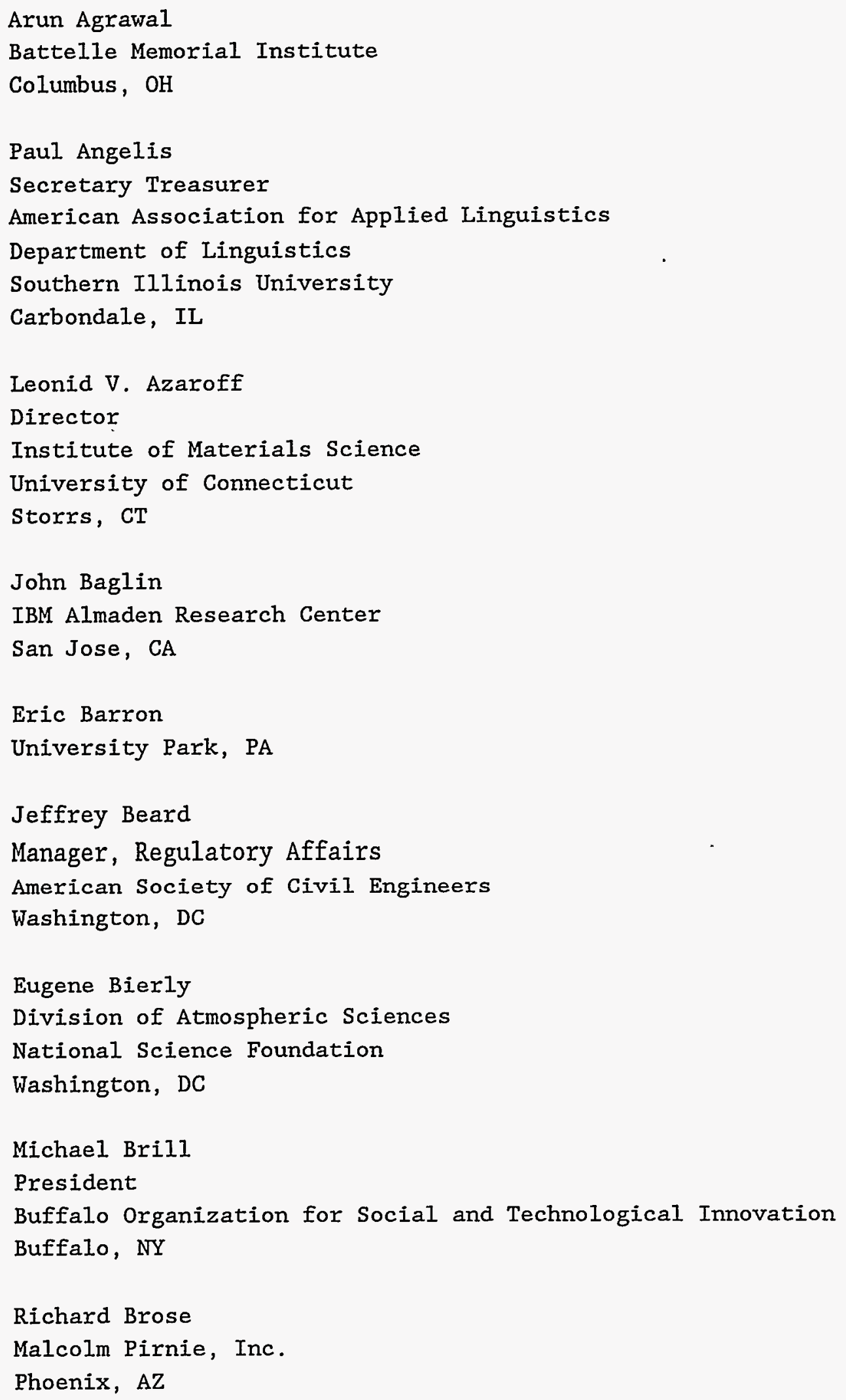


Austin Chang

Department of Materials Science and Engineering

University of Wisconsin

Madison, WI

David E. Glement

Columbia, SC

John E. Costa

United States Geological Survey

Vancouver, WA 98661

Bob Costello

Hudson Institute

Indianapolis, IN

Donna Kelleher Darden

Department of Sociology

Eckerd College

St. Petersburg, FL

Leonard David

Space Data Resources

Washington, DC

Frank Drake

Lick Observatory

University of California

Santa Cruz, CA

Charles Fairhurst

Department of Civil and Mineral Engineering

University of Minnesota

Minneapolis, $\mathrm{MN}$

Virginia Fairweather

Editor, CiviI Engineering

New York, NY

Harry Farrar, IV

Rockwell International

Canoga Park, CA 


\author{
Barry Fell \\ President \\ Epigraphic Society \\ San Diego, CA \\ Ben Finney \\ Department of Anthropology \\ University of Hawaii \\ Honolulu, HI \\ Susan Gass \\ English Language Center \\ Michigan State University \\ East Lansing, MI \\ Richard L. Gay \\ Rockwell International \\ Canoga Park, CA \\ Carol Gipp \\ Project Coordinator \\ National Congress of American Indians \\ Washington, DC \\ David Givens \\ American Anthropological Association \\ Washington, DC \\ Thomas Greaves \\ Executive Director \\ Bucknell University \\ Lewisburg, PA \\ Ken Hadeen \\ National Climatic Data Center \\ Ashville, NC \\ Don Hancock \\ Southwest Research and Information Center \\ Albuquerque, NM
}


J.N. Hartley

Battelle Pacific Northwest Laboratory

Environmental Management Operations

Richland, WA

Richard Henshel

Department of Sociology

University of Western Ontario

London, Canada

Jack Howel1

Associate Executive Director of Programs

American Society of Mechanical Engineers

New York, NY

Paul Kay

Secretary-Treasurer

Society for Linguistic Anthropology

Department of Linguistics

University of California, Berkeley

Berkeley, CA

Maureen F. Kaplan

Eastern Research Group, Inc.

Lexington, MA

Max Lagally

Department of Material Science and Engineering

University of Wisconsin

Madison, WI

T.G. Langdon

Department of Materials Science

University of Southern California

Los Angeles, CA

Richard Lanigan

Speech Communications Department

Southern Illinois University

Carbondale, IL

Clifford F. Lewis

Editor, Materials Engineering

Cleveland, $\mathrm{OH}$ 
Christopher D. Lidstone

Lidstone and Anderson, Inc.

Fort Collins, $\mathrm{CO}$

Jon Lomberg

Honaunau, HI

Dean MacConne11

Acting Executive Director

Semiotic Society of America

Applied Behavioral Science

University of California, Davis

Davis, CA

Harris Marcus

Director, Center for Research in Materials Science and Engineering

University of Texas at Austin

Austin, $\mathrm{TX}$

Tom May

Director

Environmental Design Research Associates

Oklahoma City, OK

Tom McCulloch

Advisory Council on Historic Preservation

Washington, DC

William S. Neubeck

Feura Bush, NY

Dennis N. Nielsen

Dean

College of Science and Engineering

Winona State University

Winona, MN

S.J. Phillips

Westinghouse

Richland, WA

Ken Pierce

United States Geological Survey-Denver

Denver, CO 
Russell Pinizzotti

University of North Texas

Denton, TX

Jeffrey K. Polum

Dean of the Graduate School

University of Santa Cruz

Santa Cruz, CA

Robert Quayle

National Climatic Data Center

Ashville, NC

C.C. Reeves

Department of Geosciences

Texas Tech University

Lubbock, TX

Marilyn Reeves

League of Women Voters

Amity, Oregon

Richard Reisenweber

Vice President, Environmental Control

Rockwell International

E1 Segundo, CA

Margaret Reynolds

Executive Director

Linguistic Society of America

Washington, DC

Thomas E. Rice

Amherst, MA

Richard Ricker

Corrosion Group Leader

National Institute of Standards and Technology

Gaithersburg, MD

Reed D. Riner

Flagstaff, AZ 


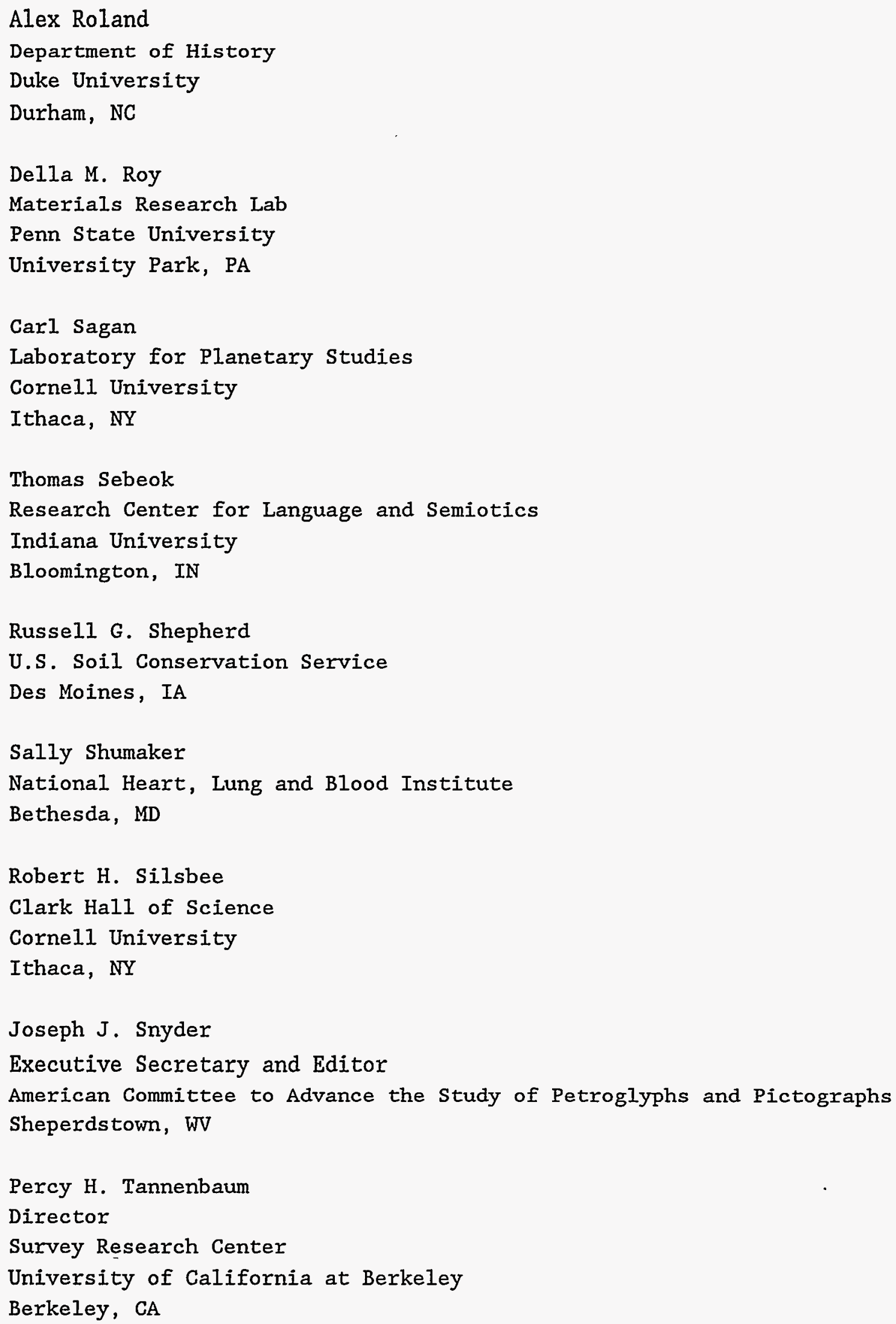


G. Richard Tucker

President

Center for Applied Linguistics

Washington, DC

William J. Wayne

Department of Geology

University of Nebraska

Lincoln, NE

Julie $P$. Weatherington-Rice

Bennett and Williams, Inc.

Worthington, $\mathrm{OH}$

Abraham Weitzberg

NUS Corporation

Rockville, MD

Gerry Wendorf

Department of Anthropology

Southern Methodist University

Dallas, TX

Larry Whipple

Managing Director for Professional Affairs

American Society of Civil Engineers

New York, NY

Ray A. Williamson

Space Policy Institute

George Washington University

Washington, DC

Thomas G. Winner

Barnet, VT 
APPENDIX D: LETTER TO NOMINEES

D-1 


$$
\text { D-2 }
$$




\section{APPENDIX D: LETTER TO NOMINEES}

\section{Sandia National Laboratories}

Albuquerque. New Mexico 87185

July 23, 1990

\section{Dear}

The safe disposal of nuclear waste is one of the most pressing issues facing the United States today. The Waste Isolation Pilot Plant (WIPP), located in New Mexico, is to be the first of this nation's nuclear waste repositories. The geologic and hydrologic properties of the site indicate that the WIPP system will serve as an effective repository, if left undisturbed. Inadvertent human intrusion, however, might result in radioactive releases to the biosphere. Sandia National Laboratories is working on the development and implementation of a passive marker system to deter inadvertent human intrusion into the repository.

You have been nominated to participate in a study sponsored by Sandia National Laboratories that will identify what kinds of markers should be placed at the WIPP site and will attempt to assess the effectiveness of such a marking system. A brief description of the problem, the criteria for selecting participants from the nominees, and scheduling information follow.

The knowledge necessary to develop a marker system that will remain operational during the performance period of the site-10,000 years-can be found across many of our traditional disciplines of study. For this reason, we are constructing a panel of eight to ten experts that is multidisciplinary in nature-spanning the fields of materials science, climatology, communications, and the social sciences including archaeology, anthropology, and psychology. Each panel member will answer questions regarding the marker system that directly concern his or her expertise. For example, a materials scientist will help identify what the markers should be made of, while a linguist will be concerned with what kind of inscription should go on the markers.

Attached is a more detailed description of the tasks to be accomplished. While the total effort required from the various team members may vary because of their backgrounds and areas of responsibility, we envision a commitment of about three weeks effort including two meetings to be held in New Mexico: one during early fall (October 10 through 12) and another two months following the first meeting (December 13 and 14). Expenses and an honorarium in lieu of professional fees will be provided by Sandia National Laboratories. 
If you are interested in serving on this project, please send me your resume and a letter stating your interest by August 15, 1990. This letter should include a brief description of why you feel you are qualified to serve. Citing work you have accomplished that is germane to this study would be helpful to our selection committee. You should also show that you will be able to attend the required meetings and perform the assigned work between the two meetings. The selection of participants will be based on tangible evidence of expertise, curriculum vitae, previous work in related areas, availability, and freedom from conflicts of interest.

If you need additional information, please contact Mr. Dan Scott or Ms. Suzanne Pasztor at (505) 844-1917. If you wish, you may send your letter requesting to serve on the study by fax to Mr. Scott or Ms. Pasztor at (505) 844-1723, or you may mail them directly to me.

Thank you for your assistance with this important issue.

\author{
Sincerely, \\ D. R. Anderoor \\ D. Richard Anderson \\ Performance Assessment \\ Division 6342 \\ Sandia National Laboratories \\ Albuquerque, NM 87185
}

Enclosure 


\section{ACQUISITION OF EXPERT JUDGMENTS FOR PASSIVE MARKERS TO DETER HUMAN INTRUSION INTO NUCLEAR WASTE REPOSITORIES}

The Waste Isolation Pilot Plant (WIPP) is an underground storage facility for transuranic (heavier than uranium) radioactive wastes from military weapons production. The repository is located in southeastern New Mexico, near Carlsbad, in bedded salt approximately $2500 \mathrm{ft}$. beneath the earth's surface. The goal of the expert judgment effort for passive markers to deter human intrusion is the creation of characteristics for selecting and manufacturing markers to be placed at the WIPP site. A marker is something detectable by the mind that bears an explicit or implicit message. Passive markers remain operational without further human attention.

Design characteristics can be implemented in various ways. For example, the design characteristics may specify that the markers shall be placed so that they delineate the surface area above the repository. Those markers located to each side of any one marker can be seen with the unaided eye from that marker. Further, the weight of the marker, or each piece of the marker, shall be great enough to discourage removal and the marker's overall height (ábove and below the ground) shall not only enhance ground and aerial visibility, but ensure stability with respect to anticipated erosion and prevent burial due to shifting sands and soil. The monuments shall be made of a durable material known to withstand the weathering under current conditions at the WIPP site for ten thousand years. The material shall not have value as a resource.

The messages on the monuments would be further described in the characteristics. For example, the characteristics may state that the message must be provided in the form of a pictograph, and in English, Chinese, and Russian. The contents of the message would clearly signal the presence of biohazardous waste in the repository. Further, the message will be inscribed to a depth sufficient to prevent obliteration by erosion or corrosion.

The characteristics should be designed so that during the ten thousand year performance period, the markers will have a high probability of warning inadvertent intruders of the dangers associated with the transuranic wastes held within the repository. A system of several types of markers may perform better than a single type of marker for several reasons. First, there are events that may yield certain types of markers ineffective while not impairing other types of markers. For instance, monuments may be covered by sand dunes while buried magnetic markers may remain detectable. Second, some types of markers may be more effective for specific types of intrusions. Brilliantly colored layers of materials laid beneath the surface may provide a warning when brought to the surface by conventional drilling, but might go unnoticed when drilling is performed by lasers.

The creation of characteristics for markers, then, requires the assessment of specific marker performance for various modes of intrusion under various scenarios of natural and manmade processes that may destroy or neutralize the markers. The study of intrusion modes will be completed at an earlier stage in the overall project and will be provided as an input to the group working on characteristics for markers. The expert group may, however; consider additional intrusion modes.

The expert group will formulate characteristics for passive markers to provide a high probability of effective warning at various times in the future. A marker can provide an effective warning only if: 
1. It survives.

2. It can be detected.

3. The message is perceived as a warning.

4. The warning initiates appropriate action.

The expert group must consider each of these aspects in developing characteristics.

A presentation will be made to the group of experts on the background research on markers performed in predecessor studies of radioactive waste disposal. This review will take place in New Mexico October 10-12 of this year in Albuquerque, New Mexico. The group will be introduced to the techniques and procedures of probability elicitation and will undergo training in probability assessment. Finally, the group will be taken on a familiarization tour of the WIPP site which is located in Carlsbad, New Mexico.

The experts will then be asked to spend several weeks over the following two month period preparing analyses using the tools and knowledge of their own disciplines. The group will reassemble December 13-14, 1990 to make presentations of their findings. Written documentation in the form of a paper or report will be furnished as part of the presentation. Next, the experts will be asked to provide probabilistic assessments of the performance of various types of markers. These assessments will be collected using the methods of formal probability elicitation.

The probability assessments of the experts will be documented and processed and returned to the experts for comment and review. Following concurrence by the experts, the results will be summarized and conveyed to the DOE and the WIPP performance assessment team for inclusion in the performance calculations of the WIPP system.

Expenses and an honorarium in lieu of professional fees will be paid by the project. 
APPENDIX E: EXPERT PANEL SELECTION CRITERIA

E-1 


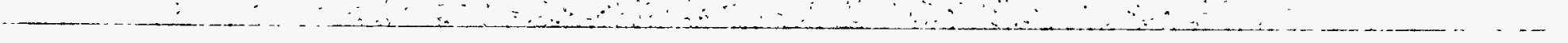




\section{APPENDIX E: EXPERT PANEL SELECTION CRITERIA}

Each member of the selection committee evaluated the nominees based on the following criteria:

tangible evidence of expertise,

professional reputation,

availability and willingness to participate,

understanding of the general problem area,

impartiality,

lack of economic or personal stake in the potential findings,

balance among team members so that each team has the needed breadth of expertise,

physical proximity to other participants so that teams can work effectively,

balance among all participants so that various constituent groups are represented. 


\section{APPENDIX F: TEAM A REPORT: MARKING THE WASTE ISOLATION PILOT PLANT FOR 10,000 YEARS}

Prepared by Team A:

Dieter G. Ast

Michael Brill

Ward Goodenough

Maureen Kaplan

Frederick Newmeyer

Woodruff Sullivan

April 1992 
F-2 
APPENDIX F: TEAM A REPORT: MARKING THE WASTE ISOLATION PILOT PLANT FOR 10,000 YEARS

\title{
MARKING THE WASTE \\ ISOLATION PILOT PLANT \\ FOR 10,000 YEARS
}

\author{
Prepared by: \\ Dieter G. Ast \\ Michael Brill \\ Ward Goodenough \\ Maureen Kaplan \\ Frederick Newmeyer \\ Woodruff Sullivan \\ ['TEAM A']
}

April 1992

A Report prepared for the US Department of Energy 


\section{Table of Contents}

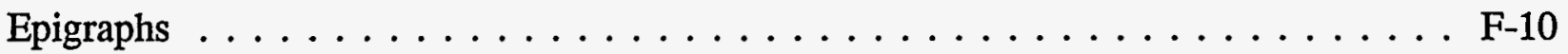

EXECUTIVE SUMMARY $\ldots \ldots \ldots \ldots \ldots \ldots \ldots \ldots \ldots$ F-11

1. General Considerations

1.1 Background information on marking the WIPP site $\ldots \ldots \ldots \ldots \ldots$ F-19

1.2 Charge to the expert panel $\ldots \ldots \ldots \ldots \ldots \ldots \ldots \ldots \ldots \ldots$ F-20

1.3 Should the site be marked? ................... F-23

1.3.1 Motivations for Marking . . . . . . . . . . . . . F-23

1.3.2 General Criteria for any Marking System . . . . . . . . . . . F-24

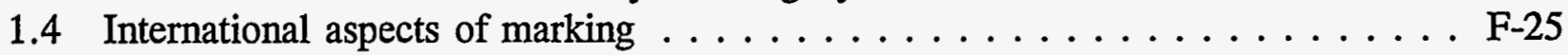

1.5 A system approach...two major themes $\ldots \ldots \ldots \ldots \ldots \ldots \ldots \ldots$ F-28

1.6 On-site testing of markers $\ldots \ldots \ldots \ldots \ldots \ldots \ldots \ldots \ldots \ldots$ F-30

1.7 References ......................... F-31

2. The Problem of Message

2.1 Message definition $\ldots \ldots \ldots \ldots \ldots \ldots \ldots \ldots \ldots \ldots \ldots$ F-33

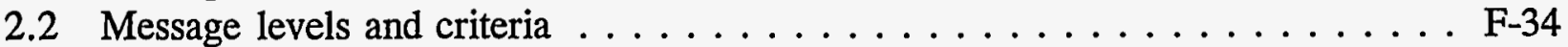

2.2.1 Message Levels . . . . . . . . . . . . . . . . . F-34

2.2 .2 Criteria . . . . . . . . . . . . . . . . F-35

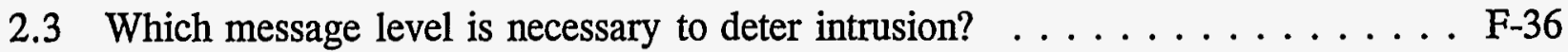

2.3.1 Activities Near the Site $\ldots \ldots \ldots \ldots \ldots \ldots \ldots \ldots \ldots \ldots$ F-36

2.3.2 Activities at the Site $\ldots \ldots \ldots \ldots \ldots \ldots \ldots \ldots \ldots \ldots$ F-37

2.4 References . . . . . . . . . . . . . . . . . . F-37

3. Components of a Marking System

3.1 Communications: site and structures $\ldots \ldots \ldots \ldots \ldots \ldots$. . . . . . F-39

3.2 General discussion: for the site as a whole and individual

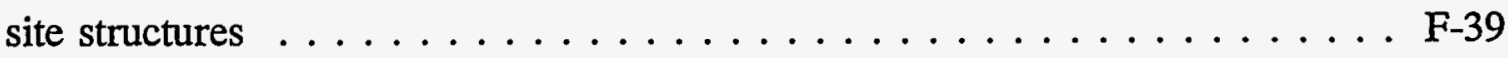


3.2.1 The Concept of Archetypes . . . . . . . . . . . . . . . F-40

3.2.2 Archetypes Operating as a Natural Language . . . . . . . . . . F F-40

3.2.3 The Medium for Expression of Place-Archetypes . . . . . . . . . F-41

3.3 The appropriate use of graphics in marking $\ldots \ldots \ldots \ldots \ldots \ldots \ldots \ldots$ F-42

3.3 .1 Ambiguity . . . . . . . . . . . . . . . . F F-42

3.3 .2 Removal ........................F-43

3.3.3 Cultural Restrictions $\ldots \ldots \ldots \ldots \ldots \ldots \ldots \ldots \ldots \ldots$. . . . . . . .

3.3.4 Universal Expressions . . . . . . . . . . . . . . . . . F-44

3.4 The languages to be inscribed on markers $\ldots \ldots \ldots \ldots \ldots \ldots \ldots$ F-44

3.4.1 The Importance of Linguistic Markings . . . . . . . . . . . . . F-44

3.4.2 Which Languages Should be Chosen? . . . . . . . . . . . . . . . F-44

3.4.2.1 Linguistic Demography of the WIPP Site . . . . . . . . F F-44

3.4.2.2 The Choice of Languages . . . . . . . . . F-45

3.5 Public information at the WIPP site . . . . . . . . . . . . F F-46

3.5.1 Public Involvement in Marking and Publicizing

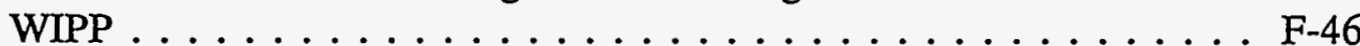

3.5 .2 Off-site Archiving $\ldots \ldots \ldots \ldots \ldots \ldots \ldots \ldots \ldots$ F-46

3.5.3 Empty Space for Reinscription $\ldots \ldots \ldots \ldots \ldots \ldots \ldots \ldots \ldots$ F-47

3.6 References . . . . . . . . . . . . . . . . . . F

4. Criteria for a Marking System with Examples

4.1 Site design guidelines for a design of the entire site, so it is

a major component of a system of messages . . . . . . . . . . F-49

4.2 Design options $\ldots \ldots \ldots \ldots \ldots \ldots \ldots \ldots \ldots \ldots \ldots \ldots$ F-57

4.3 A visual depiction of various design options . . . . . . . . . . F F-60

4.4 Durability of common marker structures $\ldots \ldots \ldots \ldots \ldots \ldots \ldots \ldots \ldots$ F-79

4.4 .1 Introduction $\ldots \ldots \ldots \ldots \ldots \ldots \ldots \ldots \ldots \ldots \ldots \ldots \ldots \ldots$

4.4.2 Should all Markers be Durable? . . . . . . . . . . . . . . F-79

4.4.3 Categories of Markers . . . . . . . . . . . . . . F-80

4.4.4 Distinction Between Markers and Barriers . . . . . . . . . . F-80

4.4.5 Five Principles to Maximize Durability $\ldots \ldots \ldots \ldots \ldots \ldots \ldots$ F-80

4.4 .6 Environmental Factors . . . . . . . . . . . . . . . . F-89

4.4.7 Feasibility Demonstration that Durable Markers can
be Constructed...if Cost is No Object . . . . . . . . . . . . F-90

4.4.7.1 Longevity Principles in the Classical

Pyramid Design . . . . . . . . . . . . . . F-90

4.4.7.2 Shortcomings of a Pyramid Design . . . . . . . . . F-92

4.4.8 Minimizing Marker Removal by Humans . . . . . . . . . . . . . F-92

4.4.9 Durability of Some Common Elements of a Marker

System . . . . . . . . . . . . . . . . . . . . F F-97

4.4.9.1 Introduction . . . . . . . . . . . . . F-97

4.4.9.2 Large Earth Berms . . . . . . . . . . . . . . . F-98

4.4.9.3 Monoliths Made of Stone . . . . . . . . . . . . . . . F-98

4.4.9.4 Concrete Monoliths . . . . . . . . . . . . . F-100

4.4.9.5 Composite "Monoliths" . . . . . . . . . . . . . . . F-101 
4.4.9.6 Markers Generating Noise or Tones . . . . . . . . . . F F-101

4.4.9.7 Other Self-Energized Marker Systems . . . . . . . . . . F F-101

4.4.9.8 Above-Ground, Closed Rooms . . . . . . . . . . . F-102

4.4.9.9 Partially Buried, Closed Rooms . . . . . . . . . . F-102

4.4.9.10 Below-Ground, Closed Rooms . . . . . . . . . . . F-105

4.4.9.11 Small-Scale, Near-Surface Markers . . . . . . . . . . F-105

4.4.9.12 Deeply Buried Markers . . . . . . . . . . . . . F-107

4.5 Graphic designs for markers . . . . . . . . . . . . . . F-110

4.5.1 Design Criteria . . . . . . . . . . . . . . . . F F-110

4.5.2 International Symbol for "Buried Radioactive Waste" . . . . . . . . F-112

4.5 .3 Faces . . . . . . . . . . . . . . . . . . F-114

4.5 .4 Maps . . . . . . . . . . . . . . . . . F F-114

4.5.5 Star Map Showing Precession $\ldots \ldots \ldots \ldots \ldots \ldots \ldots \ldots$ F-118

4.5.6 Periodic Table of Elements . . . . . . . . . . . . . F F-120

4.6 Marker messages, Levels II, III, and IV . . . . . . . . . . . . . . . . . F-120

4.6.1 Message Levels, Languages, and Markers . . . . . . . . . . F F-120

4.6.2 The Messages Themselves ................... F-123

4.6.2.1 Marker Message, Level II . . . . . . . . . . . . . . F-123

4.6.2.2 Marker Message, Level III . . . . . . . . . . . . . F F-123

4.6.2.3 Marker Message, Level IV (first alternative) . . . . . . . F-123

4.6.2.4 Marker Message, Level IV (second alternative) . . . . . . F-126

4.7 Additional possible components . . . . . . . . . . . . . F-135

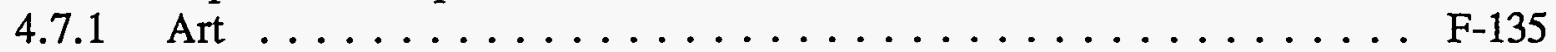

4.7 .2 Aeolian Structures $\ldots \ldots \ldots \ldots \ldots \ldots \ldots \ldots \ldots$ F-136

4.8 References .......................... F-136

5. Appendices

5.1 Scenario for the marking system (MFK) $\ldots \ldots \ldots \ldots \ldots \ldots \ldots \ldots$ F-139

5.2 The enormity of marking the WIPP site (FN) $\ldots \ldots \ldots \ldots \ldots \ldots \ldots$ F-142

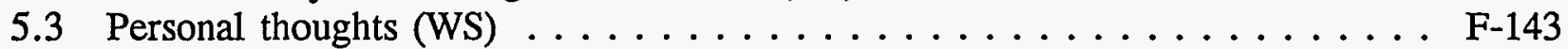

5.4 Possible origins of archetypes of place (MB) $\ldots \ldots \ldots \ldots \ldots \ldots \ldots$. . . . . . . . . F

5.4.1 Landscapes Seen as Having Adaptive Value in Evolution . . . . . . . F-144

5.4.2 Landscapes as Primordial Factor in Development of Mythic

Consciousness ...................... F-145

5.4.3 Archetypes of Built-Form Seen as Originating in Body-

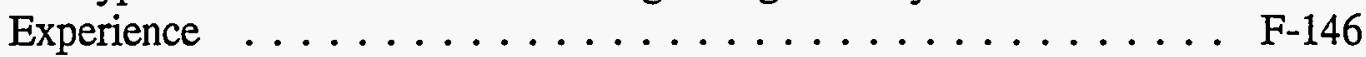

$5.4 .4 \quad$ References . . . . . . . . . . . . . . . . . . . . . F-147

5.5 A Proposal for a Visitors' Center and Memorial at the WIPP

site $(\mathrm{FN}) \ldots \ldots \ldots \ldots$. . . . . . . . . . . . . . . . . F-149

5.6 "Beauty is conserved, ugliness discarded" (DGA) . . . . . . . . . . . F-152

Additional Information from SAND92-1382 Authors . . . . . . . . . . . F-153 


\section{Figures}

1.4-1 World wide distribution of nuclear reactors $\ldots \ldots \ldots \ldots \ldots$. . . . . . F-27

4.3-1 Landscape of Thorns, view $1 \ldots \ldots \ldots \ldots \ldots$. . . . . . . . F-61

4.3-2 Landscape of Thorns, view $2 \ldots \ldots \ldots \ldots \ldots \ldots$. . . . . . . . . F-62

4.3-3 Spike Field, view $1 \ldots \ldots \ldots \ldots \ldots \ldots \ldots \ldots$

4.3-4 Spike Field, view $2 \ldots \ldots \ldots \ldots \ldots \ldots \ldots \ldots \ldots \ldots$

4.3-5 Spikes Bursting Through Grid, view $1 \ldots \ldots \ldots \ldots \ldots$. . . . . . F-65

4.3-6 Spikes Bursting Through Grid, view $2 \ldots \ldots \ldots \ldots \ldots$

4.3-7 Leaning Stone Spikes . . . . . . . . . . . . . . . . . . F-67

4.3-8 Menacing Earthworks, view $1 \ldots \ldots \ldots \ldots \ldots \ldots$. . . . . . . F-68

4.3-9 Menacing Earthworks, view $2 \ldots \ldots \ldots \ldots \ldots \ldots$. . . . . . . . F-69

4.3-10 Black Hole, view $1 \ldots \ldots \ldots \ldots \ldots \ldots \ldots \ldots$. . . . . . . . . . . . . . . . .

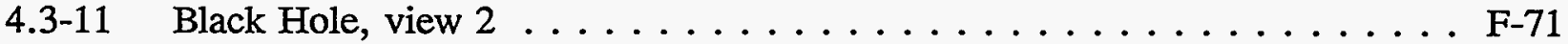

4.3-12 Rubble Landscape, view $1 \ldots \ldots \ldots \ldots \ldots \ldots \ldots \ldots$. . . . . . . . . F-72

4.3-13 Rubble Landscape, view $2 \ldots \ldots \ldots \ldots \ldots \ldots \ldots \ldots$. . . . . . . . . F-73

4.3-14 Forbidding Blocks, view $1 \ldots \ldots \ldots \ldots \ldots \ldots \ldots \ldots \ldots$. . . . . . . . . . . . . . . . . . . . . . .

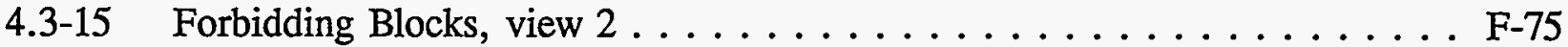

4.3-16 Walk-on Map of World's Radioactive Burial Sties . . . . . . . . . . . F-76

4.3-17 Buried Room with Level IV Messages . . . . . . . . . . . . . . . . F-77

4.3-18 Reading Walls/Message Kiosk . . . . . . . . . . . . . . . . F F-78

4.4-1 Camel Skeleton: Initial discovery, cervical vertebra . . . . . . . . . . F-82

4.4-2 Camel Skeleton: Pre-excavation . . . . . . . . . . . . . . . . . F-82

4.4-3 Camel Skeleton: Overburden removed-excavation begins . . . . . . . . . F-83

4.4-4 Camel Skeleton: Camel being exposed-soft sand . . . . . . . . . . . F F-83

4.4-5 Great Pyramids at Giza: Mycerinus, Chephren, and Cheops . . . . . . . . F-85

4.4-6 The Great Sphinx at Giza . . . . . . . . . . . . . . . . . . F F-86

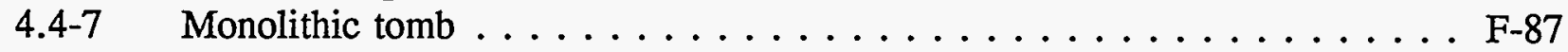

4.4-8 Passage grave ... . . . . . . . . . . . . . . . . . . . F-88

4.4-9 Pyramid of engraved blocks: An example of durable message transmittal . . F-91

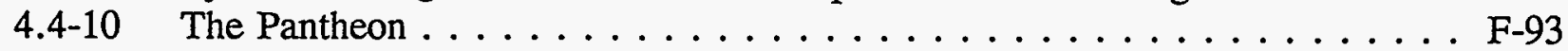

4.4-11 Stonehenge . . . . . . . . . . . . . . . . . . . F-96

4.5-1 Possible prototypes for facial icons, example $1 \ldots \ldots \ldots \ldots$. . . . . . F F 111

4.5-2 Possible prototypes for facial icons, example $2 \ldots \ldots \ldots \ldots$. . . . . F F 111

4.5-3 Proposed sign for buried radioactive waste . . . . . . . . . . . . F F-113

4.5-4 Proposed Level II message . . . . . . . . . . . . . . . . . . . . . F-115

4.5-5 Example of a map in the Level IV chamber indicating the locations of nuclear waste sites around the world . . . . . . . . . . . F F-116

4.5-6 Diagram for Level IV message indicating much more accurate locations
for the sites shown in Figure $5.4-5 \ldots \ldots \ldots \ldots \ldots \ldots \ldots \ldots \ldots$

4.5-7 A perspective view of the repository for Level III messages, showing waste panels, shafts, marker features, and the reader's present location on the surface (arrow) . . . . . . . . . . . . . . . . . . F-119

4.5-8 Level III diagram that allows the date of burial and the time that has passed since burial to be determined if precession of the pole

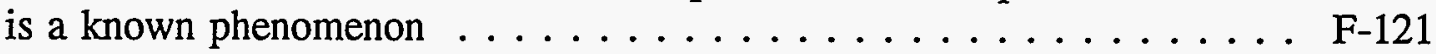

4.5-9 Level IV diagram of the periodic table of elements . . . . . . . . . . . F-122 
4.6-1 Detailed perspective view of the repository and site (for the Level IV

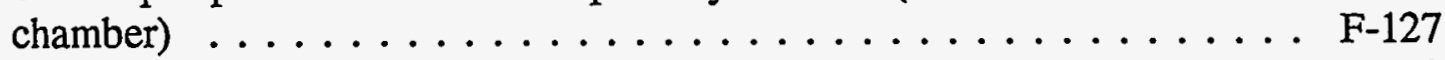

4.6-2 Details of the shaft sealing (for the Level IV chamber) . . . . . . . . F F-128

4.6-3 Detailed geologic cross-section of the site (for the Level IV chamber) . . F F-131 
You are not obliged to finish the task, Nor are you released from undertaking it.

(Ethics of Our Fathers II:21)

(Pirkei Avot II:21)

The land was not willed to you by your ancestors

- it was loaned to you by your children

(Kenya saying)

\section{OZYMANDIAS}

Percy Bysshe Shelley

I met a traveler from an antique land

Who said: Two vast and trunkless legs of stone

Stand in the desert. Near them, on the sand, Half sunk, a shattered visage lies, whose frown, and wrinkled lip, and sneer of cold command, Tell that its sculptor well those passions read Which yet survive, stamped on these lifeless things, The hand that mocked them and the heart that fed; And on the pedestal these words appear: "My name is Ozymandias, King of Kings: Look on my works, ye Mighty, and despair!" Nothing besides remains. Round the decay of that colossal wreck, boundless and bare The lone and level sands stretch far away. 


\section{EXECUTIVE SUMMARY}

Sandia National Laboratories charged a panel of outside experts with the task to design a 10,000-year marking system for the WIPP (Waste Isolation Pilot Plant) site, and estimate the efficacy of the system against various types of intrusion. The goal of the marking system is to deter inadvertent human interference with the site. The panel of experts was divided into two teams. This is the report of the A Team; a multidisciplinary group with an anthropologist (who is at home with different, but contemporary, cultures), an astronomer (who searches for extra-terrestrial intelligence), an archaeologist (who is at home with cultures that differ in both time and space from our own), an environmental designer (who studies how people perceive and react to a landscape and the buildings within them), a linguist (who studies how languages change with time), and a materials scientist (who knows the options available to us for implementing our marking system concepts). The report is a team effort. There is much consensus on the design criteria and necessary components of the marking system. Understandably, there is some diversity of opinion on some matters, and this is evident in the text.

We developed several criteria for the marking system:

- The site must be marked. Aside from a legal requirement, the site will be indelibly imprinted by the human activity associated with waste disposal. We must complete the process by explaining what has been done and why.

- The site must be marked in such a manner that its purpose cannot be mistaken.

- Other nuclear waste disposal sites must be marked in a similar manner within the U.S. and preferably world-wide.

- A marking system must be utilized. By this we mean that components of the marking system relate to one another in such a way that the whole is more than the sum of its parts.

- Redundancy must play a preeminent role in marking system design. The designs considered here have redundancy in terms of message levels, marking system components, materials, and modes of communication.

- Each component of the marking system should be made of material(s) with little intrinsic value. The destructive (or recycling) nature of people will pose a serious threat to the marking system.

- The components of the marking system should be tested during the next few decades while the WIPP is in operation, not only for the longevity of the materials but for the pan-cultural nature of the message. 
In other words, as with the repository design itself, the team was comfortable with the thought of designing a marking system that would last 10,000 years if left undisturbed. Our efforts focused on making it understandable while providing minimal incentive to disturb it. We also consider a public information effort a necessary part of the marking system design. A system that is not understood today has no chance of being understood in the far future.

Figures 4.3-1 through 4.3-18 provide a basic description of our most developed design and other design options ${ }^{1}$ (for these figures and more details please see: Section 4.2, Design options, and Section 4.3, A visual depiction of various design options).

The central area of interest is surrounded by earthen berms. For the WIPP site, the area of interest is where we do not want drilling or excavation to occur. In the design the central area is the area of the underground panels plus either (1) a one-fourth-mile buffer zone, or (2) the distance to which the radionuclides may migrate during the 10,000 -year period, whichever is larger. The forms of the earthworks are jagged and rough, suggestive of energy radiating from the central area.

The berms serve several purposes. First, they define the area of interest. Their size is set so that sand dunes are unlikely to cover all of them entirely at the same time. Instead, the wind will leave dunes streaming behind the berms and create an even larger marker. Second, their shape sets the tone for the entire landscape -- non-natural, ominous, and repulsive. Third, the corner berms are higher than the others and provide vantage points for viewing the entire site. Fourth, the corner berms also include buried rooms with all the message levels recommended for inclusion in this marker system. As the berms erode, these rooms will become uncovered at various times.

The investigator will be guided toward the center of the site by the berms. Prior to entering the central area, however, he or she will encounter a "message kiosk" (Figure 4.3-18). Each message kiosk is composed of a message wall and a protecting wall. In terms of site layout, the message kiosks form the only "nurturing" part of the marking system design. The protecting wall is of concrete and is meant to protect the message wall from erosion. The message wall is of granite or other hard rock and is a vertical, curved form. There are two reasons for a curved form: (1) it makes it very difficult to reuse the piece for another purpose, and (2) it is not an honorific form such as an obelisk. The vertical aspect minimizes tensile stress on the components.

The message wall will bear what we call Level II and Level III messages (cautionary and basic information, respectively). The preliminary texts read:

The body of the report reviews several designs that were considered and rejected. We include them because we believe it is as important to document the decision-making process as it is to present the conclusion. "Menacing Earthworks" is the final recommendation for the overall design option, along with the other marker system components discussed in the text. 
Level II:

\author{
DANGER. \\ POISONOUS RADIOACTIVE WASTE BURIED HERE. \\ DO NOT DIG OR DRILL HERE BEFORE A.D. 12,000.
}

Level III:

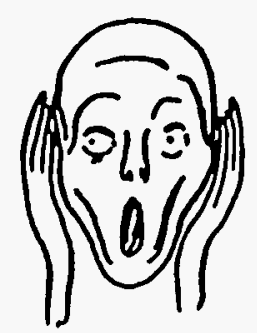

These standing stones mark an area used to bury radioactive wastes. The area is ... by ...

kilometers (or...miles or about...times

the height of an average full grown male person)

and the buried waste is...kilometers

down. This place was chosen to put this dangerous material far away from people. The rock and water in this area may not look, feel, or smell, unusual but may be poisoned by radioactive wastes. When radioactive matter decays, it gives off invisible energy that can destroy or damage people, animals, and plants.

Do. not drill here. Do not dig here. Do not do anything that will change the rocks or water in the area.

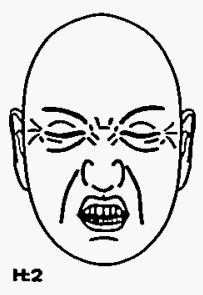

Do not destroy this marker. This marking system has been designed to last 10,000 years. If the marker is difficult to read, add new markers in longer-lasting materials in languages that you speak. For more information go to the building further inside. The site was known as the WIPP (Waste Isolation Pilot Plant) site when it was closed in....

[Face on the right reprinted with permission from: Eibl-Eibesfeldt, Iranäus. Human Ethology. (New York: Aldine de Gruyter) Copyright ${ }^{\circ}$ 1989 by Iranäus Eibl-Eibesfeldt.]

The Level II message is flanked on either side by a face, one denoting horror and the other denoting sickness or nausea. The messages are repeated seven times: the six languages of the United Nations (Arabic, English, Spanish, French, Russian, and Chinese), Navajo, and blank. The blank area is so the message can be inscribed in another language when these grow too ancient to read comfortably. Also included on the message wall is a map of the WIPP site, showing both surface and underground features. This would allow the future investigator to 
Appendix F: Team A Report

reconstruct the site at the time of original construction regardless of what has happened to the site in the interim.

Beyond the message walls lies the central area. A major feature of the design is the concept of a hollow center, that is, nothing at the center is a focal point of interest. Within the central area, but not at the center, lies a "Level IV" room (Figure 4.3-17). ${ }^{2}$ This room has both messages listed above as well as an extended text and additional pictures.

We have constructed two possible texts for the Level IV message. Straight brackets, [ ], enclose comments for this report. The shorter one of the two is:

This place is a burial place for radioactive wastes. We believe this place is not dangerous IF IT IS LEFT ALONE! We are going to tell you what lies underground, why you should not disturb this place, and what may happen if you do. By giving you this information, we want you to protect yourselves and future generations from the dangers of this waste.

The waste is buried ... kilometers down in a salt layer. Salt was chosen because there is very little water in it and cracks caused by digging the rooms for the waste reseal. There is a pocket of pressurized salt water ... $\mathrm{km}$ below the waste. There is a rock layer... kilometers below the surface that did not have drinkable water when we built the site. We studied all the things that could go wrong with the site. We found out that the worst things happen when people disturb the site. For example, drilling or digging through the site could connect the salt water below the radioactive waste with the water above the waste or with the surface. The salt water could wash through the waste and bring the poisonous and radioactive waste to the water near the surface or to the surface itself. People who drink the water will drink the poison. If the water is used for animals or crops, those too will be poisoned and the people who eat them will be poisoned. It may take many years for the sickness and death to show. Radioactivity poisons people because it

${ }^{2}$ designed to be entered and studied in great detail. 
can cause cancer. When radioactive matter decays, the energy it releases can damage the basic material of life in each cell of the human body. The damage can cause uncontrolled cell growth, called cancer, that can kill.

The waste is buried in 845,000 metal drums in a space of about 6,200,000 cubic feet. The waste was generated during the manufacture of nuclear weapons, also called atomic bombs. It is basically laboratory and manufacturing materials that are contaminated with radionuclides having atomic numbers greater than 92, half-lives exceeding 20 years, and concentrations exceeding 100 nanocuries per gram. (A gram of radium is a curie of radioactivity. There are 1,000,000,000 nanocuries per 1 curie. A nanocurie corresponds to the disintegration of 37 atoms in one second). The waste includes metal objects (such as hand tools, machine tools, and motors), glass objects (such as cups and containers), plastic objects (such as bags, tubes, and gloves), and paper and rag materials, such as protective clothing worn by people when they worked with the radioactivity. Many of these materials will corrode, decay or otherwise disappear but the radioactivity will remain.

Pictures on the walls of this room help explain the message. A map shows the surface marking system, its relationship to the underground area used for disposal, and the depth of disposal. A map shows the rock layers below the site. A periodic table identifies those elements that are radioactive and those that are buried below here. When the site was closed in ..., it contained

$$
\begin{array}{ll}
\text { plutonium-239 } & =\ldots \text { curies } \\
\text { plutonium-240 } & =\ldots \text { curies } \\
\text { americium-241 } & =\ldots \text { curies } \\
\text { uranium-233 } & =\ldots \text { curies } \\
\text { thorium-229 } & =\ldots \text { curies } .
\end{array}
$$

Radioactivity declines exponentially with time.

By 10,000 years, after the waste was buried here, the waste will be no more hazardous than the ore 
from which the radioactive material was taken [see $50 \mathrm{FR}$ 38071a].

There is a picture showing the four brightest stars that can be seen from the site (Sirius, Canopus, Arcturus, and Vega). The position of the star-rise changes in time, and lining up the angles of the star-rise with the map will show how much time has passed since the site was closed. The site was closed in

...AD (anno domini), Gregorian calendar

...AD, Byzantine calendar

..., Jewish calendar

..., Islamic calendar

..., Chinese calendar

The waste also contains hazardous materials, whose danger does not lessen with time. These include: lead, cadmium, chromium, barium, methylene chloride, and toluene. The elements also have an arrow in the box in the periodic table. The chemical form for methylene chloride and toluene are shown, also.

If you find unusual sickness in this region, or you find higher than normal levels of radioactivity in the area, inspect the area of the site. Look for: boreholes that were drilled after the site closed, but were never sealed; old mine shafts that were never sealed; and failed seals from the original repository. Reseal these areas, using your best technology, to prevent any further leakage of radioactivity or toxic materials.

Do not destroy these markers. If the message is difficult to read, rewrite the message in your language. in the blank area on this wall. If the markers are worn or missing, add new ones in longer-lasting materials in languages that you speak. This site, built in ... by the United States of America government, represents a first attempt to responsibly dispose of wastes for an extended period of time. Other sites exist that contain radioactive wastes, and they are marked in a similar manner. We have shown these sites on a map in this room. Do not disturb any of these sites. 
These messages will be inscribed onto the walls of the room. Some pictures will be inscribed on stones standing on the floor of the room. (Pictures need be done only once since they do not need to be translated.) The messages will actually be done on two layers, so as one is removed, the message still remains. The configuration of the stones should be such that they can fit together without mortar and can only fit together in one way. The backs of the first layer should be uneven. This will minimize their ease for reuse.

The periodic table will include a sample of each element in the respective box. Naturally radioactive materials will have the radiation trefoil in the box. Man-made radioactive materials occurring in the WIPP will also will have the radiation symbol plus a downward pointing arrow proportional to the amount stored in WIPP. This will allow the investigator to reconstruct the radioactive contents of the WIPP site. To indicate the non-radioactive content of the WIPP, a differently designed, less prominent, downward arrow could be used to indicate the content of other elements. A star map will allow the investigator to estimate the amount of time that has passed since closure has occurred.

The interior of the room is not easily accessible, since the size of the openings makes it difficult to carry away internal parts of the room. There will be five Level IV rooms at the site - - one on the surface and the other four buried within the corner berms. The surface room will be under layers of earth and stone to minimize the effects of daily and seasonal fluctuations of temperature and moisture.

Other components considered for the surface area of the site include a world map, showing the locations of other radioactive waste disposal sites, reuse of the hot cell as a Level IV room (if it can be decontaminated), a visitor's center/memorial, and aeolian structures.

We also considered three sets of subsurface markers. The first group come into effect once intrusion has begun. Their goal is to stop potential intruders, if only for a short while, and to make them reconsider their actions. These include hard crystalline rock at the walls and ceiling of the waste rooms, and Thermit to be ignited by the intrusion process itself.

The second set of subsurface markers provide warnings to potential intruders before damage is done to the repository system. These include salting the site with small markers. These would be lenticular in shape, about 5 inches in diameter, and bear the Level II message. These would be buried throughout the central area, above the caliche level. They are meant to work their way to the surface via erosion or surface excavations. It is a way to remind the potential home builder or farmer that they really do not want to be there. Materials for these markers include fired clay or glass.

The final set of markers would be emplaced in the shafts. This is the most likely area for radionuclide migration. The material would be located about 50 feet down -- i.e., where surface activities are unlikely to affect it but above the region for the various repository seals. Again, disks dispersed in the backfill with Level II messages are appropriate, as are ones with a message saying "You have reached a shaft - Do not dig here." These would tie the activities at the shaft with the warning markers located around the area. 
Appendix F: Team A Report 


\section{General Considerations}

\subsection{Background information on marking the WIPP site}

The Waste Isolation Pilot Plant (WIPP) is a research and development facility for the disposal of defense wastes. Defense wastes are primarily transuranic wastes (TRU). TRU is defined as materials contaminated with isotopes with an atomic number greater than 92 , a half-life greater than 20 years, and a concentration greater than 100 nanocuries per gram. The existence of such a site was mandated by Public Law 96-164 (Department of Energy National Security and Military Applications of Nuclear Energy Authorization Act of 1980).

The WIPP site is located in southeastern New Mexico, about 25 miles east of Carlsbad. The site currently consists of a 16 square-mile area (the land withdrawal area) and a fenced area that is approximately 1.5 miles on a side. Within the secured boundary lie the waste handling building and subsidiary offices. The underground waste disposal panels and rooms are designed to lie within the secured boundary. These panels and rooms are designed to occupy an area that is 2,064 feet by 2,545 feet at a depth of 2,157 feet. There is an overlying aquifer, but the water is not potable. The site is located in an arid region (about 12 inches of rainfall per year) that supports cattle grazing but not dry farming. The arid landscape is undulating in the southeastern part of the site with both stabilized and mobile sand dunes.

WIPP is regulated by an EPA standard set in 1985 [Ref. 1-1].

There are several important features of the Standard:

- It requires a marking system at the site; i.e., it states that "Disposal sites shall be designated by the most permanent markers, records, and other passive institutional controls practicable to indicate the dangers of the wastes and their location" (40 CFR 191.14(c)).

- The performance assessment for the disposal facility must be probabilistically-based. That is, not only must the consequences of a given scenario be calculated, the likelihood of that scenario must be estimated (40 CFR 191.13).

- The time period of interest is 10,000 years (40 CFR 191.13 (a)).

- Active institutional controls are considered effective for no more than 100 years (40 CFR 191.14(a)).

In other words, there is a legal requirement to mark the site. It is this requirement that led Sandia National Laboratories to convene what are known as the Futures panel and the Markers panel. The first group examined the possible "futures" over the next 10,000 years and considered a wide range of conceivable cultures, population sizes, and technical developments. The role of the Markers panel is to develop design characteristics for marking systems for the WIPP site and to judge their effectiveness against the intrusion scenarios developed by the 
Appendix F: Team A Report

Futures panel. The charge to the Markers panel will be discussed in more detail in Section 1.2.

The Sandia work is the second major effort to consider the long-term marking of nuclear waste disposal sites. The U.S. Department of Energy convened the Human Interference Task Force (HITF) in 1980 [Ref. 1-2].

The value of that work lies in establishing the credibility and feasibility of the effort to design long-term marking systems for nuclear waste disposal sites. The Sandia approach differs from the HITF approach in two important features:

- The Sandia approach divided the experts into two teams. The reports, then, reflect interdisciplinary team efforts rather than the focus of individual specialties. It should not be surprising that some designs presented in this report are dramatically different from those presented a decade ago.

- The Sandia approach involves the elicitation of subjective probabilities for the likelihood of deterring human interference with the site. This part of the effort is required to evaluate whether WIPP meets the probabilistic basis of the EPA regulation.

\subsection{Charge to the expert panel}

The Marking System Teams were given a seven-fold charge:

- Recommend markers that should be used to mark the WIPP disposal site.

- Provide physical descriptions of the markers, including size, location, shape, and materials.

- Provide the message on the markers and the method of conveying the messages.

For each major mode of intrusion identified by the Futures panel:

- Estimate the likelihood that each marker has survived (i.e., it is recognizable and the message is apparent).

- Estimate the likelihood that the potential intruder will recognize and correctly interpret the message, given that the marker has survived.

- Estimate the likelihood that a potential intruder will take appropriate action to avoid intrusion given that the marker has survived and that the potential intruder has recognized and correctly interpreted the message.

For the system of markers:

- Re-estimate the likelihood that the system persists, the message is correctly interpreted, and intrusion is deterred. 
The focus of this report is the first three items, which will form a basis for probability estimates from each individual member of the team. Finally, the Markers panel was instructed not to consider cost when developing marking system designs.

The following working assumptions have governed the panel's view of the possible scenarios relating to its charge:

- Climate will vary from that of a desert or near desert to that of good grassland. At best, water will be a scarce resource. Probability of significant change in availability of water over the next 10,000 years is very low.

- The region will be sparsely inhabited under the best of conditions, most likely by keepers of livestock, once natural gas has been taken out of the area over the next few hundred years.

- A tradition directly descended from one or another of the modern technologically, scientifically, and scholarly developed societies will continue through the next 10,000 years, barring catastrophic developments on a scale that makes that impossible.

- Continuity of human existence guarantees that whatever languages are spoken over the next 10,000 years, they will be lineal descendants of one or more languages spoken now, most probably those most widely spoken and written now.

- Because literacy has not ceased to exist since it was first developed some 6,000 years ago, it will not cease to exist over the next 10,000 years, nor the scientific and scholarly traditions based on it, again barring catastrophic developments on a scale that makes that impossible.

In light of these assumptions, the following scenarios have been considered in relation to the problem of marking the WIPP site:

- Human existence has been reduced to what can be supported by a metal-using technology similar to that of early medieval Europe -- use of iron tools, limited literacy, technology capable of deep intrusion at the site if there was extraordinarily high incentive for doing so. Local inhabitants of the site area are most likely to be livestock keepers and small-scale river-bottom farmers. The probability of an intrusion is relatively low. There is little need for a marking system. A marking system that is awesome and scary, as suggested in this report, may invite its being used for religious purposes or as a place of assembly among groups in the area, but is unlikely to invite deep intrusion, especially considering the effort it would require.

- Human existence has continued with regional ups and downs over the world at the present level of technological sophistication, at least, if not a higher one. But the area of the WIPP site has been a marginal one for human habitation and exploitation because of the cycles of climatic changes between desert and grassland. People encountering the site following a period of desertification are likely to be relatively unsophisticated themselves, livestock 
Appendix F: Team A Report

keepers or resource prospectors. If the site is marked by a massive, awesome, and rather scary marking system, word of it is more likely to be disseminated so that it will come to the notice of officials and scholars and scientists of the time. Once they have learned of it, its massive scale will draw scholars and scientists to study it, decipher the messages inscribed there, and thus become acquainted with the nature of the site and what is buried there. In the absence of such study and reacquaintance with what is there, the likelihood of inadvertent intrusion is greater.

- Human existence went through a period of global catastrophe in which it was reduced to illiteracy and something bordering on a Stone Age level of technology, and then redeveloped new patterns of technological sophistication, new literacy, and new science. The probability that people would then be able to decipher and understand the true meaning of the messages inscribed there is low, unless the inscriptions provide a key to their interpretation. By having the same messages in different languages arranged in a way that shows them to be parallel messages, the site design can provide the equivalent of the Rosetta Stone that will increase the probability of successful decipherment.

It is to the last two scenarios above that our team has considered a marking system to be most relevant. With these scenarios in mind, we decided on a systems approach to marking with

- Several components within a given design,

- Multiple items within each component, and

- Two-way indexing linking different levels of information and system marking components.

With this approach, we can afford to lose items within a given component without seriously compromising the effectiveness of the entire design. (For example, about one-third of the stones of Stonehenge are missing, yet the entire design can be reconstructed without major controversy.) Under these conditions, it is the probability estimate for the entire system that is relevant, not those for individual markers.

Second, a literal interpretation of the charge leads to the estimation of 54 probabilities for each system design ( 2 modes of intrusion $\times 3$ time periods $\times 3$ degrees of efficacy [the marker survives/is understood/and deters] $\times 3$ types of societies [more advanced/similar to our own/less advanced]). Given that we have explored 5 designs, a literal interpretation of the charge leads to several hundred probability estimates. Extending this effort to individual components of a system would further extend the number of needed estimates. Using Occam's razor to slice through this forest of logic branches, the A Team interpreted the work of the Futures panel as the need to be ready for anything regarding marking system design for the WIPP site.

Third, we considered one set of branches to be outside our purview. The regulatory requirement is to deter inadvertent intrusion, and thus we feel that if the message is understood, our job is 
completed. Any action that takes place after the message is understood is advertent and intentional. If the intruder is aware of what lies below him or her, and of the consequences of disturbing the area, and yet does not change his or her intended course of action, it is not inadvertent intrusion.

\subsection{Should the site be marked?}

\subsubsection{Motivations for Marking}

There are two major motivations for marking the WIPP site:

- Social responsibility to the future generations that did not create the waste.

- We have no alternative; the site is already marked.

We therefore feel that it is essential that the WIPP site be marked in some manner, and cannot agree with the conclusions of two of the Futures panel teams and other authors [Ref. 1-3], which suggested not marking it. We take it as uncontroversial that all people have an inherent right to understand as far as possible the forces that might profoundly affect their well-being. We do not accept the reasoning that led to the suggestion not to mark the site. In this view, marking might be counterproductive; given the (presumed) small risk of inadvertent exposure, marking would lead only to the attraction of "curiosity seekers," thereby increasing overall risk. But we are not sufficiently confident that the risk of inadvertent exposure is low and, even if it is, not warning future generations of a potential peril under their feet represents an abdication of moral responsibility.

An analogy seems appropriate here: Inhalation of radionuclides projected to be confined in drums in the Salado formation may well present a greater health hazard than a lifetime of cigarette smoking, and yet our society places health warnings on every cigarette pack.

The performance assessments at the WIPP site indicate that the expected behavior of the site indicates little danger to humans, except for human interference. Although the regulation is probabalistically-based, the team decided to design the site as if it posed the maximum plausible danger. We examined two causes for greater potential danger. First, as one of the Futures panel teams noted, the site may be used to store the more dangerous high-level waste, despite the absence of explicit official plans to use it for this purpose. We can imagine a scenario of a WIPP already in operation, political pressure in other states to ship out-of-state all their radioactive wastes, and a decision not to build the facility at Yucca Mountain, NV, as the repository for the country's high-level civilian and defense waste. ${ }^{1}$ Thus an atmosphere would arise conducive to

1 Section 12 of the WIPP Land Withdrawal Act (WIPP LWA) (Public Law 102-579), approved October 30, 1992, entitled Ban on High-Level Radioactive Waste and Spent Nuclear Fuel, states:

The Secretary shall not transport high-level radioactive waste or spent nuclear fuel to WIPP or emplace or dispose of such waste or fuel at WIPP.

While Congress has spoken on this issue, Team A found it conceivable that the WIPP LWA would be amended 
concentrating the nation's high-level radioactive refuse, whatever its ultimate source, at the WIPP. There is even some support in the State of New Mexico for this plan: in 1987 the governor suggested that the WIPP site should serve as a repository for high-level commercial waste. Moreover, the remote handling area of the WIPP building could be used to handle high-level wastes without redesign, and the site could be expanded either laterally or at a deeper layer to accommodate the additional wastes.

Second, whatever wastes are ultimately stored at the WIPP, there is a probability significantly greater than zero that they are not as secure in the Salado salt beds as might be hoped. The Scientists' Review Panel on WIPP [Ref. 1-4] has warned that brine seepage in the beds will in all probability lead to corrosion of the canisters. This contaminated water could find its way into the Rustler Aquifer (which feeds the Pecos River and is located only around 1000 feet below ground level) through the access shafts filled with disturbed salt or through boreholes created by drilling. ${ }^{2}$

Even if this is only a very remote possibility (it is, indeed, one which we lack the technical expertise to evaluate), the potential danger provides a powerful argument for marking the site.

In a real sense, there is little point in pressing further the argument that the site should be marked for the simple reason that it already is marked (or will be if it is ever operational). So much buried metal and radioactive material will leave a "signature" that scientists of the future will have no difficulty in detecting. What we need to do, of course, is to "complete" the marking by letting them know why it is there. Also, it is projected that after settling of the excavated and filled salt deposits, ground levels will be depressed by at least a half foot. Even today's geologists and archaeologists can detect such a depression; those of the future will presumably be able to do so even more readily.

It must be noted that marking the site is incompatible with the recommendation that after the last drum is buried the site be restored to a pristine condition. We are sympathetic to environmentalist concerns that WIPP leave no permanent trace on the landscape, but we feel that in this case health and safety requirements outweigh aesthetic ones.

\subsubsection{General Criteria for any Marking System}

Any system for marking the WIPP site will have to be colossal in scale. Given the many huge human-made structures in the world today and the many more that are likely to be built in the coming centuries, a marker consisting of a small building or sculpture bearing a standard commemorative plaque is unlikely to be effective. Many of these existing structures are in cities, but others are in remote areas and thus potentially compete for attention with anything marking the WIPP site. In the U.S. alone, there are dozens of National Battlefields, National Historical Parks, National Memorials, and so on, most (like the WIPP) away from major

to allow other types of waste at the WIPP. They thus considered all scenarios, even those with a low probability. Marker text will be finalized to reflect the contents at closure.

${ }^{2}$ For related information from the SAND92-1382 authors, see p. F-153. 
conurbations and each containing statuary and commemorative markers. In order to avoid the risk of the WIPP markers being confused with them and ignored (who in the 72nd century is going to bother to have a dedication to some 19th century war hero decoded?), they and their connecting structures have to be conceived of on a scale equivalent to that of the pyramid complexes of Egypt.

Put simply, the marking system must be on a sufficiently grand scale to provide future generations with the motivation for going to the trouble to translate the message on the markers. We have no doubt that, barring a global cataclysm that results in a pre-technological culture, there will always be scholars in the world capable of translating the major languages of the twentieth century. The question we must ask with respect to the markers is: Why should they bother to do so? Inscriptions in ancient languages like Hittite, Lydian, Numidian, and so on are readily translated for the simple reason that there are so few of them. But thousands of books are now published each year on an acid-free paper that promises to survive the centuries. More to the point, the world today is filled with durable structures, of which monuments are only one type, most of which are marked with inscriptions of some sort. In short, because it is highly likely that much written material from our culture will survive long into the future, no intruder into the WIPP site will have the slightest interest in going to the (perhaps considerable) trouble of having its markers translated unless he or she can be convinced that the importance of the site would make not doing so perilous.

\subsection{International aspects of marking}

This panel is only the second to attempt a coherent design of a marker system for radioactive wastes, and it is important that we think on a more encompassing scale than just for the WIPP site.

The previous panel, called the Human Interference Task Force, was convened for DOE by Battelle's Office of Nuclear Waste Isolation. See their 1984 report: BMI/ONWI-537. Reducing the Likelihood of Future Human Activities That Could Affect Geologic High-Level Waste Repositories [Ref. 1-2].

The disposal of radioactive wastes is an international problem, and although present political boundaries shape many aspects of how the problem is being defined and handled today, it is clear that these boundaries have absolutely no relevance to the generations of future millennia. It is therefore essential that any WIPP markers be designed as part of a global system of marked sites. Figure 1.4-1 gives a rough idea of how long-term disposal sites are likely to be scattered around the world; by various measures the U.S. represents only one-sixth to one-third of the total (for instance, about one-quarter of the world's nuclear power plants are in the U.S.) [Ref. 1-5].

We urge that an international standard be developed for the marking of long-term disposal sites. A degree of commonality between sites all over the globe provides a redundancy that should greatly enhance the likelihood of any given site's markers working to deter intrusion. Even if the markers at a given site become misinterpreted or baffling, their similarity to those at other sites where the message is understood will provide a means for the message to be reinstated. 

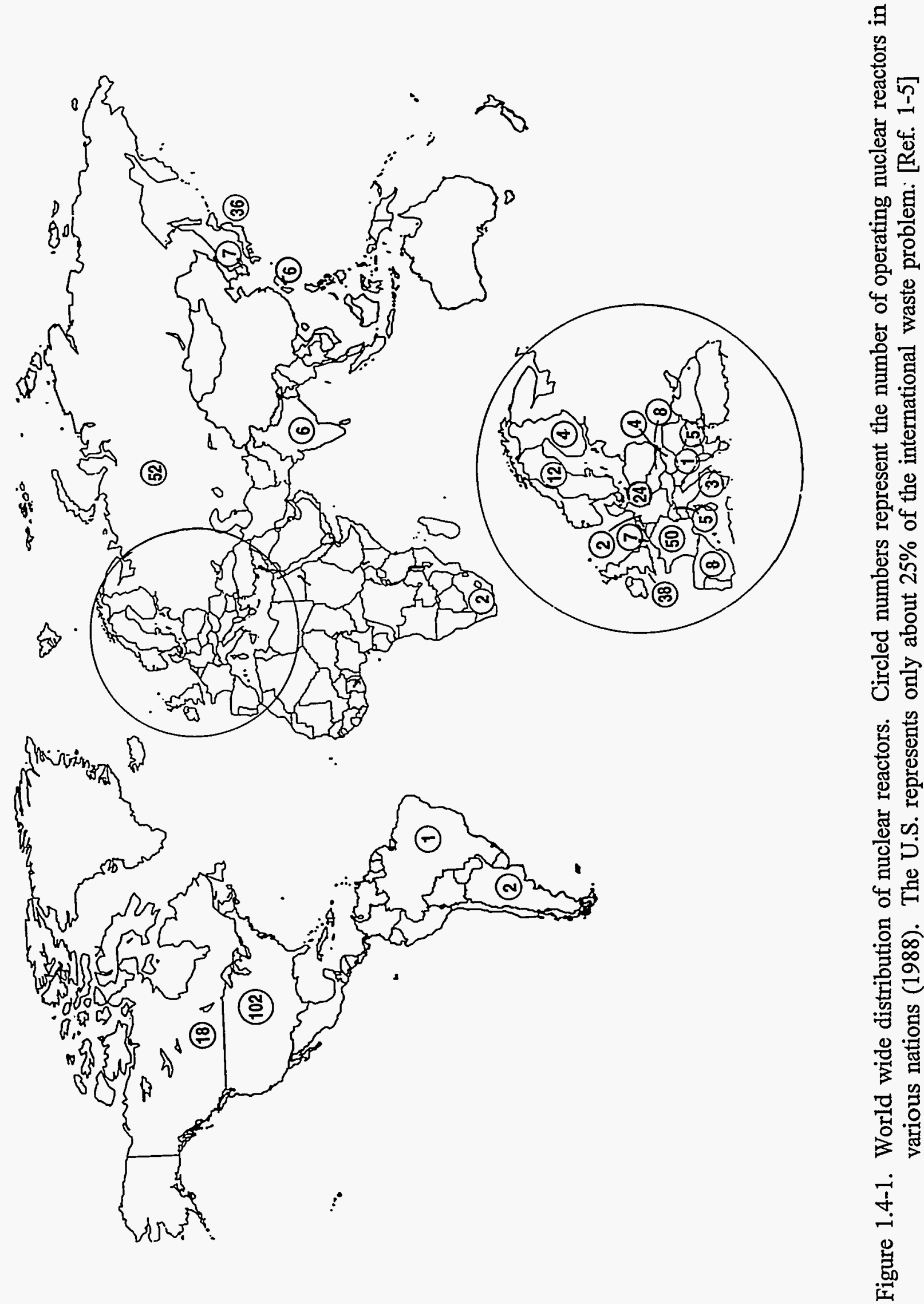
Furthermore, if each site refers in some way to the specific locations of all other sites (as we propose in Section 4.5.4), then it will be possible to reinstate the message even if a site's marker system has physically disappeared due to natural catastrophe or deliberate destruction.

The international standard should not dictate the details of design and construction for the entire marker system. It would be both politically unrealistic and foolish from an engineering and cultural point of view to try to do so. Instead, the standard must give a few basic design features to which all marker systems must conform; each individual system will then undoubtedly have many more components. Here is the type of standard that we envision:

Each site must:

(1) display its basic warning message

[what we call Level II in this report, 10-15 words]

in at least the following languages:

Chinese, Russian, English, Spanish, French, and

Arabic [the UN languages] and the local

language in common use if not otherwise listed;

(2) prominently display the international

radiation symbol flanked by horror faces;

(3) display in a protected chamber a world map of

all disposal sites, together with a standard

diagram [Fig. 4.5-6, and Section 4.5.4] that

geometrically allows their location to an

accuracy of at least $5 \mathrm{~km}$; and

(4) include earthen berms to delineate the

disposal area with heights of at least $10 \mathrm{~m}$.

This last standard is only an example, the important aspect of it is that there be some common aspect to all sites that is large-scale, long-enduring, and not dependent on languages or graphics.

\subsection{A systems approach...two major themes}

This team's thinking is founded on two major themes. The first theme states that the use of communication technology cannot bypass the problem of the certain transformation and succession of cultures, but use of fundamental and enduring psychology can. The second theme states that the entire site must be experienced as an integrated system of mutually reinforcing messages, and designed accordingly. These themes are discussed below. 
A system for bypassing the vagaries of cultural transformation: Most general models of communications assume that sender and receiver co-exist in time, are to some extent known to each other, and share a culture sufficiently similar to reduce cross-cultural noise.

In this project we face the unique problem of a sender and receiver living in epochs so enormously time-distant from each other that we know little of what the political, economic, symbolic, linguistic, social, and technological realms of probable future cultures will be like. Further, we assume a succession of many such transformed cultures. As a result, much of the past thinking on marking the site has focussed on the problems of cultural phenomena, and on the probability of these phenomena enduring and being useful, especially the technology of structures and materials, and the technology of communications, language, pictures, and symbols. But precisely because they are cultural phenomena, they too will have an historically predicted rapid rate and range of transformation, which makes most culture-related prognostication uncomfortably speculative. Past assumptions regarding markers posit that this discomfort could be reduced through better technologies. We strongly recommend an alternative strategy, and have adopted it as a theme in our work.

This team's fundamental premise is to cancel the time-borne cultural "distance" between sender and receiver by concentrating on fundamental and enduring phenomena shared by all humans, things that are species-wide now, probably always have been, and will continue to be, phenomena, that is, that bypass culture(s), and have enormous endurance. Only such phenomena can render moot the transformation of cultures. Such phenomena are "archetypal," called so because they were already meaningful before the emergence of language and culture in human evolution and because they are universal to human existence even with language and cultural differences, and therefore, all cultures use them as their common basic material, transforming them into each culture's specific ways, what Joseph Campbell calls "ethnic variations." (Givens [Ref. 1-6] cites many of these.)

The stuff of both our messages and our mode of communication is the fundamental psychic structure of humans, their world-wide predilection for symbol formation, and the bonding of meaning to form in species-wide archetypes.

This focus on archetypal forms-bonded-to-meaning assures survivability of content against all events and processes that leave our species biologically unchanged. It focuses on meaning and feeling content that is already in the mind and body before language, and thus is not dependent on it. (In this report, the most extensive explication of what archetypes are, their origins and behaviors are in Sections 3.2 and 5.4.)

The entire site as a system of communication: If archetypal meanings are to be transmitted, and because these meanings originated during hundreds of thousands of years of our activity in an experientially whole environment, they should be best communicated in and through an experientially whole environment. Thus, our medium of communication is the entire environment experienced near and at the WIPP site.

This mode of experientially-whole environment-based communication cannot be achieved by standing stone markers on an otherwise unchanged site...in fact, such designs may be easily 
misinterpreted. We choose to focus on the conscious design of the human experience of the entire area and all its subelements, which is both the mode and the content of communication, where meanings are bonded to and embodied in form.

We intend that all our physical interventions at the site serve as parts of a communications system and that all elements of this system carry archetypal symbolic content...from the layout of the entire site down to the location and shape of thermal expansion joints.

As well, we use the more culture-bound modes of communications such as languages and diagrams, but these are used as part of a larger system of communications. This system is to be one with great redundancy of messages and modes, so that even with some loss the goals of the system are met.

As well as being conceived as (1) a whole communication experience, (2) having a systemic character in which pieces are related in meaningful ways that add meaning, and (3) being sufficiently redundant to endure loss of elements, we apply the principle of Gestalt, in which the experience of the total communicated message is greater than the sum of its parts (even with some parts missing or degraded).

Detailed guidelines for design of the site and its subelements so that they achieve these goals are in Section 4 of this report.

\subsection{On-site testing of markers}

The problem of designing a system that will work for all imaginable societies over a period of 10,000 years is daunting. The fact that humans have designed and built systems that have already survived for 5,000 years, however, allows us to believe that this is a feasible and credible task. We also have the advantage that, as planned, the WIPP will not be sealed for at least another 30 years. Although it is less than $1 \%$ of the design lifetime for the marker system, 30 years provide an important opportunity for testing. We strongly urge that a long-term program for testing materials, structures, messages, and concepts be initiated as soon as possible.

The most obvious tests concern the longevity of structures (earthworks, monoliths, rooms), materials (concrete, stone) and the longevity of engravings as they would be placed variously at the WIPP site in the proposed marker system, e.g., 100 feet above the ground, at the surface (with and without various types of protection from the elements) and underground.

A second class of tests is no less important: how well do our basic messages come through for a wide variety of people and cultures? This panel is very unrepresentative of even U.S. citizens. We are all white, highly educated, with only one female, one immigrant, two religious traditions, and a 30-year age range between the participants. The overall site design and the specific messages should be tested for efficacy on a wide variety of persons in the United States 
(various racial and ethnic groups, educational levels, etc.) and in other countries (including undeveloped societies).

Another basic test becomes possible because of the long lead time before the final design of the WIPP marker system. The final marker-design panel (in AD 2030?) can look back at the present panel's recommendations and gauge how ideas have evolved over 40 years. Stability and consistency in the concepts for the major design elements would give them more confidence that they have lasting value; disagreements in approach should cause some hard thinking about the likely success of the markers.

\subsection{References}

[1-1] (EPA) U.S. Environmental Protection Agency. 1985. "Environmental Standards for the Management and Disposal of Spent Nuclear Fuel, High-Level and Transuranic Radioactive Wastes," Federal Register. Vol. 50, no. 182, 38066-38089. The United States Court of Appeals for the First Circuit remanded Subpart B (which prescribes the performance of the disposal facility after closure), but the Department of Energy has agreed to abide by the Standard, as promulgated, until a new Standard is finalized, see "Second Modification to the July 1, 1981, Agreement for Consultation and Cooperation on WIPP by the State of New Mexico and the U.S. Department of Energy."'

[1-2] The main report of the Human Interference Task Force was: Office of Nuclear Waste Isolation. 1984. Reducing the Likelihood of Future Human Activities That Could Affect Geologic High-Level Waste Repositories. BMI/ONWI-537. Columbus, OH: Office of Nuclear Waste Isolation, Battelle Memorial Institute. More detailed individual reports were: Berry, W.E. 1983. Durability of Marker Materials for Nuclear Waste Isolation Sites. ONWI-474. Columbus, OH: Office of Nuclear Waste Isolation, Battelle Memorial Institute; Givens, D.B. 1982. "From Here to Eternity: Communicating with the Distant Future," Et cetera. Vol. 39, no. 2, 159-179; Kaplan, M.F. 1982. Archaeological Data as a Basis for Repository Marker Design. ONWI-354. Columbus, OH: Office of Nuclear Waste Isolation, Battelle Project Management Division; Sebeok, T.A. 1984. Communication Measures to Bridge Ten Millennia. BMI/ONWI-532. Columbus, $\mathrm{OH}$ : Office of Nuclear Waste Isolation, Battelle Memorial Institute; Tannenbaum, P.H. 1984. Communication Across 300 Generations: Deterring Human Interference With Waste Deposit Sites. BMI/ONWI-535. Columbus, OH: Office of Nuclear Waste Isolation, Battelle Memorial Institute; Weitzberg, A. 1982, Building on Existing Institutions to Perpetuate Knowledge of Waste Repositories. ONWI-379. Columbus, $\mathrm{OH}$ : Office of Nuclear Waste Isolation, Battelle Memorial Institute.

[1-3] Rochlin, G.I. 1977. "Nuclear Waste Disposal -- Two Social Criteria," Science. Vol. 195, no. 4273, 23-31.

[1-4] Rosenthal, D. 1990. At the Heart of the Bomb: The Dangerous Allure of Weapons Work. Reading, MA: Addison-Wesley. 
[1-5] American Nuclear Society. 1988. "World List of Nuclear Power Plants Operable, Under Construction, or on Order (30 MWe and Over) as of December 31, 1987," Nuclear News. Vol. 31, no. 2, 63-82.

[1-6] Givens, D.B. 1982. "From Here to Eternity: Communicating with the Distant Future," Et cetera. Vol. 39, no. 2, 159-179. 
Appendix F: Team A Report 


\section{The Problem of Message}

\subsection{Message definition}

Modern understanding of the communications enterprise shows that there can be little separation of the content of a message from its form, and from its transportation vehicle. They affect each other, and all of it is message. McLuhan and Fiore [Ref. 2-1] take that even further, arguing that "the medium is the message." Given this, rather than our attempting to first articulate messages, then to select their form, and then to design their vehicle, we choose to do as much of this simultaneously as is reasonable, attempting to accomplish

- a Gestalt, in which more is received than sent,

- a Systems Approach, where the various elements of the communication system are linked to each other, act as indexes to each other, are co-presented and reciprocally reinforcing, and

- Redundancy, where some elements of the system can be degraded or lost without substantial damage to the system's capacity to communicate.

Everything on this site is conceived of as part of the message communication...from the very size of the whole site-marking down to the design of protected inscribed reading walls and the shapes of materials and their joints. In this report, the various levels of message content are described, as is the content of each level, the various modes of message delivery, and the most appropriate physical form for each.

We obviously recommend that a very large investment be made in the overall framework of this system, in the marking of the entire site, and in a communication mode that is non-linguistic, not rooted in any particular culture, and thus not affected by the expected certain transformation of cultures. This mode uses species-wide archetypes... of meanings bonded to form, such that the physical form of the site and its constructions are both message content and mode of communication. Thus, the most emphatically delivered message is the meaning-bonded-to-form in the site itself. (See Section 4 for the message the site is asked to deliver.)

As part of a system of message communication, we recommend substantial use of verbal texts and graphics, but with little emphasis on constructed, non-natural, non-iconic symbols. These texts and graphics act as indexes to each other, and act as indexes across message levels. We also suggest the site be marked so it is anomalous to its surroundings in its physical properties such as electrical conductivity and magnetism. 
Appendix F: Team A Report

\subsection{Message levels and criteria}

\subsubsection{Message Levels}

Givens [Ref. 2-2] describes four information levels for the messages:

- Level I: Rudimentary Information: "Something man-made is here"

- Level II: Cautionary Information: "Something man-made is here and it is dangerous"

- Level III: Basic Information: Tells what, why, when, where, who, and how (in terms of information relay, not how the site was constructed)

- Level IV: Complex Information: Highly detailed, written records, tables, figures, graphs, maps, and diagrams

Our discussions led to two expansions of Givens' work. First, we decided that it was possible to convey a sense of danger, foreboding, and dread without the use of language or pictures. This would be done within the context of site design. Under these circumstances, what would generally be considered as Level I components (e.g., earthworks) would be able to convey both Level I and Level II messages. Second, we decided to have a fifth level that lay between Givens' Level III and Level IV. The new Level IV would have more detail than Level III but still not be the complete rulemaking record. The latter level is now called Level V. Specific examples of the different level messages are given in Section 4.6.2.

The general approach taken by the team is that the emphasis is on clarity and, where possible, brevity. Overly long and complex messages will be too difficult and time-consuming to translate to be effective. The message must be straightforward and neither understate nor overstate the hazards of the site. The difficulty in formulating the message is that many normal human activities, e.g., house building and farming, can occur on the surface without jeopardizing the performance of the repository. Problems begin only when deeper drilling and excavation occur.

We decided against a large radiation symbol prominently displayed on a marker lest the potential intruders take a quick reading, find nothing more than background radiation, and ignore the rest of the message. We did decide that the incorporation of a radiation symbol was appropriate within the larger context of the message. As a symbol, it could provide a link between textual and pictorial information.

We decided against simple "Keep Out" messages with scary faces. Museums and private collections abound with such guardian figures removed from burial sites. These earlier warning messages did not work because the intruder knew that the burial goods were valuable. We did decide to include faces portraying horror and sickness (see Sections 3.3 and 4.5.1). Such faces would relate to the potential intruder wishing to protect himself or herself, rather than to protecting a valued resource from thievery. 
We decided against overstatement of the danger. The "Touch one stone and you will die" approach is unacceptable because it is not credible. Inevitably, someone will investigate the site in a non-intrusive manner. Nothing will happen to that person, and the rest of the message will therefore be ignored. There was consensus, however, on the need to mark the site and on the need to convey the dangers to the potential intruder.

We consider the key to a successful marking system to be a credible conveyance of the dangers of disturbing the repository. We must inform potential intruders what lies below and the consequences of disturbing the waste. If they decide that the value of the metal component of the waste far outweighs the risks of recovering the metal, the decision is their responsibility, not ours.

The warning information is divided up into multiple message levels and occurs in different spatial configurations to prevent information overload. The Level II message is short and simple. It is meant to function during the time the language is still readable by the intentional intruder. If a sufficient amount of time has passed that the language is difficult to interpret or needs to be translated, the Level III and Level IV messages provide larger blocks of text that will be easier to translate.

The general guideline for the message levels is that they are linked or indexed. Any intruder that can comprehend a given message level will be able to comprehend lower message levels. At least two levels of information appear on or in any given component of the marking system, thus allowing a link from lower to higher level messages. If there is not a physical link between message levels on a given component, there is a linguistic "pointer" that there is another set of information at the site.

\subsubsection{Criteria}

Givens [Ref. 2-2] presents criteria for a warning system for a nuclear waste disposal site. We have addressed the criteria in our designs. The designs presented here use a mixture of iconic, symbolic, and linguistic signs.

Iconic signs are used with written languages to convey information for message Levels II through IV. Unlike Givens, the team had difficulty designing an iconic narrative that could unambiguously convey complex information, such as contamination of the food chain and its effects on human health. As he points out, a picture may be worth a thousand words, but it may be difficult to determine which thousand words a set of pictures may evoke. We will be interested to learn of B Team's work in this area.

We in Team A, however, selected sample icons for use within the marker system. They are limited in number, have emotional impact, and are not culturally bound. Section 4.5 gives examples of the potential icons to be used within the marking system. 
Symbols do not play a large role in our marking system. The consensus within the team is that symbols are culturally learned. For example, to know that a picture of a beetle means more than a beetle when it appears on an ancient Egyptian tomb wall means that the viewer must be aware that it was a symbol of rebirth. The dung beetle (scarab) rolls around its seemingly lifeless ball of dirt only to have life burst forth from it. This became a metaphor for the beetle rolling the ball of the sun (which gives life) across the sky. The sun disappears (dies) every evening and is reborn every morning. Yet the significance of the scarab could be reconstructed because of its context within language. In a similar manner, the marking system design incorporates the radiation symbol, which has already been established as an international symbol for 40 years, in multiple contexts to allow future readers to reconstruct its meaning.

We found that redundancy in many forms was crucial to the functioning of the marking system. Both textual and non-textual (landscape architecture) methods are used to convey information about the WIPP site. Symbols, icons, and language are used within the textual methods of conveying information. Different languages are used as a means of redundancy within the last category. Another form of redundancy is standardization of a general marking system design and its use at all potentially hazardous radioactive waste disposal sites. This repetition enhances the understandability of the message. WIPP should not be unique. An archaeologist prays to find that unique site or object that will make her or his reputation. Then, when it is found, she or he bemoans the fact that there are no comparisons that can be made to enhance our understanding of the find.

Finally, the site will be marked even if we do not place a marking system there (see Section 1.3). The visual (e.g., surface depression) and non-visual anomalies (e.g., seismic profile) at the site will attract further investigation. Our task is to give the potential intruder sufficient credible information to allow him or her to decide whether to leave the site alone. (Informed, intentional intrusion is not covered by the regulation.) To this end we use a mixture of durable signs and sign vehicles to claim the area boldly as one set aside for a specific and special purpose.

\subsection{Which message level is necessary to deter intrusion?}

\subsubsection{Activities Near the Site}

At the onset of this task, the Markers panel received an introduction to the WIPP site and background information on the research to date. The introduction included a review of scenarios developed by the Futures panel teams and the possible modes of intrusion by both near-site and on-site activities. Follow-up information included performance assessments for several scenarios involving intrusion by exploratory boreholes for hydrocarbons. The team considered subsidiary markers at nearby towns to link with the marking system at the WIPP site itself. We decided against this approach because (1) it was too easy to misinterpret the subsidiary marker as indicating another smaller repository, and (2) it was too difficult to identify all the potential areas where such activities would occur during the next 10,000 years. We believe that it is appropri- 
ate to place written information at nearby towns to inform the local population about the site and the danger of activities that could affect its performance. There is a general request, however, in the Level III message not to disturb the rocks or water at the site. This is a link between the marking system at the site itself and off-site activities, and is consistent with the charge to the Markers panel for an emphasis on preventing boreholes at and excavation of the site itself.

\subsubsection{Activities at the Site}

A Level I message without cautionary intent or higher level messages is insufficient to deter intrusion. In fact, its presence will simply spur investigation. Therefore an earthwork without cautionary content in its form or without associated higher level messages is not acceptable.

The consensus of the group is that message Levels I through IV should be present at the site itself. Each message level will be repeated more than once in the marking system design for the sake of redundancy.

Level V information, by its very nature and volume, is not suited to engraving on stone. It is suited to the media of acid-free paper, microform, and electronic form (e.g., CD-ROM). These can be reproduced relatively cheaply and dispersed into numerous libraries world-wide. (See Weitzberg [Ref. 2-3] for more details on the dissemination of Level $\mathrm{V}$ information.)

\subsection{References}

[2-1] McLuhan, M., and Q. Fiore. 1967. The Medium is the Massage (sic), New York, NY: Random House, Inc.

[2-2] Givens, D.B. 1982. "From Here to Eternity: Communicating with the Distant Future," Et cetera. Vol. 39, no. 2, 159-179.

[2-3] Weitzberg, A. 1982. Building on Existing Institutions to Perpetuate Knowledge of Waste Repositories. ONWI-379. Columbus, OH: Office of Nuclear Waste Isolation, Battelle Memorial Institute. 
Appendix F: Team A Report 


\section{Components of a Marking System}

\subsection{Communications: site and structures}

MODES OF COMMUNICATION:

Section 3 presents the general background to the modes of communication used to convey the messages. Detailed examples implementing these thoughts are represented in Section 4.

\subsection{General discussion: for the site as a whole and individual site structures}

A major premise of our work is that the physical form of the entire WIPP and each and all the structures on it can itself be a communication...through a universal, "natural language" of forms.

Furthermore, a major component of the site's communicative capacity is the importance we give it. (One measure of importance is the sheer enormity of work done to mark it.) This communication of importance cannot be achieved just through markers on the site. (The use of vertical stone markers not only will not suffice, it well may introduce substantial message ambiguity through their form alone. This is discussed later.)

The capacity to communicate meaning through physical form is based on an enduring human propensity to experience common and stable meanings in the physical forms of things, including the design of landscapes and built-places. Such communication operates in a different mode from, and independently of, linguistic modes of communication. There is an emerging literature on the "semantics of design" in architecture, landscape architecture, and industrial and product design, some of it in our citations.

While some form-carried meanings are certainly based in or modified by cultures, others far more basic both predate and thus transcend (or bypass) particular cultures, forming a species-wide "natural language" we are all either born knowing or learn from the early life experiences that are common to human existence everywhere. These meanings-embodied-inform and communicated through form are archetypal, seem to vary little across cultures or epochs, have already endured with us for over several hundred thousand years, and are expected to endure unchanged for far longer than this project's time frame of 10,000 years.

There are particular places (built-forms and natural and made-landscapes) that elicit powerful feelings in almost everybody. These places feel "charged," almost in an electric sense, and the places seem filled with meaning. Most places, of course, are not charged and few are filled with meaning. The places that do carry charge and meaning are sometimes beautiful, but at least as many are ugly, awesome, or forbidding. Their importance is in their content (the message), far more than their form, and the success of their forms is in their expressive capacity, not their aesthetics. 
These meanings and feelings often come to people in places that are not even of their culture or time. Obvious examples are the way Stonehenge and the painted caves [Ref. 3-1] of Altamira [Ref. 3-2] and Lascaux [Ref. 3-3] evoke profound feelings in modern viewers. This stable and common response to certain places thus seems to transcend particular cultures and particular times. (Recent cross-cultural research in peoples' preferences for types of landscapes supports this.) It suggests an origin in something much broader than individual experience and older and deeper than culture, something that is species-wide, part of what it is to be human.

\subsubsection{The Concept of Archetypes}

Why do the meanings attributed to and feelings evoked by certain types of forms recur so frequently across cultures and epochs? A general answer is offered by work in such fields as cultural anthropology, philosophy, evolutionary biology, semiotics, psycho-analytic theory, mythology, and comparative religion, which suggest that such a phenomenon is explainable by the presence of what some call "archetypes" in us. Archetypes result from inherited propensities to respond to certain forms, or to experience certain forms, in specific ways affectively. Archetypal forms are those that evoke these responsive propensities. Archetypes have always played an essential role in human physiological, social and spiritual functioning, evoking feelings of anger, aspiration, nurturing, desire, community, order, and death, to name some of the phenomena about which we still feel, think, and ponder most profoundly.

Many argue that the origins of our strong feelings and meanings in these special places come from their resonance with something already inside us, like templates in the mind, which have been called various names: Archetype; Imprint, Innate Releasing Mechanism, Primary Image; Elementary Idea, Inherited Memory, Isomorph, Cosmic Model, Embodied Myth, Shadows, Memory Deposit, Engram, and others. An archetype seems to be a naturally occurring creation of human experience and human spirit, but not one fully explainable or explorable through analytic modes of thought. We need not subscribe to theories of a "collective unconscious" or to other explanations for archetypes in order to work effectively with such forms, as artists and architects have been doing for centuries.

\subsubsection{Archetypes Operating as a Natural Language}

If the physical forms of places can communicate meanings, then places have a narrative capacity, a capacity to tell us a story about ourselves. But like each of the symbolic forms (language, dance, sculpture, myth, etc.) engaged in narrative, or re-presentation, form of place tells certain stories well and certain ones less well, depending on the "fit" between each symbolic form's fundamental qualities and mechanisms and the stories it tries to tell. The best voice of place, its most robust and effortless speaking, is through a natural language of spatial physicality. The language is called "natural" because it is a language we do not have to learn...we seem to understand it without learning it.

This is not a symbolic language that one must learn (through one's culture), like the meaning of the cross, the swastika, the trefoil radiation symbol, or that buildings done in Greek or Roman styles today are somehow "more important." 
Meaning is received by all the senses (including the haptic sense of body structure and postures), by the mind, and is probably more felt than understood. It does not have precise meanings, but rather, flickers of, bundles of, even a mosaic of meanings. No absolutely direct translation into language is possible, or even appropriate. Places speak in another way.

As one example of the meanings inherent in a form, let us examine a particular form of vertical stone marker, variously called stele, obelisk, standing-stone, and memorial column. These have been historically and commonly used to commemorate honored phenomena. So, when a people wish to remember an important relationship, event or personage, a location is dedicated to it and often marked with an enduring and aspiring vertical form or sets of them. In natural language, a vertical stone means: an aspiring connector between us (on earth) and an ideal (up there); that we "stand up" with pride about this honored phenomenon. The marker is a symbolic inhabitation of the place it occupies. Its size and workmanship is a sacrifice of much work and resources to a memory. It is a strong suggestion (because we left it to them and it is of durable material) that future people also give honor to the memorialized phenomenon. When we use this particular physical form of the vertical marker, both its historic use as an honorific and its meanings in natural language may well send a message that this is an honored place, a place about a "good" both in our culture and in the culture of future observers. Such a message would be inappropriate for the WIPP site. This discussion is not meant to discard use of markers, but to re-examine some underlying assumptions and, perhaps, to place markers in the context of a larger set of markings. The team recommends the use of vertical masonry markers, if their form feels dangerous, more like jagged teeth and thorns than ideals embodied.

In any scenario(s) for the future, a natural language, one that is relatively independent of cultural conditioning and can survive cultural discontinuity, offers a stable means of communication. There are certain future scenarios, where natural language may become a most valuable means of communication. It has, however, clear limitations on any message content needing the precision of linguistic text. Only markers with durable pictograms and text, and off-site/on-site archives can provide this precision. Our site design will function best when complemented with more precise/specific modes of communication...but few other modes of communication have the durability and power of natural language.

\subsubsection{The Medium for Expression of Place-Archetypes}

The materials best used to manifest the content of place-archetypes must be the very stuff of place itself...that which differentiates place from all else. At its core, place is an earth-grounded, sky-covered, sheltering and surrounding physical spatiality that we inhabit and move through. From this, we can describe the basic elements from which places derive meaning.

In the realm of landscape and architectonic built-form the elements that constitute all built-form and thus their meanings are: landform; location; fixity; markedness; substantiality; orientations and direction; order, rhythm, and sequence; acknowledgement of celestial activity; center and boundaries; dimensions and shapes; parts and wholes; enclosure and openness; passage and penetration; views to and from; light and dark; time and movement; available energy; plant 
material and its cycles; building materials ordered and worked; surface manipulation; local altering of climate; relationship to the near surround; inhabitation by the one and the many; maintenance, care, and sacrifice; and use, retirement, and ruin.

There are messages that place-design can easily communicate. To speak of architecture and landscape architecture as a "natural language" helps us to understand its capacity to communicate, but does not help us to know how. If we use language as the "model" for a place's capacity to communicate, we misunderstand it. It speaks in a different way. Place-design can speak about all the following, and importantly for this project, about their opposites as well: the flight from Chaos to Cosmos, and an ordering of intransigent nature; transformation and ordering of materiality; locating and sheltering; a locus for inhabitation and dwelling; safety and security; stability; an investment of energy; aspiration; nurturance; a focus of care and maintenance; a declaring of value and values; and a way we represent ourselves to ourselves, and others.

The "language" is in and of form, and is multivalent, imprecise, and powerful. Yet, it is clearly possible to develop design guidelines for places to act as communications of ideas in a natural language of form. As an example, Brill [Ref. 3-4] developed design guidelines for sacred space that embody and tell myths of the creation of the world, following the research of Rapoport [Ref. 3-5] and Eliade [Ref. 3-6].

Some of the archetypal feelings and meanings we will explore in design of the markers for the WIPP site are those of: danger to the body; darkness; fear of the beast; pattern breaking chaos and loss-of-control; dark forces emanating from within; the void or abyss; rejection of inhabitation; parched, poisoned and plagued land...and others. In the Design Guidelines in Section 4, we describe the meanings we wish our site design and built-form to communicate. The possible origins of archetypes are discussed in detail in one of the appendices, Section 5.4.

\subsection{The appropriate use of graphics in marking}

By "graphics" we refer to design elements such as pictures, signs, drawings, pictographs, cartoons, icons, and symbols. If language fails, these may provide a powerful means for communicating our message. Even if language is understood, moreover, there are forms of information that can be more succinctly and successfully transmitted by means of graphics.

Graphics pose problems, however, that must be carefully considered in their design.

\subsubsection{Ambiguity}

Graphics are likely to be ambiguous. Even for people who share a culture, they can, in the absence of accompanying clarifying language, be subject to varying interpretations. Indeed, the Thematic Apperception Test, used in clinical psychology, exploits precisely this kind of ambiguity in a series of drawings of people in various situations, asking the person being tested 
to tell a story about what each picture seems to represent. The "biohazardous waste symbol" proposed by the Human Interference Task Force [Ref. 1-2] is an example of unintended ambiguity. Some people to whom we showed it said it seemed to suggest that one should dig in the direction of the downward pointing arrow. The symbol suggested digging claws to them.

\subsubsection{Removal}

Graphics are liable to be perceived as "art" and to be removed. Such perception becomes increasingly likely in the course of time as they become seen as relics of the art of a past civilization to be displayed in museums or sold to art collectors. Witness what happened to the stelae of the Maya.

\subsubsection{Cultural Restrictions}

Graphics are likely to be culturally restricted in meaning. There are no conventional signs, such as the skull and crossbones, ${ }^{1}$ for example, that convey the same meaning across cultures. A bar across a picture of someone digging may suggest prohibition of digging to people now, but one cannot be sure that it will not be seen as suggesting something positive about digging 3,000 years from now. Representations of human faces and human and animal figures tend to be recognized for what they are, however, across cultural boundaries and millennia. For example, we have no trouble recognizing such figures in the paleolithic cave paintings of Europe and in prehistoric rock carvings and rock shelter paintings in Africa, Australia, and the Americas. We can even recognize many of the activities in which the human figures in these paintings seem to be engaged. But why these representations were put there and what the beholders should infer from them are obscure and the subject of conflicting interpretations. Cross-cultural ambiguity of this kind is especially likely with the use of cartoons.

\subsubsection{Universal Expressions}

Representations of human facial expressions of emotion and feeling, such as pain, anger, disgust, and fear communicate in the same way universally, regardless of cultural differences. They recommend themselves, therefore, for appropriate use in the design of the marker system.

1 In Mexico, the bones are the repository of the life force, and thus the skull and crossbones would have a very different meaning. 


\subsection{The languages to be inscribed on markers}

\subsubsection{The Importance of Linguistic Markings}

While it is possible that the content of pictures, icons, symbols, and so on that are devised today might be recognizable to the average person 10,000 years from now, this is surely inconceivable for written language. Languages are in a continuous state of change; linguists have no ability to predict the course of this change and it is unlikely that they ever will. Certain changes in pronunciation and grammar are more likely to occur than others, but languages are such complex systems that any tendency to "simplify" one part of the system is likely to trigger complicating effects in another. As a result, there is no general directionality to language change. Also, many changes are effects of historical factors that no one can foresee. The primary reason that English differs so profoundly from its closest Germanic relative Frisian (spoken in the northern part of the Netherlands) is that speakers of the former, but not the latter, were conquered by French-speaking Normans.

As a consequence, only "experts" will be able to read written messages on the markers after a number of centuries. Even so, linguistic markings are more important than iconographic ones because the former are inherently less ambiguous. Again, barring some drastic cultural discontinuity, there will always be scholars capable of reading the major languages of the twentieth century. The existence of literally millions of texts (and accompanying grammar books, dictionaries, and so on) will ensure that. However, it is not so obvious that the symbols that seem obviously iconic to us today will be interpretable in centuries to come. For example, considerably more effort must be expended in finding out the meanings of the alchemical, zodiacal, and occult symbols that were in common use 500 years ago than of the words that they represent. We suspect that 500 years from now, it will be correspondingly easier to uncover the meanings of the English words "radioactivity"' and "hazardous waste" than of the symbols now used to denote them.

In conclusion, there must be written warnings as well as pictorial-symbolic ones.

\subsubsection{Which Languages Should be Chosen?}

Any decision about the languages of the markers must be based on a combination of factors, the most important being the languages spoken at or near the WIPP site itself and the desirability of having all waste-disposal sites around the world marked in the same languages.

\subsubsection{Linguistic Demography of the WIPP Site}

The language in daily use by the majority of the residents of Eddy County (in which both WIPP and the city of Carlsbad are located) is English. The county has a sizeable Hispanic population (although not as large as in other parts of New Mexico) with Spanish spoken by a minority of 
residents, most of whom are bilingual in English. The Mexican border, however, is only 150 miles away, and parts of west Texas and New Mexico in which Spanish predominates are even closer. All projections agree that the percentage of Spanish speakers in this area will increase steadily in the foreseeable future.

Eddy County is less than $1 \%$ Indian and does not contain a community of speakers of an Indian language. There is a Mescalero Apache reservation about 120 miles to the northwest, with about 1,800 speakers out of a population of 2,000. There is no actively used written language, however, and even the spoken language is severely threatened, as children are not learning it or are learning it imperfectly. The huge Navajo reservation occupies the opposite corner of the state from the WIPP site and extends into northeast Arizona. The Navajo language has 130,000 speakers out of a population of 170,000 , many of whom live in Albuquerque and other towns outside the reservation. The written language is in the healthiest condition of any indigenous to North America; newspapers and books are published in it. Given current trends, Navajo should last well into the next century; as only about a third of the children are becoming fluent speakers, however, it too must be considered threatened.

\subsubsection{The Choice of Languages}

Which languages should the messages be in? English and Spanish are obvious choices, by virtue of their being spoken in the area of WIPP and also being two of the most widely spoken languages in the world. Our feeling is that if the scholars of future millennia cannot read current English or Spanish, they won't be able to read any language of today. However, because there are good reasons to mark every radioactive waste site in the world identically, more languages should be represented. Those of the United Nations are obvious choices: Arabic, Chinese, French, and Russian, in addition to English and Spanish.

Markers in countries where none of the above is the local language (say, Japan) will also have to be marked in that language. This means that (assuming that at least some markers will have all languages represented) there will have to be space on the markers for a seventh language. We suggest that the seventh language on the WIPP site markers be Navajo. While the immediate area contains few if any Navajo speakers, marking in Navajo grants recognition to the fact that Native American peoples predominated in the area for many thousands of years. Also, Mescalero Apache, which is spoken relatively close to WIPP, is very closely related to Navajo.

It will be important to consult with the Navajos themselves to ensure that they feel that including a message in their language is appropriate. After all, they may see it as a patronizing attempt to appease them as one more desecration of what was once Indian land is carried out. That Native peoples might not have an automatic revulsion at the idea of marking the WIPP site in an indigenous language, however, is suggested by the fact that the President of the Mescalero Apache Tribal Council, Wendell Chino, has recently received a Department of Energy grant to investigate the possibility of storing radioactive waste on their reservation. 
There exists today a number of artificially constructed "international" languages, the most notable of which is Esperanto. Millions of people in dozens of countries have had some connection with this language, but the number of effective speakers is under 50,000 . Study and use of Esperanto has had its ups and downs. It peaked between the two world wars, and was especially popular in the smaller European countries. Its effective death knell was sounded when the U.S. and the Soviet Union joined forces to prevent it from becoming a working language of the United Nations. We see no prospect of a widespread adoption of Esperanto, and do not recommend it as a language of the markers.

\subsection{Public information at the WIPP site}

A marking system whose message is intelligible to the current community has a higher probability of long-term understandability than a marking system whose message is unknown or unintelligible to its present-day audience. In this section, we present several options for enhancing the present-day level of knowledge in order to plant the seeds for future understanding.

There is a specific purpose for including such efforts to inform the public as part of the marking system. The Futures panel identified pressure to drill for oil and gas to be intense over the next two centuries. (Beyond that, the sources will have been exhausted and other energy supplies must be found.) The period of active institutional control for which credit can be taken is 100 years. Therefore, there is a 100-year window when there may be intense pressure to drill at the WIPP site. This 100-year window comes at the beginning, when the wastes are most dangerous (particularly if high-level waste is ever included at WIPP).

No funding for these public information efforts is assumed beyond the 100-year period of active institutional control. A high level of awareness at the beginning of the 100 -year window will help protect the site during this period. In many cases, what is proposed below would have already been considered as part of the Department of Energy plans for public information and involvement for WIPP.

\subsubsection{Public Involvement in Marking and Publicizing WIPP}

Before a final decision is made on how to mark the WIPP site, a diverse sampling of local perception of proposed markers should be gathered. The sample should include a cross section of whites, blacks, and Indians; "Anglos" and Hispanics; men and women; and people from a wide range of social classes and occupations. Publicity about the site must be aimed effectively at the public generally, in all its diversity.

\subsubsection{Off-site Archiving}

Any mining or other venture which might tap the buried radioactive waste is likely to be initiated from a city at some distance from the WIPP site. All pertinent facts regarding WIPP should thus 
be filed with any governmental agency, mining company, and so on that we can imagine having an interest in exploiting the site. Given the prospect of increasing multi-national ventures, these bodies are as likely to lie outside the United States as inside. There is no way, of course, of guaranteeing that the relevant information will be passed on to successor bodies over the centuries; the best we can do is hope that it will be.

\subsubsection{Empty Space for Reinscription}

Blank spaces should be left on all structures capable of taking inscriptions to allow for reinscribing the message in the contemporary local languages or copying from other message bearing stones at the site in case of defacement (see Section 4.4.9.3 and Figure 4.3-18).

\subsection{References}

[3-1] Raphael, M. 1945. Prehistoric Cave Paintings. Translated by N. Guterman. The Bollinger Series IV. New York, NY: Pantheon Books.

[3-2] García Guinea, M.A. 1975. Altamira y el Arte Prehistórico de las Cuevas de Santander. Santander: Patronato de Cuevas Prehistóricas de Santander.

[3-3] Bataille, G. 1955. Lascaux, or the Birth of Art: Prehistoric Painting. Translated by A. Wainhouse. The Great Centuries of Painting. Lausanne, Switzerland: Skira.

[3-4] [Brill, M.] 1985. "Using the Place-Creation Myth to Develop Design Guidelines for Sacred Space, an address given to the annual Council of Educators in Landscape Architecture (CELA) Conference. University of Illinois, Urbana, IL, September 20, 1985. (Copy on file in the Nuclear Waste Management Library, Sandia National Laboratories, Albuquerque, NM.)

[3-5] Rapoport, A. 1982. "Sacred Places, Sacred Occasions and Sacred Environments," Architectural Design. Vol. 52, no. 9/10, 75-82.

[3-6] Eliade, M. 1954. The Myth of the Eternal Return. Bollingen Series 46. New York, NY: Pantheon Books. 
Appendix F: Team A Report 
Appendix F: Team A Report

\section{Criteria for a Marking System with Examples}

\subsection{Site design guidelines for a design of the entire site, so it is a major component of a system of messages}

The Design Guidelines herein will be largely performance-based, that is, they describe how the design must perform, rather than what it must look like or be made of. These guidelines can, in turn, be used as criteria to evaluate designs. Because performance-based design guidelines do not describe the design, but rather what the design must do, several alternative designs can be developed in response to the guidelines. We have developed designs using the design guidelines, both as a test of the utility of the guidelines and as an expression of the team's preferred solutions. Because all the designs cover the entire interment, and then some, we refer to them as "site designs." These designs are presented in Section 4.2.

The various site design issues may be listed as follows:

- The site must be marked.

- All levels of message complexity should be located on-site. Thus, communication vehicles for information at Levels I, II, III, and IV should be on the WIPP site and available to humans. As well, this team has developed specific message content for each level, presented later in Section 4.6.

- The design of the whole site itself is to be a major source of meaning, acting as a framework for other levels of information, reinforcing and being reinforced by those other levels in a system of communication. The message that we believe can be communicated non-linguistically (through the design of the whole site), using physical form as a "natural language," encompasses Level I and portions (faces showing horror and sickness) of Level II. Put into words, it would communicate something like the following:

This place is a message... and part of $a$

system of messages...pay attention to it!

Sending this message was important to us.

We considered ourselves to be a powerful culture.

This place is not a place of honor...no

highly esteemed deed is commemorated here

...nothing valued is here.

1 In this discussion and then later in the descriptions of the designs that test these design guidelines we will use the expression "the Keep" to define an area whose size and shape is the "footprint" or the vertical projection on the site's surface of the final interment area. Our team's analysis suggests that the final footprint may be larger than currently shown because of both migration of radionuclides in the salt and future expansion. 
What is here was dangerous and repulsive to us.

This message is a warning about danger.

The danger is in a particular location...

it increases towards a center...the

center of danger is here... of $a$

particular size and shape, and below us.

The danger is still present, in your time, as

it was in ours.

The danger is to the body, and it can kill.

The form of the danger is an emanation

of energy.

The danger is unleashed only if you

substantially disturb this place physically.

This place is best shunned and left uninhabited.

- All physical site interventions and markings must be understood as communicating a message. It is not enough to know that this is a place of importance and danger...you must know that the place itself is a message, that it contains messages, and is part of a system of messages, and is a system with redundance.

- Redundancy of message communication is important to message survivability. Redundancy should be achieved through: (a) a high frequency of message locations, permitting some to be lost; (b) making direct and physical links among message levels, that is, "co-presentation" of messages; and (c) multiple and mutually reinforcing modes of communication.

It is expected that the number of presentations of messages will decrease as the message complexity (or Level) increases. Thus, there will be many more presentations of Level II linguistic messages than of Level IV.

While the system of marking should strongly embody the principles of redundancy, at the same time the methods of achieving redundancy should be carefully designed to maintain message clarity. Redundancy should not be achieved at the expense of clarity.

- The method of site-marking must be very powerful to distinguish this place from all other types of places, so that the future must pay attention to this site. The place's physical structure should strongly suggest enhanced attention to itself and to its subelements. To achieve this, the volume of human effort used to make and mark this place must be understood as massive, emphasizing its importance to us. The site's constructions must be 
seen as an effort at the scale of a grand and committed culture, far beyond what a group or sect or organization could do.

About scale: "Scale" refers to the perceived size relationship between a human and something else (like a house or a chair or a site). When the size of a thing gets far larger than a person, changes in scale are not easily perceived or are experienced as irrelevant. Thus, there is little difference to a person at ground level whether an earthwork is 1 mile or 2 miles long. These distances are experienced as much the same. What we propose as a marking for this site is already at a scale where it could be somewhat smaller or larger with no loss of meaning. And further, if the design were to be replicated elsewhere, it could be (somewhat) scaled up or down with no loss of meaning.

- Vertical masonry markers alone are simply not enough to accomplish our purposes. They are not large enough, nor frequent enough, nor sufficiently distinguishing from other sites already so marked; and their use elsewhere may well make their use here somewhat trivial and certainly ambiguous. If only markers are used here, they will be seen as much like markers on other sites, which are generally sites of far less import, and also tend to be marked because they are honorific or commemorative, the opposite of the message we seek to send.

- Use a system of markings that utilizes the whole site as an enormous mark, and that includes: smaller markers; high points to climb to from which to view the entire site; walls and places to be in that co-locate viewers with messages... an organized environment. Consider the possible retention of a currently existing structure for symbolic purposes only, as a decaying massiveness.

As for use of existing-site structures, if we assume no active institutional control, the only current above-ground site structure that might endure for a substantial portion of the 10,000 years would be the thick-walled concrete "hot" cell. The other buildings will decay, or more probably be stripped of their valuable building materials for re-use.

The "hot" cell may be put to symbolic use by incorporating it into the site's design, as a mute artifact suggesting something "strong" that needed to be contained, although from its large door size, a thing that had to be easily accessible and thus was (probably) not treasure. And, because the "hot" cell's openings are randomly placed, rather than symmetrical, it would tend not to be mistaken for an honorific or privileged structure. If the "hot"' cell is kept, it should not be located in the geometric center of any open space, which would symbolically elevate its importance.

- While this system of markings should represent an enormous effort and investment of resources on our part, the construction itself should be of materials of little value, and the workmanship should not bestow any value through elegance of craft or artistry. Doing 
substantial work on materials of little value suggests that the place is not commemorative of phenomena highly valued by the culture that made it, but as marking something important yet quite unvalued...not a treasure, but its opposite...a location of highly devalued material ("dangerous garbage" or an "un-treasure").

- The place should not suggest shelter, protection or nurture...it should suggest that it is not a place for dwelling, nor for farming or husbandry. This would be most strongly communicated if the place obviously tries to deny inhabitation and utilization. It might best be designed as a place difficult to be in, and to work in...both actually and symbolically. Given this, the center of the place should reject rather than embrace. Any attractive focus on/near the center would suggest welcome, and by extension, occupancy and utilization.

- We believe there is no physical barrier we can devise that (some) future technology cannot breach, and any attempt to bar entry physically to the Keep can and will be breached (by cutting through it, going under it, or coming down from above). Thus, any "barrier" placed around the Keep can only be purely symbolic, and should be used to enclose it only in a spatial sense rather than to attempt a fortification or a security barrier.

- As to the meaning of "center": physically to mark the WIPP site in any way makes it a different place from the surrounding desert, and creates a "figure" against a "ground." It makes a center in the desert.

- For human beginnings, making a center ("here we are") is the first act of marking order (Cosmos) out of undifferentiation (Chaos). All further meanings of "center" derive from this original positive valence. The meanings of "center" have always been as a highly valued place or a gathering place...the holy of holies; the statue centered within the temple, itself centered within the settlement; the dancing ground; the sacred place as the physical and spiritual center of a people, etc. In this project, we want to invert this symbolic meaning, to suggest that the center is not a place of privilege, or honor, or value, but its opposite. In symbolic terms, we suggest that the largest portion of the Keep, its center, be left open, and few (if any) structures placed there, so that symbolically it is: uninhabited, shunned, a void, a hole, a non-place.

- As for the geometric center, placement of anything at dead-center of the Keep would suggest that it is of the utmost importance, occupying the place of greatest privilege. We do not believe there is any one thing that can or should play that role on this site. (For example, someone might suggest that the highest Level IV of information might be placed at the center. But because a Level IV message may be gibberish to some intruders, while a Level II message would be well understood, no level of message is more important than any other, and no particular message or level is important enough to occupy the most privileged location.) 
- Design of the entire site and its subelements should avoid those forms that humans regularly tend to use to represent the "ideal," "perfection," or "aspiration." Aspiring forms are sky-reaching verticals, the obelisk, for example. Ideal and perfect ones are the perfect forms of symmetrical geometry (spheres, pyramids, hexagons) and of regular crystalline structures or polyhedrons. If such forms are used, we suggest their perfection be undermined through substantial and obviously meant "irregularity," as if its builders knew about the ideal and perfection, but asserted that this place is not about them. More appropriate types of forms to use are amorphic or jagged and horizontal, a deliberate shunning of the values of "perfection" or "aspiration."

- A major site-delivered message is that this place is ominous, not to be disturbed. This Level II message can be delivered both through site design and through "reading walls," discussed later. Message levels will probably be delivered in a sequence, but no level of message is more valuable than another. The design should incorporate this parity of levels. While Level IV information is certainly the most complete and detailed of all our communications at the site, there are certainly plausible future scenarios under which it will be of less value than a Level II message, or even of no value at all, even if seen. Thus, Level IV is more complex, but not a more valuable message to us (or future people), and its location should symbolically bestow no more value or privilege on it than on other message levels.

- The design should provide a general sense of the magnitude, shape, and location of the original danger. Because there is no apparent danger at the site's surface, the design makes it clear that the danger is below and threatens to escape. The site design should also articulate that the dangerous material is bounded, has a substantial footprint that is of a certain shape. Going out from this on-surface imprint might be concentric bands designed to signify diminishing danger. It is not necessary to mark the Land Withdrawal boundary; it is a legal boundary that will be meaningless in a few centuries.

- The enormity of this site's undertaking and its shape should be visible and comprehendible in its entirety, as a panorama. A panorama, the "seeing-all" from an altitude, is an ancient human metaphor for knowing, and seeking it is natural. Thus, provide elevated points for site viewing (mound, ziggurat, tower...all of which can be climbed for viewing).

- The site-marking system should also function as a locator for multiple concepts of location and should:

locate the site in relation to local centers of population of our time (which may contain archives as part of the information system); 
locate this site in relationship to other disposal sites in the world;

locate the viewer ("you are here") on all three spatial axes in relationship to the entire site and its subelements, and to the hazard;

locate the construction of this site in time; locate all on-site positions of Level III and IV messages.

- The place should be understood as both special and ominous from the air and from a distance. This implies a scale of construction whose heights are substantially greater than dunes, and whose overall pattern strongly differentiates it from desert.

- Maintain an approach and access to the place; permit and welcome access while suggesting the possibility of danger. Approached from ground level, information about the danger of the place should be available before you enter the Keep. From any point in the Withdrawn Area, a person must perceive that there is a direction of more or less danger, a gradient. Because it is probable that you cannot "see" the whole place from the ground, each part you encounter must point to a beneficial direction towards which to move.

As for details of the place and markers, we note the following:

- Inscribed messages need to be protected from future tourists taking pieces home as souvenirs. While messages need to be visually accessible, they should not be physically reachable. Thus, consider messages engraved high on hard-to-climb markers; message walls separated from viewing positions by a greater-than-jumping-distance chasm, etc.

- Because today's languages are not expected to be comprehensible to people other than future language scholars, part of the linguistic message should be an urgent request to update linguistic messages, to re-inscribe the messages as languages change. The physical design of message places should suggest and welcome such reinscription, perhaps by providing a sequence of "empty" markers near the original ones, or empty spaces' on markers.

- Wherever, possible, use design principles in which the intended performance of something is not diminished as. it degrades or fails. So the design of the place, and its construction, materials and configurations should gain, rather than lose, communicative capacity as parts erode over time, or as pieces are removed. Erosion or dismantling should expose new messages or reinforce them. (For example, in a wall built of stones, also inscribe messages on the surfaces not exposed, adjacent to the faces of other stones, so if the stone is removed, 
fresh messages appear. Because they are the same messages, curiosity should be reduced about what the next stone says.)

- The shape of built-structures (markers, walls, sculptural forms...) should enhance their durability. For example, we might use curved and bowed forms to:

"dish" wind-driven sand, which otherwise acts as an eroder;

have no sharp corners or arises, which are the first parts of faceted forms to spall and erode;

use materials whose geometry makes them poorly suited for reuse as a building material elsewhere ...(shapes on which too much work would need to be done to make them geometrically suitable for re-use construction);

protect other forms whose durability is more important.

- Inscriptions of the simplest linguistic and pictorial messages (Level II) should occur with more frequency than Levels III and IV inscriptions, and many of them should be fully accessible to message-viewers, implying their placement at external locations. These frequently occurring inscription locations should be reasonably protected from direct attack by. eroding forces. As an example, for an inscription on a wall, consider locating a second wall, higher and wider than the inscribed one, in a position that protects the inscribed wall and yet permits comfortable viewing by a few people. This second wall is "sacrificed"; it will erode to save the other.

- As for location(s) of Level IV information: While there certainly should/will be off-site archives for Level IV messages, and their locations described on-site, there must be Level IV message(s) available at the site to guarantee its availability. Continued retention and maintenance of archives elsewhere imply a highly improbable level of institutional control. Thus, at the site, there should be several locations for Level IV messages.

- Design of Level IV message places must recognize that a Level IV message, in any one language, takes up far more space than all others (about 10 times more than of Level III) and also involves non-text graphics such as diagrams and tables, further increasing space needs. We expect fewer Level IV messages at the site and a lower level of redundancy for this most complex level of message. Thus, Level IV message must have a high probability of enduring 
at the site. Only things unexposed to climatic cycles of change can endure. Thus, we recommend that each location of Level IV information be contained in an enclosed room whose exterior surfaces are protected from wind erosion and change cycles. So, provide for concrete or stone rooms underground, or embedded in earth/rubble above ground. (See the design drawing of Level IV room, Section 4.3, Fig. 4.3-17.) Consider as well some Level IV rooms at successive depths, revealed over time through site activities at the site.

In these rooms, we recommend the following:

- Messages be engraved in stone, primarily on vertical surfaces.

- Periodic table of the elements and astronomical drawings be inscribed on tilted stones at table height, the tilt clarifying which is top and which is the bottom of drawings.

- Messages be of a type size and at a height readable by a standing or seated individual (an area of inscription between 3- and 8-feet high would be optimum for a standing person to read).

- Relationship between type size and viewing distance affects both legibility and the amount of wall space needed for messages.

- The principle of redundancy suggests that several layers of message-on-stone be available in case a future people removes a set for study.

- The message-on-stone layers should be of identical stone materials and shaped to reduce their desirability as a building material (perhaps with odd shaped edges and bumpy backs).

- Several entries to each room be provided, each of them a removable stone or concrete plug that can slide into/out of an opening about $21 / 2$ feet square, large enough for human entry but too small to remove stone message panels intact. These entries should be marked so that excavators can find them easily.

- Room size should be dependent only on type size and viewing distance, message length, and number of languages. The room's purpose is to be seen as entirely pragmatic, a "message center" rather than symbolic or sacred.

- Overall comprehension can be reinforced by prominent sculptural models of the site showing on-surface and sub-surface elements of the site and the original location of the waste. The models should have scale, in relationship to themselves, to a person, or to the site.

Following this presentation of overall Design Guidelines is a set of designs that act as examples of these guidelines in physical form; as tests of the efficacy of the guidelines; and as a presentation of this team's preferences in design. There are several major families of design, 
demonstrating the range of responses possible and, also, that the guidelines are capable of multiple interpretations.

As well, there are design drawings of a Level 4 underground room, above-ground message walls, and ways to make durable symbolic structures.

These Design Guidelines are further enriched by a more detailed analysis (in Section 4.4) of the endurance and behavior of materials and structures, both above ground and below ground.

\subsection{Design options}

Presented on p. F-60 are several alternative designs for the entire site, followed by designs for some particular spaces on it. These designs are based on the Design Guidelines just presented and thus act as tests of the efficacy of the guidelines. Of the many designs developed and reviewed, these are also the design solutions most preferred by the team. The designs utilize archetypal images whose physical forms embody and communicate meaning. We have given them names, both for identification and as verbal images for each. They are:

Landscape of Thorns (Figs. 4.3-1, 4.3-2)

Spike Field (Figs. 4.3-3, 4.3-4)

Spikes Bursting Through Grid (Figs. 4.3.-5, 4.3-6)

Leaning Stone Spikes (Fig. 4.3-7)

Menacing Earthworks (Figs. 4.3-8, 4.3-9)

Black Hole (Figs. 4.3-10, 4.3-11)

Rubble Landscape (Figs. 4.3-12, 4.3-13)

Forbidding Blocks (Figs. 4.3-14, 4.3-15).

Some designs use images of dangerous emanations and wounding of the body. Some are images of shunned land...land that is poisoned, destroyed, parched, uninhabitable, unusable. Some combine these images. All designs entirely cover or define at least the interment area, called here the Keep.

Shapes that hurt the body and shapes that communicate danger: Danger seems to emanate from below, and out of the Keep in the form of stone spikes (in Spike Field and Spikes Bursting Through Grid--Figs. 4.3-3 to 4.3-6 and Leaning Stone Spikes--Fig. 4.3-7), concrete thorns (in Landscape of Thorns--Figs. 4.3-1, 4.3-2), and zig-zag earthworks emanating from the Keep (in Menacing Earthworks--Figs. 4.3-8, 4.3-9). The shapes suggest danger to the body...wounding forms, like thorns and spikes, even lightning. They seem active, in motion out and up, moving in various directions. They are irregular or non-repetitive in their shape, location and direction. They seem not controlled, somewhat chaotic. 
In the three designs that use "fields" of spikes or thorns, these spikes or thorns come out of, and define the Keep, so the whole area that is dangerous to drill down into is so marked.

“Menacing Earthworks" (Figs. 4.3-8, 4.3-9): Immense lightning-shaped earthworks radiating out of an open-centered Keep. It is very powerful when seen both from the air and from the vantage points on the tops of the four highest earthworks, the ones just off the corners of the square Keep. 'Walking through it, at ground level, the massive earthworks crowd in on you, dwarfing you, cutting off your sight to the horizon, a loss of connection to any sense of place.

The large expanse of center is left open, with only two elements in it: the WIPP's existing thick-walled concrete hot cell, left to ruin; a walk-on world map showing locations of all the repositories of radioactive waste on earth and a 50-foot wide map of New Mexico (Fig. 4.3-16), with the WIPP site in the geometric center of the Keep. The entire map is domed in order to shed sand blown by the wind. Underneath the slightly domed map a Level 4 room is buried (Fig. 4.3-17). Four other rooms are located under the four tallest earthworks. Reading walls (Fig. 4.3-18) are strewn between the earthworks, encountered before the Keep is entered.

Shunned land...poisoned, destroyed, unusable:

"Black Hole" (Figs. 4.3-10, 4.3-11): A masonry slab, either of black Basalt rock, or black-dyed concrete, is an image of an enormous black hole; an immense nothing; a void; land removed from use with nothing left behind; a useless place. It both looks uninhabitable and unfarmable, and it is, for it is exceedingly hot part of the year. Its blackness absorbs the desert's high sun-heat load and radiates it back. It is a massive effort to make a place that is fearful, ugly, and uncomfortable.

The heat of this black slab will generate substantial thermal movement. It should have thick expansion joints in a pattern that is irregular, like a crazy-quilt, like the cracks in parched land. And the surface of the slab should undulate, so as to shed sand in patterns in the direction of the wind.

"Rubble Landscape" (Figs. 4.3-12, 4.3-13): A square outer rim of the caliche layer of stone is dynamited and bulldozed into a crude square pile over the entire Keep. This makes a rubble-stone landscape at a level above the surrounding desert, an anomaly both topographic and in roughness of material. The outer rim from which rubble was pushed inwards fills with sand, becoming a soft moat, probably with an anomalous pattern of vegetation. This all makes for an enormous landscape of large-stone rubble, one that is very inhospitable, being hard to walk on and difficult to bring machinery onto. It is a place that feels destroyed, rather than one that has been made.

"Forbidding Blocks" (Figs. 4.3-14, 4.3-15): Stone from the outer rim of an enormous square is dynamited and then cast into large concrete/stone blocks, dyed black, and each about 25 feet on a side. They are deliberately irregular and distorted cubes. The cubic blocks are set in a 
grid, defining a square, with 5-foot wide "streets" running both ways. You can get "in" it, but the streets lead nowhere, and they are too narrow to live in, farm in, or even meet in. It is a massive effort to deny use. At certain seasons it is very, very hot inside because of the black masonry's absorption of the desert's high sun-heat load. It is an ordered place, but crude in form, forbidding, and uncomfortable.

Some blocks can be of granite, or faced with it, and carry inscriptions. Their closeness to other blocks reduces their exposure and increases their durability.

Note our use of irregular geometries and the denial of craftsmanship. None of our designs use any of the regular or "ideal" geometric forms, and only crude craftsmanship is sought, except for the precision of engraved messages. Why? The geometry of ideal forms, like squares and cubes, circles and spheres, triangles and pyramids is a fundamental human invention, a seeking of perfection in an imperfect world. Historically, people have used these ideal forms in places that embody their aspirations and ideals. In our designs, there is much irregularity both of forms and in their locations and directions, yet done by people with obvious knowledge of pure geometry. This shows an understanding of the ideal, but at the same time a deliberate shunning of it...suggesting we do not value this place, that it is not one that embodies our ideals.

The same is true of craft and workmanship. Historically, people use good workmanship to bestow value on things they value. In most of our schemes, the structures that cover or define the Keep's "cover" are made crudely, or of materials that prohibit workmanship (such as rubble, or earthworks, or a large slab). At the same time, we make an enormous investment of labor in these rude materials. It speaks of a massive investment, but one not tinged with pride or honored with value-through-workmanship.

About durability: All the designs, except one, have a high probability of lasting 10,000 years. This is because of their conformity with the guidelines for materials durability in Section 4.4.

The concrete structures of the Landscape of Thorns have projecting, cantilevered elements that will have tension on their upper surfaces, causing minute cracks. These cracks will accelerate local decay. Until new materials are available, or new methods for tensioning concrete members, we cannot "guarantee" the durability of this design. However, we present it here because of its strong emotive character. 
Appendix F: Team A Report

\subsection{A visual depiction of various design options}

Pages F-61 to F-78 show various designs described in Section 4.2. These designs are:

Figure $4.3-1 \quad$ Landscape of Thorns, view $1 \ldots \ldots \ldots \ldots$ F-61

Figure $4.3-2 \quad$ Landscape of Thorns, view $2 \ldots \ldots \ldots \ldots \ldots \ldots$ F-62

Figure $4.3-3 \quad$ Spike Field, view $1 \ldots \ldots \ldots \ldots \ldots \ldots \ldots \ldots$ F-63

Figure $4.3-4 \quad$ Spike Field, view $2 \ldots \ldots \ldots \ldots \ldots \ldots \ldots \ldots$ F-64

Figure $4.3-5 \quad$ Spikes Bursting Through Grid, view $1 \ldots \ldots \ldots \ldots$. . . . . .

Figure 4.3-6 Spikes Bursting Through Grid, view $2 \ldots \ldots \ldots$. . . F-66

Figure $4.3-7 \quad$ Leaning Stone Spikes . . . . . . . . . . . . . . . F-67

Figure $4.3-8 \quad$ Menacing Earthworks, view $1 \ldots \ldots \ldots \ldots \ldots$ F-68

Figure 4.3-9 Menacing Earthworks, view $2 \ldots \ldots \ldots \ldots$. . . . . . . . . .

Figure $4.3-10 \quad$ Black Hole, view $1 \ldots \ldots \ldots \ldots \ldots \ldots$. . . . . . . . .

Figure $4.3-11 \quad$ Black Hole, view $2 \ldots \ldots \ldots \ldots \ldots \ldots$. . . . . . . . .

Figure $4.3-12 \quad$ Rubble Landscape, view $1 \ldots \ldots \ldots \ldots \ldots \ldots$ F-72

Figure $4.3-13 \quad$ Rubble Landscape, view $2 \ldots \ldots \ldots \ldots \ldots \ldots$. . . . . . . F3

Figure $4.3-14 \quad$ Forbidding Blocks, view $1 \ldots \ldots \ldots \ldots \ldots$ F-74

Figure $4.3-15 \quad$ Forbidding Blocks, view $2 \ldots \ldots \ldots \ldots$ F-75

Figure 4.3-16 Walk-on Map of World's Radioactive Burial Sites . . . . . . F F-76

Figure 4.3-17 Buried Room with Level IV Messages $\ldots \ldots \ldots \ldots$. . . . F-77

Figure $4.3-18 \quad$ Reading Walls/Message Kiosk $\ldots \ldots \ldots \ldots \ldots \ldots$ F-78 


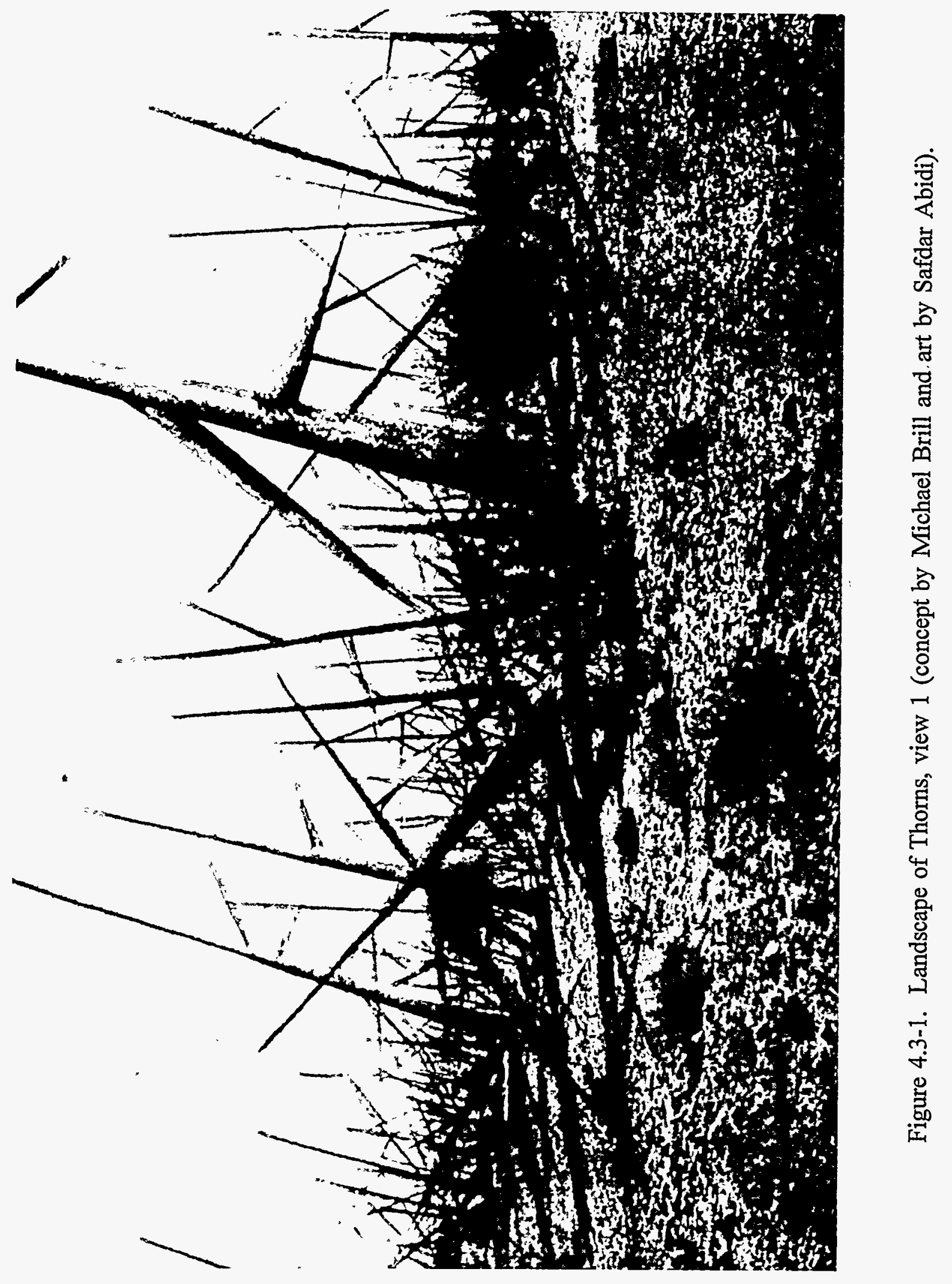




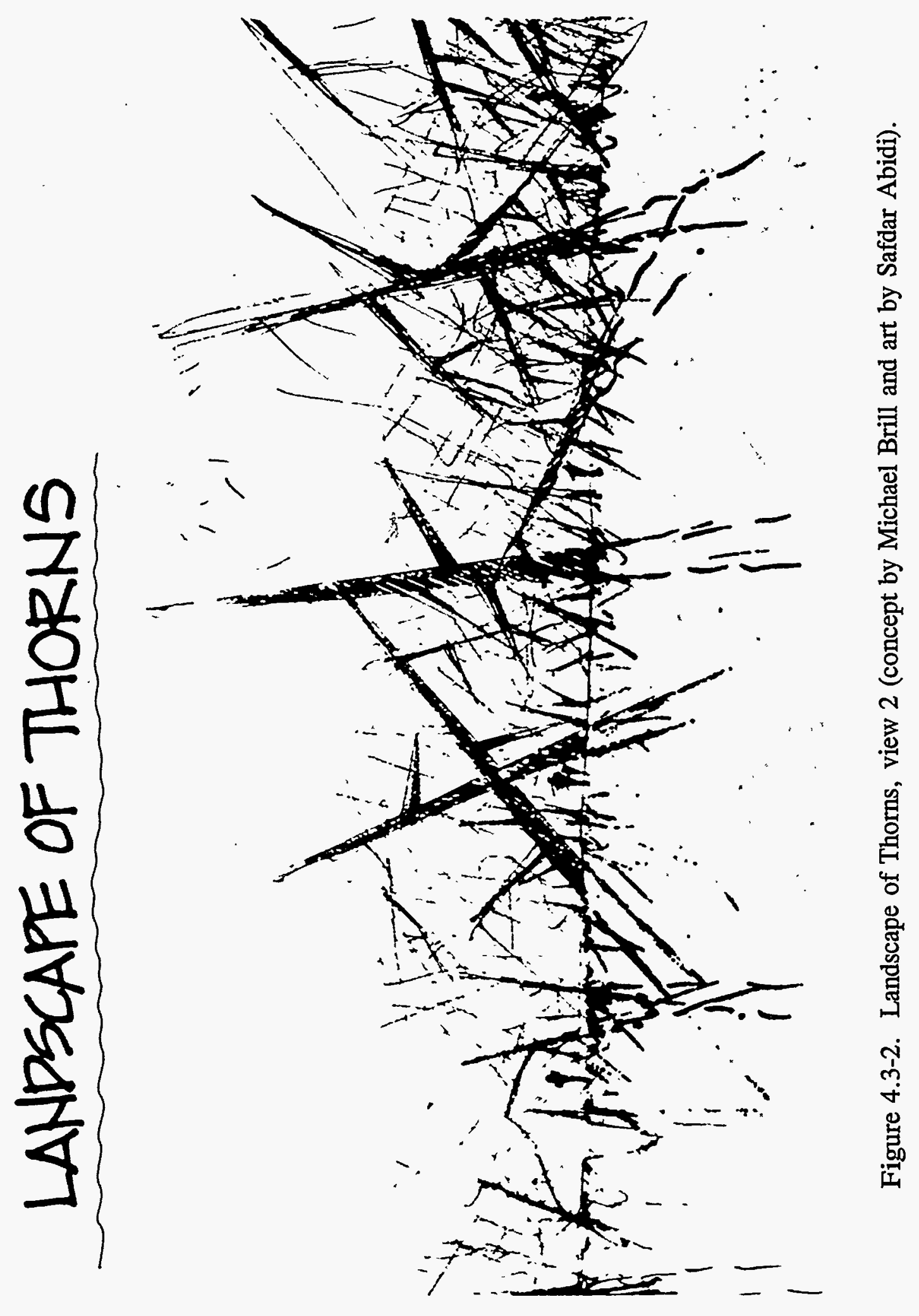




\section{SPIKE FIELD}

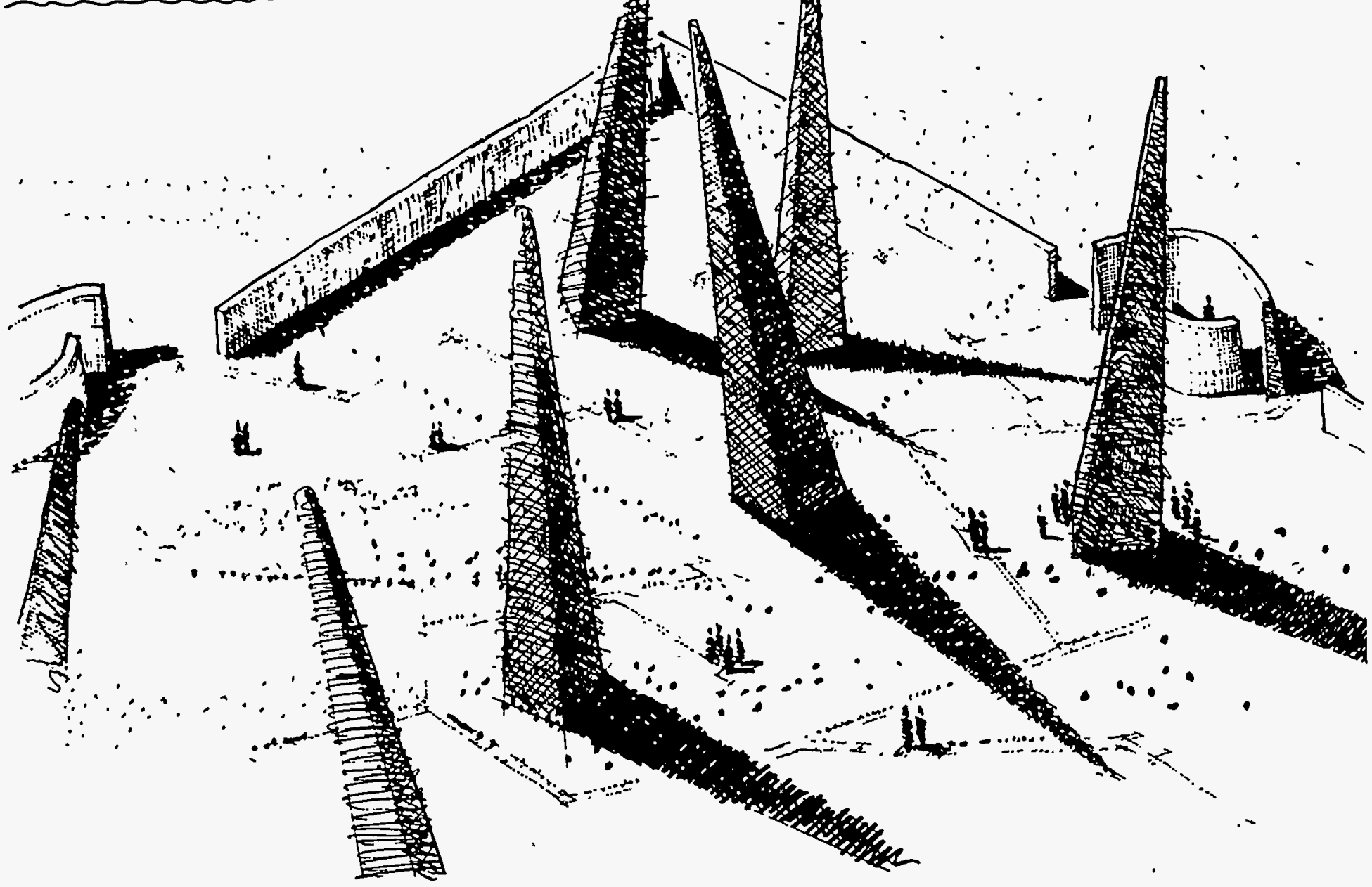

Figure 4.3-3. Spike Field, view 1 (concept and art by Michael Brill). 


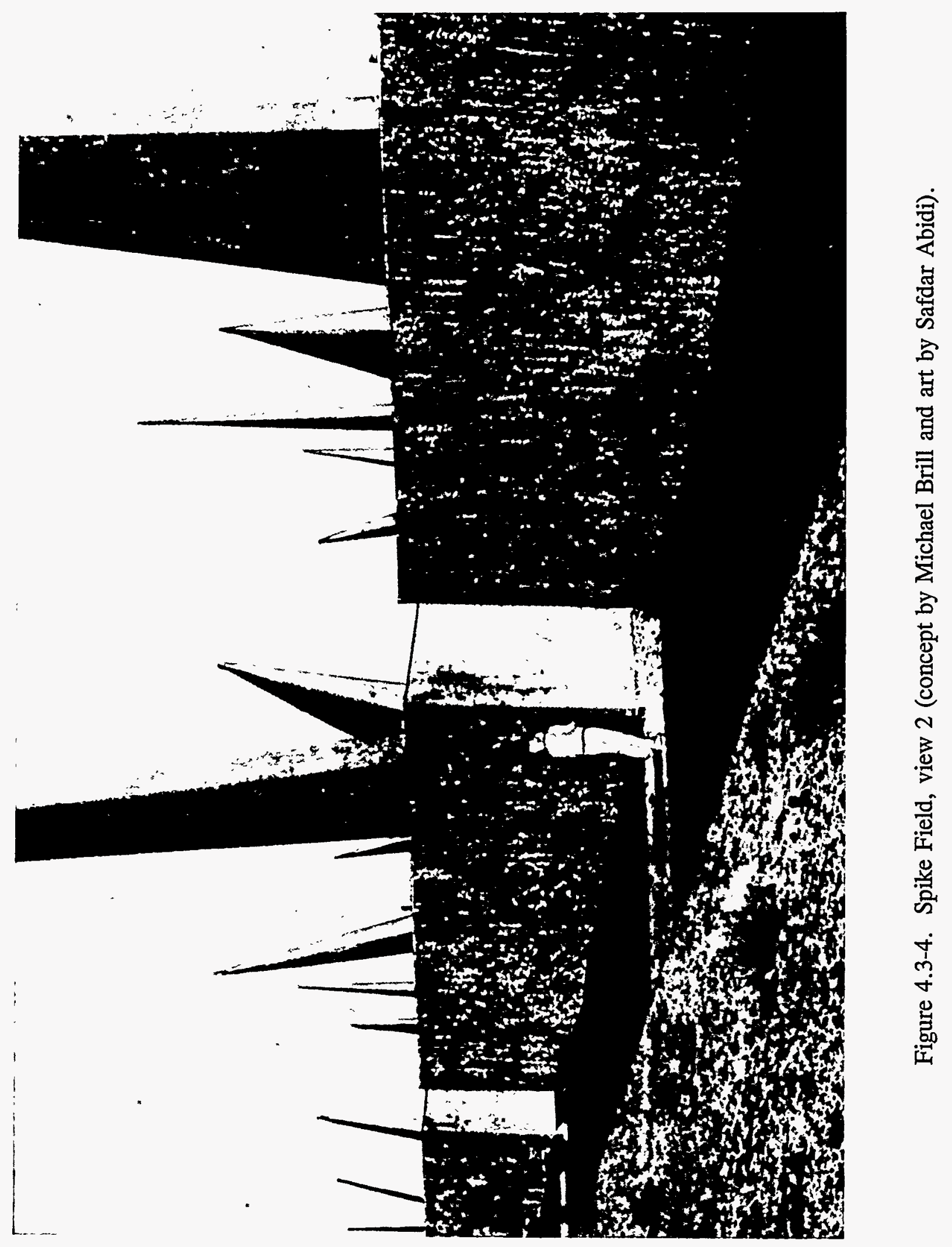




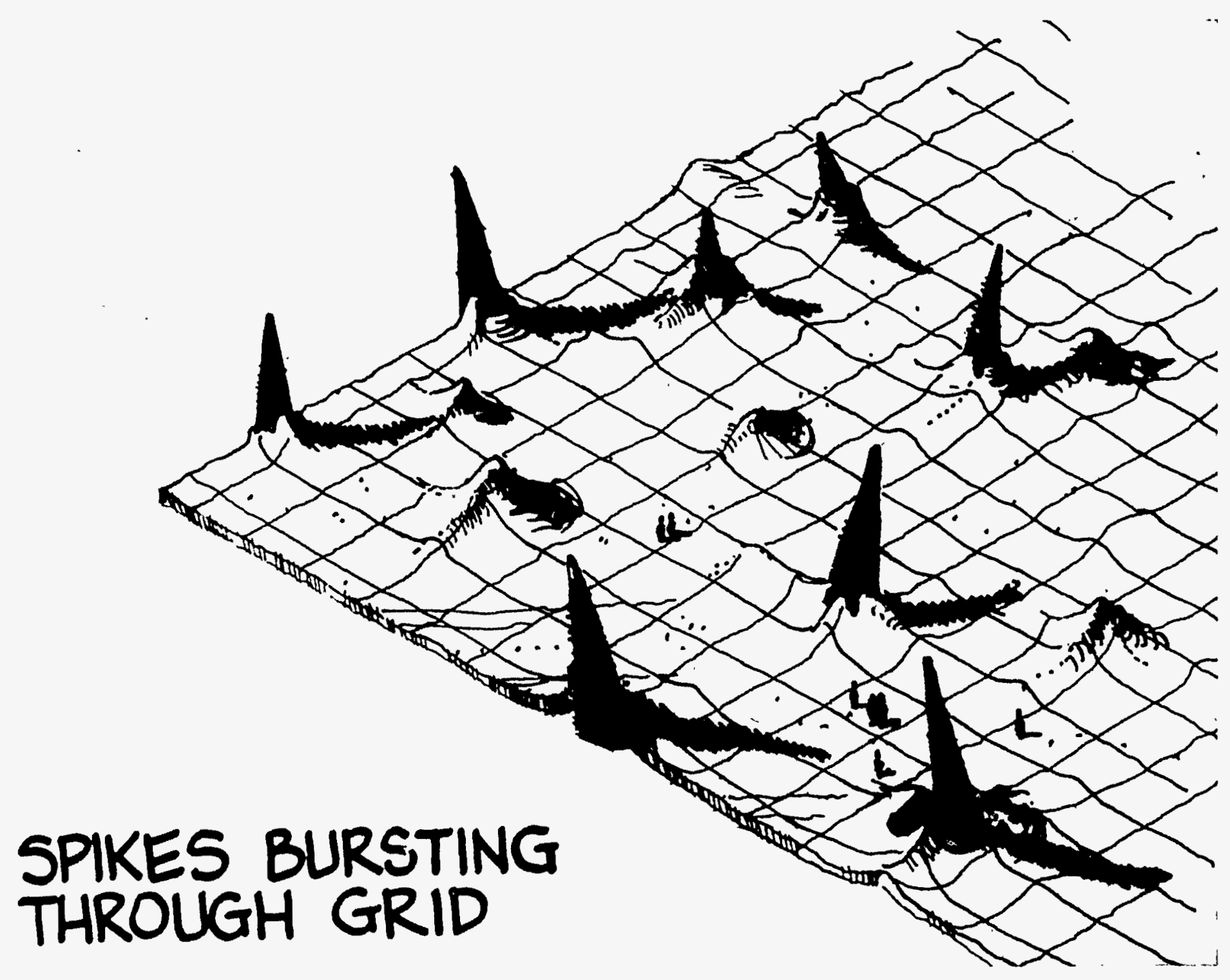

Figure 4.3-5. Spikes Bursting Through Grid, view 1 (concept and art by Michael Brill). 


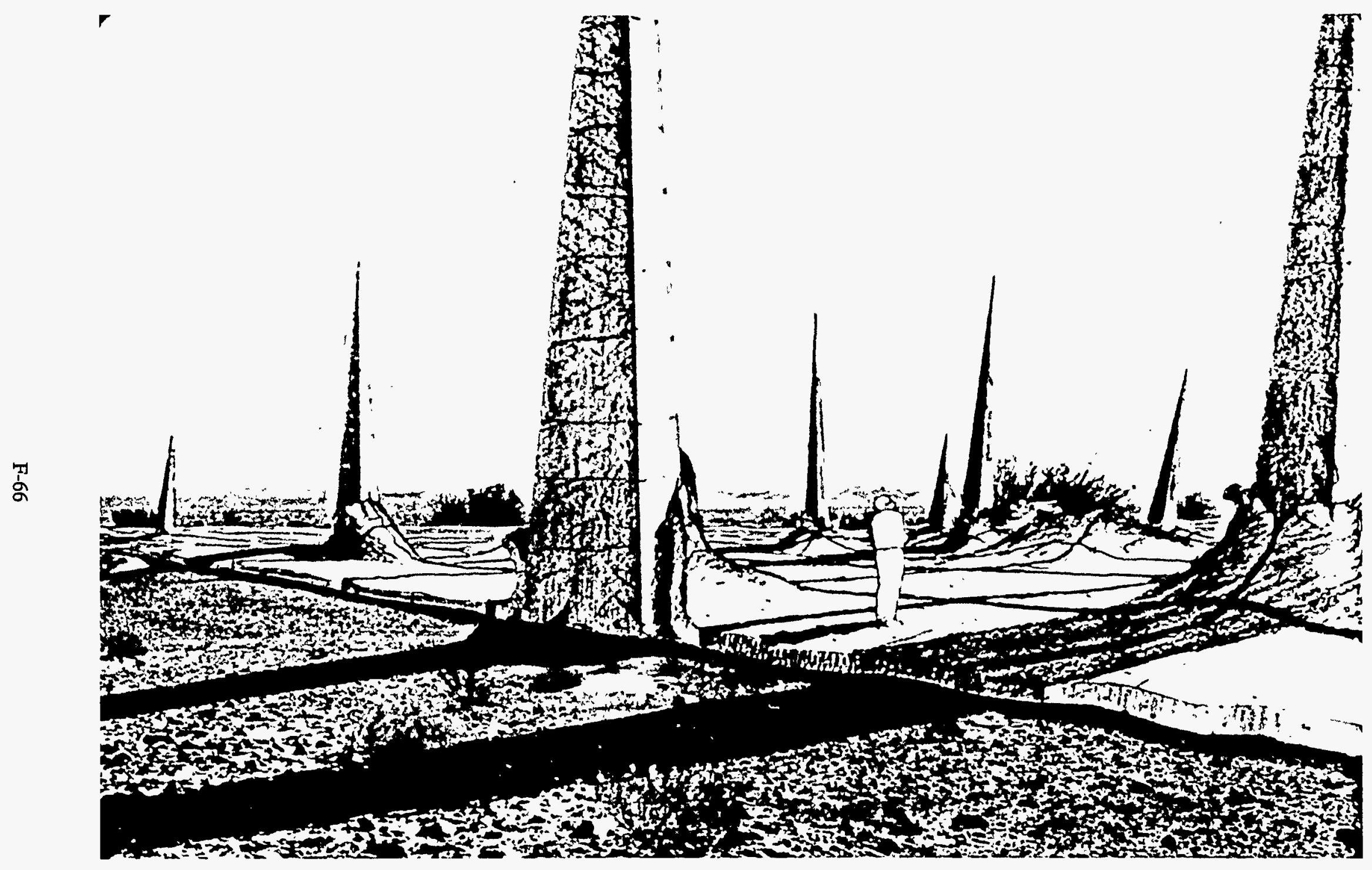

Figure 4.3-6. Spikes Bursting Through Grid, view 2 (concept by Michael Brill and art by Safdar Abidi). 


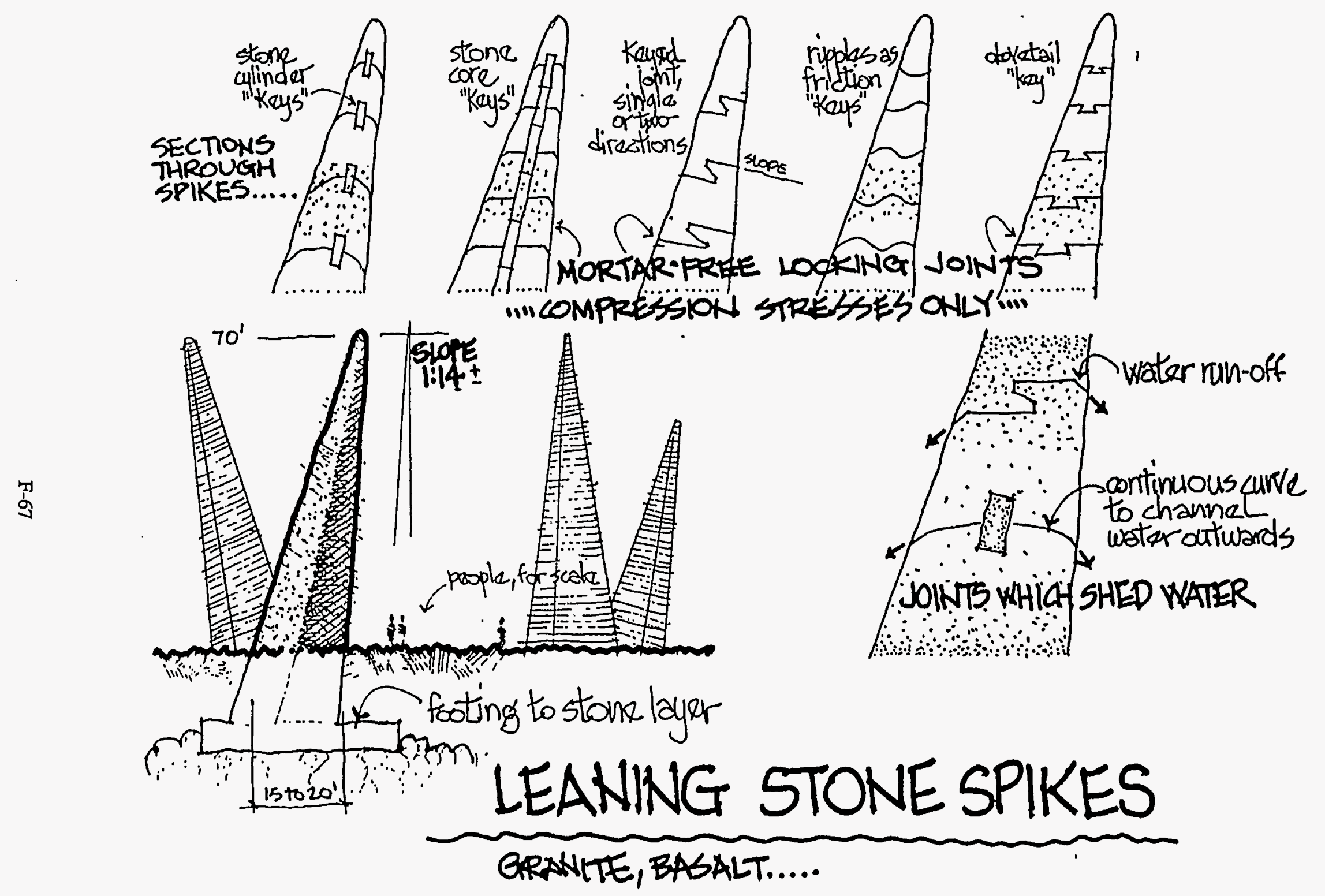

Figure 4.3-7. Leaning Stone Spikes (concept and art by Michael Brill). 


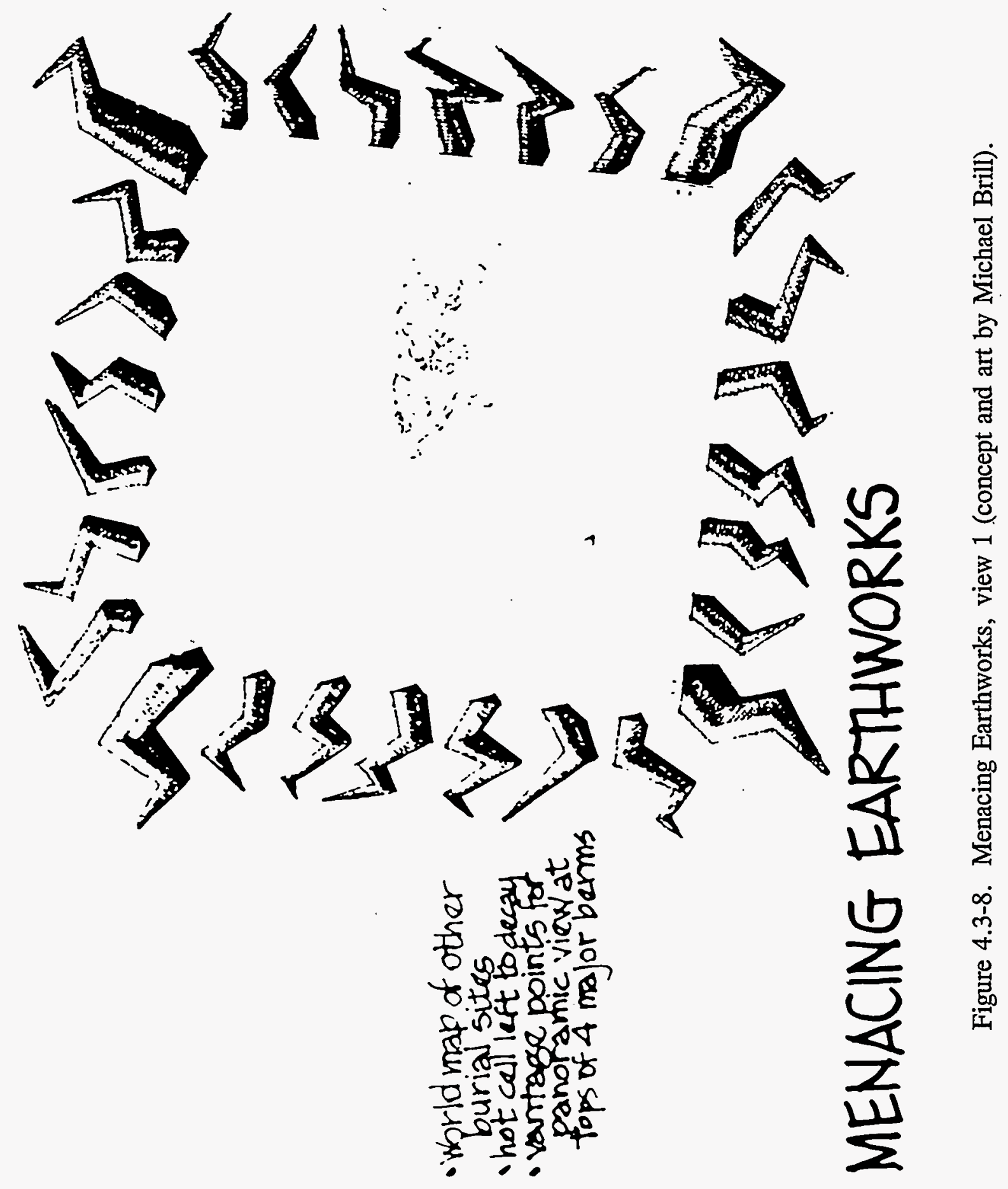




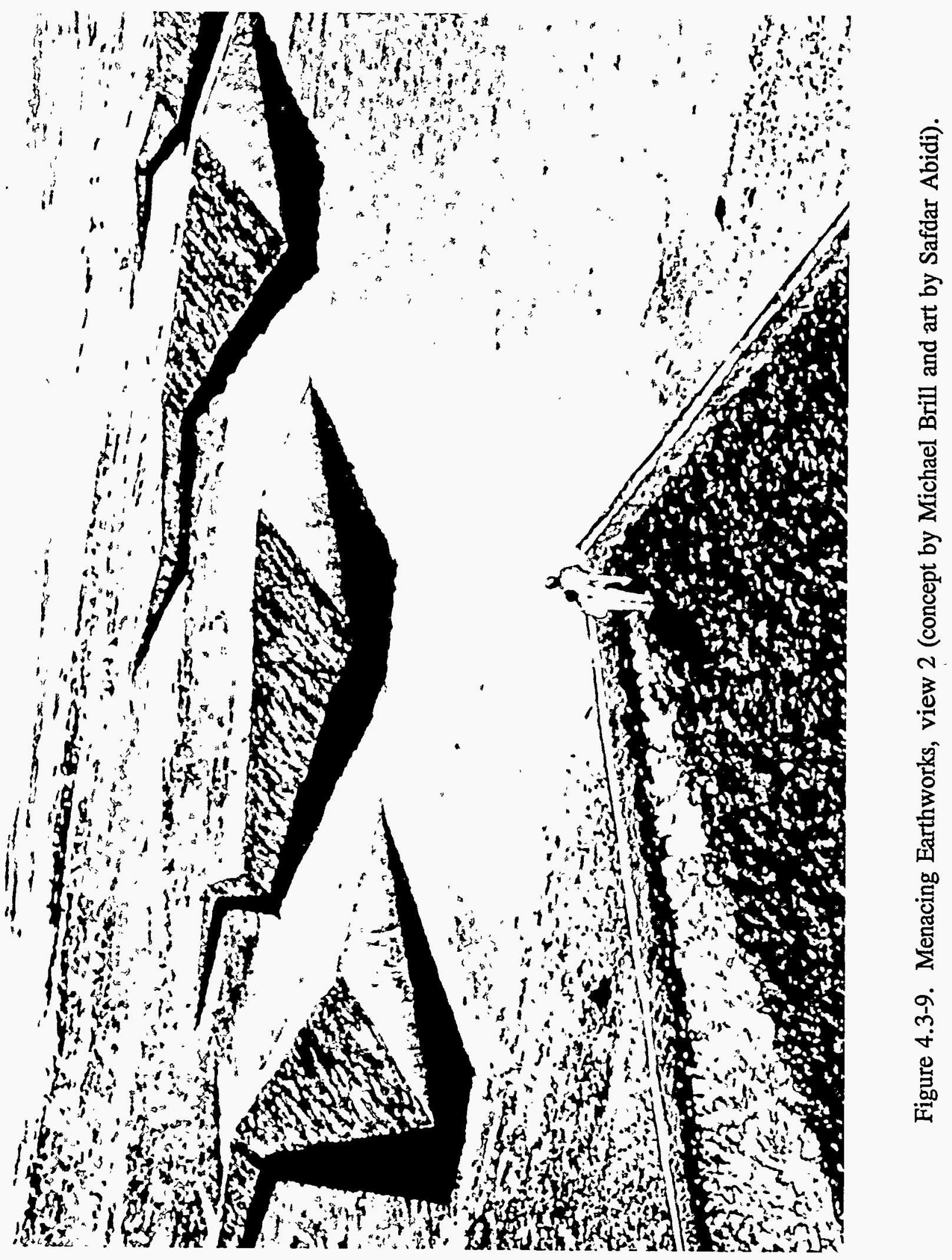




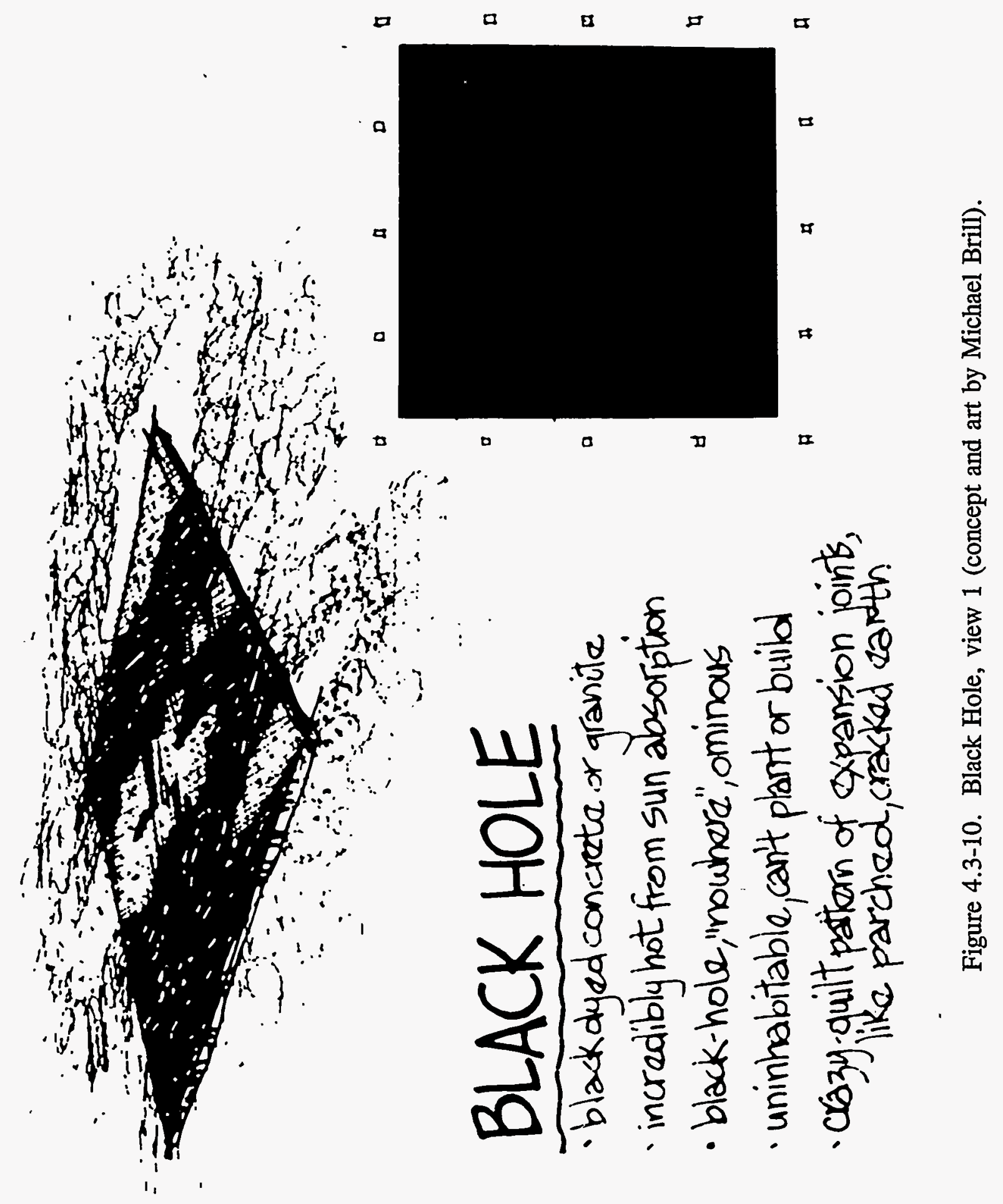




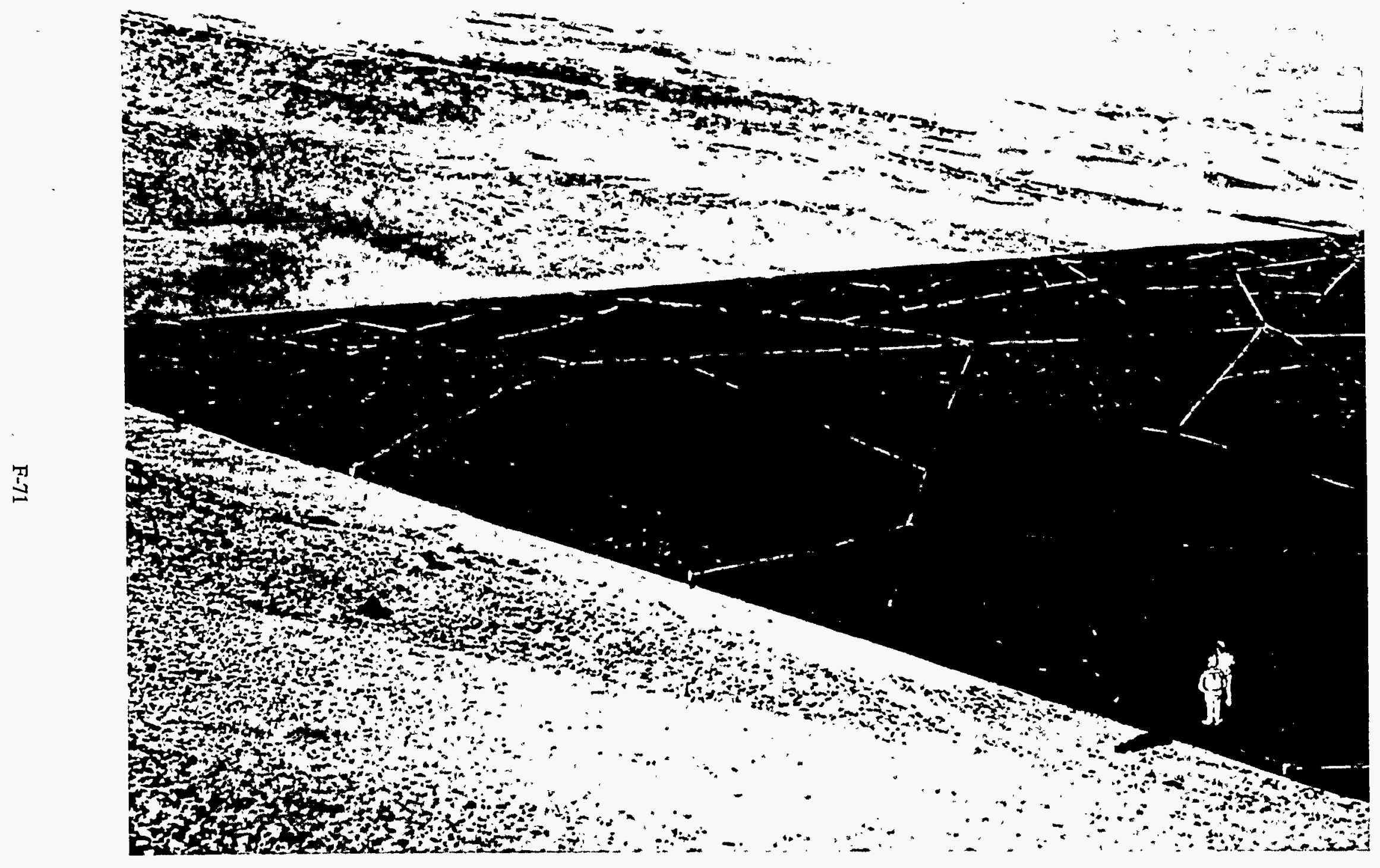

Figure 4.3-11. Black Hole, view 2 (concept by Michael Brill and art by Safdar Abidi). 


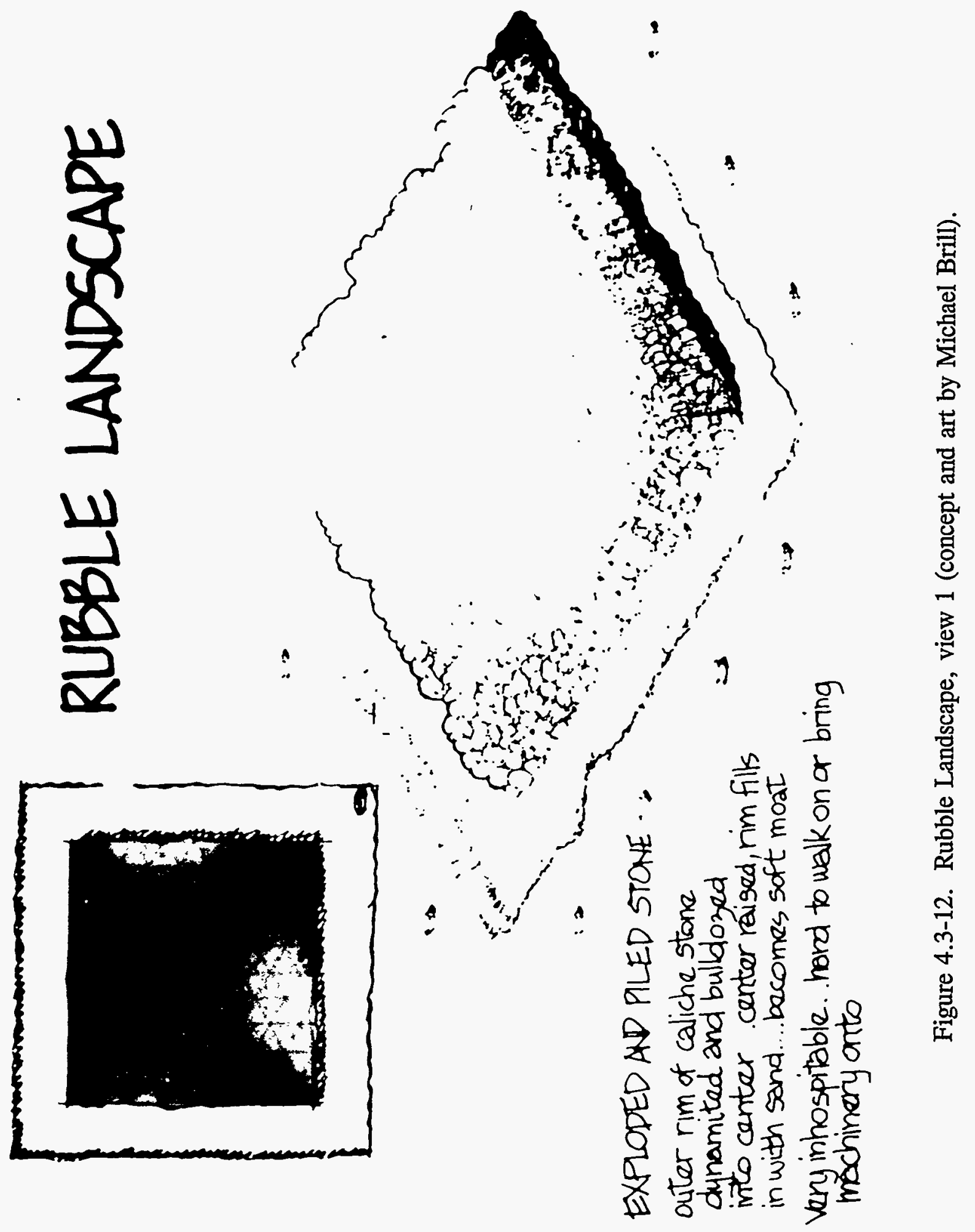




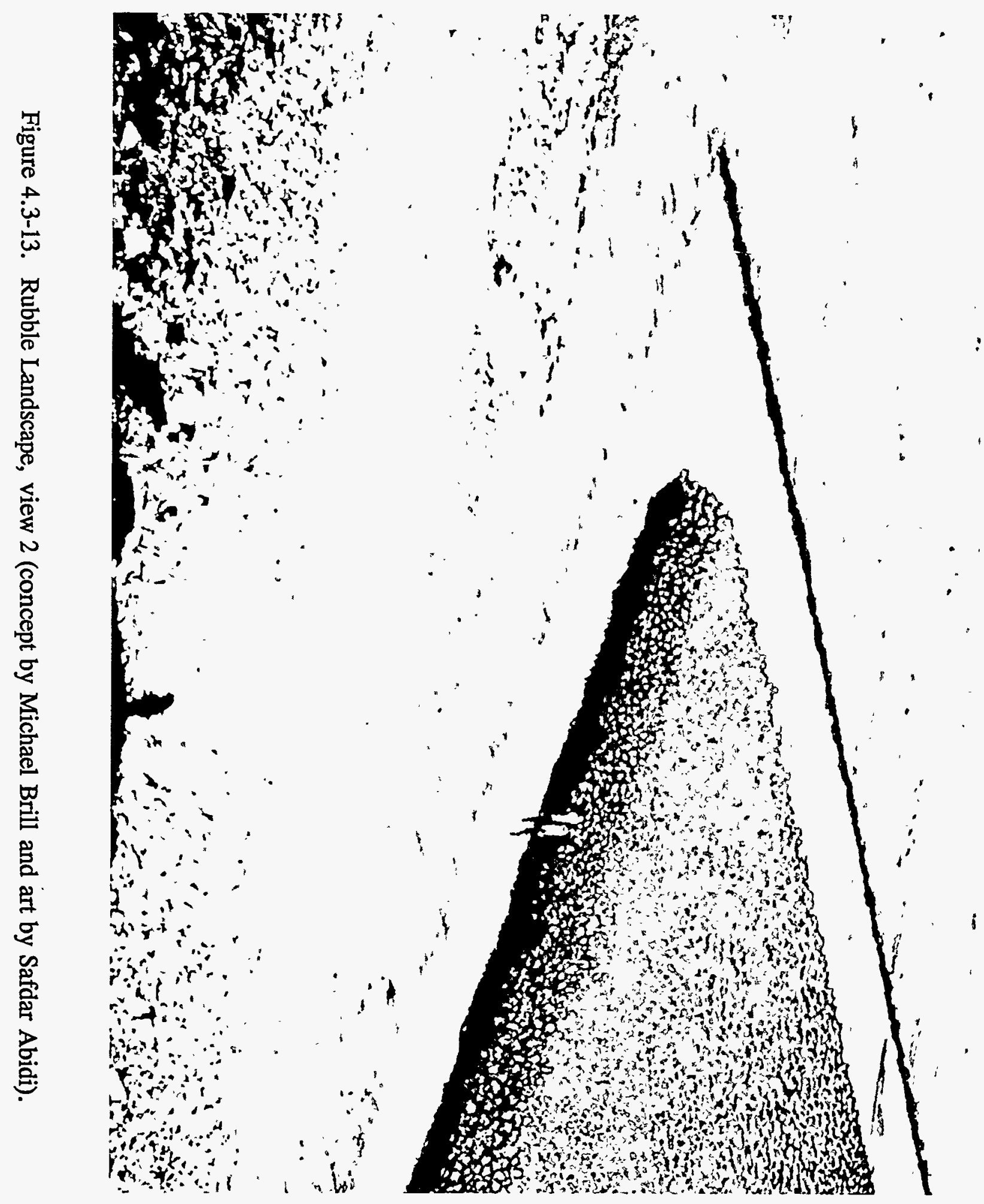




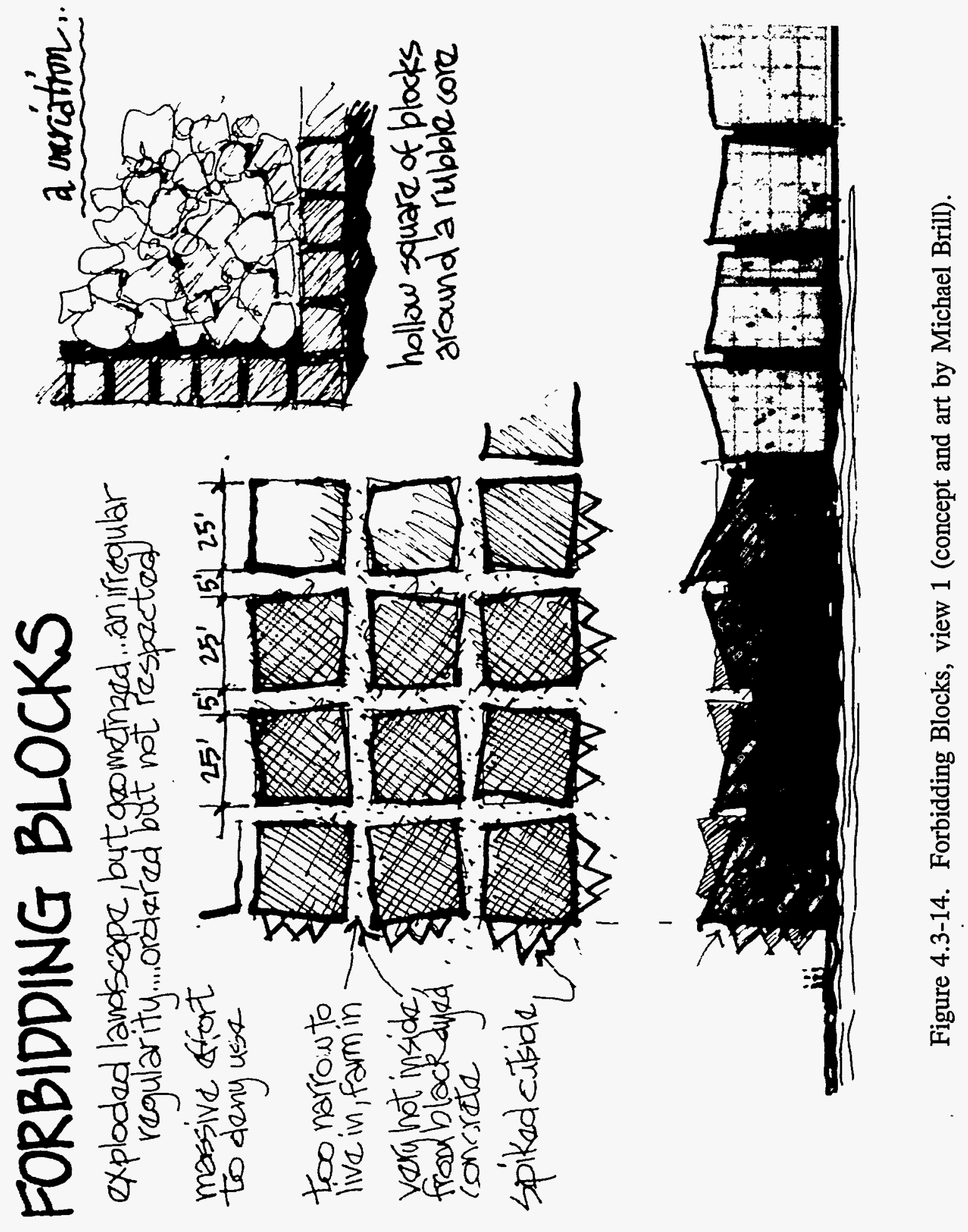




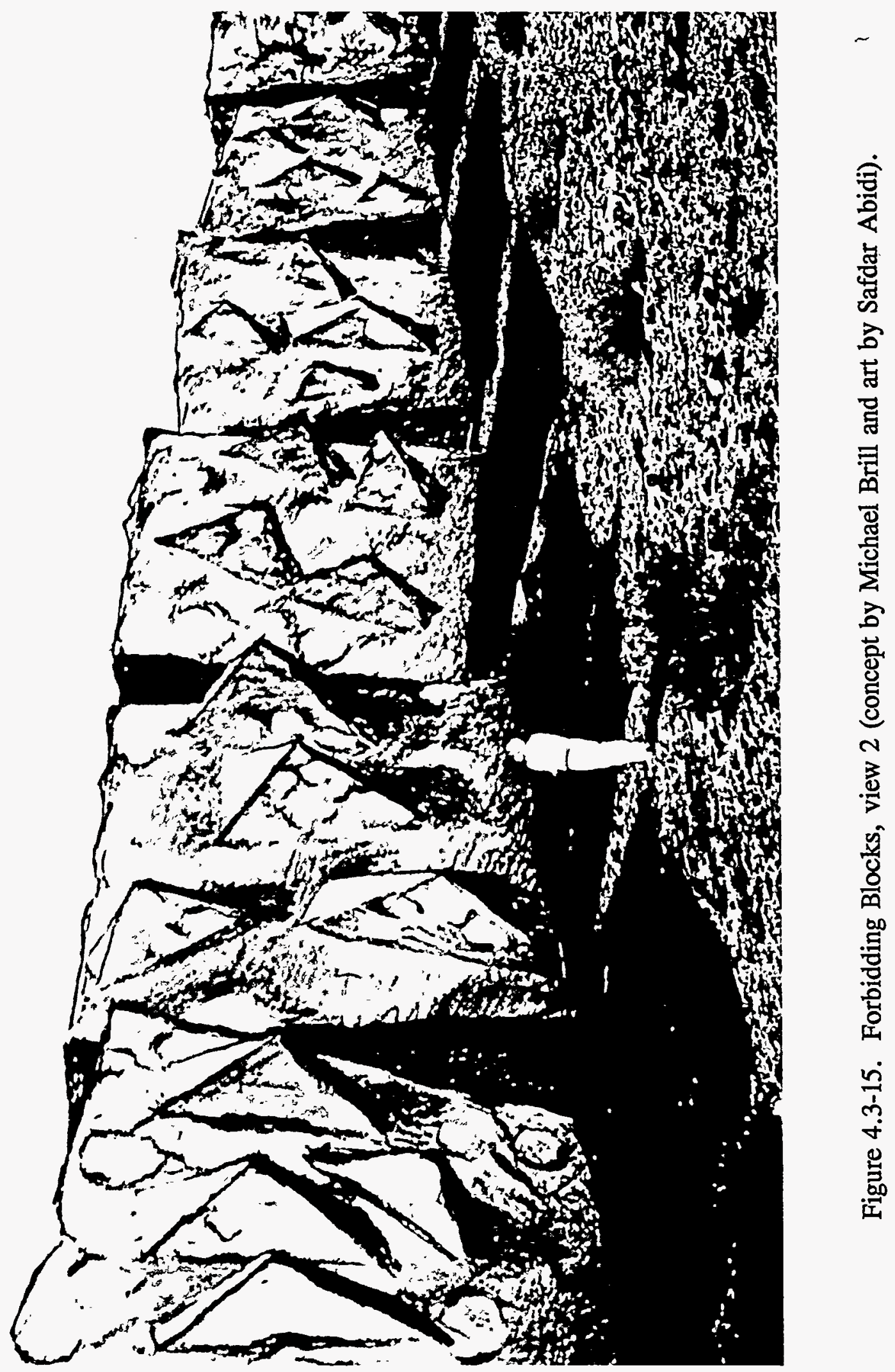




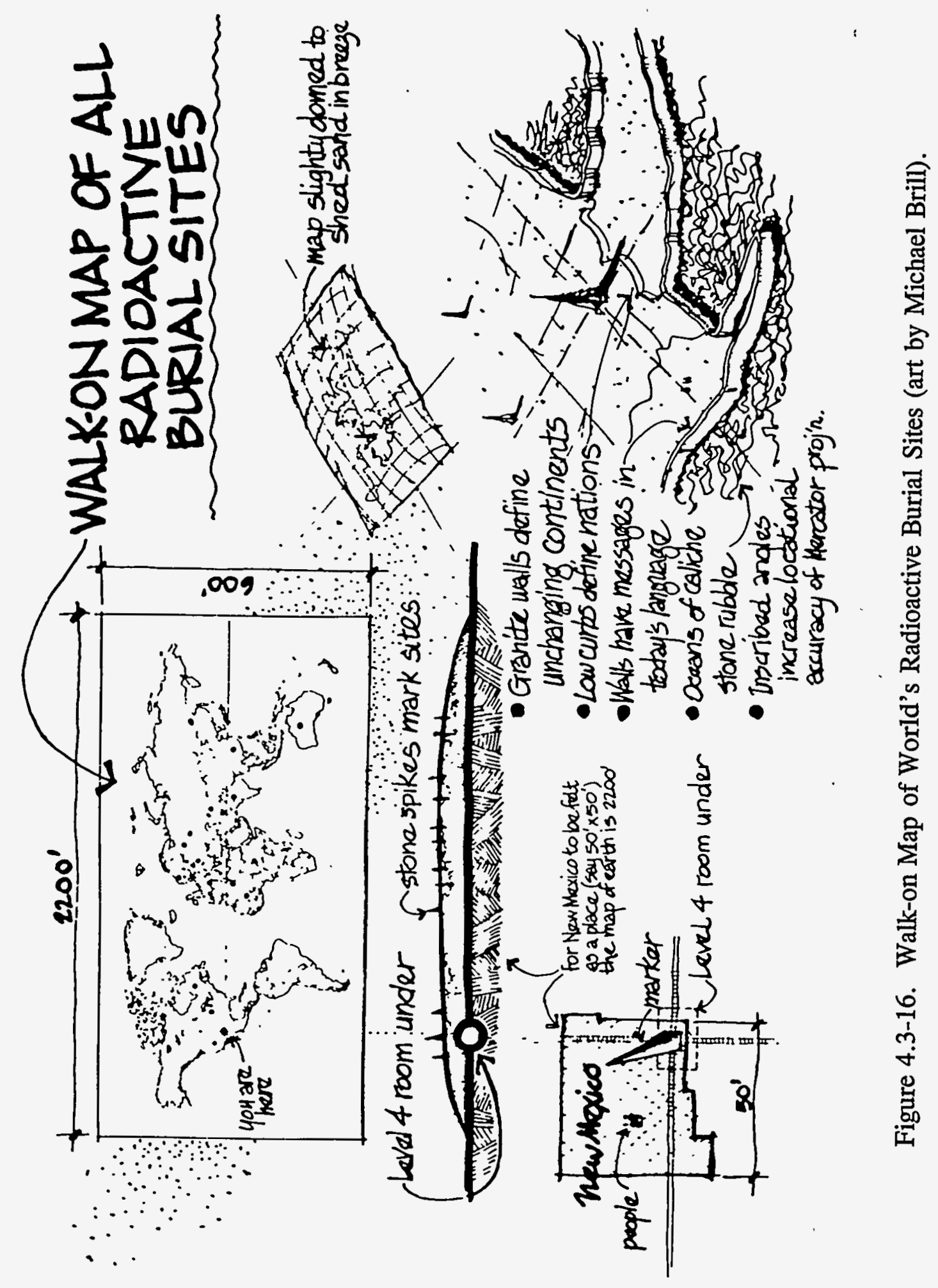




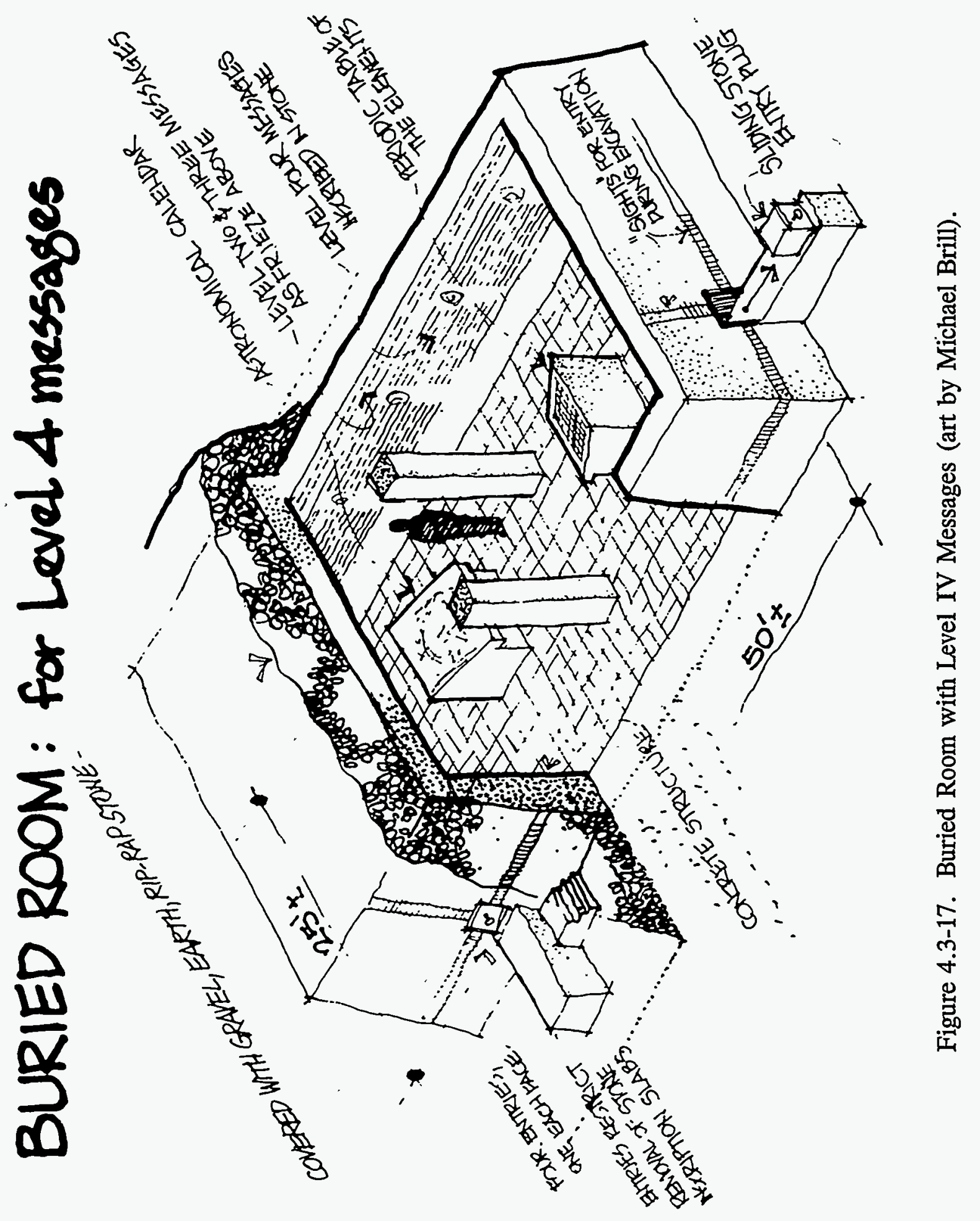




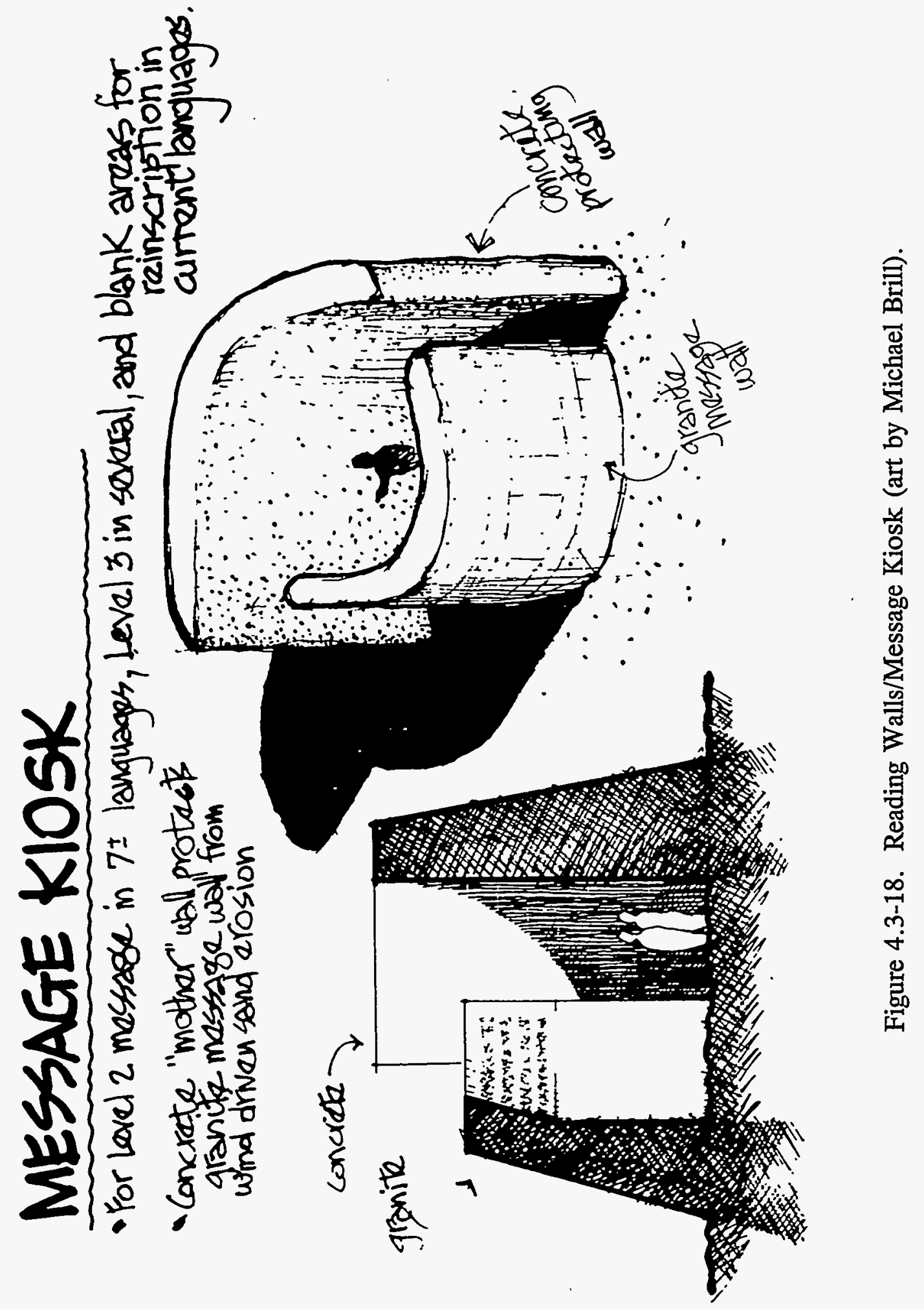




\subsection{Durability of common marker structures}

\subsubsection{Introduction}

This section discusses the durability of various proposed above-ground and below-ground marker structures.

The charge to the panel is to make recommendations to mark the site with "durable" markers. In what follows we take the position that "durable" in addition to meaning "resistant to decay by forces of nature" shall mean "resistant against removal by man."

A marker system designed to be "durable" against attempts of individuals to remove or deface markers (vandalism, recycling) can also be designed to offer very different degrees of resistance against removal by future societies.

Various scenarios can be envisaged under which future governments would want to remove the marking system, either in order to increase the economic value ${ }^{2}$ of the site or to deter advertent intrusion into the WIPP. ${ }^{3}$

On balance, the team recommends a marker system designed to be as difficult as possible to remove by future societies.

\subsubsection{Should all Markers be Durable?}

Clearly, some markers at the site must endure for 10,000 years. However, this does not imply that all markers must endure for such time. For example, if we accept the prediction of the Futures panel that drilling for oil and gas will cease within the next 200 years, then it would make sense to design a sub-set of markers with a design life of 300 years, containing specific warnings--in English and Spanish only--against drilling for hydrocarbons at the site.

Furthermore, such markers could be designed to shield for some time the more elaborate, complex and durable markers required to warn societies in the more distant future. Thus, the wooden structures bearing warnings not to drill at the site might contain monolithic cores of granite inscribed with the full set of Level II and III messages.

2 Value of land increases when knowledge that toxic material is buried there becomes lost. This results in a tendency by local government bodies to lose, destroy, or forget such information, see [Ref. 4-1].

3 Imagine, for example, a border conflict in which a temporarily victorious party controlling the WIPP site decides to contaminate the area before retreating by drilling into and through the waste panels. The degree of contamination, of course, would depend very much on the amount of high pressure brine, if any, released by the drilling. This scenario, although not considered by the Futures panel, is plausible (see, e.g., Iraqi behavior in Kuwait) and appears repeatedly in military history as the "scorched earth" tactic. A "removable" marker system could have been dismantled by the society owning the site, making it more difficult to locate the WIPP site. 
Appendix F: Team A Report

\subsubsection{Categories of Markers}

We shall use the term marker both for structures that are messages in themselves ("something man-made is here') and for structures that provide space for graphic and written messages. In addition, buried structures designed to introduce anomalies in the gravimetric, seismic, electrical conductivity, and magnetic profiles of the site are considered to be markers, because they will help to locate the site even if all surface structures were to be obliterated by unforeseen events.

\subsubsection{Distinction Between Markers and Barriers}

Consideration of barriers is not included in the charge to the panel. The distinction between "markers" and "barriers," however, becomes blurred in cases where message bearing markers cannot be read without taking physical action.

For example, to read a subsurface marker requires digging or plowing at the site, and to read markers buried at the depth of the waste panels requires excavation or drilling. Plowed up markers can be read easily, but fragments flushed up during drilling are so small that inscribed messages are likely to go unnoticed. ${ }^{4}$

Therefore, a marker system on the level of the waste panels should include a component that forces the driller closely to inspect the material being drilled into. Encounter of ultrahard material fragments, or even Thermit ${ }^{5}$ ignited by a mechanism set off by drilling through the enclosing titanium container would achieve this objective.

These "attention getters" have been treated as part of the marker system, as their purpose is not to prevent physical intrusion but to force attention to the markers.

\subsubsection{Five Principles to Maximize Durability}

Longevity of the marker system can be improved by adhering to five basic principles:

(1) Setting up a benign environment:

How long a material lasts is frequently determined more by the environment than by the material's inherent properties. In a benign (i.e., a dry and low humidity) environment even organic materials can survive for long times (papyrus, mummies). On the other hand, even highly corrosion resistant materials are likely to disappear in a wet, "aggressive", environment, as, for example, a hot brine solution.

Therefore, a general principle to ensure marker survival should be to set up a "local" environment beneficial to the marker's survival.

4 Unless the marker is specifically designed for even fragments to attract attention - e.g., through the use of brilliant color.

5 Thermit: a mixture of aluminum powder and iron oxide that when ignited generates a great amount of heat and is used for welding. 
Examples would be locations shielded by berms or sacrificial walls against wind driven erosion and by overhangs against precipitation. For buried markers, archaeological finds in New Mexico can provide guidelines for setting up beneficial environments.

For example, the New Mexico Museum of Natural History in Albuquerque contains a stunningly well preserved skeleton of a young camel that roamed in the Albuquerque area 10,000 to 20,000 years $^{6}$ ago when the climate was wetter than today (see Figs. 4.4-1 to 4.4-4). ${ }^{7}$ This skeleton was found in a bed of sand and gravel in a commercial gravel pit just outside Albuquerque. The skeleton, down to the smallest vertebrae at the tip of the tail, is perfectly conserved. Thus even a material of medium durability ${ }^{8}$ can survive for very long times without losing small features when embedded in a suitable environment (sand and gravel). To duplicate these conditions for buried markers, one of us (DGA) has acquired some data on the Albuquerque site. The site consisted of a mixture of alluvial sand and gravel (one to a few inches thick). The recommended strategy, therefore, is to bury important markers, particularly those in the access shafts (see Section 4.4.9.12) in an appropriate mix of well-drained sand and gravel.

Similarly, guidelines for setting up conditions maximizing the survival of inscriptions on markers can be extracted from Indian rock carvings, even though the age of the oldest of these is about one tenth of the design life of WIPP marker system.

(2) Avoid mixing materials in a single structure.

Bringing different materials into contact opens the possibility for chemical reactions. ${ }^{9}$ In a non-sliding contact, temperature changes will create thermal stresses unless the thermal expansion coefficients are matched. Thus, it is best not to mix different materials in the construction of a marker (e.g., use engravings, not inlays for inscription).

If contact of different material cannot be avoided, as for example at the interface between a concrete foundation and the ground, it is important to minimize the possibility for chemical reactions (e.g., by inserting an impervious clay layer between concrete and soil containing sulfates).

(3) Working with large size components.

For illustrative purposes, let us as assume that a marker weathers at the rate of $1 \mathrm{~mm} / \mathrm{year}$. Although a small value, it will amount to more than 30 feet over the design life of 10,000 years. A structure a few feet in size will disappear but a structure many feet in size will lose

6 The age is in dispute. According to Mike O'Neil, District Paleontologist, U.S. Bureau of Land Management in Santa $\mathrm{Fe}, \mathrm{NM}$, the skeleton might be as old as 80,000 years.

7 One of us (DGA) is indebted to Mike O'Neil, District Paleontologist, U.S. Bureau of Land Management in Santa Fe, NM, for the loan of the original slides of this site.

8 Bone is quite durable in an alkaline environment but will not last in an acidic environment.

9 Although it is possible to test for such reactions, extrapolation from laboratory tests, typically carried out at elevated temperatures, to 10,000 years is inherently uncertain. Thus, it is best to minimize the potential for reactions through a "same material" strategy. 


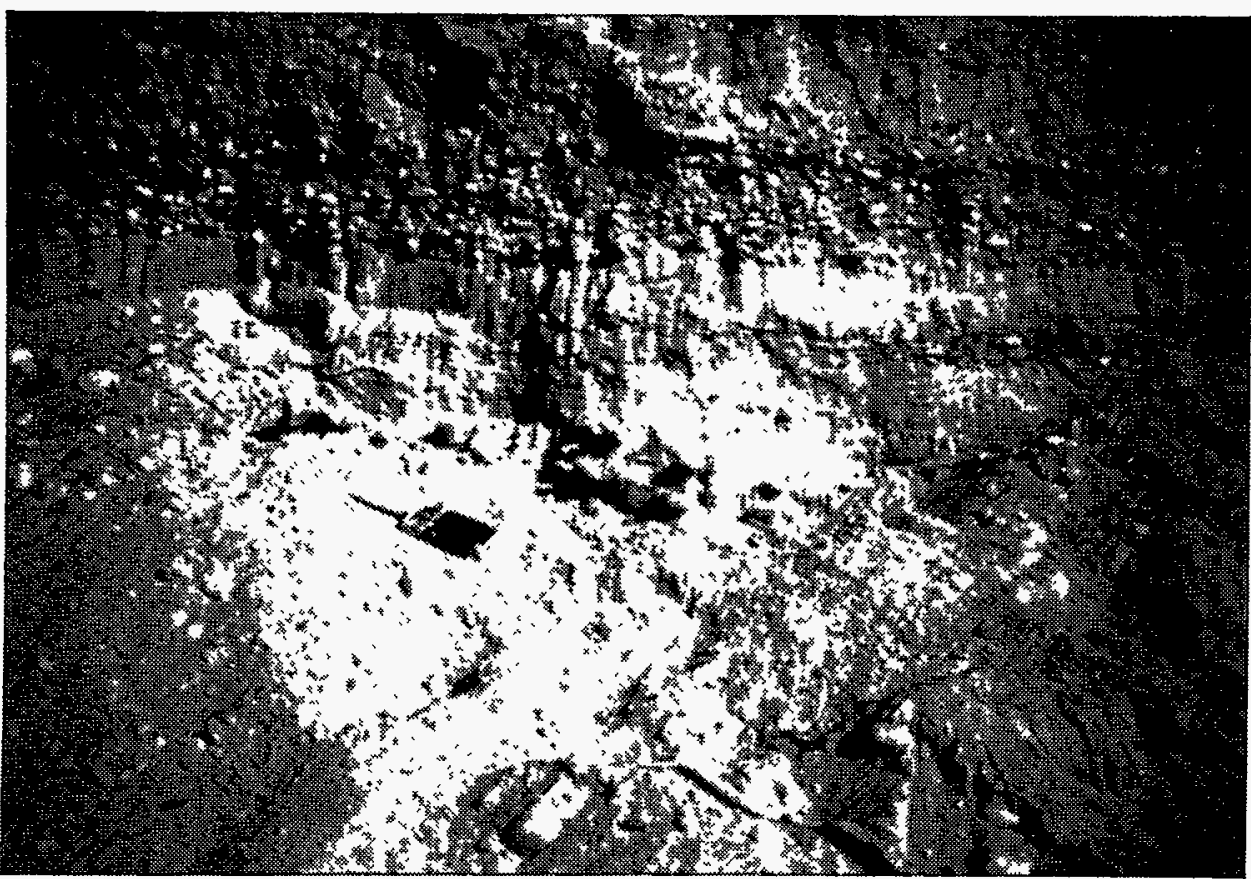

Figure 4.4-1. Camel Skeleton: Initial discovery, cervical vertebra (photo courtesy of Mike O'Neil, Bureau of Land Management).

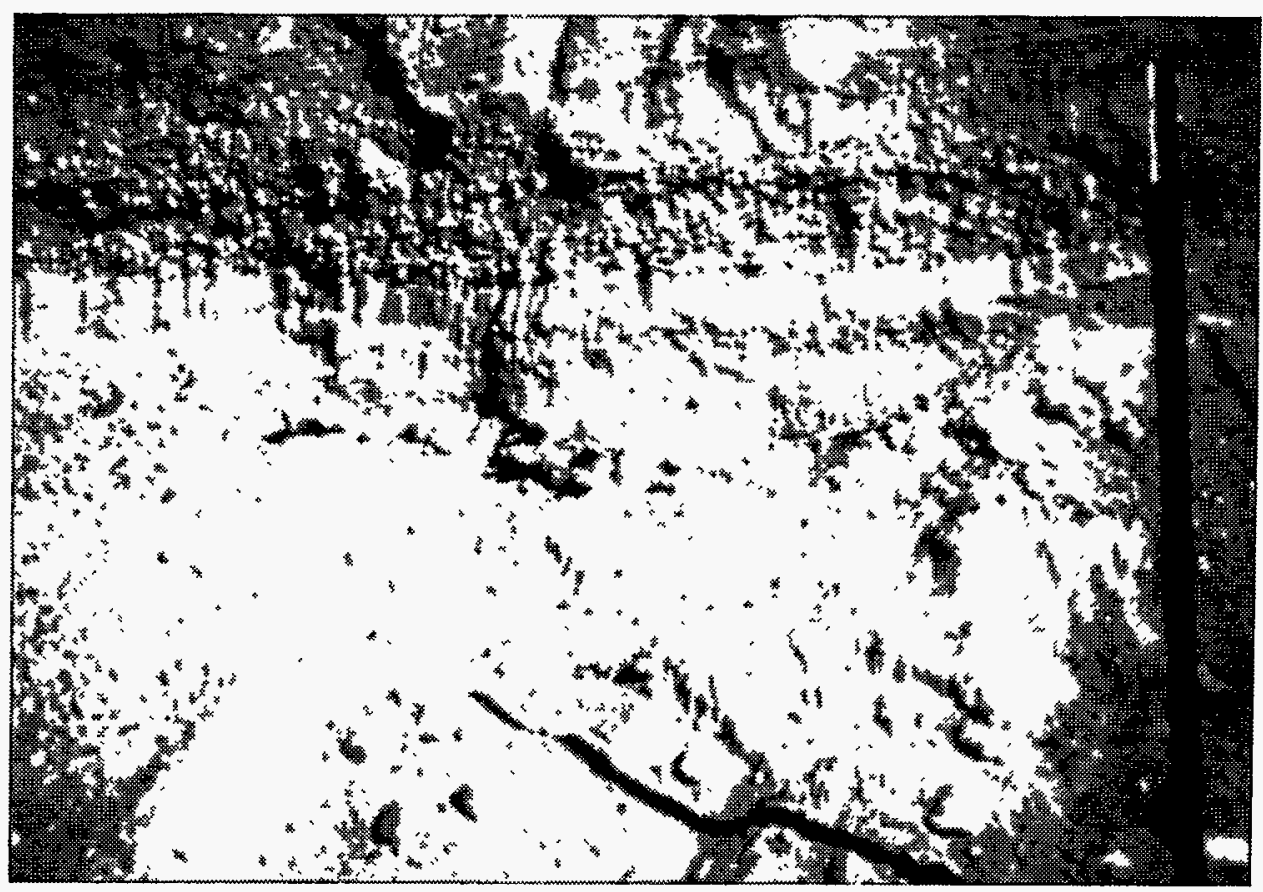

Figure 4.4-2. Camel Skeleton: Pre-excavation (photo courtesy of Mike O'Neil, Bureau of Land Management). 


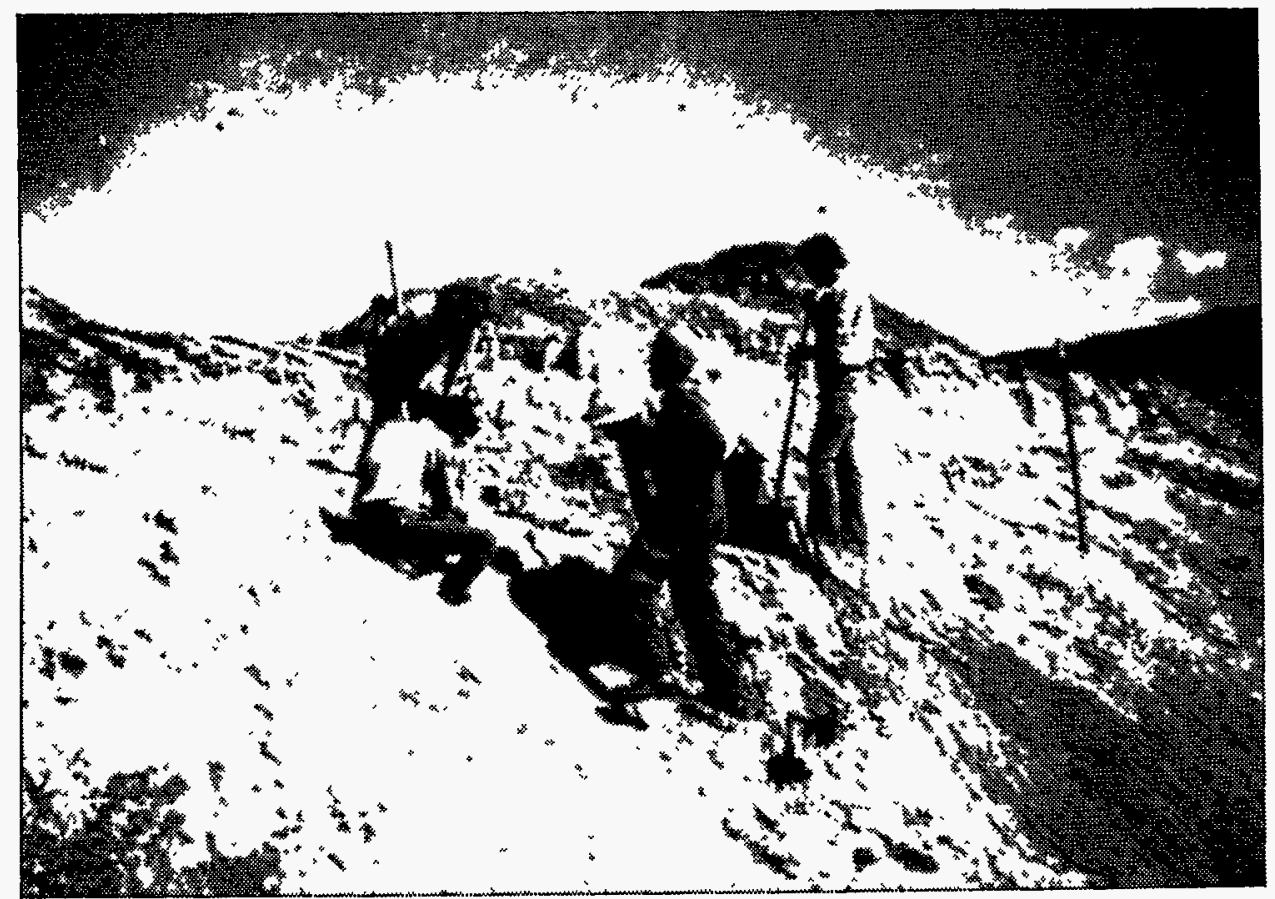

Figure 4.4-3. Camel Skeleton: Overburden removed-excavation begins (photo courtesy of Mike O'Neil, Bureau of Land Management).

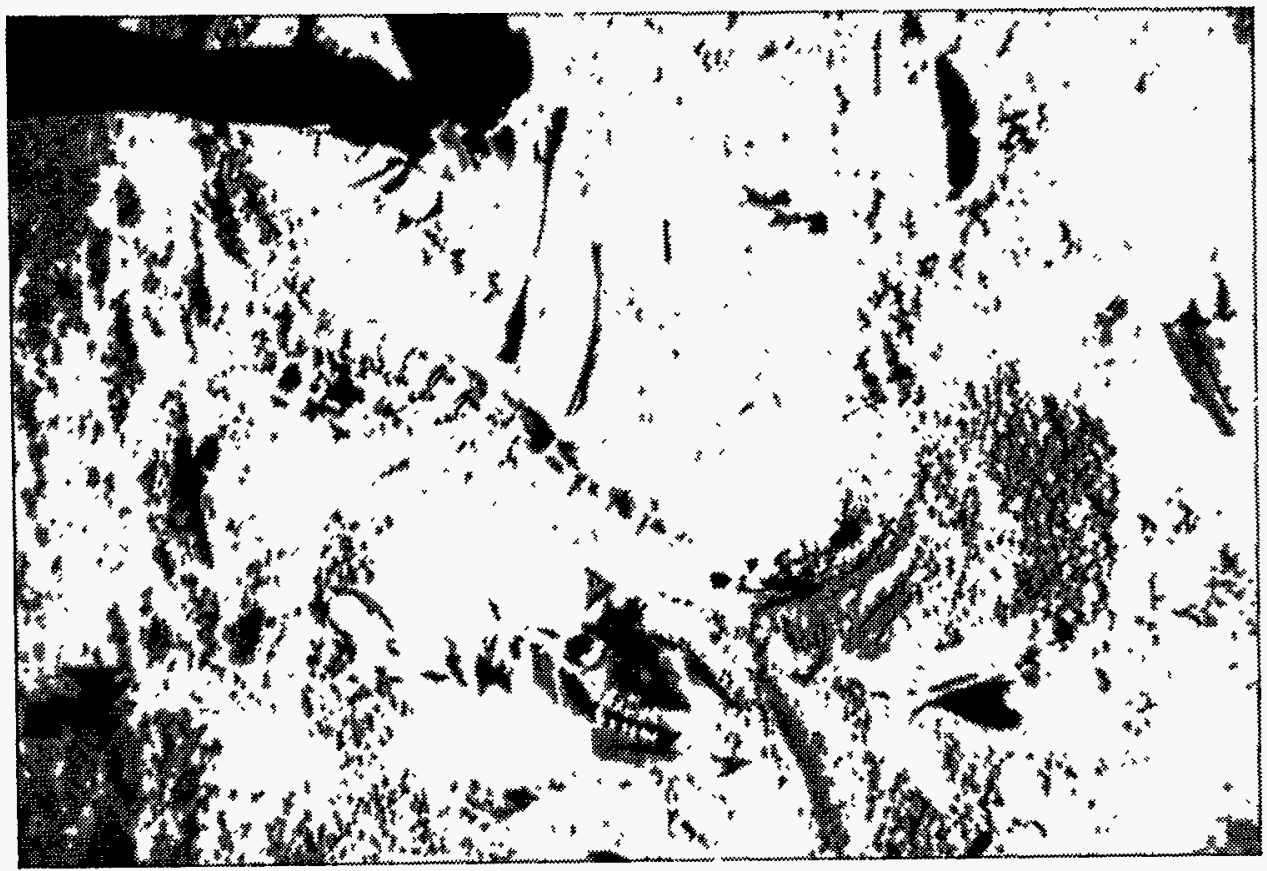

Figure 4.4-4. Camel Skeleton: Camel being exposed-soft sand (photo courtesy of Mike O'Neil, Bureau of Land Management). 
but a small fraction of its volume. Thus size is an important factor in durability. It is no accident that structures that have survived in their original shape (pyramids, Fig. 4.4-5; sphinx, Fig. 4.4-6; monolithic tombs, Figs. 4.4-7, 4.4-8) for long periods of time are very large. (Note, for example, the scale of modern buildings in the background of Fig. 4.4-6 to the sphinx.) Thus, it is important in above-ground structures to work with marker structures of large size.

Because erosion tends to progress linearly in time, ${ }^{10}$ it makes little sense to use a logarithmic time scale in judging a marker system. That is, the probabilistic division into near future (order 100 years) medium future (order 1,000) years and far future (order 10,000 years) has no physical base even though it pleases human perception.

Interestingly, the principle that size promotes durability extends all the way to everyday constructions. Biczók [Ref. 4-2] states that large (on the scale of meters) concrete structures, all other things being equal, are much more resistant to erosion than small ones. Empirical observations over several decades show that the actual corrosion of concrete structures (which for concrete is usually moisture related) is much smaller than expected from laboratory experiments on small specimens (Biczók [Ref. 4-2] cites the example of a mortar sample that had survived in excellent condition in seawater for 17 years, but when enclosed in a barrel filled with the very same seawater completely corroded in 16 days).

\section{(4) Redundancy}

Fourth, because it is difficult to foresee all possible scenarios detrimental to survival of the markers, redundancy must be applied to every physical aspect of the marker system, i.e., to location (above, semi-buried, and below-ground structures), structural design elements (berms, monoliths, rooms), and materials selection.

Monoliths of stone should be made from rocks of granite, basalt, and sandstone; concrete structures from portland-, aluminous-, or ferro-cements; scattered markers from fired alumina, beryllium oxide, earthenware, porcelain, single crystal sapphire (e.g., aluminum oxide discs), different glasses (pyrex, borate), and maybe even metals (titanium, stainless steel).

To preserve a readily perceived pattern, the placement of materials, where possible, should alternate in a sequence (i.e., one monolith of basalt, followed by one of Sandia granite, followed by one of Vermont granite, followed by one of sandstone, etc). Scattered markers made from different materials should be arranged in concentric rings or radial rows.

${ }^{10}$ As long as the amount removed is a small fraction of the original volume and the surface composition and morphology does not change. 


\section{Egypt}

Perhaps the most famous architectural monuments in the world, the pyramids were built of stone with a rubble core as enlarged and elaborated versions of the humble mudbrick mastaba. The masses of masonry were partly symbolic, partly protective and seemingly disproportionate for the sepulchral chambers they sepulchral chambers they
covered. They were worthy memorials but hardly inviolate. A remarkable ability in surveying,

immediately obvious from their sheer bulk and proved by the accuracy of layout and orientation was

necessary for their construc

tion. The great pyramid of Cheops differed from the others in its greater bulk and internal layout. It had three separate chambers while the Chephren pyramid had one chamber with two approaches.

wyth two approaches. Mycerinus' tomb had one
chamber and one entrance.

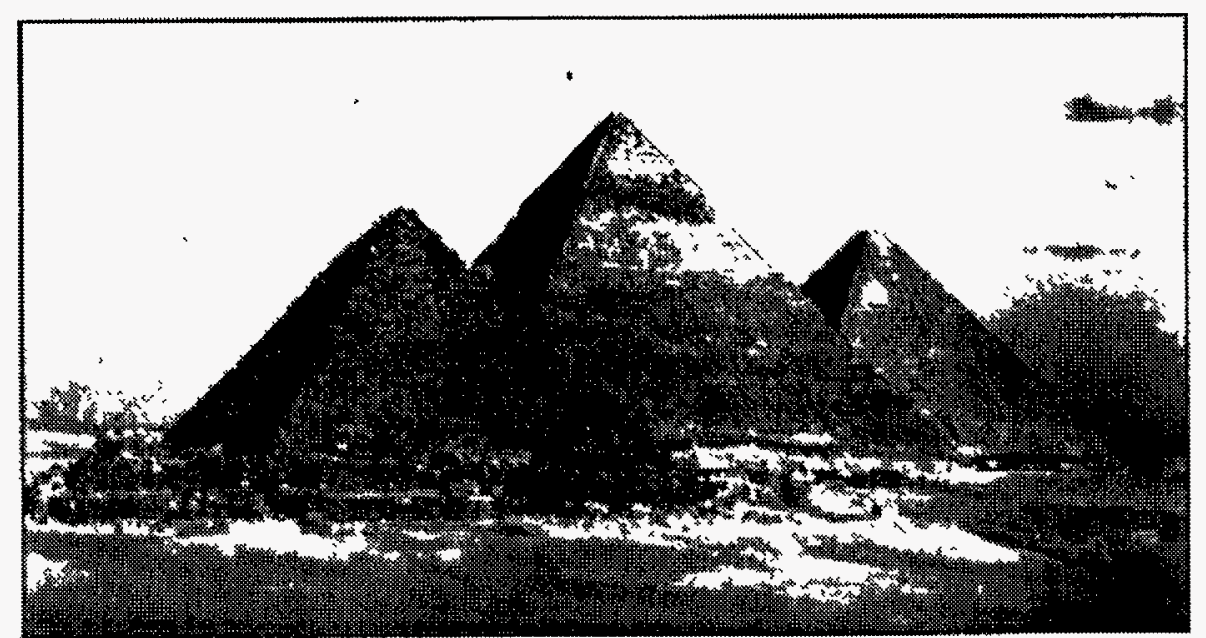

Great pyramids at Giza:Mycerinus, Chephren, Cheops

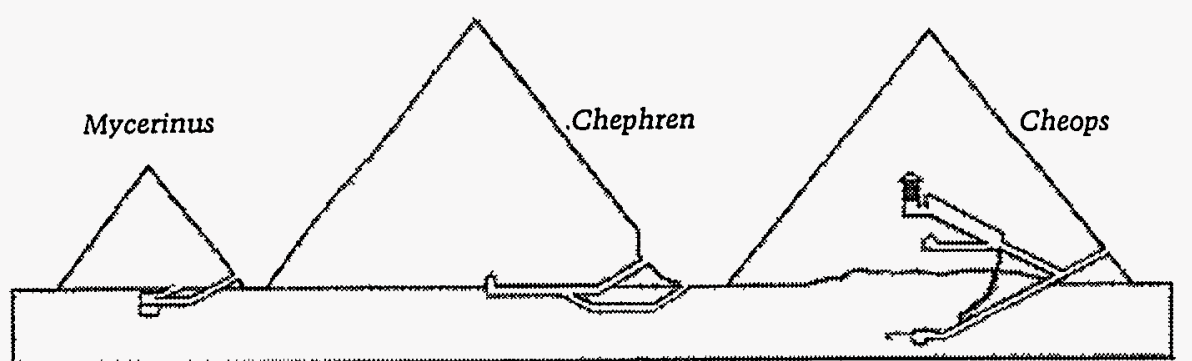

Sections through Giza pyramids showing tombs

Figure 4.4-5. The Great Pyramids at Giza: Mycerinus, Chephren, Cheops. The structures are between 4,000 and 5,000 years old. Copyright (c) 1976 by Jacquetta Hawkes. From The Atlas of Early Man. Reprinted through special arrangement with St. Martin's Press, Inc., New York, NY. 


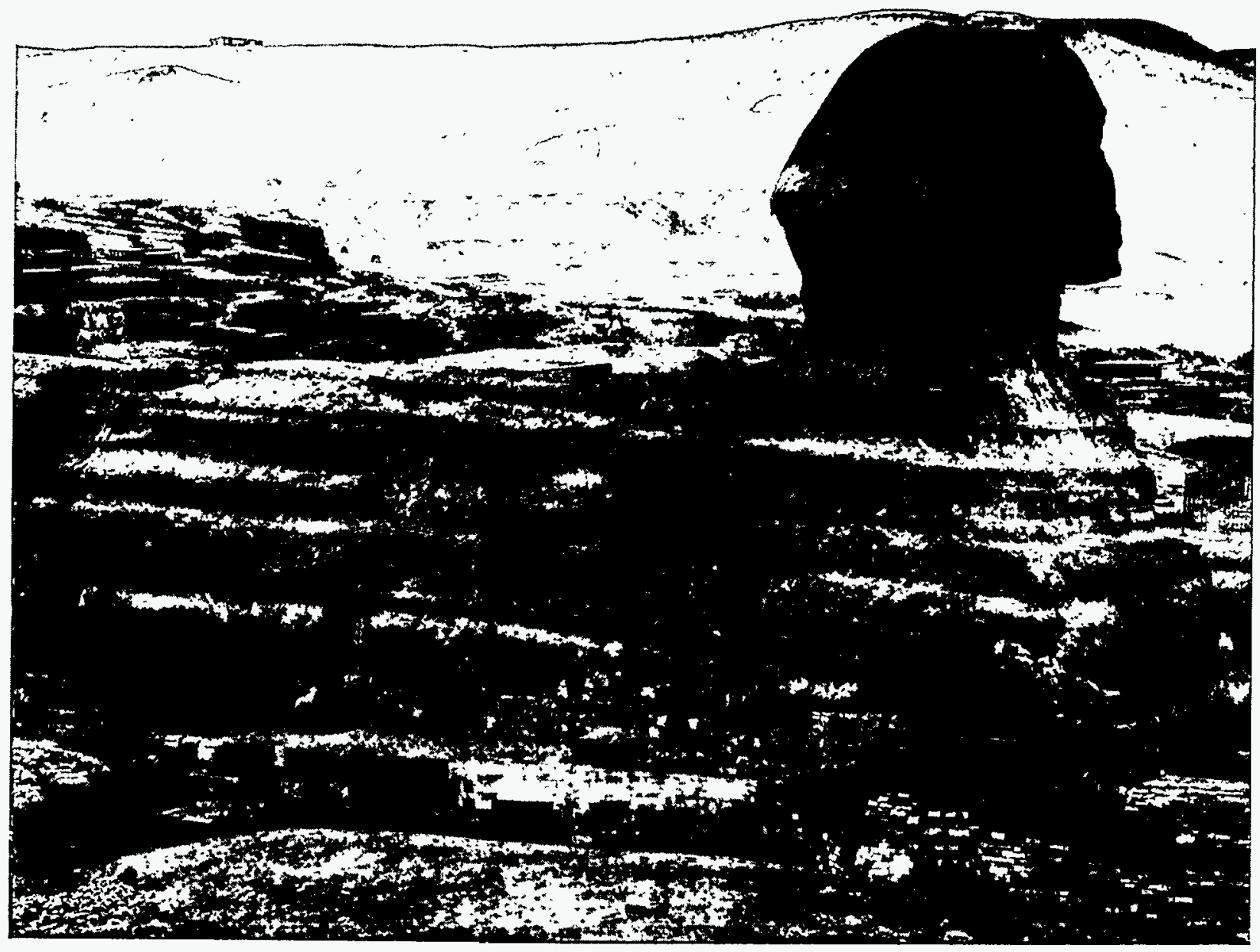

The Great Sphinx at Giza

Figure 4.4-6. The Great Sphinx at Giza. The nose was shot off by troops of Napoleon (vandalism). Its disappearance was not caused by natural forces. Copyright (c) 1976 by Jacquetta Hawkes. From The Atlas of Early Man. Reprinted through special arrangement with St. Martin's Press, Inc., New York, NY. 
Continental Europe

The immense megalithic chamber tombs required great community effort for manipulating blocks of stone weighing up to 100 torrs into position. Some of these chambers contained kerbstones decorated with abstract designs. The West Kennet tomb was built of large boulders and had drystone walls. It was covered by a $350 \mathrm{ft}(106 \mathrm{~m})$ long mound.

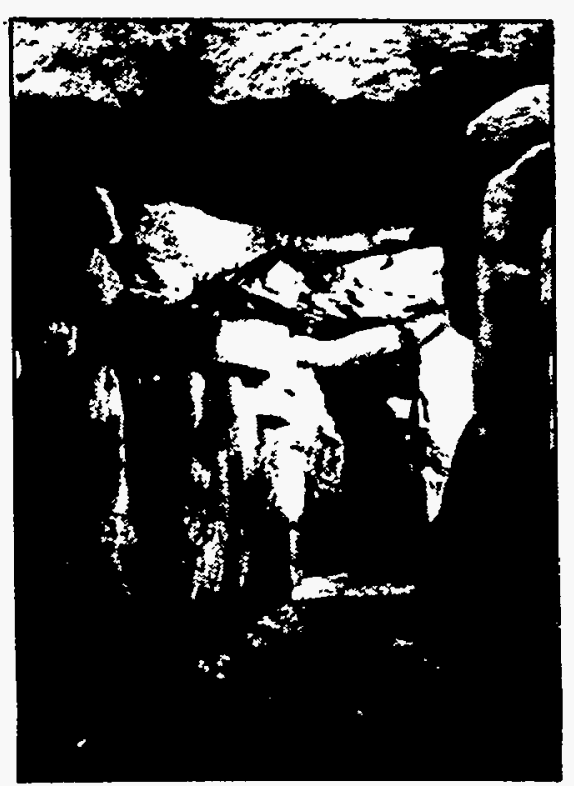

West Kennet long barrow. Wiltshire, England

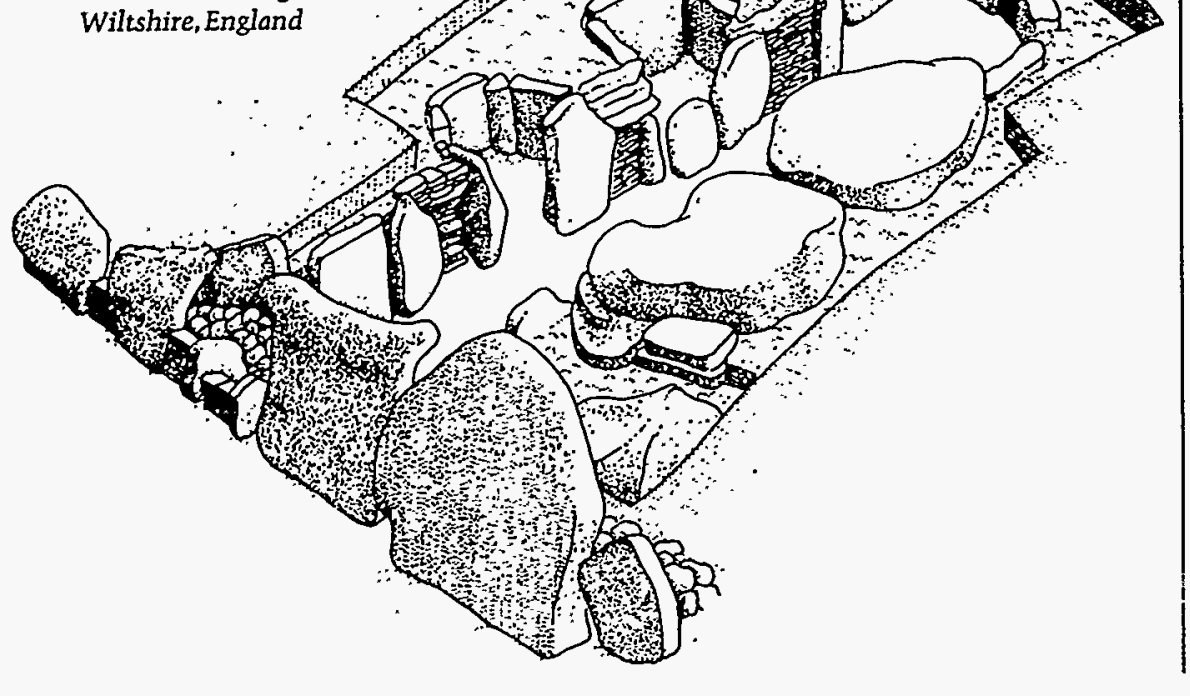

Figure 4.4-7. Monolithic tomb. The one depicted here is in West Kennet, Ireland. Stones up to 100 tons in weight were used in its construction. The grave is between 4,000 and 5,000 years old. Copyright (c) 1976 by Jacquetta Hawks. From The Atlas of Early Man. Reprinted through special arrangement with St. Martin's Press, Inc., New York, NY. 


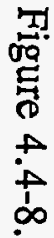

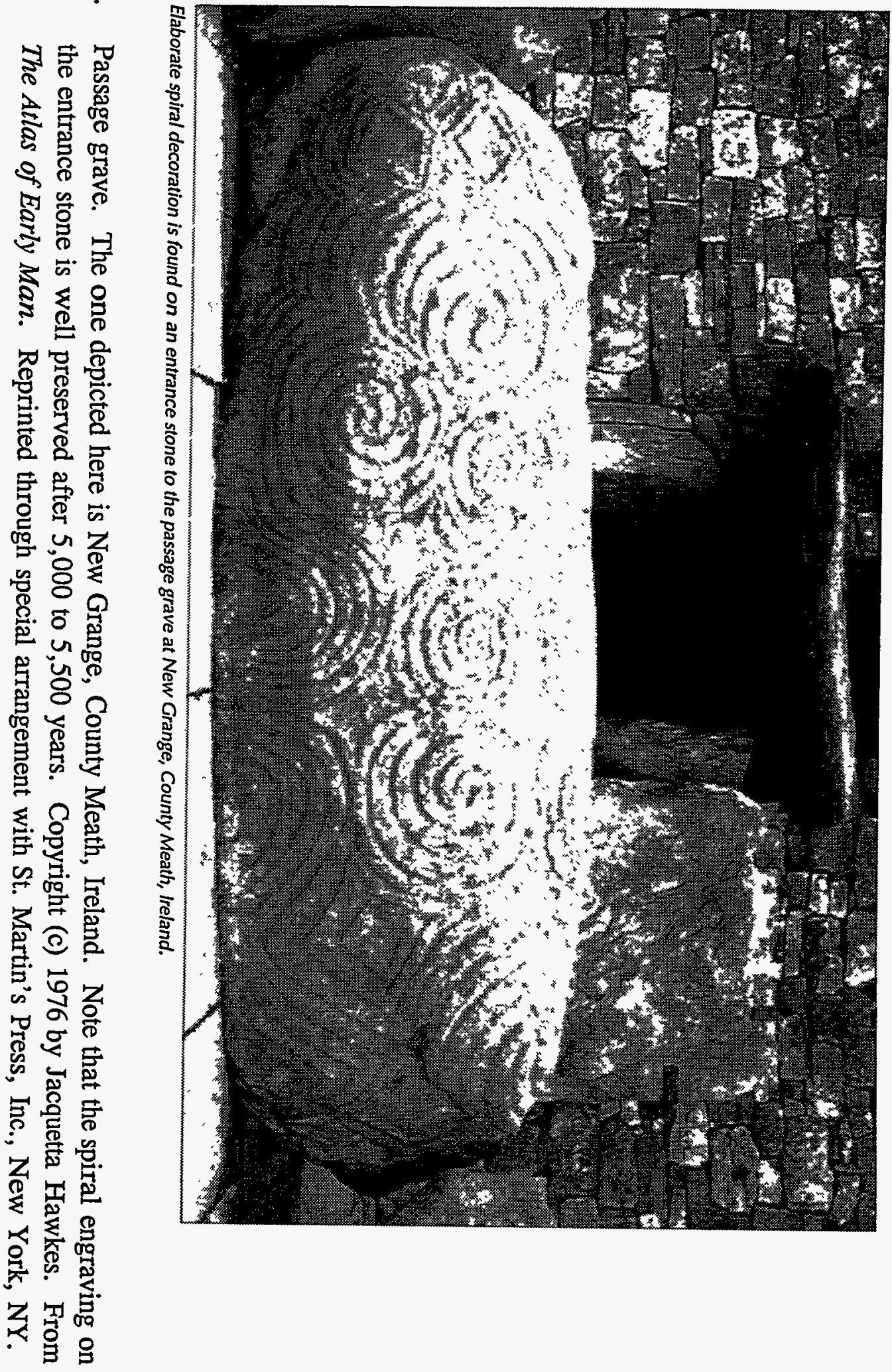


(5) Using on-site testing data in the design

On-site testing of marker materials will allow a better informed choice of materials. This is particularly important for concrete, a material with highly variable properties, see below.

The anticipated operation time for WIPP, 30 to 40 years, is sufficiently long to acquire meaningful upper limits on the erosion rates of materials planned to be used in the marker system.

One of the panel members (DGA) examined the 30-year-old concrete Gnome marker, marking an underground nuclear test explosion located a few miles from the WIPP site. Based on its appearance (but excluding the already visibly eroded water retaining depression on the top) the amount of concrete eroded over that time period appears to be, at most, $1 \mathrm{~mm}$.

If we take this value as an upper limit and extrapolate it to 10,000 years, we obtain $36 \mathrm{~cm}$. As this exceeds the width of the marker, it may disappear.

The above estimate can be criticized from many aspects (e.g., corrosion rates may speed up as the surface becomes less smooth; failure might be of some other mechanism, or from lighting, tornadoes, nuclear explosions, etc.), but it illustrates two important points:

- Data useful to predict erosion of marker structures can be acquired over 30 years, that is, before WIPP is scheduled to close.

- These test data provide guidelines for the minimum feature size of such structures.

The Portland Cement Association, PCA, (Chicago) started in 1940 the long-term testing of concrete at 13 U.S. sites [Ref. 4-3]. Progress Reports are published every 10 years. The test set-up at the WIPP site should use the PCA test set up so that results obtained can be linked to a data base going back to 1940 .

\subsubsection{Environmental Factors}

Site factors influencing survival of markers at the site are climate (temperature cycles and precipitation), wind (tornadoes), wind-blown sand, soil chemistry, surface and ground water, and natural calamities (lightning, earthquakes).

The present climate at the WIPP site is semi-arid. Even if rainfall were to double (which is the worst case prediction for next 10,000 years [Ref. 4-4]), the evaporation rate would still exceed the precipitation rate and the climate would remain favorable to the survival of materials.

A site-specific study of wind and tornado probabilities at the WIPP site has been prepared by Fujita [Ref. 4-5]. This study predicts a $10^{-4} /$ year probability for $119 \mathrm{mph}$ straight line gusts and 
Appendix F: Team A Report

$80 \mathrm{mph}$ tornadoes. The most severe credible tornado that could be expected to occur at the WIPP site, with a probability of $1 \%$ over the next 10,000 years, would have a maximum wind speed of $183 \mathrm{mph}$ and a pressure drop of $0.69 \mathrm{psi}$. To ensure longevity, above-ground markers must be designed to withstand the latter conditions.

Dunes are present at the site and are likely to move, and therefore may bury structures and supply sand for wind-driven erosion [Ref. 4-6]. To ensure continued visibility, above-ground structures must therefore exceed a height of 30 feet.

The panel had no data on the probabilities of earthquakes. An earthquake occurred during the time of this study (Jan. 92). The quake was centered in western Texas/eastern New Mexico and registered 4.6 on the Richter scale [Ref. 4-7].

Because the durability of concrete depends to a very large degree on soil $\mathrm{pH}$, presence or absence of sulfates and chlorides, and contact with the ground water table [Ref. 4-8], on-site data for these parameters must be acquired, should the marker design make use of this material.

4.4.7 Feasibility Demonstration that Durable Markers can be Constructed...if Cost is No Object

\subsubsection{Longevity Principles in the Classical Pyramid Design}

Although guaranteeing survival of structures or markers for 10,000 years appears to be a formidable task, it is straightforward, in the absence of other constraints (e.g., costs, psychological effectiveness), to design a marker system that will be able to transmit engraved and other physically carried messages for ten millennia, provided humans do not disturb the site.

An example would be a $300 \times 300$ feet pyramid, constructed of $9 \times 9 \times 9$ feet (or larger) square blocks of granite. No mortar would be used, and all six sides of each block would be engraved with the full set of Level II and III messages. Thus, should engraving on the exterior surfaces erode with time, future generations would only have to lift one block to uncover a fresh inscription of the same information.

As over $90 \%$ of the Cheops pyramid is still extant after 4,600 years (see Fig. 4.4-5), there is no doubt that such a construction, if left undisturbed, would preserve inscribed messages for 10,000 years.

The pyramid design (Fig. 4.4-9), put forward as an illustration only, incorporates the following design principles:

- Use of all available surfaces to carry messages.

- Redundancy--the message is repeated many times.

- Time evolving messages--as one surface erodes, other, new ones, emerge. 
- Both "exterior" and "interior" storage of messages (the core of the pyramid could be considered as a cave filled with message carrying blocks).

- Easy and obvious access to interior messages.

- All components are made of the same material, eliminating problems that can arise when materials with different thermal expansion and chemical potential are in a non-sliding contact.

- The structure is tapered with a slope less steep than the talus slope. ${ }^{11}$ Even when shaken by a large earthquake, the structure, therefore, would largely retain its original shape.

- The load bearing stress is compressive (i.e., tends to close any crack that might form).

- No tensile surface stresses exist (which would promote crack opening if a crack were to form).

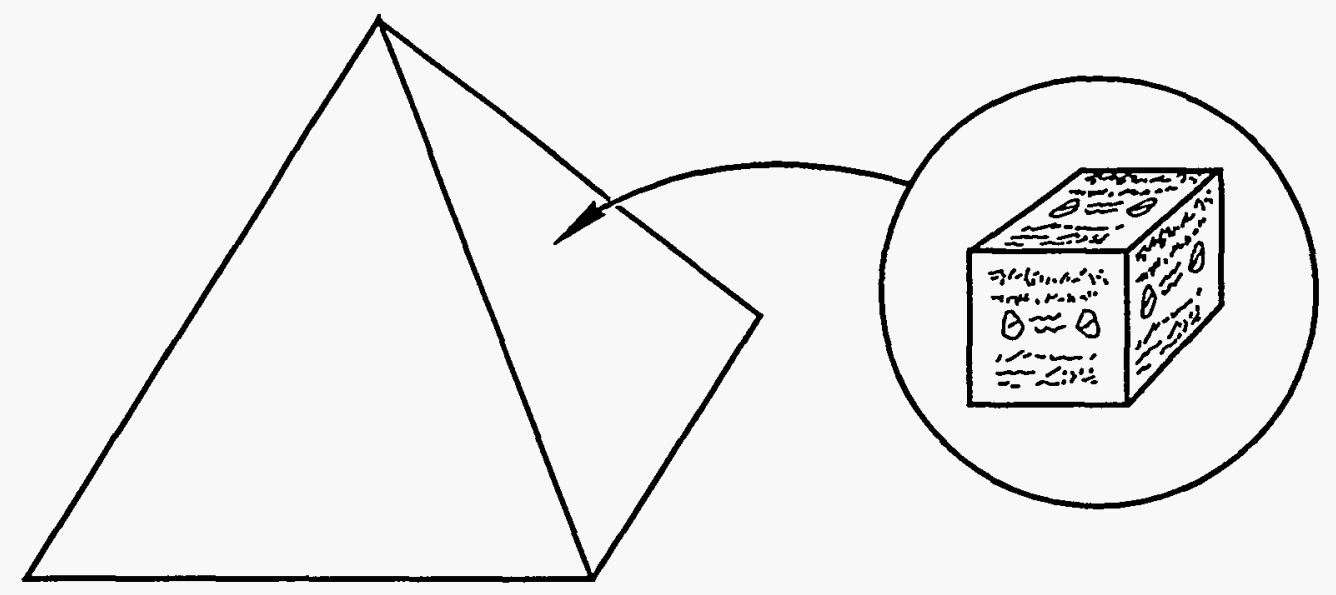

Figure 4.4-9. Pyramid of engraved blocks: An example of durable message transmittal.

The above example also illustrates that a design guaranteed to survive 10,000 years will be expensive. Assume that $9 \times 9 \times 9$ feet granite blocks are to be used and that a single block, including engraving, could be fabricated for $\$ 5,000$. The cost of the material alone, then, would amount to $\$ 62$ million. This would be about $6 \%$ of the to-date cost of WIPP but less than $1 \%$ of the projected cost of WIPP over its operating lifetime.

"The talus slope is the steepest slope a pile of "granular" material will take. 
Appendix F: Team A Report

The high cost of the design is not accidental. Any realistic consideration of proposed marker systems will show that a tradeoff exists between longevity and cost. Any above ground marker system secure against the forces of nature is, by necessity, a large system made out of durable materials, as only such a system can afford the loss of material over time without losing its function.

Therefore, a meaningful probabilistic estimate of the survival of the marker system can only be made if the cost that can be spent on the system is known.

\subsubsection{Shortcomings of a Pyramid Design}

In addition to high cost, a "classical" pyramid design has the following shortcomings:

- The structure is somewhat difficult to see from an altitude of 30,000 feet (aspect ratio 1:100),

- If smaller blocks are used, it is easily climbed, rendering all exterior message bearing surfaces accessible to vandalism,

- The use of quadratic blocks encourages alternative use of the components by future generations ("quarry"), the form of the structure is one often used to mark honored phenomena and may embody an inappropriate message in its form.

Good visibility from the air is highly desirable, as mankind is likely to continue the use of air transportation. Thus, a large population (conceivably even people off-site who make decisions about drilling) can be made aware of the existence of the site by choosing a large design that could easily and unambiguously be identified from the air as a nuclear waste site. This would require a standard large scale design for all nuclear disposal sites. (One solution, which hinges on the continued use of the radiation sign for the next 400 generations, would be earth berms formed into the radiation trefoil sign with a ring of monoliths forming a central circle. Such a design is discussed by Team B. However, the continued use of any cultural icon over 400 generations is uncertain, see Section 3.3.)

\subsubsection{Minimizing Marker Removal by Humans}

Without doubt, the major threat to the survival of markers is human activity, not nature. Metals in historic sites have nearly always disappeared, ${ }^{12}$ and buildings have been used as quarries (note in Fig. 4.4-5 the removal of the more valuable cladding layer ${ }^{13}$ ).

12 An exception appears to be the bronze doors of the Pantheon, still there after 1,800 years.

13 Again, the Pantheon (Fig. 4.4-10) appears to be an exception, possibly because the use of concrete in some of its construction. 


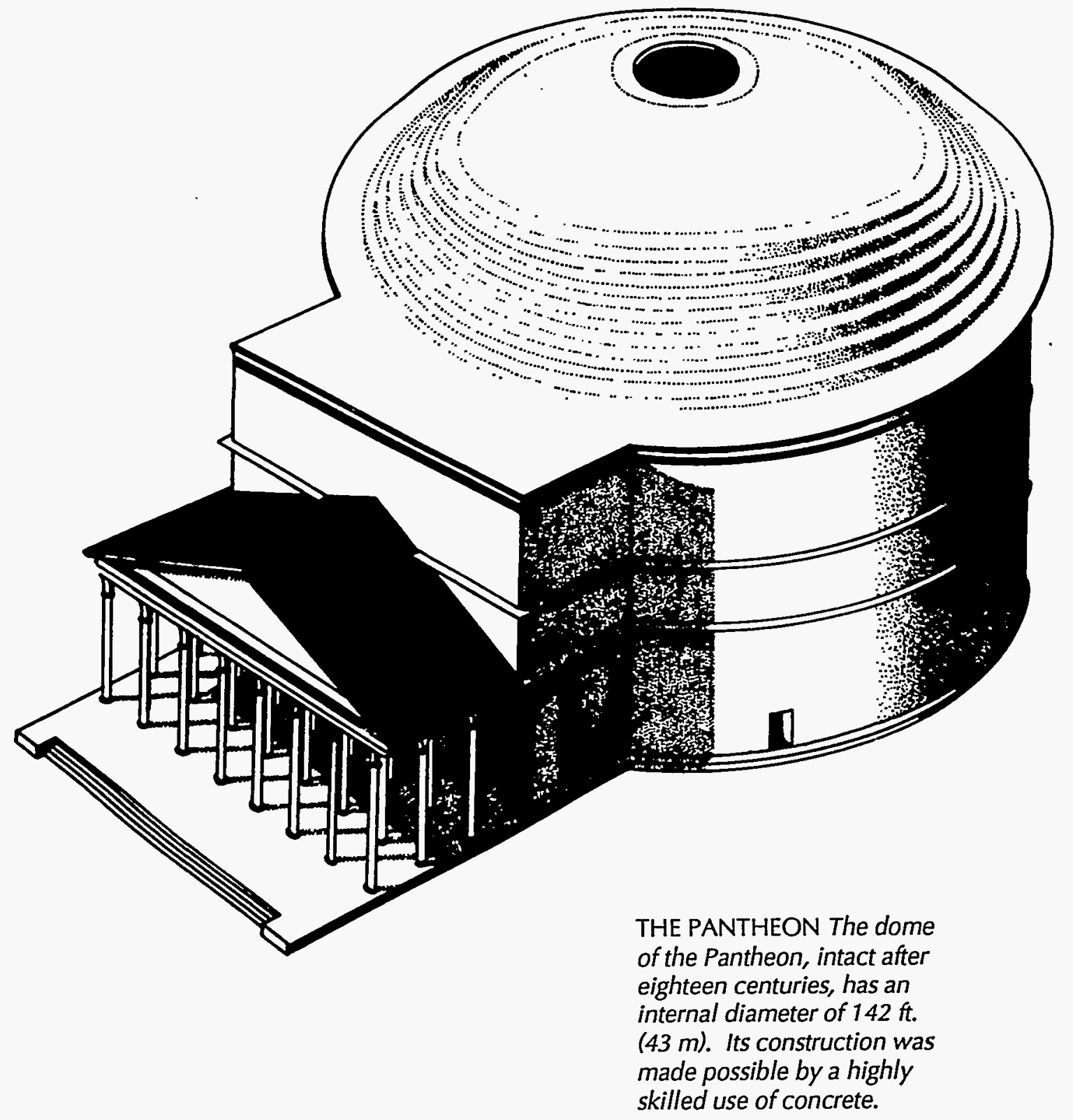

Figure 4.4-10. The Pantheon. This building, which uses, in part, concrete still stands after 1,800 years. Copyright (c) 1976 by Jacquetta Hawkes. From The Atlas of Early Man. Reprinted through special arrangement with St. Martins Press, Inc., New York, NY. 
Appendix F: Team A Report

Vandalism of monuments has been a problem, even when the site commemorates a revered personality, as in the Washington Monument [Ref. 4-9]. ${ }^{14}$

For this reason we recommend the construction of closed Level IV rooms.

Finally, as discussed before, an organized, large scale activity by future societies to remove the marker system is conceivable.

General guidelines to minimize removal of above-ground marker structures are:

- Use of inexpensive materials. Examples are materials found at the site (e.g., caliche) and to a degree concrete.

- Use of otherwise not easily usable materials and building blocks. Examples are brittle materials prone to break when reshaped and large blocks shaped in odd forms. By odd forms we mean specifically forms that can not readily be assembled to fill space (like rectangular block). For example, equal sized blocks of five fold symmetry cannot fill space continuously. ${ }^{15}$

Further to discourage alternative use of irregular shaped components, each component should be shaped such that it cannot rest without rocking or falling over on a flat surface.

- Using interlocking structures involving irregularly shaped blocks that can be assembled into one form only. A computer code to arrive at such a design should take into account:

* Generation of flat surfaces of minimum area to hold desired messages (this would apply only to structures designed to hold time released messages).

* Design of components that cannot rest stably on a flat surface (to discourage alternative use as a weight).

* Self-locking assembly into final form.

* Stability against ground motions caused by large earthquakes.

14 After a construction that spanned half a century, the Washington Monument was opened to the public in 1886. Vandalism immediately became a serious problem threatening "if not curbed, the existence of the monument itself' (Casey, the last of the builders, in 1886)...Guards were hired, and Congress, in 1887 passed legislation forbidding people "dots to chip off fragments or pieces from any of the stone, iron, or other parts of the completed structure...." Violations would be punished by a fine of at least $\$ 5$, imprisonment of 15 days or both. If damage exceeded $\$ 100$, the offender would be remanded for trial and, if found guilty, imprisoned for 6 months to five years. In spite of this legislation, vandalism continued and eventually forced the closing of the interior stairwell of the monument, see [Ref. 4-9] for further details.

15 Twofold object of two different sizes, however, as Penrose showed, can fill space continuously. Such a "Penrose tiling" might be useful in the design of marker structures. 
* Drainage of any water that may enter outside surfaces.

- Use of obnoxious materials

Obnoxious materials are not likely to be removed. However, materials obnoxious in the sense of "bad smell" cannot be durable since they continuously evaporate the small fragments reaching us. Thus, they must disappear with time. However, it might be possible to enclose such materials into long-lasting glass capsules that break when stressed. The glass of choice would be lanthiumborate. ${ }^{16}$

Another long-lasting obnoxious material is a material with a low level of radioactivity. Thus, one could consider the display of small amounts of clearly marked quantities of low-level radioactive materials with long lifetimes (e.g., low-grade uranium containing ores). ${ }^{17}$

- Maximizing the labor component, minimizing material value. An example would be the construction of a very large earth dam from material at the site.

- Maximizing the ratio of work required to remove the structure to that required to make the structure.

This principle strongly favors the use of reinforced concrete, as structures made of such material, when large, are very difficult to remove, while the work for construction is relatively modest. This is very different from a design made out of shaped blocks of stone that requires much more work to make than to disassemble.

The concrete bunkers of the Maginot and Siegfried lines, therefore, have not been removed, in spite of intense political pressure from the local communities, whereas much of the building stones of the colosseum in Rome have been recycled.

- Using above-ground components that are large and heavy (say with minimum dimensions of $6 \times 6 \times 6$ feet, or a minimum weight of 18 tons). Such weights are difficult to remove with farm equipment ${ }^{18}$ but easily handled by today's cranes.

The builders of Stonehenge (1500 BC) used blocks of up to 54 tons in weight (Fig. 4.4-11). The builders of the West Kennet tomb (3000 - 2000 BC) used blocks of stones weighing up to 100 tons (Fig. 4.4-7) [Ref. 4-10].

16 One of us (DGA) would like to thank Dr. Morse, of Corning Glass Laboratory, for several valuable discussions.

17 Contrary to public perception low-level, radioactive materials are used widely in specialized applications. For example, many thousands of armor piercing rounds made from depleted uranium were used by U.S. forces in the war following Iraq's invasion of Kuwait. It was the low-level radioactivity imparted on the hit target that permitted the unambiguous identification of friendly fire.

${ }^{18}$ It is unlikely that farm equipment changes much in terms of load capacity. 


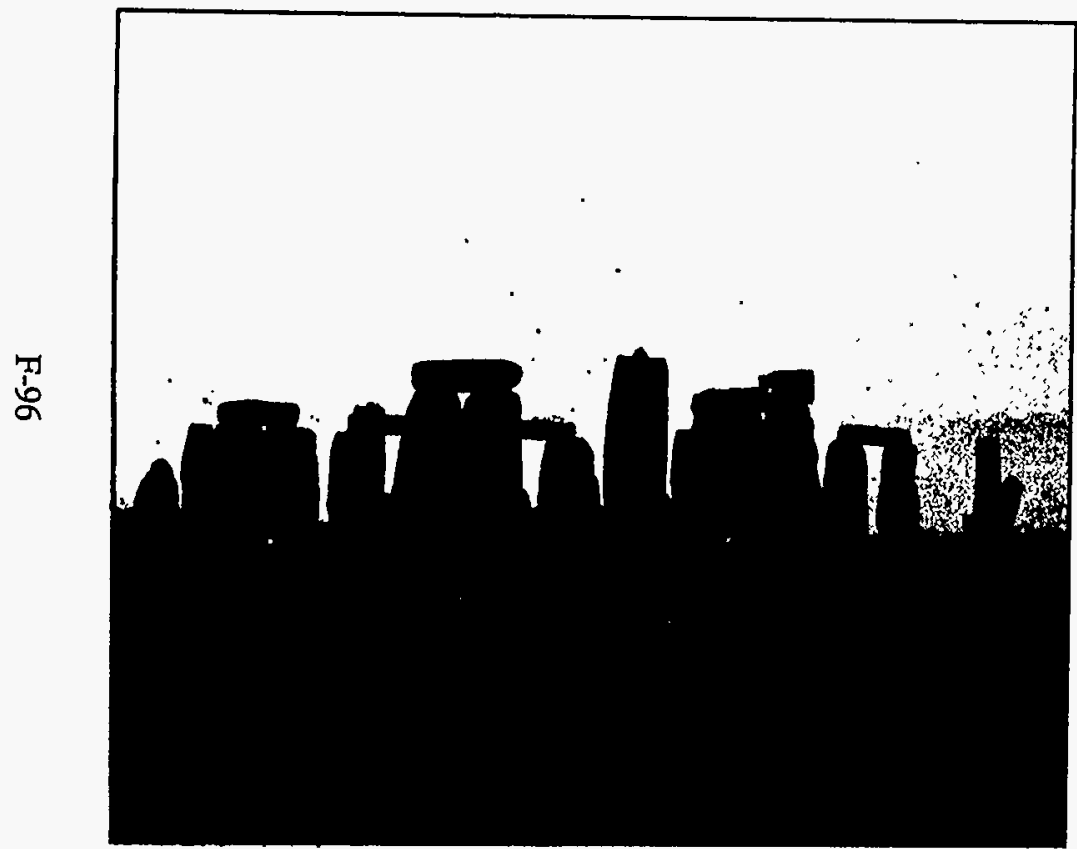

Stonehenge tòday
Continental Europe

Stonehenge is outstanding among the henge monuments in Britain - circular structures of stone or wooden uprights. Blocks of stone up to 54 tons were transported to this site from 24 miles ( $40 \mathrm{~km}$ ) off dressed to shape and erected. Others were raised to serve as lintels. The shaping included subtle architectural tricks like the swelling of uprights, the tapering of lintels, and the curvature of the circle of lintels over the outer ring all intended to improve the visual, aesthetic appearance of the building. Within the main structure were subsidiary stones of

around 4 tons weight.

These were imported with immense labour from Prescelly, 132 miles

$(220 \mathrm{~km})$ away in Wales.

The site appears to

represent the corporate achievement of a wealthy society, whose leading members lay in the barrows which cluster around. (The burial illustrated is of a similar but less architectur-

ally inclined group in Germany.|Two major controversies have raged coner this monumentiged buildings functiont. The buildings function has been strongly debated and questionable contact with Mycenaean Greece has been inferred from the architectural niceties.

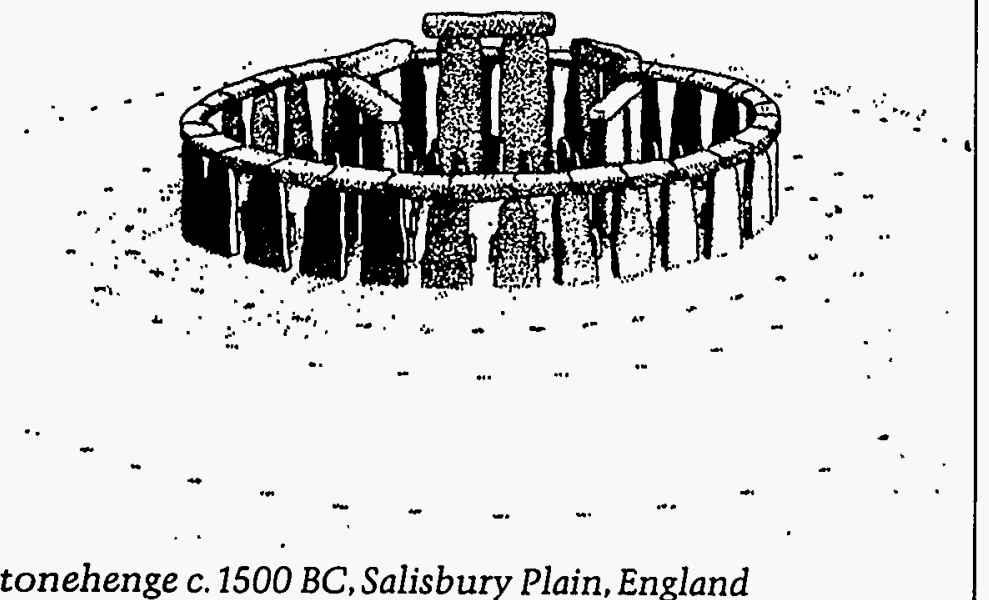

Figure 4.4-11. Stonehenge. Blocks up to 54 tons in weight were transported 24 miles away to construct this monument, much of which stands after 3,500 years in spite of relative shallow embedding of the monoliths. Copyright (c) 1976 by Jacquetta Hawkes. From The Atlas of Early Man. Reprinted through special arrangement with St. Martin's Press, Inc., New York, NY. 
For subsurface and scattered markers, the following factors minimize the probability of removal of markers:

- Distribution over a wide area and a variety of depths.

- Use of materials that are not easily retrievable, i.e., materials with physical characteristics (density, dielectric and magnetic constants) similar to that in which they are embedded. This will make it difficult to use automated retrieval.

- Use of inexpensive materials.

Examples incorporating these guidelines can be found in Section 4.3.

\subsubsection{Durability of Some Common Elements of a Marker System}

\subsubsection{Introduction}

In this section, we discuss the durability of structures considered in this report. These structures are examples only, and are not meant to indicate the design of the site, which is an architectural decision.

The durability of these structures depends on their environment, their design, and the materials used. Because these factors are interrelated, materials are discussed as they would be used in generic marker elements.

Above-ground elements include the following:

- Earth berms,

- Monoliths,

- Structures generating tones or noises when blown on by wind, and

- Enclosed rooms housing Level IV messages.

Below-ground structures comprise the following:

- Subterranean markers (scattered surface markers, markers denoting shafts, markers at the depth levels of waste panels), and

- Buried magnets, electric, and seismic resonant structures.

These elements will be discussed in the above order. 


\subsubsection{Large Earth Berms}

Earth berms have been used as barriers (e.g., in fortifications) and to define areas (e.g., in formal gardens). Based on the historic record (prehistoric mounds, Roman lines, ${ }^{19}$ Hadrian's wall) the probability that large earth dams survive for 10,000 years is very high.

They should be made out of material found at the WIPP site to minimize their material value and should be designed such that their removal requires much work.

The earth berms should be covered with caliche (white) that will (at least temporarily) increase contrast with the environment (light brown). This contrast will increase visibility of the site from the air. Consideration should be given to the size and orientation of shadows thrown by the berms so as to maximize visibility (and, possibly, to generate forms of artistic interest).

To avoid the possibility that significant sections get buried by migrating dunes (estimated to reach heights of 30 feet), the minimum height of these earth berms should be 50 feet. This requirement together with the talus slope, determines the minimum lateral extension. Except for this constraint, the longitudinal extensions are matters of design.

The area outlined should coincide with the lateral extension of radionuclides at the storage level. That is, with the waste panels plus the upper limit calculated for the lateral movement of radionuclides by appropriate transport mechanisms (diffusion, percolation, and convection).

Earthworks do not provide surfaces suitable to carry message bearing inscriptions, but their construction should be combined with the following:

- Interment of scattered markers whenever possible.

- Interment of conductors to mark the site by man-made changes in soil conductivity.

- Interment of magnetic markers, to mark site by magnetic anomalies.

- Construction of sealed and buried rooms containing Level IV information (see Sections 4.4.9.9 and 4.4.9.10 for details).

\subsubsection{Monoliths Made of Stone}

Monoliths made out of natural stone have survived for 3,500 years at Stonehenge, quite a wet climate. At the WIPP site, monoliths are very likely to survive at the site for 10,000 years if bedded properly and left undisturbed.

Monoliths (and walls formed of monoliths) are suitable carriers for Level II and III information. To minimize the probability that the inscribed information will be destroyed by acts of vandalism, a monolith must have a height such that at least one set of these messages is not

19 The fortification line between the Roman Empire and the Teutonic tribes, stretching from the Danube to the Rhine. Even though the climate is rather wet, resulting in the loss of all wooden fortifications, the earth berm itself survives. 
accessible to a standing person, or a person on horseback or standing on top of common farm equipment (wagons, pickup trucks, tractors). One of the areas left for future re-inscription, as well, should be outside the reach of such persons.

Level II and III messages should be inscribed several times over the length of the monolith including inscriptions below ground. The topography of the engraved messages must make it clear that messages continue below ground (deeply engraved spiral band with alternating inscriptions and empty spaces for re-inscription?). Thus, if the inscriptions above ground should weather away, digging would unearth a fresh set of inscriptions.

These monoliths should

- Where feasible, be protected from wind-driven sand that can cause erosion by other design elements, e.g., on the lee side of berms or by encircling "sacrificial" walls. Their heights could vary but should not be less than 4 feet. Openings in the wall should be narrow and on the lee side of the prevailing wind.

These walls could be constructed of concrete, or of irregular, tightly interlocking stones and, if suitable, may carry Level II messages on its inside.

- To ensure a sound foundation, the buried part of a monolith should be equal to or greater than its exposed part or it should be imbedded in the rock strata below. Monoliths should be placed upright, or nearly upright to avoid the formation of tensile stresses at the surface.

- The construction material of monoliths should be natural stone with a minimum compressive strength of 25,000 psi. Suitable materials are granite (average strength $26,000 \mathrm{psi}$ ) and basalt $(29,000 \mathrm{psi})$. The principle of redundancy favors use of a variety of materials.

- Some rocks, for example felsite (47,000 psi) are notably stronger, but we found no information on their availability. Granite can be found in the Sandia mountain range.

- The erosion of rocks by sand as measured by the abrasion test used for concrete (aggregate particles between $1 / 2$ and $3 / 8$ inch in diameter subjected to 500 abrasions with Leighton Buzzard sand, see Neville [Ref. 4-8]) is similar and ranges from 16.5 for limestone to 19.2 for flint (the higher numbers being worse). Thus, in regard to erosion by wind-driven sand, there is no compelling reason to prefer one natural rock material over another.

Removing the constraint of uprightness from monoliths, e.g., using diagonally inclined monoliths, considerably increases design options to mark the WIPP site (for examples of such designs, see Figs. 4.3-1, 4.3-3, and 4.3-7).

However, such structures will develop tensile stresses at their surface that increase with their deviation from the vertical. Tensile stresses, in a brittle material, can lead to catastrophic failure once a crack, however slowly growing, reaches a critical length.

In structures designed to last, tensile stresses therefore are undesirable. It is recommended that only a subset of monoliths be positioned in such a way and that their inclination be limited to 
angles that keep the magnitude of the tensile stress at the surface below 0.6 of the compressive strength of the material used. This recommendation, incorporating a safety factor of 10 , is based on the observation that the strength of stone (or non-reinforced concrete) in static tension is about one-eighth of the strength in compression and that the fatigue strength is about half of the static strength.

The foundation of inclined monoliths must be such that the center of gravity coincides with the center of the foundation footprint.

\subsubsection{Concrete Monoliths}

The probability of survival of monoliths made out of concrete has been looked into by the team in considerable detail, because concrete has several advantages, notably a low price and "in-situ" staying power, the work to remove or recycle heavily reinforced concrete being exceptionally large. More details on concrete can be found later in the various discussions of rooms.

If used as a construction material, only a subset of monoliths should be fabricated with concrete, and the overall design should take into account that this subset may disappear after 2,000 to 5,000 years (see below).

To ensure survival for that (and possibly even a longer) time period, the following practices must be adhered to:

- High quality, 20,000 psi, concrete is to be used.

- Sacrificial walls are to be installed at the base of the concrete monoliths to provide protection against wind-driven erosion.

- The monolith is to be sized and designed to remain stable and upright even if eroded (possibly unsymmetrically resulting in an unbalance) to such an extent that the loss of material may reach the order of tens of centimeters, with the exact value to be determined by the outcome of test results at the site.

- Soil at the foundation is to be checked for sulfates and chlorides, and, if necessary, an intermediate bed of clay or other impervious material is to be inserted to separate the concrete footing from the soil.

- The footing is to be in a well-drained stratum.

The protection of steel reinforced concrete monoliths against lightning is a subject of further research. 


\subsubsection{Composite "Monoliths"}

Structures similar to monoliths, but consisting of a concrete core and a rock cladding are cheaper than stone monoliths. They are conceivably durable if they are designed with care, especially against the intrusion of water, and constructed without mortar bonding to allow movement of the core relative to the cladding.

The thermal expansion coefficient of rocks and concrete is similar. Thus, provided both materials are at the same temperature, thermal expansion differences between core and cladding are small. For example, in a 30-foot-long "monolith" subjected to an $80^{\circ} \mathrm{F}$ temperature swing, the difference in thermal expansion between core (concrete) and skin (rock) would be below $1 \mathrm{~mm}$. If this difference would be accommodated by homogeneous elastic deformation, the corresponding stress would be a few psi.

The above result is based on the assumption that the core and cladding, at any point in time, have the same temperature; but that is not likely because in the morning sunlight first heats the surface and then the interior.

Assume, for example, that the surface cladding has reached a temperature of $55^{\circ} \mathrm{F}$, but the core remains at $30^{\circ} \mathrm{F}$. Such a temperature profile could occur when a cold night is followed by a sunny day. In such a case, the difference in strain would increase to $1.5 \times 10^{-4}$ and the thermal stress, tensile in nature, on the core would increase to roughly $1000 \mathrm{psi}$. This is $1 / 20$ of the yield stress in compression of good concrete and high enough to cause concern as the fracture stress in tension is about one order of magnitude lower. Thus, a design should be chosen that permits the cladding to move respective to the core, if the latter is fabricated from non-reinforced concrete.

\subsubsection{Markers Generating Noise or Tones}

Audible markers can be fabricated with structures that contain "tuned" air masses that vibrate when set in oscillation by wind.

Both dissonant and consonant sets of harmonies could be generated. Because the only moving component is air, a 10,000-year survival of properly designed structures appears feasible.

\subsubsection{Other Self-Energized Marker Systems}

A team member (DGA) has considered the use of other active markers. However, none of them is likely to survive for more than a few hundred years. Of those, the most durable appears to be thermo-electric power based on the temperature difference between surface and 100 feet below. Such a power source could drive low-power active electromagnetic warning systems. We note that electronic components with exceptional reliability have been developed for use in undersea cables. Devices constructed with such technologies could conceivably survive several hundred years or even more. 


\subsubsection{Above-Ground, Closed Rooms}

The team recommends closed rooms for the Level IV message. To ensure the long-term preservation of the message, it should be inscribed on both the (visible) front of the wall panels fabricated from hard rock as well as on their backs. Removal of these panels (stripping the wall) should expose a second set of identical panels and removal of those the building's walls of stone. (The blocks making up this wall, again, may contain Level IV information, if necessary in a condensed version. As a further backup, removal of a block could make visible further engraved blocks.)

The periodic table of the elements should be made of stone also. It should be large and contain samples of the elements where feasible. Inexpensive materials should be inserted as plugs. Expensive but durable materials, such as gold, should be applied as very thin layers (rub-on or sputtering) to minimize the incentive for removal.

If the periodic table is mounted on the wall, the down arrows towards the radioactive elements stored below should be engraved. If the table is horizontal, down pointing arrows made of stone should be inserted into the table. In each case, the arrow length should give some indication of the total amount of the element stored below.

An above-ground storage site must deal with daily temperature fluctuations that may reach $80^{\circ} \mathrm{F}$. The thermal stresses and movements induced by thermal expansion are detrimental to the longterm survival of the structure and the messages contained therein.

Any above-ground structure for a Level IV message should, therefore, be designed to allow for thermal expansion and to be sufficiently massive to dampen the daily temperature variations. An approximately constant temperature at the actual site at which the information is stored is desirable. For the same reason, direct sunlight on the inscription should be avoided.

To ensure longevity, the building material for any above-ground Level IV storage site should be natural stone. The uncertainty of the durability of concrete rules out its use for crucial above-ground structures.

Based on the historical record, a building constructed with irregular, interlocking natural stones weighing tens of tons should survive for 10,000 years at the site. (Note that megalithic chamber tombs, surviving intact to date (see Figs. 4.4-7 and 4.4-8) were constructed with blocks up to 100 tons in weight.)

\subsubsection{Partially Buried, Closed Rooms}

A partially buried structure is exposed to much smaller daily temperature oscillations. It is therefore much more suitable for concrete construction, which, if properly sized, is reasonably 
likely to survive for 10,000 years, provided the foundations remain above the water table for the design period.

Thus, buried Level IV rooms may be constructed of concrete, if the structures are covered with earth and if the minimum dimension anywhere in the structure is several feet. We note that a design incorporating similar principles has been proposed for the long-term storage of transuranic waste [Ref. 4-11].

Because this is the first time at which the team recommends the use of concrete for the construction of a component with a design life of 10,000 years, a more detailed discussion of the durability of concrete is in order.

Mankind has experimented with stone for over 35,000 years, and 5,000-year old tombs are still in fine condition (see Fig. 4.4-8 taken from [Ref. 4-10]). Mankind's experience with concrete is limited to 2,000 years. Although some 2,000-year-old concrete structures have endured to this date, e.g., Fig. 4.4-10 (not to mention Roman bridges-e.g., 6 of the 8 built by the Romans across the Tiber are still in service), this is an insufficient base to predict survival for 10,000 years. Furthermore, contrary to expectation, it is conceivable that Roman concrete was better than today's concrete (see below).

A fundamental problem is that concrete is a man-made material, with properties critically dependent on the care taken in its preparation. Thus, the compressive strength of commercial concrete can vary from about 1,000 psi to 20,000 psi, depending primarily on the cement to water ratio used. A low cement to water ratio makes for good concrete, but also for a very stiff mix that is difficult and expensive to work with. The Romans used slave labor to ram stiff concrete into place-today's contractors like to pump a sloppy concrete through pipes.

If concrete is considered as construction material at the WIPP site, data on sulfate and chloride content of the soil are needed as well as an estimate of where the ground water table might be in the future. Our impression is that the ground water level, even if precipitation were to double, would be well below any foundation, but this must be checked with a geology expert. (If a concrete foundation should reach the ground water level, its survival for 10,0000 years would be very questionable because contact with water accelerates the erosion of concrete.)

If concrete is to be used, its preparation and testing should follow the recommendations for the preparation of concrete used in critical applications.

These applications are

- Construction of containment vessels at nuclear plants

- Construction of large offshore structures 
The concretes used in the latter applications are specifically designed to function in a wet, chloride-containing environment. Thus, these concretes should work well at the WIPP site even if salt (left over from the mining operation or blowing about as dust) or brine would generate a chloride-containing environment.

The rules below are excerpts from an article by Gerwick presented at the 1973 American Concrete Institute Conference on the Durability of Concrete [Ref. 4-3]. These rules are presented here as an example for the details that will have to be specified if concrete is used at the WIPP site. Some features, such as a water ratio below .45, a high cement content, cement with a low $\mathrm{C}_{3} \mathrm{~A}$ and alkali content, and chloride-free water for mixing and curing are common recommendations for all high quality concrete.

The following are Gerwick's recommendations for durable offshore construction [Ref. 4-3]:

- Cement -- Portland Cement, ASTM type II or equivalent. Moderate $\mathrm{C}_{3} \mathrm{~A}$ (5-6\%). Low Alkali $\left(0.65 \% \mathrm{~K}_{2} \mathrm{O}+\mathrm{Na}_{2} \mathrm{O} \max \right)$

- Cement factor -- A minimum of 7 sacks per cubic yard and preferentially 8 sacks.

- Aggregates -- To meet ASTM C33. Sound under sodium sulfate test. Satisfactory past seawater durability and freeze-thaw durability.

- Free from chlorides -- No more than $0.02 \%$ of chloride by weight.

- Non alkali reactive.

- Testing of both fine and coarse aggregate.

- Water used -- Chloride content less than $500 \mathrm{ppm}$. Sulfate content less than $1000 \mathrm{ppm}$. Water-cement ratio less than 0.45 and preferentially 0.40 . (The importance of working at a ratio below 0.45 to reduce chemical attack is also stressed by Biczók [Ref. 4-2]).

- Admixtures -- Water reducing admixtures are desirable. Air-entrainment 6\%.

- Mixing and consolidation -- Concrete must be properly mixed and thoroughly consolidated to eliminate all honeycomb, rock pockets, and "bug holes."

- Forms -- Forms should be tight, especially at corners to prevent mortar leakage. Corners should be rounded wherever possible.

- Cover -- $21 / 2$ inches of concrete over prestressing tendons and main reinforcements.

- Surface finish -- smooth. 
- Curing -- water curing should use water of same quality as mixing water. If steam curing is used, ensure that chloride content of mix is below limits set.

These are sophisticated specifications likely to baffle a local contractor. If concrete is used in the marker construction, a contractor familiar with large offshore concrete constructions or fabrication of nuclear containment vessels is therefore more likely to produce long-lasting concrete.

\subsubsection{Below-Ground, Closed Rooms}

The team recommends the use of sealed or nearly sealed (total openings below 1 square foot) rooms, buried into earth berms, man-made mounts or underground. These sealed rooms would contain Level IV information engraved on a double set of granite panels.

Below-ground structures are sheltered from temperature oscillation but may react with the soil. They are likely to last for 10,000 years if high quality concrete is used in their construction (see Section 4.4.9.9). If the soil at the site contains sulfate and chlorides, it is recommended that any concrete structure be isolated from the ground by protective layers (e.g., sand, clay).

The four sealed (or semi-sealed) units recommended by the team should be buried using sand as an intermediate layer to separate the concrete from caliche. The thickness of their caliche covering should vary such that natural erosion sequentially reveals the top of a chamber every 2,500 years. The proper design of the caliche thickness requires data on the erosion of caliche measured at the site over the next 30 to 40 years. Very small portholes (either sealed with sapphire windows, or consisting of small openings) could permit inspection of the chamber and reading of the inscriptions but must be designed to prevent physical access to the chamber.

\subsubsection{Small-Scale, Near-Surface Markers}

Small markers are proposed to be buried in the sand layer present at the site or into the caliche layer, if the sand layer is thin. The depth should be greater than the maximum depth that can be reached by plowing.

During the construction of the earth berms, scattered markers should be buried throughout, such that any effort to level those berms exposes these markers.

These scattered markers should be made from a variety of materials, such as

- Fired ceramics,

- Lanthium-borate glass,

- Plastic,

- Titanium, and 
Appendix F: Team A Report

- Magnetic markers

to ensure that even if one material fails, another subset of markers survives. If this strategy is followed, it is virtually certain that scattered markers will survive for 10,000 years at the WIPP site.

The attractive feature of "classical" ceramics, such as silica and alumina, is that they are already oxides and therefore guaranteed resistant against further oxidation. This sets these materials apart from metals (except the noble metals) and modern ceramics such as carbides, nitrides, and borates. A ceramic that occurs in nature as a mineral (e.g., silicon dioxide, quartz and aluminum oxide, sapphire) is more likely to survive for long periods than one that does not (e.g., silicon nitride).

The durability of fired ceramics improves with the firing temperature. Sumerian cuneiforms prove that fired clay is durable, but modern ceramics should also be considered. Technical porcelain (as used in high voltage insulators) has an excellent service record under demanding conditions and therefore should be considered for scattered markers. Other candidates are beryllium oxide and aluminum oxide. Single crystal aluminum oxide (sapphire) is extremely tough and corrosion resistant (which explains its survival as a gem stone in the ground). Suitable sapphire disks are made in large numbers commercially by Corning and Union Carbide (sapphire wafers are used in the electronic industry to make radiation hard circuits).

Of the modern ceramics, silicon nitride and zirconium stabilized yttrium oxide (a material with a relative high fracture toughness) would be candidates for scattered markers.

Glass is an amorphous oxide, and, in a dry environment, is likely to survive 10,000 years. Low melting soda-lime glass from Egyptian times has survived (with erosion) to date. In a wet environment, soda-lime glass is fairly resistant to acids and moderately resistant against alkali. According to Dr. Leroy Morse of Corning Glass Laboratories, Corning glass has an experimental glass, lanthiumborate, developed in the program to vitrify nuclear waste that is "much" more resistant to corrosion than regular glass or even .Corning laboratory glassware (Pyrex). He estimated the cost for lanthiumborate glass to be a "few" dollars per pound. Markers made of this glass could contain colored cores shaped as icons.

Plastics, i.e., organic polymeric materials, are not usually associated with durability. However, some "plastic" materials such as heavy tar have survived in the ground for millenia (which explains why tar pitch has an excellent service record as a protective covering in the pipeline industry). Plastic is cheap, and plastic markers can be fabricated in great numbers and with various colors.

Unfortunately, not much literature exists on the survival of modern plastics in the ground except for studies of the problem of disposal of plastics in landfills and what plastics to use to line landfills against seepage. For this purpose polyethylene is used. It has a very good service record but, of course, the experience with buried polyethylene is too short to extrapolate with confidence to 10,000 years. However, as a saturated hydrocarbon compound, chemically similar to oil, polyethylene may well survive for 10,000 years in the ground. (This is especially likely 
for polyethylene buried in salt.) Thus, it is recommended that a subset of the markers may be made out of polyethylene. Polyethylene and any other plastic is not recommended for aboveground duty as it will deteriorate in sunlight.

Metals are materials that are easily reusable (e.g., by melting) and therefore unlikely to survive at the site except, perhaps, as subsurface markers. If a metal were to be used, the clear choice is titanium [Refs. 4-12, 4-13, 4-14]. Only a small subset of markers should be made out of this metal to make mining an uneconomical prospect.

Titanium owes its high corrosion resistance to its pronounced tendency to oxidize. Therefore titanium is always covered with a layer of titanium oxide. It is this self-healing ceramic coating that accounts for the high corrosion resistivity.

Thus, unalloyed titanium is highly resistant against the corrosion normally associated with many natural environments, including seawater, body fluids, and fruit and vegetable juices. Wet chlorine, molten sulfur, many organic compounds, and most oxidizing acids have essentially no effect on this metal. Titanium also resists hydrogen sulfide and carbon dioxide gases at temperatures up to $500^{\circ} \mathrm{F}$ [Ref. 4-14].

Magnetic markers are proposed to be buried in berms only. The markers should be buried centrally, at the base of the berms (i.e., 50 feet below the top surface) to make retrieval difficult. The magnets should be sized such that their magnetic field at the surface of the berm exceeds the earth's magnetic field by a factor of 10 . Consideration should be given to the direction of the magnetic field that is induced. These fields could point toward a buried Level IV storage site, or simply be oriented such that they locally reverse the direction of the earth's magnetic field.

Magnetic markers are likely to survive for 10,000 years if protected against corrosion. Therefore, the permanent magnetic material (e.g., Alnico) used in the construction should be encased with polyethylene and a hermetically sealed titanium housing to protect the markers from corrosion.

\subsubsection{Deeply Buried Markers}

Such markers are proposed to be located in the shafts and the waste panels.

The repository itself may be detectable by several methods commonly used today in geological explorations. Induced polarization techniques can detect the metals deposited (containers, tubing, etc.). Magneto and telluric field techniques as well as electrical resistivity can detect the presence of a conducting medium (especially if a brine slurry should form). Gravimetric (static), magnetic, seismic, self-potential, and radioactivity methods appear less promising. Even when detected, however, the repository's detailed shape is unlikely to be discernible, only its rough location and size. We have therefore not proposed shaping the waste panels into any special 
Appendix F: Team A Report

form. ${ }^{20}$ The access shafts are the most likely paths for migration of radionuclides by natural processes. For this reason, the access shafts should be specifically marked.

The shafts should be marked by filling the top 50 feet with alternating layers of sand (10 feet) and gravel. The sand layers should contain fired clay, glass, and titanium markers containing pictorial and verbal information showing that this is a backfilled access shaft.

The message on these markers could deviate from the general message in pointing out that these shafts are the most likely site for the up-migration, if any, of radioactive material. Because the sealing techniques and migration rates have not yet been established, the specific wording of this message remain for future panels to decide.

In addition to markers, the sand layers might contain "monitoring" materials to offer future generations the possibility of tracing the migration of alpha-emitting materials. An example is the plastic found in radon monitors (decaying radon introduces nuclear tracks that can be selectively etched and thus counted). The design of these monitors requires further study. Slant drilling (and even drilling from below) has been considered by the Futures panel. Horizontal drilling (within the reach of today's technology) can only be cautioned against by locating markers at the depth level of the waste panels.

Slant drilling could be warned against from the surface, but to warn against shallow angle drilling would require marking a very large surface area. (To take an extreme example, surface warning against drillers using a 15-degree angle from the horizontal would have to cover almost 2 miles beyond the footprint of the waste panels.) This could be done, but would dramatically increase the marked area of the site.

The panel has therefore considered markers at the depth level of the waste panels.

Small scattered markers of the type recommended to salt the surface are likely to be ineffective in warning against unintentional drilling into the waste panels, as they most likely would be not noticed by the drilling crew.

To ensure attention to the presence of markers, a marker system must be used that forces the driller to inspect the properties of the layers they are drilling into.

Any material that will survive for 10,000 years in salt and that exhibits very different drilling properties from salt will do (whatever the drilling technology will be in 10,000 years, it will be maximized to make progress in salt). A material should be selected that is physically hard and has a high heat of evaporation (to take care of the laser drilling schemes considered by the Futures panel).

20 We thank Bob Guzowski for this information. 
Thus, large chunks of rock, or slabs made from concrete resistant to seawater appear suitable.

Disruption of the drilling process will likely lead to an inspection of the material causing the disruption. Therefore, once we have the attention of the driller, we need to have additional markers that can easily be retrieved (or are retrieved automatically by the drilling fluid or the bit). This consideration restricts the size of these markers to the size of fragments generated by the drilling process itself.

Thus, these large blocks should be interspersed with small markers of clay, plastic (which in this dark and constant temperature environment may well survive), glass and (if affordable) titanium clad magnets containing Level II warnings. The magnetic marker should be designed to optimize adherence to drill bits (a test is recommended as well as the exploration of other schemes that would adhere markers to drill bits such as the use of cold welding).

If the marker surface is too small to contain the entire Level II message, the message should be spread over two or three markers, together with an obvious symbol on how to paste the markers together.

The location of these markers is dictated by two considerations:

- Slant, and even horizontal, drilling was considered by the Futures panel.

- An early warning is desirable.

To meet the first requirement, the outer end-walls of the waste panels should be backfilled with the above mixture, and they could not be used to store remotely handled waste. Also unusable would be those sections of the sidewalls that can be hit by horizontal drilling in directions other than those within say 15 degrees of the orientation of the long axis of the waste panel.

The remainder of the sidewalls could be used because rooms shield each other.

The second requirement would be best met by excavating a (thin) layer of salt above the waste panels, to be backfilled with the above mixture. However, excavation of a layer for the deposition of markers violates the principle of disturbing the layering (and hence the long-term stability) of the site as little as possible. The tradeoff is unclear and deserves future study.

If a separate layer cannot be excavated, the top of the waste rooms should be backfilled with the above mixture. 


\subsection{Graphic designs for markers}

\subsubsection{Design Criteria}

In keeping with the considerations presented in Section 3.2, we recommend the following design criteria for the use of graphics.

- They must have emotional impact. Given that people coming on the site may be unable to read any verbal message, they must be impressed by the site itself as a symbol evoking awe and apprehension. Visual symbols and the icons must be evocative in the same way, reinforcing the impression given by the arrangement of the site's structures. Evocative symbols and icons must be located so that they are among the first things people encounter at the site.

- Whatever graphics are used, their nature and location should be such as to make them an integral part of the site as a whole, every part and feature of which must reinforce every other part with cumulative emotional and informative impact.

- Graphics must be unambiguous and universally meaningful cross-culturally. In this regard, icons most suitable for evoking wariness and apprehension are representations of human faces exhibiting the expressions people universally associate with such states as horror, revulsion, fear, pain, and anguish.

Human faces or other graphics may be used by themselves, but are better used in conjunction with language. For instance, if human faces are used to frame the shortest word messages, such as "DANGER, poisonous radioactive waste buried here," they will indicate that the message is a warning and invite its decipherment as a precaution to any intrusion on the site. The representations (Figures 4.5-1 and 4.5-2) on the following page are suggested as possible examples for appropriate artistic adoption [Ref. 4-15, 4-16].

- Symbols and words should be used together in ways that allow each to aid in the interpretation of the other. Their ability to communicate will be enhanced if they are mutually reinforcing. Thus the word "danger" and iconic signs that suggest danger will be more likely to communicate if exhibited together than if exhibited separately, particularly in messages of Level II. Similarly, telling people not to dig is for some an invitation to dig, arousing curiosity as to why they are being told not to. A warning against doing something must be accompanied by symbols that evoke a sense of danger or fear and also by an explanation for the warning. 


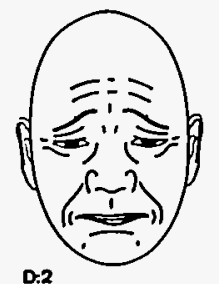

Mourniul, almost in tears

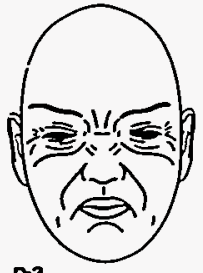

Physically hurt, tormented

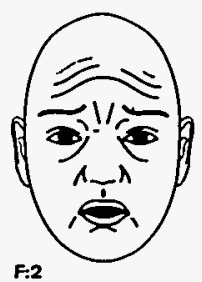

Frightened

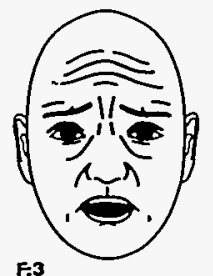

Panicstricken, anguished

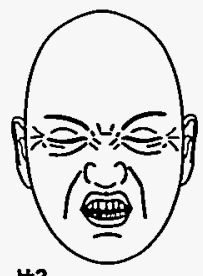

Nauseated

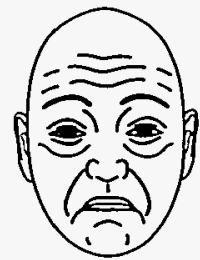

+43

Bitter, woeful

Figure 4.5-1. Possible prototypes for facial icons, example 1. See text for details. Reprinted with permission from: Eibl-Eibesfeldt, Iranäus. Human Ethology. (New York: Aldine de Gruyter) Copyright $\odot 1989$ by Iranäus EiblEibesfeldt.

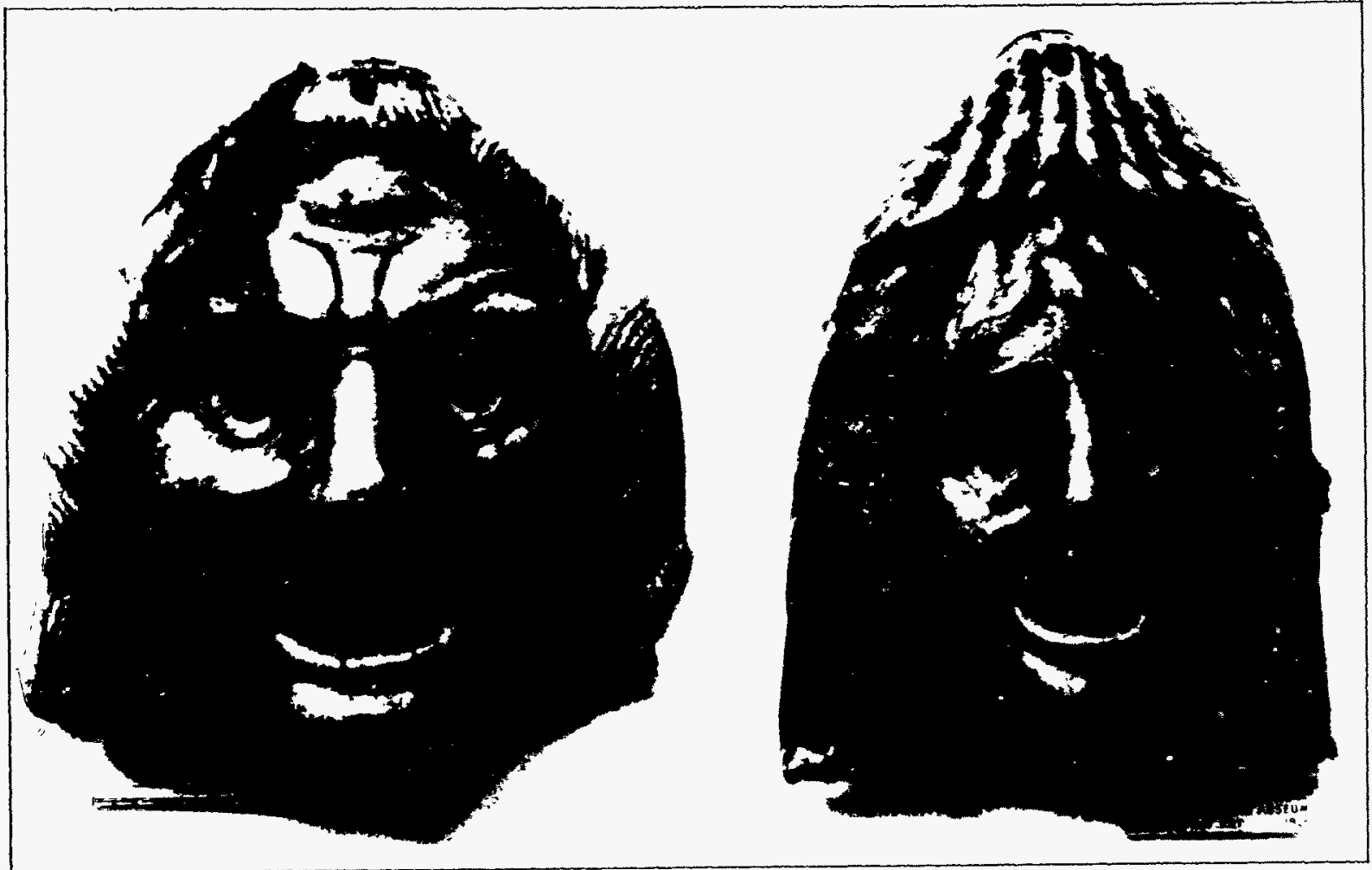

Figure 4.5-2. Possible prototypes for facial icons, example 2. See text for details. The Metropolitan Museum of Art, Carnarvon Collection, Gift of Edward S. Harkness, 1926. (26.7.1020, .1021). All rights reserved, The Metropolitan Museum of Art. 
- Messages of greater length than those of Level II should be introduced in each case by the short "DANGER" message framed with the facial icons-the Level II message first encountered on entering the site-in order to indicate that the longer messages are fuller explanations of the danger warning in Level II.

- Conventional symbols, if used, should be the ones, such as mathematical and scientific symbols, that have wide international recognition and use, regardless of other differences in language and culture. There is more likelihood that such symbols will persist in use or, at least, be understandable to historians in the distant future.

- Symbols and words must be clearly legible. Human figures and faces are clearer in base relief than when incised. Words are clearer when incised. Their size and shape must be adapted to the needs of the viewer under the conditions in which they are to be seen and read. Engravings and text must have an incised depth sufficient to survive 10,000 years of erosion. Level III messages should be further protected from erosion by the use of shielding walls, and Level IV messages will be best protected by being in enclosed rooms.

- Visual representations of the site's stratigraphy and the location of the buried waste therein may usefully accompany the fuller explanatory statements to be inscribed in the Level IV message chambers.

- Conventional symbols relating to scientific matters are best confined to the full explanatory statement (Level IV) and used in association with the appropriate words in the text. For example, the trefoil design may continue through time as an international symbol of radioactivity and could be appropriately used in conjunction with mention of radioactivity in the explanatory inscriptions, but only there in textual context. Similarly, the periodic table of elements and the conventional signs representing them are likely to be known at least to historians of science, if not to scientists, thousands of years hence. Their use in conjunction with the full explanatory statement is therefore appropriate.

The sections to follow give details of the various graphics that we recommend. We have not included any pictographs, but have no objections to them if they can be kept simple in design and yet reasonably unambiguous.

\subsubsection{International Symbol for "Buried Radioactive Waste"}

A difficult question is whether or not to include the familiar radiation hazard trefoil as a part of our design. It is indeed an internationally recognized symbol with a 40-year history, but its long-term intelligibility when applied to all cultures over a period of 10,000 years is dubious at best. Furthermore, one of its standard uses means "do not go into this space unless properly 
protected," whereas we are not trying to keep people away from the surface above the WIPP repository. So even if the symbol were understood in the future, once no radioactivity was measured on the surface, we might lose our credibility in the eyes of future investigators. We have compromised by not only making the trefoil a vital part of our design (such as by arranging monoliths or berms in the form of the trefoil), but also by not ignoring it altogether. We propose to insert the trefoil in all texts of all levels after each occurrence of the word "radioactive" and also for the appropriate elements in the periodic table (see Section 4.5.6). In this way, we define its meaning for those who can understand the language or the periodic table, and we give some warning to those who know the symbol but not the language. In order to avoid the ambiguity mentioned above, we propose always to incorporate the trefoil with a downward arrow, meaning "the radiation is not here, it is below" (Figure 4.5-3).

Perhaps, a better overall symbol to incorporate into the marker system in a major way would be a (new) international symbol specifically for "long-term radioactive waste buried here." This symbol would be used at all disposal sites as well as appear on all reports dealing with radioactive waste. In this way, its meaning would become well-established and its recognition at a site would immediately convey our basic message. The design of such a symbol would again

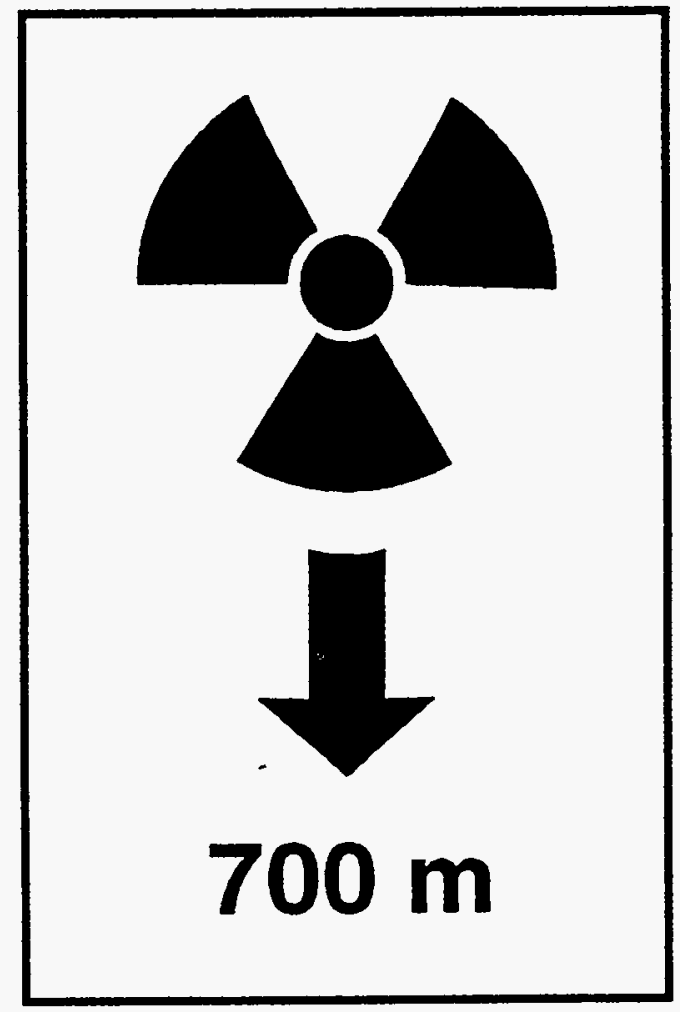

Figure 4.5-3. Proposed sign for buried radioactive waste. 
be the task of an international commission; the symbol would then become part of the international standard for marking disposal sites.

The 1984 Human Interference Task Force recommended creation of a specific symbol for "biohazardous waste buried here"; because such a symbol encompasses a much broader class of wastes, it would indeed be ubiquitous. On the other hand, the special and very long-term dangers of radionuclides are distinctive enough that we recommend a symbol confined to radioactive waste burial.

\subsubsection{Faces}

As discussed earlier, we strongly recommend the inclusion of drawings of faces expressing emotion as a major part of the marking system. These are most appropriate for Level II, which is the simplest explicit message and will be engraved on just about every available surface all over the site. Fig. 4.5-4 shows one realization of the Level II message as flanked by two faces. The left face (and associated hands) conveys abject horror and terror (not unlike Edvard Munch's famous painting 'The Scream'); the right face conveys disgust, as for something nauseating or poisonous. In our example given here in Fig. 4.5-4, the second face is a bit more detailed than desirable, and the first (without the services of an artist) perhaps not detailed enough.

\subsubsection{Maps}

There are two classes of maps that we recommend: (1) sites around the world and (2) the WIPP site. In Section 4.3, Fig. 4.3-16, we show the option of a very-large-scale map that would be a major element of the overall site design. Whether or not this is adopted, we recommend that the Level IV room should include a world map (Fig. 4.5-5) showing all radioactive waste disposal sites, each indicated by the (new) international symbol discussed earlier. The WIPP site should be located at the center of the map and therefore serve as a point of reference for locating other sites whose marker systems may have failed for cultural or physical reasons. The map itself might be about 1-m across on the wall, and thus an engraving accuracy of 1-mm would only locate each site to an accuracy of about $40 \mathrm{~km}$. (By the way, continental drift fortunately will amount to only a few hundred meters over 10,000 years.) In order to improve this locational accuracy by an order of magnitude, we suggest that an adjacent diagram indicate the latitudes and longitudes of all sites relative to that of the WIPP site. The WIPP site is the obvious reference frame for $(0,0)$, because the future reader will know it, and not need any historical knowledge about Greenwich, England, 10,000 years before. This diagram (Fig. 4.5-6) consists of a partial circle that indicates relative latitude within a full circle indicating relative longitude. The numbers associated with each dot correspond to a site that is similarly numbered 

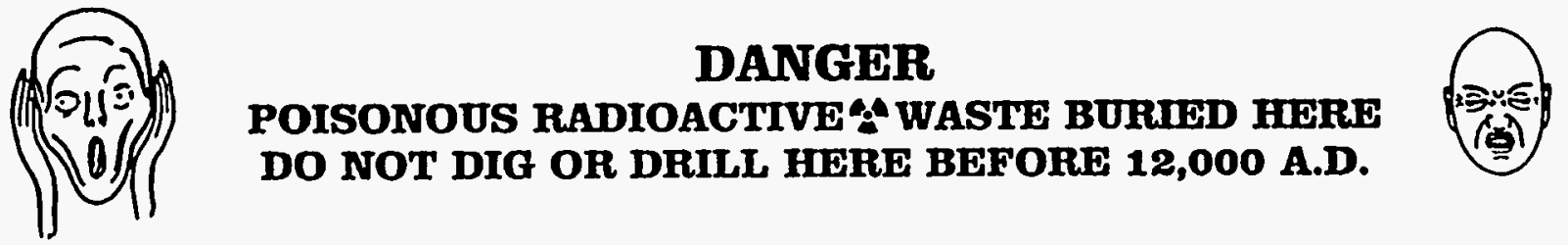

Figure 4.5-4. Proposed Level II message. The face to the right is reprinted with permission from Eibl-Eibesfeldt, Iranäus. Human Ethology. (New York: Aldine de Gruyter) Copyright ${ }^{\odot} 1989$ by Iranäus Eibl-Eibesfeldt. 


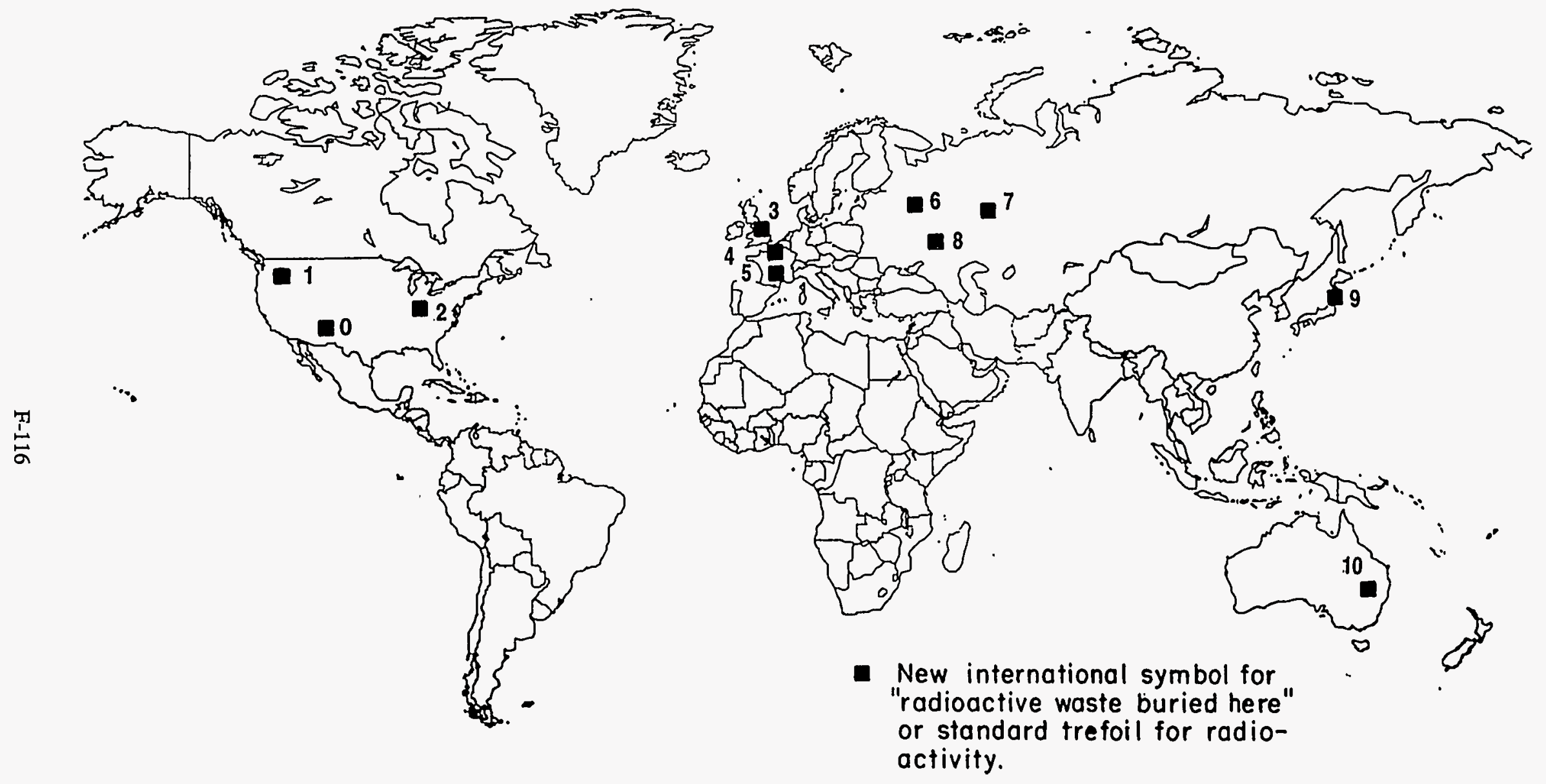

Figure 4.5-5. Example of a map in the Level IV chamber indicating the locations of nuclear waste sites around the world. 


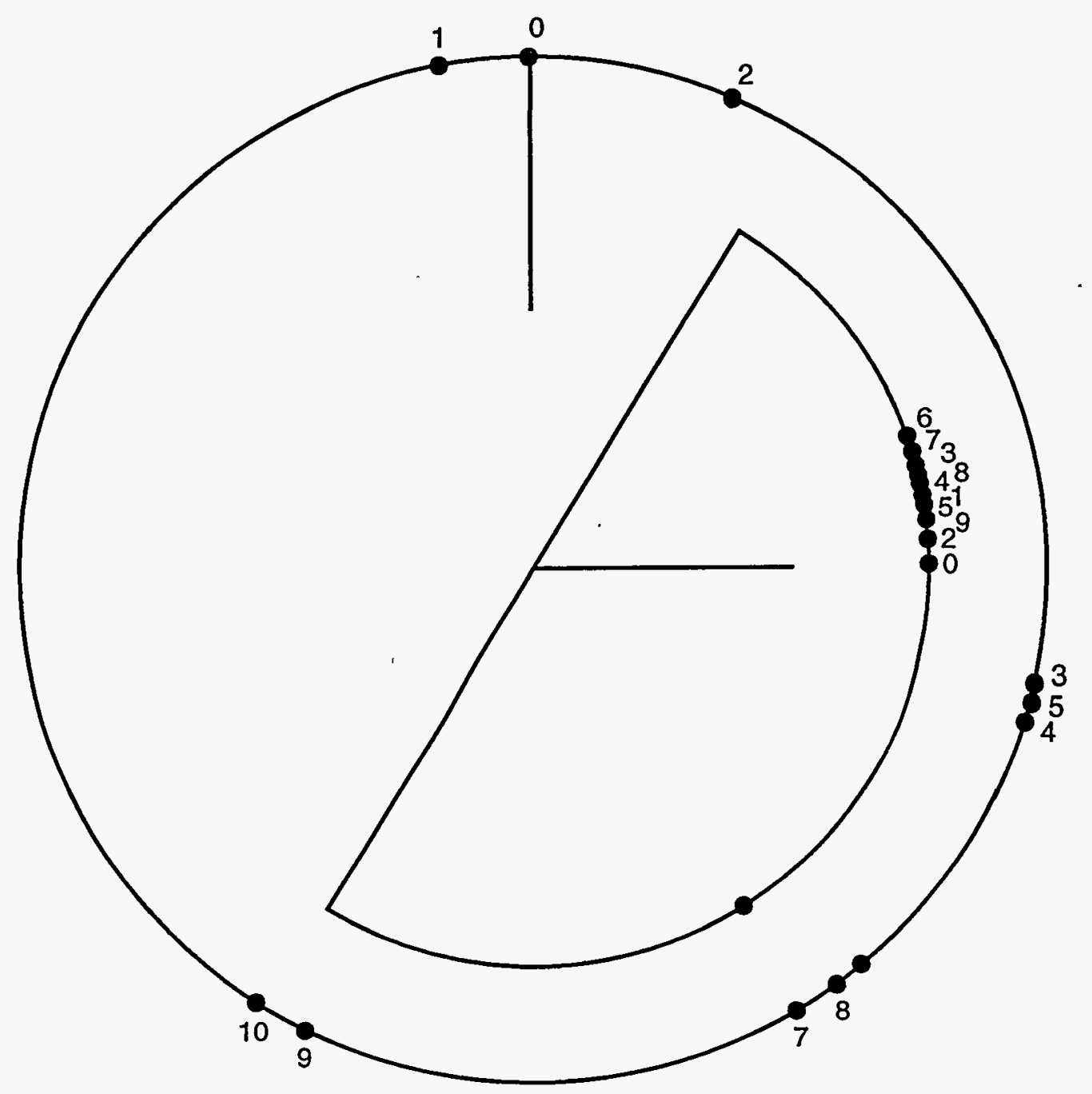

Figure 4.5-6. Diagram for Level IV message indicating much more accurate locations for the sites shown in Fig. 4.5-5. If the circle has a diameter of about $3 \mathrm{~m}$ and the dots on its circumference are drawn with a positional accuracy of $1 \mathrm{~mm}$, sites can be located to about $4 \mathrm{~km}$ accuracy. The text explains how the outer circle indicates the longitudes and the inner semicircle the latitudes of all sites. 
on the map. Ambiguity between longitude and latitude is averted because the latter has a limited range in its values (for WIPP at lat. 32 degree, the range is -122 degree to +58 degree), whereas longitude extends over a full 360 degrees. The circles have a diameter of 3-m, meaning that a 1-mm engraving accuracy allows a 4-km locational accuracy (about 2-arcmin of angle). A 3-m circle takes up a lot of wall space, but in fact most of its interior will be empty and can be used for text. The map and circles should also be part of an international standard, thus interlocking all site locations with each other.

The second type of map is of the WIPP site itself. We recommend that a perspective view accompany each Level III message. This view (Fig. 4.5-7) shows "to scale" surface features of the marking system, the reader's present location on the surface, shaft locations, and the layout of the waste storage panels. Combined with the faces on the Level II message, this Level III graphic conveys--even to someone who does not understand the language--the idea of horrific stuff buried at a specific depth.

Level IV will contain a more detailed version of Fig. 4.5-7 as well as plan views of the marker system and the repository. It will also have a side view showing the geological strata and the location of the repository. These diagrams are not problematic or novel and so are not shown here. The Level IV room should also contain a three-dimensional carved block of granite that indicates both the site's topography and the location and shape of the repository (same scale in all three dimensions). This model will cover the situation in which conventions of perspective on a two-dimensional graphic are not understood.

\subsubsection{Star Map Showing Precession}

The astronomical phenomenon of precession allows us to indicate the date of the site, as well as time intervals. The projection of the earth's north pole, now fortuitously pointed very nearly toward the star Polaris, actually moves and describes a circle on the sky of radius 23.5 degrees and period 26,000 years. Any culture (even low-tech) that watches the stars will know where the pole for their own epoch lies, although it takes more astuteness (in the case of Western culture, Hipparchus) to notice, say over a period of a few centuries, that the pole's location has changed. The shapes and relative locations of the constellations, however, do not significantly change (for our purposes) on a 5,000- or 10,000-year scale. Thus, a simple diagram of the northern sky showing three major constellations and (prominently) the position of the pole, nicely indicates the epoch $\mathrm{AD} 2000$ (easily to 100 years accuracy). In addition, a time interval (such as the half-lives of the main constituents of the waste) can be indicated by such a diagram having a trace over a portion of the full circle, e.g., one fourth the way around indicates 6,500 years. 


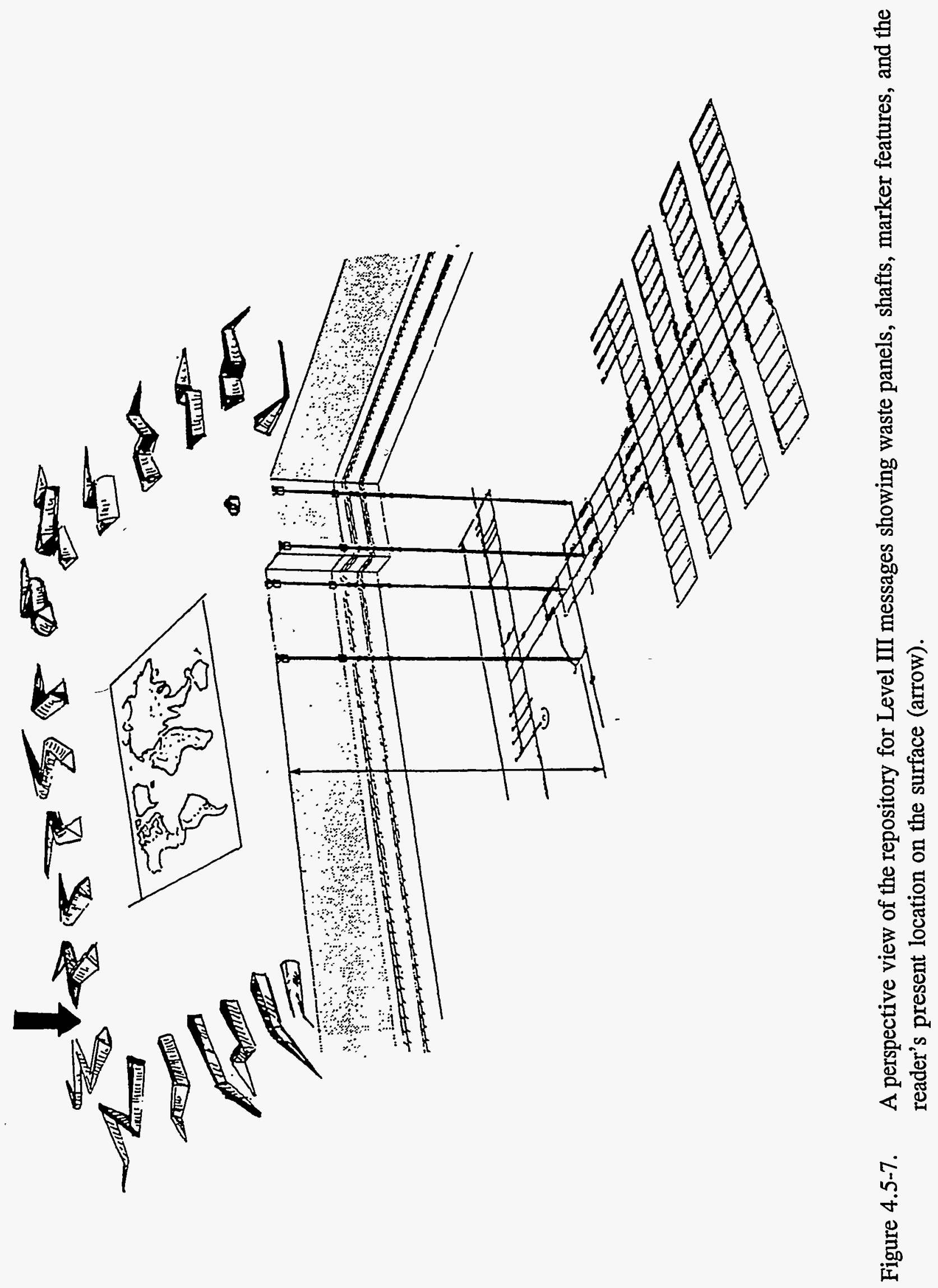


We propose to use a precession diagram with the Level III message (Fig. 4.5-8). To those not able to understand any languages, this diagram will indicate both the epoch of burial and the period of danger. The diagram shows a progression from a disgusted to a neutral face to a more content face as the epoch changes from AD 2000 to $\mathrm{AD} \mathrm{12,000} \mathrm{to} \mathrm{the} \mathrm{millennium} \mathrm{beyond.} \mathrm{Also}$ along this arc of the full precession circle is a sequence of the (new) international symbol (for buried radioactive waste) steadily decreasing in size, symbolizing less danger as time passes. The Level III message will thus be accompanied by two diagrams that, independent of the language, characterize the nature of the waste and its location in space and time. Level IV will also utilize the precession diagram, but to indicate the half-lives of radionuclides (next section).

\subsubsection{Periodic Table of Elements}

The Level IV message will contain a diagram showing the periodic table in its usual form (Fig. 4.5-9). Where possible, the box for each element will contain a small plug of the actual element itself. Those elements that are naturally radioactive will have a radiation trefoil in their boxes. Those elements that have radionuclides in significant quantity in the WIPP repository will also have the (new) international symbol for "radioactive waste buried here," along with arrows or a connecting line linking each of them to the repository portion of the diagram showing the WIPP perspective view (Fig. 4.5-7). Furthermore, each of these WIPP radionuclides will have its half-life indicated by a precession diagram with the appropriate fraction of the 26,000 -year circumference circle marked out.

\subsection{Marker messages, Levels II, III, and IV}

\subsubsection{Message Levels, Languages, and Markers}

The Level II and Level III messages should be short enough so that they can be inscribed in the six languages of the United Nations plus a possible local language such as Navajo. It does not seem feasible to inscribe the longer Level III message on each marker in each language. Each marker should have its Level III message in the (20th century) local language (i.e., English at the WIPP site) and the others, with a rotation system ensuring that all the non-local languages be equally represented. As far as the Level IV message is concerned, practicality might dictate that it be given only in one language. If so, it should be in English. If there is room for it to be given twice at the WIPP site, the second language should be Spanish. 
0

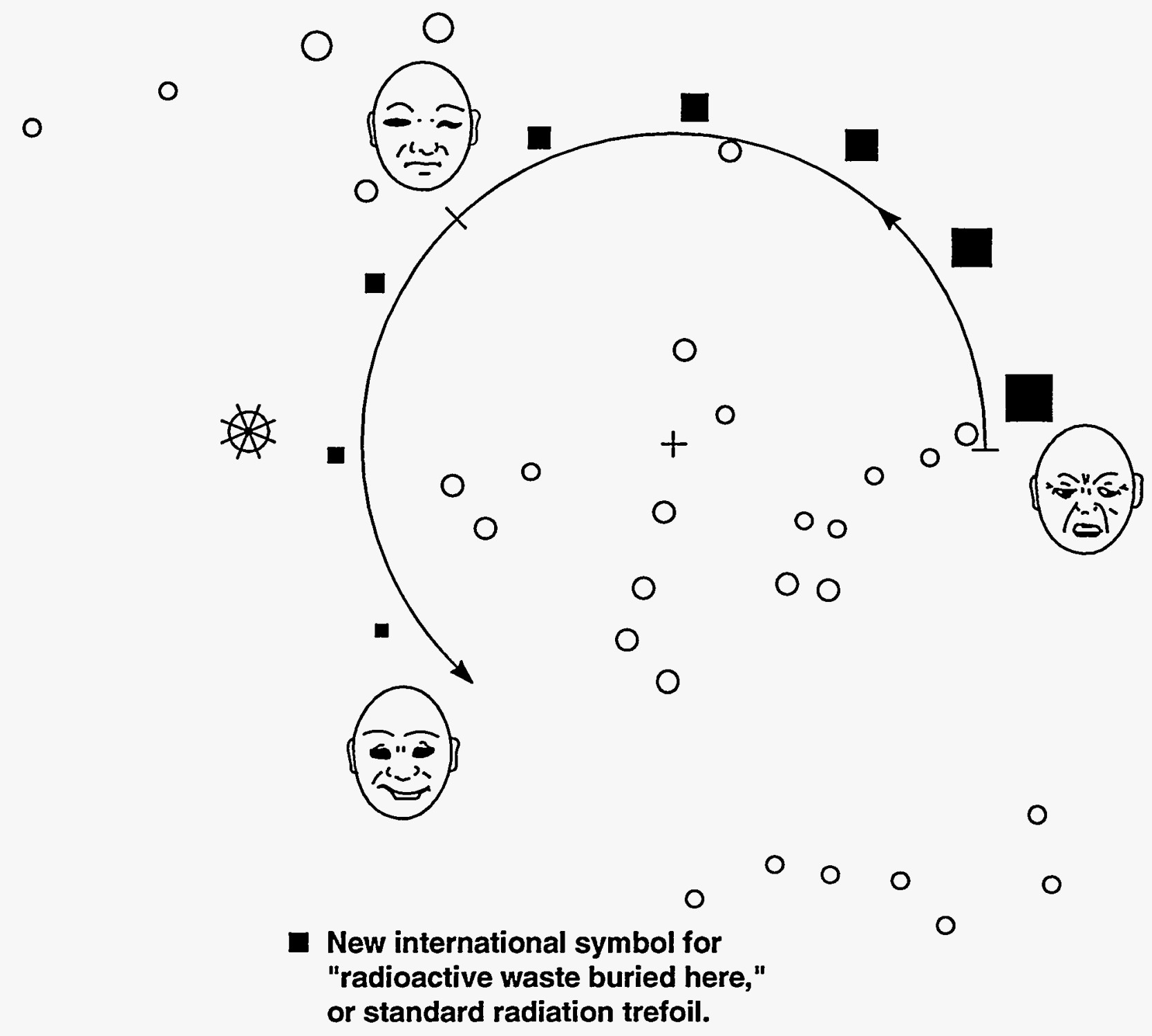

Figure 4.5-8. Level III diagram that allows the date of burial and the time that has passed since burial to be determined if precision of the pole is a known phenomenon. As the position of the pole moves from the right (near the star Polaris and the unpleasant face), the size of the symbol for "radioactive waste buried here" (here shown as a filled square) steadily shrinks. The face becomes a neutral one after 10,000 years and a more content one later. Faces based on figures from Eibl-Eibesfeldt, Iranäus. Human Ethology. (New York: Aldine de Gruyter) Copyright ${ }^{\odot} 1989$ by Iranäus Eibl-Eibesfeldt. 


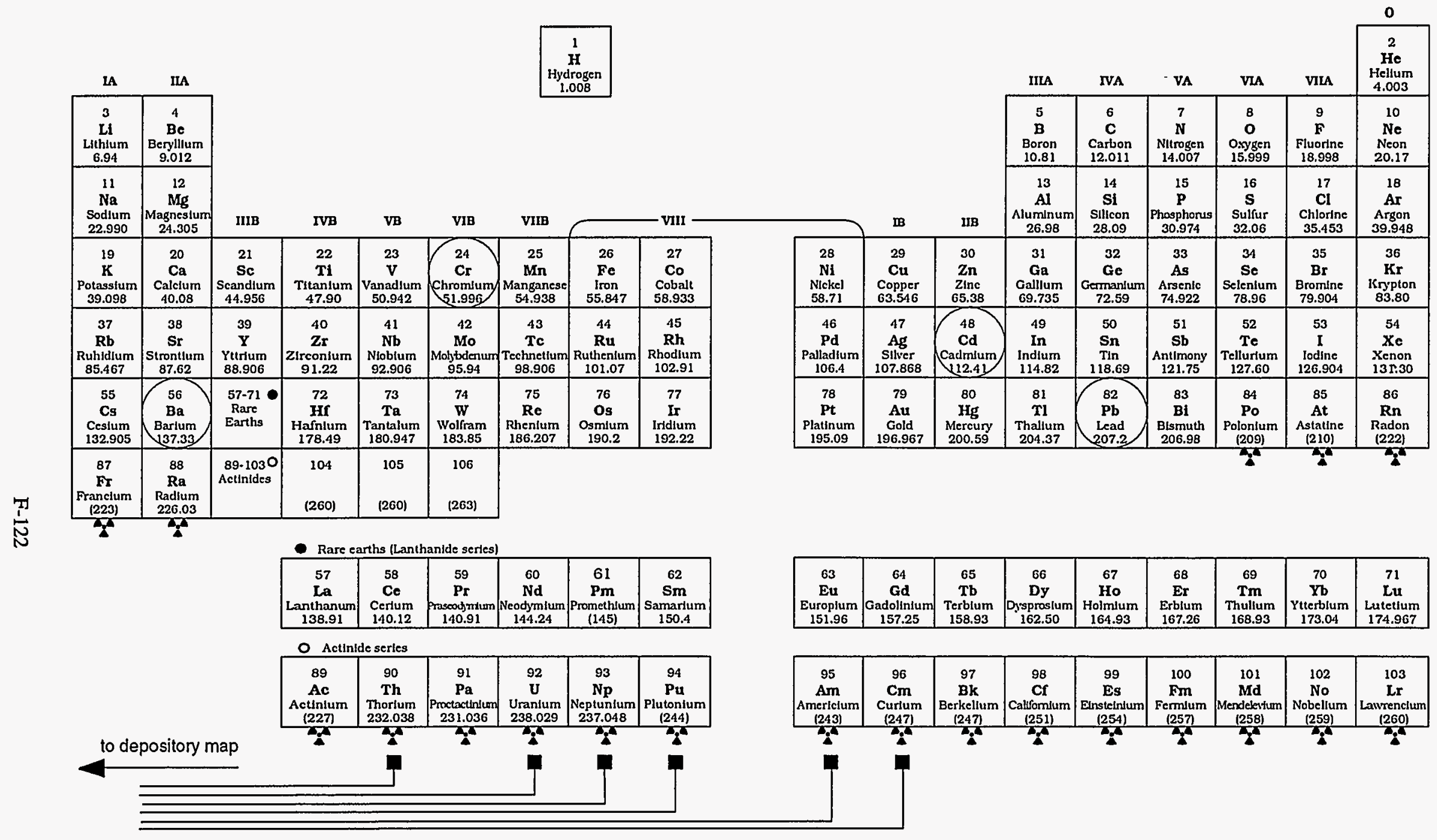

Figure 4.5-9. Level IV diagram of the periodic table of elements. Radioactive elements are indicated with the radiation trefoil and those with major amounts in the repository are further labeled with the symbol (here shown as a filled square) for "radioactive waste buried here," as well as a connecting line to a map of the repository. Non-radioactive toxic elements present in the repository are circled. 


\subsubsection{The Messages Themselves}

\subsubsection{Marker Message, Level II}

We suggest the following Level II message:

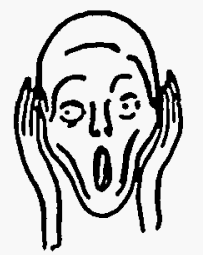

\section{DANGER POISONOUS RADIOACTIVE $Q$ WASTE BURTED HERE DO NOT DIG OR DRILL HERE BEFORE 12,000 A.D.}

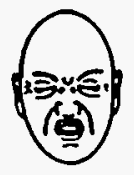

Face on the right reprinted with permission from: Eibl-Eibesfeldt, Iranäus. Human Ethology. (New York: Aldine de Gruyter) Copyright ${ }^{\circ} 1989$ by Iranäus Eibl-Eibesfeldt.

\subsubsection{Marker Message, Level III}

We suggest the following Level III message:

These structures mark an area used to bury radioactive wastes. The area is...by...kilometers and the waste is buried...kilometers down. This place was chosen to put this dangerous material far. away from people. The rock and water in this area may not look, feel, or smell unusual, but may be poisoned by radioactive wastes. When radioactive matter decays, it gives off invisible energy that can destroy or damage people, animals, and plants.

Do not drill here. Do not dig here. Do not do anything with the rocks or water in the area.

Do not destroy this marker. This marking system has been designed to last 10,000 years. If the marker is difficult to read, add new markers in longer-lasting materials and copy this message in your language onto them.

For more information, go to the building further inside this marked area. The site was known as the WIPP (Waste Isolation Pilot Plant) site when it was closed in....

\subsubsection{Marker Message, Level IV (first alternative)}

We have developed two sample Level IV messages. Straight brackets, [ ], enclose comments for this report. The shorter of the two reads are follows:

This place is a burial place for radioactive wastes. We believe this place is not dangerous 


\section{IF IT IS LEFT ALONE!}

We are going to tell you what lies underground, why you should not disturb this place, and what may happen if you do. By giving you this information, we want you to protect yourselves and future generations from the dangers of this waste.

The waste is buried...kilometers down in a salt layer. Salt was chosen because there is very little water in it and cracks caused by digging the rooms for the waste reseal. There is a pocket of pressurized salt water...kilometers below the waste. There is a rock layer...kilometers below the surface that did not have drinkable water when we built the site. We studied all the things that could go wrong with the site. We found out that the worst things happen when people disturb the site. For example, drilling or digging through the site could connect the salt water below the radioactive waste with the water above the waste or with the surface. The salt water could wash through the waste and bring the poisonous and radioactive waste to the water near the surface or to the surface itself. People who drink the water will drink the poison. If the water is used for animals or crops, those too will be poisoned and the people who eat them will be poisoned. It may take many years for the sickness and death to show. Radioactivity poisons people because it can cause cancer. When radioactive matter decays, the energy it releases can damage the basic material of life in each cell of the human body. The damage can cause uncontrolled cell growth, called cancer, that can kill.

The waste is buried in 845,000 metal drums in a space of about $6,200,000$ cubic feet. The waste was generated during the manufacture of nuclear weapons, also called atomic bombs. The waste is basically laboratory and manufacturing materials that are contaminated with radionuclides having atomic numbers greater than 92, half-lives exceeding 20 years, and concentrations exceeding 100 nanocuries per gram. (A gram of radium is a curie of radioactivity. There are 1,000,000,000 nanocuries per 1 curie.) The waste includes metal objects (such as hand tools, machine tools, and motors), glass objects (such as cups and containers), plastic objects (such as bags, tubes, and gloves). Paper and rag materials, such as protective clothing worn by people when they worked with the radioactivity, will decay after burial, but the radioactivity will remain.

Pictures on the walls of this room help explain the message. A map shows the surface marking system, its relationship to the underground area used for disposal, and the depth of the waste disposal. 
There are four other rooms like this one at the site. A map shows the rock layers below the site. A periodic table of elements identifies those elements that are radioactive and those that are buried below here. When the site was closed in..., it contained:

$$
\begin{array}{ll}
\text { plutonium-239 } & =\ldots \text { curies } \\
\text { plutonium-240 } & =\ldots \text { curies } \\
\text { americium-241 } & =\ldots \text { curies } \\
\text { uranium-233 } & =\ldots \text { curies } \\
\text { thorium-229 } & =\ldots \text { curies } .
\end{array}
$$

Radioactivity declines exponentially with time. By 10,000 years after the waste was buried here, the waste will be no more hazardous than the ore from which the radioactive material was taken [see 50 FR 38071a]. There is a picture with the four brightest stars that can be seen from the site (Sirius, Canopus, Arcturus, and Vega). The position of the star-rise changes in time, and lining up the angles of the star-rise with the map will show how much time has passed since the site was closed. The site was closed in ...AD (anno domini), Gregorian calendar...AD, Byzantine calendar..., Jewish calendar..., Islamic calendar..., Chinese calendar....

The waste also contains hazardous materials, whose danger does not lessen with time. These materials include: lead, cadmium, chromium, barium, methylene chloride, and toluene. The elements also have an arrow in the box in the periodic table. The chemical form for methylene chloride and toluene are shown, also.

If you find unusual sickness in this region, or you find higher than normal levels of radioactivity in the area, inspect the area of the site. Look for: boreholes that were drilled after the site closed, but were never sealed; old mine shafts that were never sealed; and failed seals from the original repository. Reseal these areas, using your best technology, to prevent any further leakage of radioactivity or toxic materials.

Do not destroy these markers. If the message is difficult to read, rewrite the message in your language in the blank area on this wall. If the markers are worn or missing, add new markers in longerlasting materials in languages that you speak. This site, built in ...by the United States of America government, represents a first attempt to responsibly dispose of wastes for an extended period of time. Other sites exist that contain radioactive wastes, and they are 
marked in a similar manner. We have shown these sites on a map in this room. Do not disturb any of these sites.

\subsubsection{Marker message, Level IV (second alternative)}

Our second sample Level IV message is longer and more informative than is absolutely necessary for the basic tasks of the marker system; it consists of about 2500 words and 7 illustrations. If this message is deemed too long either for practical or policy reasons, then suggested cuts (mostly of historical information) have been indicated by printing these sections in smaller type. Straight brackets, [], enclose comments for this report. As it stands now, many of the stated "facts" in this version are tentative and need checking.

This version of a Level IV message is written as if the current date is AD 2020 and the WIPP is being sealed. It is written from the point of view of the builders and operators of WIPP, who are speaking to any future persons who might come upon the Level IV chamber, giving them information they need or would like to know. These persons would primarily include engineers and scientists who are trying to understand the physical waste storage area, as well as historians and archaeologists who wish to study 20th-21st century culture. Explained in detail are the rationale for the repository and marker system, as well as all diagrams appearing in this message and in the Level II and Level III messages.

This place is a repository where radioactive waste has been buried. It was designed to isolate dangerous radionuclides from humans and other life forms for a period of at least 10,000 years. The repository is at a depth of 650 meters below this room [a line of one meter length $(1 \mathrm{~m})$ is shown under this text]. DO NOT DRILL OR DIG AT THIS SITE, OR DO ANYTHING ELSE THAT MIGHT DISTURB THE WATER OR ROCKS IN THIS AREA. If you do, there is danger that the poisonous radioactivity may come to the surface in the ground water. If this water is used directly by humans or for growing food or feeding animals that produce food, humans could suffer from the disease cancer. Cancer is the uncontrolled growth of cells in the human body and can result from the damage to cells caused by the energy from decaying radioactive materials. It sometimes takes many years for the sickness and death due to cancer to become evident. If you suspect that radioactivity may have reached the surface, check this site for (1) failed seals in the shafts of the original repository, Diagrams 1 and 2 [shown in Figs. 4.6-1 and 4.6-2 respectively], and (2) drillholes or mine shafts that may have provided a means for escape of the radioactivity.

This repository was constructed during the period $A D 1985$ to 1995, was filled with waste from 1995 to 2020, and has been sealed in 2020. This is the first major effort by humans to attempt a long- 


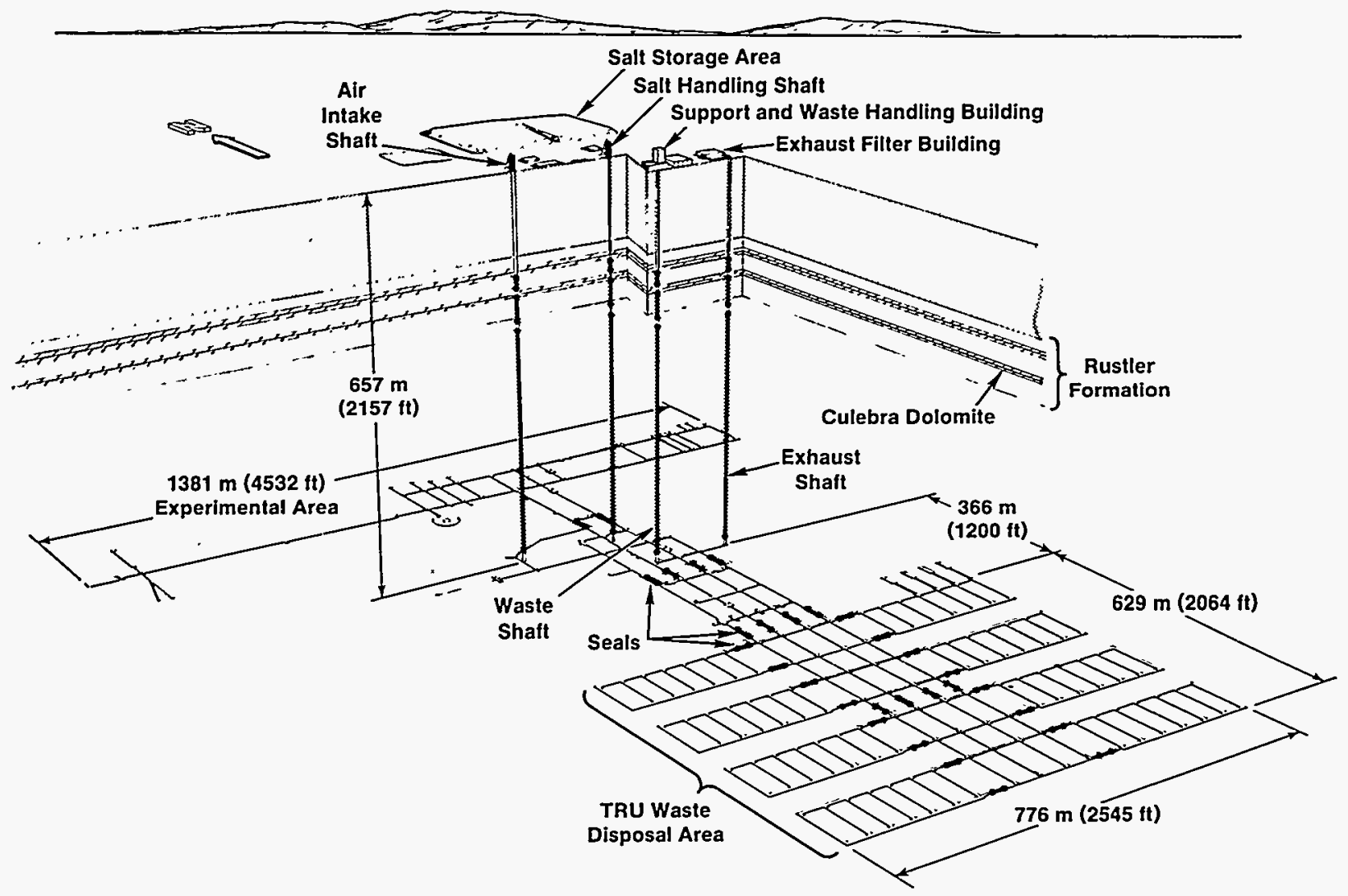

Figure 4.6-1. Detailed perspective view of the repository and site (for the Level IV chamber). 


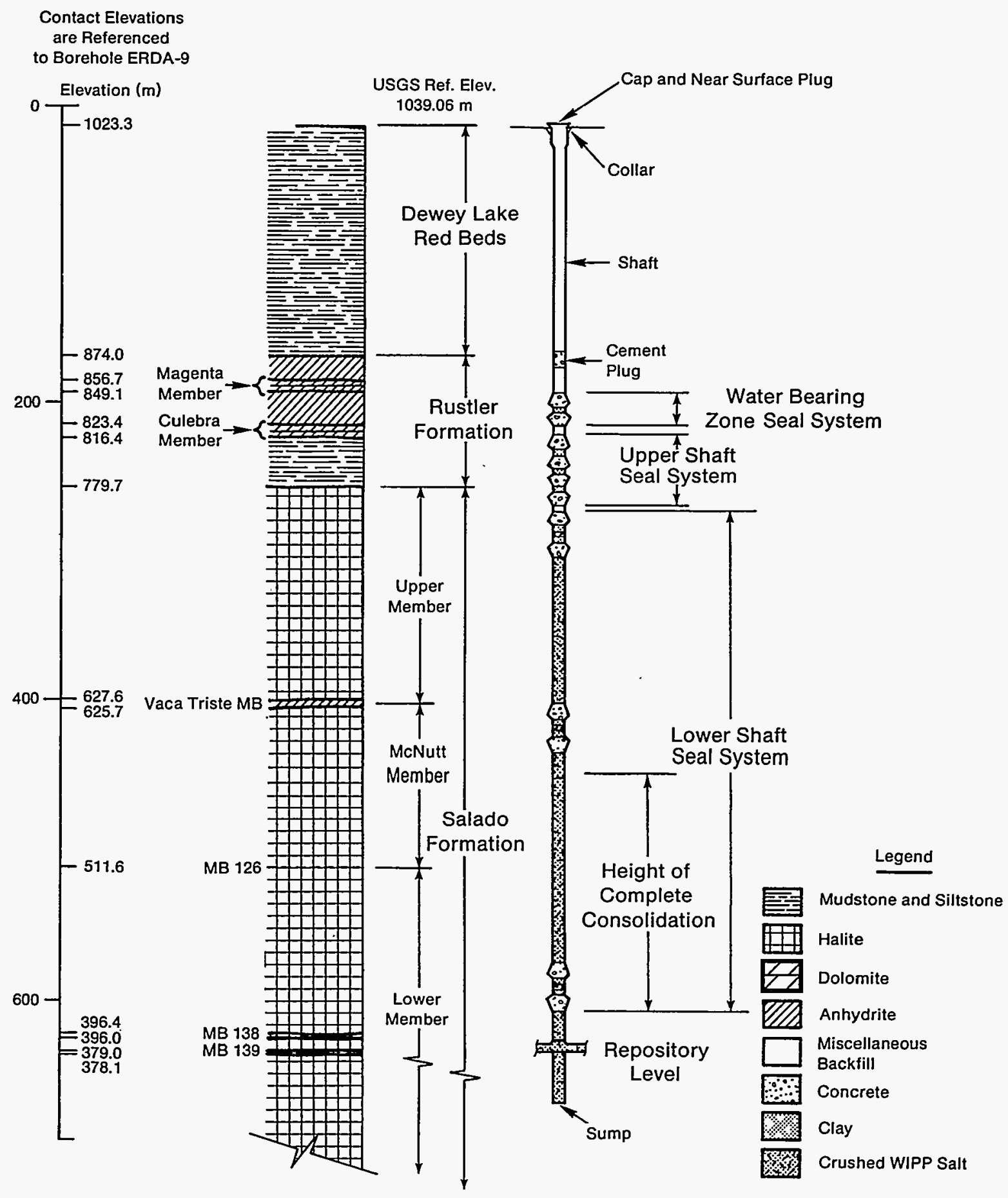

TRI-6342-311-2

Figure 4.6-2. Details of the shaft sealing (for the Level IV chamber). 
term solution to the problem of radioactive waste disposal, for we believe that we have an obligation to protect future generations from the hazards that we have created. This repository is known as the Waste Isolation Pilot Plant and has been built and operated by the government of the United States of America at a cost of $\$ x x$, which corresponds to the average annual family income of $y y$ households. At the time of its construction the United States had accumulated over a 50-year period a great amount of hazardous radioactive wastes with long half-lives. Until now these wastes have been inadequately stored above the ground or in shallow burial sites. These wastes are generated by atomic energy defense activities (i.e., nuclear weapons). [Under the present WIPP LWA, these wastes are generated by atomic energy defense activities. If the use of WIPP changes, the previous statement must be modified to reflect the wastes being accepted.] The specific wastes buried at this site are primarily from the laboratories and factories involved in the construction of nuclear weapons since 1970. The long-term radioactive wastes buried here consist of radionuclides with atomic numbers greater than 92, half-lives exceeding 20 years, and concentrations exceeding 3700 nuclear disintegrations per gram per second (a gram is the mass of one millionth cubic meter of water, and there are 3,160,000 seconds in a year, the orbital period of the earth).

The information in this room is the most detailed on the site. Other rooms identical to this one are located [exact locations given], but we urge you to keep the rooms intact and buried as they are, so that they may be preserved for future generations. If the languages and diagrams in this room are difficult for you to understand, we urge you to add new translations of our texts for the benefit of future generations. This should be done for texts in this room and throughout the site; also add new markers and other structures if necessary to maintain the marking system in good, effective condition. However, do not deface or remove the original texts, diagrams, or markers, for they will remain valuable to future persons trying to understand your own translations and additions. If you want more information than is available in this room, search in historical and archaeological libraries, museums, and archives appropriate to our time. At the time of closure of this site we are sending detailed information about this site and its contents to many major archives around the world.

The site for this repository was selected and approved in a technical and political process that involved a search for suitable sites and extensive testing. Deep burial in salt beds is considered at this time to be the most economical safe disposal method for long-term radioactive waste. Other methods that have been considered include deep-well injections, placement under the seabed or inside glaciers, sending into outer space, and transmutation of the radionuclides into stable elements. The salt bed at this site at a depth of $650 \mathrm{~m}$ is about 220 million years old and is considered very stable on a time-scale of 10,000 years against geological events such as earthquakes and 
volcanism. Diagram 3 [see Fig. 4.6-3] shows the geological strata at this site and the location of the depository. Salt is considered a good medium for the permanent storage of these wastes because its presence indicates a lack of circulating groundwater, it is easy to mine, and it is mobile in the sense that it relatively quickly seals any fractures or voids, such as those of a waste repository. The site is also considered acceptable in that few resources attractive for extraction are known in the vicinity (at least at the present time or in the foreseeable future). The main such resources known in this region are potash and some natural gas. The site also is not associated with any potable aquifer (the nearest river is about $30,000 \mathrm{~m}$ $=30 \mathrm{~km}$ away) and has a very dry climate (0.3 $\mathrm{m}$ of rain per year); moreover, we expect the climate to remain dry over the next 10,000 years. The region including the site is sparsely populated and is expected to remain so (the nearest city is Carlsbad $40 \mathrm{~km}$ to the west, with a population of 25,000). The only significant uses of the region's land presently are potash mining (for fertilizers) and cattle grazing (for meat).

The repository as constructed consisted of a series of rooms carved out of the salt, each about 10-m wide by 4-m high by 110-m long. The rooms covered a total area of almost $600 \mathrm{~m}$ by $800 \mathrm{~m}$ and were accessed by a waste shaft of dimensions $x x m$ by yy $m$, Diagram 1 [shown in Fig. 4.6-1]. Other shafts were for removal of salt, and for air intake and exhaust. The radioactive wastes were brought to this site from about 15 places around the United States, some as far as $2500 \mathrm{~km}$ away. They were transported by trucks carrying specially designed containers able to withstand extreme collision and fire in the event of accidents. These containers held the waste in many steel barrels. Altogether 845,000 barrels, each of volume 0.2 cubic meters, were brought to this site in about 20,000 truck shipments. The average mass of a barrel is $x x$ grams. The barrels contain mostly ordinary items that became radioactive at some stage in the developing, testing, constructing, and renewing of nuclear weapons. Buried items include rags, clothing, bags, and containers; these are made of fabrics, plastic, glass, and metal. There are also complex machines such as motors, hand tools, and machine tools. About 60 percent of the radioactive waste also contains hazardous chemical wastes such as lead, cadmium, chromium, barium, methylene chloride $\left(\mathrm{CH}_{2} \mathrm{Cl} 2\right)$, and toluene $\left(\mathrm{C}_{6} \mathrm{H}_{5} \mathrm{CH}_{3}\right)$. Most of the radioactive waste has minimal emissions of gamma rays, but about 3 percent has enough gamma-ray emission that it had to be remotely handled at all stages, with humans well removed from the barrels. The estimated amount at the time of burial of the major radionuclides buried here is: neptunium-237 (yy grams, each with $x x$ nuclear disintegrations per second, half-life of 2,100,000 years), plutonium-238 ([same kind of information...]), plutonium-239 (...), plutonium-240, americium-241, americium-243, curium-244, uranium-233, and thorium-229 [list needs checking]. We estimate that after 10,000 years the total number of disintegrations in the buried waste here 


\section{Generalized Geologic Cross Section}

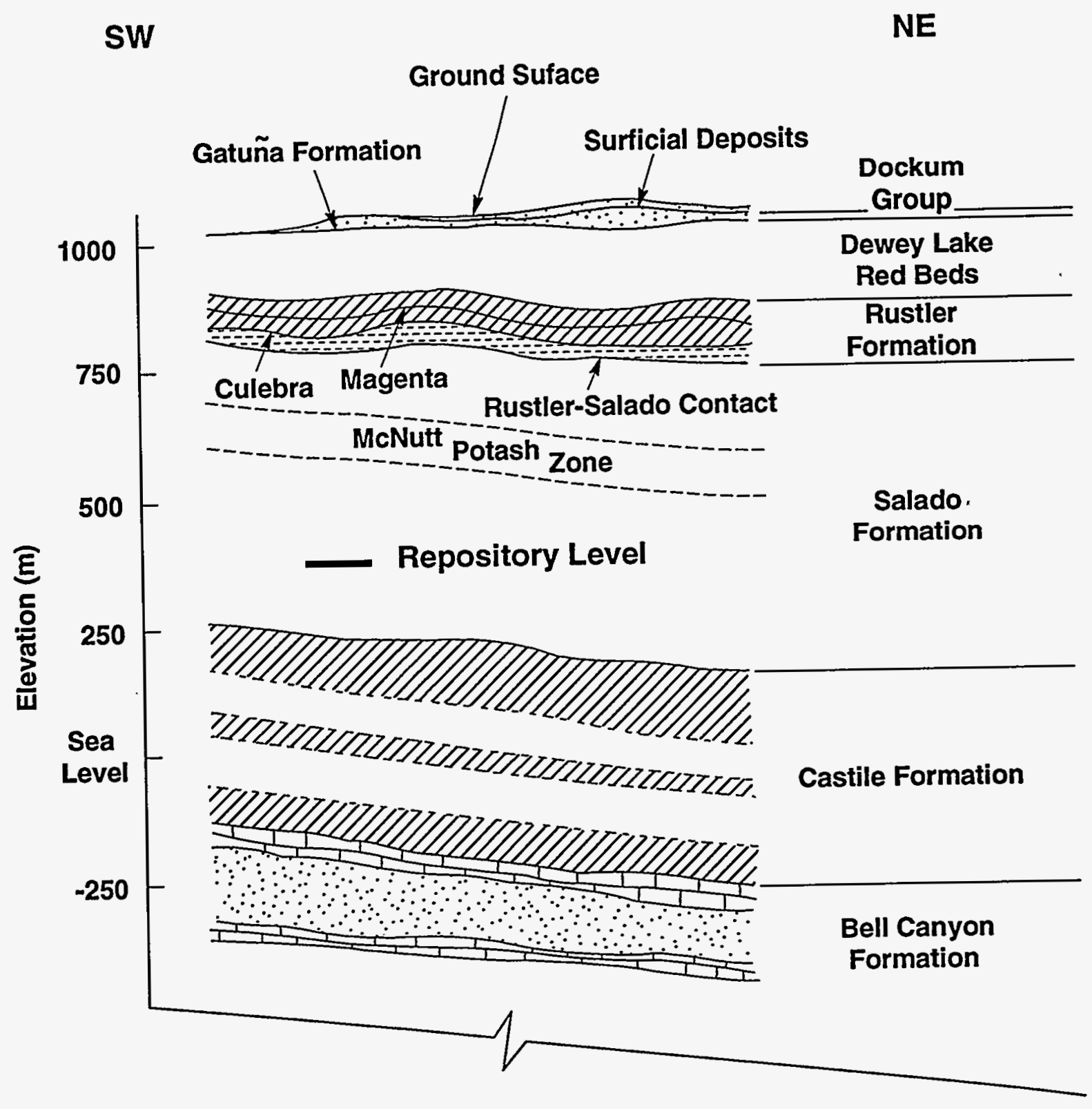

$\because \because 9$ Sand and Sandstone
$\square$ Siltstone and Sandstone Anhydrite
$\square$ Halite
Mudstone and Siltstone

Figure 4.6-3. Detailed geologic cross-section of the site (for the Level IV chamber). 
will be reduced to $x x$ per second, which means that someone standing next to this waste would encounter a level of radioactivity corresponding to yy percent of the natural background at the surface, or about the amount corresponding to typical uranium ore [see $50 \mathrm{FR} 38071$ ]. Diagram 4 [shown in Fig. 4.5-9] is a periodic table of the elements, with the radioactive elements indicated by the international "radioactivity hazard" symbol [actual symbol goes here in the text] that has been used in our time since 1950. Elements with a large amount of radionuclides buried here are also marked with a second international symbol [a filled square in the present figure, actual symbol goes here in the text] that means "radioactive waste buried here"; these symbols are then connected by lines to the repository, see Diagram 1 [shown in Fig. 4.6-1]. These two symbols have also been used widely elsewhere in our marking system. Non-radioactive, chemically toxic elements buried here are indicated with a downward-pointing circle.

After each room was filled with barrels of waste, the remaining space was then completely filled with salt. Groups of seven rooms were each sealed with a 20-m thick series of layers of cement, salt, and bentonite. Upon complete filling of the repository in AD 2020 each of the four shafts to the surface was sealed with an elaborate series of materials, Diagram 2 [shown in Fig. 4.6-2], topped by a concrete cap $x x m$ thick [give more details here of sealing, useful to the future engineer trying to fix or improve the seall. The waste rooms are expected to collapse from the weight of rock above them within 100 years and the steel barrels will break. But the salt is expected, based on our tests, to prevent the radionuclides from escaping to the surface; the expected outward diffusion into the salt is only yy meters per year. We believe that the greatest possibility for radionuclides to make their way to the surface is through human intrusion, and hence we have designed and built this elaborate marking system to warn you of the dangers. DO NOT DRILL OR DIG AT THIS SITE, DO NOT DO ANYTHING ELSE THAT MIGHT DISTURB THE WATER OR ROCKS IN THIS AREA. We believe that the most likely type of accidental intrusion is drilling a hole that penetrates both the site and the salty water found at some levels above and below the repository. This water may then become contaminated and reach the surface through the drillhole Igive specific data here].

We have found it extremely difficult to imagine all the forms of human society and available technology that over the next 10,000 years might give people the 
desire and ability to intrude into the repository level and thus potentially bring great harm to themselves. Nevertheless, we have done the best we can in the design of a marker system that will survive over this period, that will be understood by those who encounter it, and that will be effective in countering their natural curiosity to dig at such a uniquely marked and fascinating place. We have considered the options of not marking the site at all, of trying to pass on through social institutions the vital information about this site, and of building a barrier that would physically prevent intrusion by future generations. All of these have been found wanting in important ethical or practical ways, and so we have built this passive marker system. We have designed the overall appearance of the site to deliver the desired message at a psychological level, for we believe that our distant descendants will probably share with us far more psychology than technology. This desired message is "extreme danger to your health if you drill or dig here; this message is valid for a very, very long time; there is nothing valuable buried here, only dangerous material." $A$ detailed map of the marker system is given in Diagram 5 [not shown-determined by final design of marker system]. [When final design is chosen add here appropriate sentences describing the physical layout, including subsurface markers.] The alignments shown on the map toward the azimuth angles of 110 degrees, 160 degrees, 66 degrees, and 42 degrees correspond to the locations where the four brightest stars now visible from this site rise: Sirius, Canopus, Arcturus, and Vega. Because of precession of the poles, these star-rise locations constantly change and thus a measurement of these alignments allows an accurate dating of this site.

In order to increase the chances of successful transmission, the details of the message have been given many different, redundant forms, in materials, locations, languages, graphics, and amount of detail. Most common is the approximately 15-word basic message flanked by two human faces [shown in Fig. 4.5-4], which we believe will carry for distant future generations the same effect as for us. The one on the left is of horror and terror [actual face goes here in the text], and on the right is one of disgust [other face goes here]. [Level II and Level III messages will also be found in the Level IV chamber and thus do not need to be repeated here.] In this message the international "radioactivity hazard" symbol [actual symbol goes here in the text] is also introduced by placing it next to the word "radioactive" with an arrow below it pointing downwards, to indicate that the radioactivity is below the ground, not on the surface. This and all other messages are given in the following six languages, which are the official languages of the United Nations organization and are the native tongue for about 40 percent of the world's population of 5,100,000,000 persons [1988 figure]: Chinese, Russian, English, Spanish, Arabic, French. We also give these messages in Navajo, that is the native American language with the most widespread literature, and corresponds to an indigenous people who live about $700 \mathrm{~km}$ to the northwest of this site. 
The next type of message [Level III] is engraved less frequently on the site and is more detailed than the basic message described above, but still does not assume any scientific knowledge about radioactivity. It is flanked by two diagrams. The one on the left is a perspective, scale view of the repository in relation to the surface and its marking system; this is a simpler version of Diagram 1 [shown in Fig. 4.6-1]. It also shows with an arrow where the reader is located. The right diagram [similar to Fig. 4.5-8] shows the path of the north celestial pole through the sky due to the precession of the earth's axis of rotation. Bright stars are indicated by circles (the brightest star, on the left, is Vega) and portions of our constellations Ursa Minor, Draco, and Cygnus are shown by dashed lines connecting stars. The illustrated section of arc corresponds to the period from $A D$ 2020, when the pole was close to the star Polaris and the repository was sealed, to $A D$ 12,000, when the pole will be in Cygnus. The faces at the two epochs express differing emotions about the safety of intruding into the repository, and the sequence of "radioactive waste buried here" symbols [actual symbol goes here in the text] of diminishing size expresses the diminishing amount of radioactivity present in the repository as 10,000 years pass. The level of radioactivity in the waste decreases over time, but it will not all be gone after 10,000 years. If you have accurately observed the changing position of the pole in your own time, this diagram shows you how to determine the date of the sealing of this repository reasonably accurately even if you do not understand the " $A D$ " (Anno Domini) notation used for Gregorian calendar dates in this message. In other calendars of our time, the end of the year $A D 2020$ occurs during the following years: 7529 in the Byzantine calendar, 5781 in the Jewish calendar, 1441 in the Islamic calendar, and 4718 in the Chinese calendar. It also occurs on Julian Date 2459275.

This radioactive waste repository and marker system is in fact only one of many constructed all over the earth; Diagram 6 [Fig. 4.5-5] shows a map of the world (in a two-dimensional projection of the globe that preserves the correct relative sizes of all areas) with the waste sites indicated by the "radioactive waste buried here" symbol [actual symbol goes here in text]. In order to locate these sites more accurately, each symbol on the map has been labeled with a number that can also be found labeling two dots found in Diagram 7 [shown in Fig. 4.5-6] (whose most noticeable feature is a 3-m diameter circle). Each dot on the circumference of the outer circle gives the longitude of another waste site relative to the longitude of this site; this relative longitude is equal to the arc traversed from the dot labeled 0 at the top (which corresponds to this site). Dots to the right represent sites to the east. In a similar manner, the in- 
ner partial circle gives the relative latitude of a site, which is equal to the arc traversed from the dot labeled 0 . Dots on the upper side are sites to the north. The fabrication of these panels [accuracy of $1 \mathrm{~mm}]$ has been such that we believe that you can determine the location of all other radioactive waste sites from our time to an accuracy of about $4 \mathrm{~km}$. We urge you to check these locations around the world and make certain that the marking systems for these sites are still intact. You will also find that certain features of these other marking systems are identical to those here. The international standard for these marking systems can be summarized thus [the standard given here is only an example]:

"Each site must (1) display its basic warning messages in at least the following languages: Chinese, Russian, English, Spanish, French, and Arabic; (2) prominently display the symbol for international radioactive waste burial [symbol goes here]; (3) display in a protected chamber a world map of all disposal sites, together with a standard diagram that geometrically allows their location to an accuracy of at least $5 \mathrm{~km}$; and (4) include earthen berms to delineate the disposal area with heights of least $10 \mathrm{~m} . "$

\subsection{Additional possible components}

\subsubsection{Art}

Art is one of the basic ways that humans express themselves and is therefore a candidate for inclusion in any message system designed to span ten millenia. In this section, we refer to the incorporation in the site design of a specific work of art by an artist who is commissioned to create a piece that will pass on the basic message of "Danger - do not dig here."

Examples of artists whose work may be relevant include James Turrell (Roden Crater near Flagstaff, AZ), Charles Ross (Star Axis near Las Vegas, NM), and James Acord ("Monstrance for a Grey Horse'" in Richland, WA). The first two artists have specialized in sculptural pieces using light, and now are involved with large earthwork projects with astronomical connections. The third is a sculptor who uses in part radioactive materials and who is now closely working with engineers and scientists at Hanford.

We see a prominent site-specific work of art as a potentially valuable component of a marker system. But to reduce ambiguous interpretation, it should be only one design element of the overall, redundant marker system. Furthermore, any work of art should be an integral, permanent part of its milieu, thus lessening the desire (or even ability) of future museums to haul it away. 
Appendix F: Team A Report

\subsubsection{Aeolian Structures}

Communication of the basic Level I message could also take place through sound. Although probably not lasting for the full 10,000 years, structures designed to resonate in the wind could be placed around the site. The effect of the various sounds generated should be consonant with the overall site design, namely a place of great foreboding. Indeed, sounds that can readily be generated by long-lasting aeolian structures turn out often to be dissonant and mournful, and so would readily serve our purposes. Noise levels would need to be controlled so as not to disturb people residing in the general vicinity of the site.

We have not been able to research this idea further, but it deserves attention; for if it is feasible, it would be of great utility for at least the first few hundred years.

\subsection{References}

In addition to electronic data bases (INSPEC, COMPENDEX, and RAPRA), the following references were consulted in the preparation of Section 4.4:

[4-1] Levine, A.G. 1982. Love Canal: Science, Politics, and People. Lexington, MA: Lexington Books, D.C. Heath and Company.

[4-2] Biczók, I. 1968. Betonkorrosion-Betonschutz. 6th ed. Wiesbaden: Bauverlag GmbH.

[4-3] American Concrete Institute. 1975. Durability of Concrete. ACI Publication SP-47. Detroit, MI: American Concrete Institute.

[4-4] Swift, P.N. 1993. "Long-Term Climate Variability at the Waste Isolation Pilot Plant, Southeastern New Mexico, USA," Environmental Management. SAND91-7055. Vol. 17, no. 1, 83-97.

[4-5] Fujita, T.T. 1978. A Site-Specific Study of Wind and Tornado Probabilities at the WIPP Site in Southeast New Mexico. Satellite and Mesometeorology Research Próject (SMRP) Research Paper No. 155. Chicago, IL: The University of Chicago.

[4-6] Bagnold, R.A. 1941. The Physics of Blown Sand and Desert Dunes. New York, NY: William Morrow \& Company.

[4-7] "Light Earthquake Sends Tremor Through Texas and New Mexico," New York Times. January 3, 1992, Section A, p. 14, col. 2.

[4-8] Neville, A.M. 1963. Properties of Concrete. New York, NY: John Wiley \& Sons, Inc. 
[4-9] Torres, L. 1985. "To the Immortal Name and Memory of George Washington": The United States Army Corps of Engineers and the Construction of the Washington Monument. EP 870-1-21. Washington, DC: Historical Division, Office of Administrative Services, Office of the Chief of Engineers.

[4-10] Hawkes, J. 1976. The Atlas of Early Man. New York, NY: St. Martin's Press.

[4-11] U.S. Department of Energy. 1979. Environmental and Other Evaluations of Alternatives for Long-Term Management of Stored INEL Transuranic Waste. DOE/ET-0081. Washington, DC: U.S. Department of Energy, Assistant Secretary for Energy Technology, Office of Nuclear Waste Management.

[4-12] Craig, B.D. 1989. Handbook of Corrosion Data. Metals Park, OH: ASM International.

[4-13] Baboian, R., and S.W. Dean, eds. 1990. Corrosion Testing and Evaluation: Silver Anniversary Volume. STP 100. Philadelphia, PA: ASTM.

[4-14] Baloun, C.H., ed. 1990. Corrosion in Natural Waters, STP 1086. Philadelphia, PA: ASTM.

In Section 4.5, we refer to the following references:

[4-15] Eibl-Eibesfeldt, I. 1989. Human Ethology. New York, NY: Aldine de Gruyter.

[4-16] Metropolitan Museum of Art. Photograph Library. Accession nos. 26.7.1020,.1021. New York, NY. 
Appendix F: Team A Report 


\section{Appendices}

\subsection{Scenario for the marking system (MFK)}

Jo and Steve bumped along comfortably as Jo steered the drilling rig over the undulating desert terrain. The sun was just up over the horizon, but the day had not yet grown hot. The sky was a clear, dark blue with no clouds, and the color contrasted sharply with the tans and reds of the desert. There were sand dunes, some free-form and mobil, others quietly building up against the mesquite trees. Steve checked the computer screen. "We ought to be in sight by now," he said.

There were no tracks, so Jo just followed the terrain and the navigation system on the computer. She wrestled the vehicle up over a small dune. "There she is!" cried Jo. "Looks just like the aerial shot. The funny thing is, the aerial shot looks more like a drawing than a rock formation. This is going to be a strange place." She turned the drilling rig slightly and headed directly toward the strange shape to the north.

The shape turned out to be a series of jagged-shaped earthworks slowly growing higher as they moved toward a center. But they didn't meet in the center. From a distance, the top looked flat, but little bits of blue told them that there were passageways through the hills.

"Let's take another look at that aerial," said Jo. Steve brought it up on the screen. Jo stopped to give it a good look. From the air, the place looked like a series of lightning bolts streaking away from an empty center. The center was also where Remote said they got a very strong, but unrecognizable, signal on their recent survey. "Strange," commented Steve. "It sure doesn't look natural."

"I don't care if the Martians built it," replied Jo. "I'm just a tool pusher and I'm due to go home next week. Let's go."

They drew closer, and Jo swung the rig so it followed the winding path between two hills. She hit the brakes hard, and the two of them snapped against the belts. Blocking their way was a rock. It was right in the middle of the path and the rig couldn't fit around it.

Steve hopped out and looked around. "Well, I'll be," he said, "It's not rock! It's some kind of concrete!" He followed the shape around and disappeared for a few minutes. His head poked out from behind the shape. "Jo," he hollered, "Come and look at this!"

Jo climbed down and walked over, the sand squeaking beneath her boots. She followed Steve's head around the shape, only to find another shape behind it. The sand had shifted in between the two, but the writing was still clear. She stood next to Steve and looked. There were faces, two of them. And there was writing, in many different forms. "Hey, I think that's Chinese -- I saw something like it in my ancient history class," said Steve as he knelt to get a closer look. 
"So send a picture to Cindy in Remote -- you know she likes that old stuff," shrugged Jo. "What did they want around here? Those faces aren't scary. That one looks scared and that one looks sick. I'm not impressed." She stepped out between the pillars and looked around. They had stopped just before the center. There was nothing there but sand and scrub. She squinted and saw that every passage way was blocked by these little shapes sticking up in the middle. She sighed. All this stuff for... nothing? That wasn't her problem; her problem was how to get the rig in there and get the core. The sooner she was done, the happier she'd be. She had begun to dislike this place.

Jo turned to go back to the rig and think. If she blasted the shapes, it wasn't clear the path would be passable. The walls would stop the rubble from traveling very far. Should she pull them or go with a directional hole? She stopped to stare at the shapes. No telling how far down they may go. The rig was designed for drilling. It could pull 10,000 rangs of pipe, so it could probably handle the shapes. There was no sense risking damage though; the directional hole was probably the safest route to take.

Steve was already back at the rig. He really had sent a shot of the place to Cindy. Jo shook her head. "When you're done playing, I need to reprogram the rig." Steve moved aside. "So we are going to start here?" "Yup," was the reply.

Steve went to the other set of controls. He set out the bracing legs to stabilize the rig. He activated the roustabout robots that would join the lengths of pipe as the drilling proceeded. He prepared the casings to store and transport the geological cores back for analysis. He checked the air system. Air was the fluid of choice for drilling systems now, no need to locate a water source near the drill site.

Jo was checking in with Headquarters. "Yeah, rather than a straight hole, just a 5-sec deviation will still bring me right where you want it at 2,100 rangs. The system has been reprogrammed and no difficulties are indicated. All we are going through is some shale and salt, till we get to the interesting stuff. Any problem?"' Jo waited until clearance came through.

She swiveled around, put on her hearing protectors, and began. Steven had everything ready. She spudded the hole and watched as the cuttings blew to the discharge pile. The drill bit cleanly through the beginning layers. She fine-tuned the bit for the salt she expected to hit.

When things were underway, they put both systems on automatic and ate dinner. "Looks ok, so far," said Steve. "Yeah," replied Jo, munching on a biscuit. "With luck, we'll be at 2,100 rangs by tomorrow morning." Steve took the first watch while Jo curled up for some sleep.

Steve nudged her. "Your turn. Things are going so well; it is boring. Can't imagine what's causing the signal the Remote is so interested in." Jo got up, grabbed some coffee and looked 
over the controls. The depth was 1,800 rangs. She gently increased the air pressure to keep the hole open at that depth.

Steven had just fallen asleep when the alarms blew. "What the?" he cried. "We lost the bit!" shouted Jo, trying to regain control. She swore. "We've got a stuck pipe and I'm afraid it may have snapped. We may have to go fishing."

Steven got out and checked the last core as it was coming up. There were rangs and rangs of salt. What could have happened, he wondered. The last section was just coming into view. After the robots had laid it down on the rack, he shut them down too. He looked over, and gave a low whistle. He walked back over to Jo who was still bent over the screen. "Nothing makes sense," she was mumbling.

"What happens if you run a salt bit into hard rock at 100 rangs per hour?" Steve asked sweetly. "That'd be a stupid thing to...." Jo picked up her head, "Huh?" "Well, that's what's out there." Steven held out his hand. In his palm lay a chuck of red granite.

Jo didn't have time to reply. The communicator squawked and, rubbing her eyes, Jo punched it to answer. "Rig 3 here." The face of her boss showed on the screen. "Stop work immediately. That's an order!" stated the dark face on the screen. "You're too late, we've already stopped," replied Jo. Her boss stiffened in her chair. "Since when do you read ancient Chinese?" asked Linda. "I don't," replied Jo. "I've just lost a bit. What's all this about?"

Linda looked worried. "What broke the bit? What level are you at?" she queried. "I hit hard crystalline rock at 2,100 rangs. God knows, that alone won't give the signal Remote found, but I'm going to have to fish everything out, and put on the spare before I find out." Jo didn't like explaining she lost the bit.

Linda looked relieved. "Good. You didn't go any deeper." It was Jo's turn to stiffen. "What is going on here? If I don't complete the job, I don't get paid.”

Linda smiled. 'Don't worry. You'll be paid in full. This is an official job change. The data Steve sent to Cindy were most interesting. We know what the signal is now, so you won't have to drill. It's an old waste disposal site like the one they hit 10 years ago in the north mountains."

Jo shuddered. She had heard about that site. Another crew went exploring. In that case, the stuff was much closer to the surface and they lost a couple of people before they figured things out. She liked her job and it paid well, but it did have its risks. "So just close up shop and go home?" she asked. "That's right," said Linda as she signed off.

Steve and Jo reloaded all the equipment and got ready to back out of the passageway. But before she left, Jo walked over to the shapes again -- the ones that blocked their way to the dead zone. She looked again at the faces. They were right, she thought. As she climbed into the $\mathrm{cab}$, she told Steve "Let's get out of here. I knew I didn't like this place." 


\subsection{The enormity of marking the WIPP site (FN)}

If the WIPP is ever operational, the site may pose a greater hazard than is officially acknowledged. Yet the problems involved in marking the site to deter inadvertent intrusion for the next 10,000 years are enormous. Even if knowledge exists that would allow translation of the message on the markers, there might be little motivation to solicit such knowledge. Pictorial messages, however, are unreliable and may even convey the opposite of what is intended.

This panel member therefore recommends that the markers and the structures associated with them be conceived along truly gargantuan lines. To put their size into perspective, a simple berm, say $35-\mathrm{m}$ wide and $15-\mathrm{m}$ high, surrounding the proposed land-withdrawal boundary, would involve the excavation, transport, and placement of around 12 million $\mathrm{m}^{3}$ of earth. What is proposed, of course, is on a much grander scale than that. By contrast, in the construction of the Panama Canal, 72.6 million $\mathrm{m}^{3}$ were excavated and the Great Pyramid occupies 2.4 million $\mathrm{m}^{3}$. In short, to ensure probability of success, the WIPP marker undertaking will have to be one of the greatest public works ventures in history. 


\subsection{Personal thoughts (WS)}

Working on this panel, always fascinating and usually enlightening too, has led to the following personal thoughts:

(a) We have all become very marker-prone, but shouldn't we nevertheless admit that, in the end, despite all we try to do, the most effective "marker" for any intruders will be a relatively limited amount of sickness or death caused by the radioactive waste? In other words, it is largely a self-correcting process if anyone intrudes without appropriate precautions, and it seems unlikely that intrusion on such buried waste would lead to large-scale disasters. An analysis of the likely number of death over 10,000 years due to inadvertent intrusion should be conducted. This cost should be weighted against that of the marker system.

(b) The design and testing of markers and messages must involve a broad spectrum of societies and people within those societies. So-called "experts" can of course make important contributions, but they must listen carefully to all other people who represent those who might encounter the markers. In the course of working on this project, I received excellent ideas from a wide range of undergraduates, colleagues, friends, and relatives.

(c) The very exercise of designing, building, and viewing markers creates powerful testimony addressed to today's society about the full environmental, social, and economic costs of using nuclear materials. We can never know if we indeed have successfully communicated with our descendants 400 generations removed, but we can, in any case, perhaps convey an important message to ourselves. 
Appendix F: Team A Report

\subsection{Possible origins of archetypes of place (MB)}

Several explanations are offered for the phenomenon of archetypes of place. All receive some external validation in various literatures and all (or some) may be operating simultaneously.

\subsubsection{Landscapes Seen as Having Adaptive Value in Evolution}

Much current theory about our strong and stable preferences for particular forms of landscape and habitat sees them as adaptive behavior. It sees the common feelings, meanings, and preferences people have in regards to types of places as a product of our bio-evolutionary history of successful adaptation in certain habitats.

Landscape archetypes may be so powerful because they were "imprinted" over an incredibly long period of time (clearly far longer than we have had cultures and built-form); imprinted during the period of the mind's greatest openness to landscapes, during the development of consciousness; imprinted at a time of our fullest sensory integration, and in a situation of our most profound participation in nature seen as a life-unity. Some theories suggest that landscape archetypes originate in the physiologically nurturing habitat of our evolutionary "cradle," the African savannah, which provided ample food, water, breeding grounds, and cover/refuge, all requirements for survival. Humans who prospered were those who preferred the savannah as habitat, while those who preferred other and less salutary habitats did not survive. These adaptive preferences either were or became "hard-wired" and genetically transmitted, so that these landscape preferences remain with us today, even though there is no lingering survival value ([Ref. 5-1], [Ref 5-2], [Ref. 5-3], [Ref. 5-4], [Ref. 5-5], and [Ref. 5-6]).

Another research supported theory is about the survival value of an enhanced ability to read and know environments so we may more wisely bend them to our purposes. Appleton [Ref. 5-1] posits three types of cues in the landscape (hazards, prospects and refuges) with which wise cue-readers would be rewarded with enhanced chances of survival. Hazard cues, when perceived, arouse anxiety that is resolved when some successful action is taken, leading to relaxation and even pleasure. Because some or all parts of this "hard-wired" sequence have had adaptive value, we still display strong preference for environments that provide a good balance of prospect and refuge, even though it is no longer adaptive.

The theory of understanding and exploration of landscapes of S. Kaplan ([Ref. 5-7], [Ref. 5-8], and [Ref. 5-9]) is broader, and emphasizes the evolved ability to read, understand and explore the landscape. The corollary is that we still prefer landscapes that are recognizable, invite some exploration, and are comprehensible and interpretable.

Orians and Heerwagen [Ref. 5-6] link several landscape and habitat preference theories in their concept of a three stage interaction process, much of which is run "on automatic," on evolved and imprinted responses. The stages are (1) a rapid emotional response to physical qualities of an environment; (2) information-gathering, engaged by features that entice exploration, aid it, 
and support wayfinding, especially " the way back"....all helped by an automatic risk-assessment; and (3) the decision to inhabit (or not) based on the presence of "patches" of things needed for survival and available with reasonable energy expenditures.

Some researchers also argue that our archetypes for built-form are based on those for landscapes (Hildebrand [Ref. 5-10]).

\subsubsection{Landscapes as Primordial Factor in Development of Mythic Consciousness}

In his analyses (done in the twenties and published 1955 and 1973) of the development of human symbol formation ([Ref. 5-11] and [Ref. 5-12]), Cassirer locates its origins in our mythic consciousness, where the mythologies of peoples are not the products of consciousness, but are the imprinted evolutionary "record" of the history of the development of consciousness itself...the idea that myths really took place in consciousness during its long development. Archetypes (of place and all else) reside in the unconscious, made from primordial material over an enormous time. McCully [Ref. 5-13] suggests that the primordial materials in the unconscious are "prototypical experiences of food gathering, elimination, fertility, father, mother, authority; self, femininity, goddess, eternity, childhood, circle, square, devil (evil), god (good), maleness and sleep." To these Cassirer would certainly add "space, time, and number" and I would add "communion; community; body-danger; pain and death." These may be considered the substratum, the basic materials of human experience and meaning, and humans explore and represent these primordial materials in all our symbolic forms: myth, language, religion, and art.

As an example, there seems to be a world-wide set of common myths, ones that have near-identical basic structure and that only differ in details. While we only see local or what Joseph Campbell [Ref. 5-14] calls "ethnic variations," and never see the archetypal myth at the center, the remarkable structural commonalities attest to that archetypal center and meaning. Some species-wide mythic themes are: the creation of the world from a chaos of nothing; the fire-theft; the great mother; virgin birth; the plenitude of Eden and the beauty of paradise; the chaos-again of the flood or deluge; the land of the dead; the dying and resurrected god or hero; the questing journey or pilgrimage; and redemption through sacrifice and suffering.

The fundamental human experiences carried as archetypes are ones that, when experienced "in the beginning," already had a mythically significant "tone." In fact, our predisposition to even notice certain things and not others is because they have some meaning...they first "appear" to us as significant, against a background of all else, which at that moment, seems irrelevant. This experience, Eliade [Ref. 5-15] argues, is the origin for the fundamental articulation of the Sacred and the Profane. 
While it is, of course, our projection of meaning onto a world, it seemed and still seems like a perception of meaning in the world. As Cassirer [Ref. 5-12], the philosopher of symbolic form, said in Language and Myth, in 1923:

"The mythical form of conception is not something super-added to certain definite elements of empirical existence; instead, the primary experience itself is steeped in the imagery of myth and saturated with its atmosphere."

It is this experiencing-as-significant, this irruption of meaning, which forms the basis for the development of, first, concepts, and then the early symbolic forms of myth and language. And it is these significant meanings that are also embedded in the unconscious in yet another symbolic form, that of archetypes.

\subsubsection{Archetypes of Built-Form Seen as Originating in Body-Experience}

Some theories about our feelings and preferences for built-forms suggests primary origins in the body.

Harries ([Ref. 5-16], [Ref. 5-17], and [Ref. 5-18]) uses the term "natural language"' to describe how the body senses itself in a place and makes sense of a place, while moving through it and using it. This "language" is derived and transmitted through millennia of these common experiences. He posits fundamental dialectics as "natural symbols" in human experience: our bodies' six axial directions and its center, and the polarities of phenomena related to vision, hearing, touch, gravity, and location (dark-light, loud-soft, rough-smooth/hard-soft/cold-hot, heaviness-lightness, here-there/inside-outside). His work shows that in all spatial experience, the body feels and responds to these, and there is meaning. Much current research in the phenomenological meanings of places supports this.

Walter [Ref. 5-19] and others posit "haptic perception" in which the body feels the articulations of shapes and surfaces in the world by means of its own inner articulations, and (almost literally) "grasps" meaning from form.

Thiis-Evensen [Ref. 5-20] in a work called Archetypes in Architecture links body-feeling more directly to the primary physical elements used in making buildings. From a fundamental dialectic of the balance of the forces of inside and outside come the archetypal physical elements that delimit spatiality: the wall, floor, and roof (and further, the door, window, and stair), and their activity in mediating between inside and outside. It is our body that senses the meanings through our relationship to three aspects of each element: motion (its dynamic nature...felt as expanding, contracting or balanced); weight (its relation with gravity); and substance (the character of material...hard/soft; warm/cold).

There are many others who have studied how we comprehend the meanings of place through our bodies' posture, orientation, feel, and movement, such as Yi Fu Tuan in Space and Place, The Perspective of Experience [Ref. 5-21]; Kent Bloomer and Charles Moore in Body, Memory, and 
Architecture [Ref. 5-22]; Joseph Grange's "Place, Body and Situation" in Dwelling, Place and Environment [Ref. 5-23].

There are other possibilities. I will not describe them here, but important ones are: Bachelard [Ref. 5-24], Condon [Ref. 5-25], Lobell [Ref. 5-26], and Munro [Ref. 5-27].

\subsubsection{References}

[5-1] Appleton, J. 1975. The Experience of Landscape. New York, NY: John Wiley \& Sons.

[5-2] Dubos, R. 1965. Man Adapting. New Haven, CT: Yale University Press.

[5-3] Dubos, R. 1968. So Human an Animal. New York, NY: Charles Scribner's Sons.

[5-4] Dubos, R. 1980. The Wooing of Earth, New York, NY: Charles Scribner's Sons.

[5-5] Orians, G.H., 1986. "An Ecological and Evolutionary Approach to Landscape Aesthetics," Landscape Meanings and Values. Eds. E.C. Penning-Rowsell and D. Lowenthal. London, England: Allen and Unwin. 3-25.

[5-6] Orians G.H., and J.H. Heerwagen. 1992. "Evolved Responses to Landscapes," The Adapted Mind: Evolutionary Psychology and the Generation of Culture. Eds. J.H. Barkow, L. Cosmides, and J. Tooby. New York, NY: Oxford University Press. 555579.

[5-7] Kaplan, S. 1975. "An Informal Model for the Prediction of Preference," Landscape Assessment: Values, Perceptions, and Resources. Eds. E.H. Zube, R.O. Brush, and J.G. Fabos. Stroudsburg, PA: Dowden, Hutchinson \& Ross, Inc. 92-101.

[5-8] Kaplan, S. 1979. "Perception and Landscape: Conceptions and Misconceptions," Our National Landscape: A Conference on Applied Techniques for Analysis and Management of the Visual Resource, Incline Village, NV, September 1979. Berkeley, CA: Pacific Southwest Forest and Range Experiment Station. 241-248.

[5-9] Kaplan, S. 1987. "Aesthetics, Affect, and Cognition: Environmental Preference from an Evolutionary Perspective," Environment and Behavior. Vol. 19, no. 1, 3-32.

[5-10] Hildebrand, G. 1991. The Wright Space: Pattern and Meaning in Frank Lloyd Wright's Houses. Seattle, WA: University of Washington Press.

[5-11] Cassirer, E. 1955. The Philosophy of Symbolic Forms. Volume 2: Mythical Thought. Translated by R. Manheim. New Haven, CT: Yale University Press.

[5-12] Cassirer, E. 1946. Language and Myth. Translated by S.K. Langer. [New York, NY]: Dover Publications, Inc. 
Appendix F:- Team A Report

[5-13] McCully, R.S. 1987. Jung and Rorschach: A Study in the Archetype of Perception. Dallas, TX: Spring Publications.

[5-14] Campbell, J. 1959. The Masks of God. New York, NY: Viking Press. Vols. 1-4.

[5-15] Eliade, M. 1959. The Sacred and the Profane: The Nature of Religion. New York, NY: Harcourt Brace Jovanovich.

[5-16] Harries, K. 1982. "Building and the Terror of Time," Perspecta. Vol. 19, 58-69.

[5-17] Harries, K. 1988. "Representation and Re-presentation in Architecture," Via. 1988, no. $9,[12]-25$.

[5-18] Harries, K. 1988. "The Voices of Space," Center: A Journal for Architecture in America. Vol. 4, 34-49.

[5-19] Walter, E.V. 1988. Placeways, A Theory of the Human Environment. Chapel Hill, NC: The University of North Carolina Press.

[5-20] Thiis-Evensen, T. 1987. Archetypes in Architecture. New York, NY: Oxford University Press.

[5-21] Tuan, Y-F. 1977. Space and Place, The Perspective of Experience. Minneapolis, MN: University of Minnesota Press.

[5-22] Bloomer, K.C., and C.W. Moore. 1977. Body, Memory, and Architecture. New Haven, CT: Yale University Press.

[5-23] Grange, J. 1985. "Place, Body and Situation," Dwelling, Place and Environment: Towards a Phenomenology of Person and World. Eds. D. Seamon and R. Mugerauer. Boston, MA: Martinus Nijhoff Publishers. 71-84.

[5-24] [Bachelard, G.] 1969. The Poetics of Space. Translated from the French by Maria Jolas. Boston, MA: Beacon Press.

[5-25] Condon, P.M. 1988. A Designed Landscape Space Typology: A Series Design Tool: $A$ Report to the National Endowment for the Arts. Minneapolis, MN: School of Architecture and Landscape Architecture, University of Minnesota. (Copy on file at the Architecture Library, University of Minnesota, Minneapolis, MN.)

[5-26] Lobell, M. 1983. "Spatial Archetypes," Revision. Vol. 6, no. 2, 69-82.

[5-27] Munro, E.C. 1987. On Glory Roads: A Pilgrim's Book about Pilgrimage. New York, NY: Thames and Hudson. 


\subsection{A Proposal for a Visitors' Center and Memorial at the WIPP Site (FN)}

I wish to suggest that the structures proposed in Section 4 above be complemented with constructions of a very different sort, which should be located at or close to the most likely public approach to the message-bearing structures. They would include a visitors' center whose role would be in part to fulfill the standard function of such centers; in this case, explaining the history and design of WIPP and the marking system. However, the visitors' center and associated structures should also convey a serious message, one which will endow the entire site with the significance of a solemn memorial, or even a shrine. In brief, the message conveyed should be the destructive power of nuclear energy. Therefore, accompanying the visitors' center itself there might be symbols recognizable as denoting mourning, reflection, and remembrance. Symbolic gravesites, small shrines, and the like could serve this purpose.

The following paragraphs outline briefly the observations, assumptions, and predictions that have led me to put forward this idea.

1. If the collective proposals of Team A are carried out, the WIPP site will quickly become known as one of the major architectural and artistic marvels of the modern world. Quite simply, there will be no keeping people away. We owe it to these people to explain to them why WIPP was built and its overall significance. To do so adequately would require a dedicated information center; the structures themselves are not designed for this purpose.

2. An appropriate message for the public area leading to the markers is the insanity of nuclear war and the dangers inherent in the preparations for one. The principal exhibits could feature the destruction of Hiroshima and Nagasaki and the now acknowledged Soviet disaster at an atomic weapons complex in the Urals in 1957 that forced the evacuation of 10,000 people. Other exhibits could document and thereby help atone for the lack of forthrightness on the part of the government in informing the affected public about the dangers they have faced as a consequence of nuclear weapons production and testing. (A good example is the plight of the Hanford "downwinders," who now suffer disproportionate incidences of thyroid and other cancers because no one told them about the 530,000 curies of radioactive iodine isotopes that were released from the reactors between 1944 and 1956.)

Other exhibits could serve as constant reminders of the human and financial cost of nuclear power, focusing on the events at Chernobyl, Three Mile Island, and the like. I take it for granted that increasingly nuclear power will come to be looked upon as a mid-to-late 20th century folly. There is considerable reason to believe that it will be abandoned as an energy source before long. In the United States, there are many fewer power plants in operation or pending construction now than a decade ago. Many of the former are expected to be shut down and most of the latter will never be built. At the end of 1990, there were only 83 plants under construction in the world, half in Eastern Europe and not likely ever to be completed. A tragedy on the scale of Chernobyl, which is inevitable in the next decade, will end dreams of nuclear 
power as an energy source forever. Because (as we note in Section 1.3.1) it is highly likely that WIPP will be used to store civilian, as well as military, wastes, it is appropriate that the memorial at WIPP serve as a reminder of the tragic cost of nuclear power as used for "peaceful" as well as intentionally destructive purposes.

Indeed, the very existence of WIPP with its price tag of well over a billion dollars is a monument to the folly of the nuclear enterprise. We owe it to the public to explain in detail the circumstances surrounding the birth and death of this enterprise.

3. The primary task of the Marker panel teams is to devise ways to ensure that the WIPP site not be tampered with over the centuries. It seems to me that an ideal way to accomplish this would be to associate with it a memorial with solemn significance such as is described above. Obviously, no building or plot of ground is destruction-proof, but those known to bear religious, memorial, or emotional significance tend to fare better than most. By way of example, consider how difficult it is in societies around the world to expropriate cemetery land for any other purpose. There are several square miles in the borough of Queens in New York City that contain some of the (potentially) most valuable real estate in the world. Yet it is safe to say that, barring some massive cultural discontinuity, the cemeteries on this land will remain undisturbed indefinitely. In many Asian and European cities, the only standing structures more than a century or two old are temples and churches. This is due in part to the fact that they were constructed to last in a way that secular buildings were not, but also to a reluctance to destroy them. Even where the forces of history lead to one culture and its religion being displaced by another, the sacred sites of the former are often expropriated for the same purpose by the latter. The Parthenon has been successively a temple dedicated to Athena, a Byzantine church, a mosque, and (in effect) a monument to the grandeur of the ancient peoples who built it.

It is true, of course, that the conquest of one people by another is often accompanied by cultural genocide and with it the conscious elimination of the sacred symbols of the conquered. Witness the destruction of the Temple of Solomon by the Romans in $\mathrm{AD} 70$ and the systematic annihilation or removal by Christian colonizers of virtually all structures and cultural artifacts bearing religious significance among the conquered peoples in the Americas, Africa, and Oceania. ${ }^{1}$ One might have the uneasy feeling, then, that the replacement of the currently dominant "Anglo" culture by another in the New Mexico area (an event that is surely inevitable over 10,000 years) might lead to destruction of any memorial at the WIPP site. I can think of two diametrically opposed scenarios for the future involving such a replacement, an optimistic one and a pessimistic one. Neither, however, cuts at the heart of the recommendation to construct a solemn memorial at the site. In the first, optimistically speaking, there appears to be arising an historically unprecedented sensitivity to the cultural rights of the vanquished and

1 Yet the Israelis dare not rebuild the Temple because the site now bears holy significance to Islam and some religious structures in Mexico, Peru, Easter Island and elsewhere were too large for even the Western conquerors to destroy or remove. 
dispossessed. For the first time in 500 years, there is serious discussion in the dominant culture of the negative effects of Columbus' legacy. More specifically, Native American Indians have been challenging the right of anthropologists, developers, and others to continue pillaging their burial sites and removing objects of sacred value and, to a certain degree, they have been winning. For example, some states have enacted strict legislation prohibiting any kind of excavation in such sites without prior consent of the relevant Indian tribe. Thus, one has reason to hope that future political shifts in the area will leave any memorial (and the message it conveys) intact.

On the other hand, in the pessimistic scenario, any successful invader that would think nothing of destroying objects of sacred significance would also indifferently destroy simple markers, buildings, and any other objects or symbols valued by the defeated or displaced people. In such an event, any marker system would be imperiled. Therefore, there is nothing to lose by constructing a solemn memorial at the site.

4. While I am a linguist, not a physicist or a geologist, careful reading of the literature critical of WIPP ${ }^{2}$ has convinced me that it poses hazards greater than those that are officially acknowledged. I therefore feel that the site should be monitored well past the 100 -year point at which active institutional control is projected to cease. The presence of a staffed visitors' center will encourage monitoring to continue. At the same time, there would be no hazards to visitors, because the natural geological activity leading to potential public danger will be slow enough to allow more than enough time for evacuation and (hopefully) amelioration. An occupied structure near the site will also help to discourage drilling and other activity that could lead to a sudden hazardous situation.

5. Finally, let me point out that this is a particularly auspicious time to propose the kind of memorial described above. The commitment of all the major powers to nuclear disarmament should facilitate the acceptance of this idea. The Japanese, as the only country to feel the full fury of nuclear weapons, should be eager to have their experiences commemorated; the inheritors of the Soviet Union are in a period of willingly exposing their past nuclear disasters and looking for ways of defusing the arms race further; the Europeans might be expected as a matter of course to support anything symbolic of the scaling down of the arms race; and we Americans should be proud to reinforce to the world the recognition of the evil of nuclear weapons and atomic war.

2 See, for example, Don Hancock. 1989. "Getting Rid of the Nuclear Waste Problem: the WIPP Stalemate," The Workbook. Vol. 14, no. 4, 134-144; Michele Merola. 1991. "State of the WIPP Address," The Radioactive Rag. Vol. 3, no. 2, 1-2; Nicholas Lenssen. 1991. "WIPP-Lash: Nuclear Burial Plan Assailed," World Watch. Vol. 4, no. 6, 5-7; Debra Rosenthal. 1990. At the Heart of the Bomb: The Dangerous Allure of Weapons Work. Reading, MA: Addison-Wesley. 195-202. 


\section{6 "Beauty is conserved, ugliness discarded" (DGA)}

To design a marker system that, left alone, will survive for 10,000 years is not a difficult engineering task.

It is quite an other matter to design a marker system that will for the next 400 generations resist attempts by individuals, organized groups, and societies to destroy or remove the markers. While this report discusses some strategies to discourage vandalism and recycling of materials, we cannot anticipate what people, groups, and societies may do with the markers many millennia from now.

A marker system should be chosen that instills awe, pride, and admiration, as it is these feelings that motivate people to maintain ancient markers, monuments, and buildings. 


\section{Additional Information \\ From SAND92-1382 Authors}

(see p. F-24)

Unlike the containers for spent fuel, the metal containers for the WIPP waste were not designed to contribute to the isolation of the waste--they are expected to be crushed when the salt begins to creep closed and to corrode over time, producing gas. Current research is intended to answer questions about the physical couplings among room creep, gas generation, gas movement/dissipation, and brine inflow/outflow.

The closest point between the Pecos River and the WIPP is at Malaga Bend, approximately 19 kilometers (approximately 12 miles) from the edge of the WIPP land withdrawal boundary. The Final Supplement Environmental Impact Statement (January 1990) shows results from modeling the potential movement of radionuclides for a number of variations for both the undisturbed (possible migration of radionuclides from the repository to the Rustler through repository shafts) and the disturbed (possible migration of radionuclides from the repository to the Rustler through a borehole that intersects a pressurized brine reservoir below) cases. Undisturbed travel times for radionuclides from the repository to a stock well located only 3 miles south of the center of the WIPP (1 mile south of the WIPP land withdrawal boundary) ranged from 220,000 to $>4,800,000$ years. Total radionuclide concentrations in the Culebra aquifer (the formation with the greatest transmissivities within the Rustler Formation) at the same stock well only 3 miles south of the center of the WIPP at 10,000 years for disturbed cases ranged from $10^{-19} \mathrm{~kg} / \mathrm{kg}$ to $10^{-8} \mathrm{~kg} / \mathrm{kg}$ of brine depending on the assumptions that were made. The total concentration for one of the cases peaked at 1500 years at a value of $10^{-7} \mathrm{~kg} / \mathrm{kg}$ brine. These concentrations resulted in doses to an individual eating beef from cattle watered by the stock well that were well within the International Commission on Radiological Protection guidelines of 100 mrem per year. Cattle might be expected to drink the water, but not humans because the water is almost unpalatable due to the high concentration of dissolved solids.

U.S. DOE (Department of Energy). 1990. Final Supplement Environmental Impact Statement, Waste Isolation Pilot Plant. DOE/EIS-0026-FS. Washington, DC: U.S. Department of Energy, Office of Environmental Restoration and Waste Management. 


\title{
APPENDIX G: TEAM B REPORT: THE DEVELOPMENT OF MARKERS TO DETER INADVERTENT HUMAN INTRUSION INTO THE WASTE ISOLATION PILOT PLANT (WIPP)
}

\author{
Prepared by Team B: \\ Victor R. Baker (University of Arizona) \\ Frank D. Drake (Iick Observatory) \\ Ben R. Finney (University of Hawaii) \\ David B. Givens (American Anthropological Association) \\ Jon Lomberg (Scientific Illustration, Honaunau, Hawaii) \\ Louis E. Narens (University of California-Irvine) \\ Wendell S. Williams (Case Western Reserve University) \\ David B. Givens, B-Team Editor
}

22 April 1992 


\section{APPENDIX G: TEAM B REPORT: THE DEVELOPMENT OF MARKERS TO DETER INADVERTENT HUMAN INTRUSION INTO THE WASTE ISOLATION PILOT PLANT (WIPP)}

FINAL REPORT FOR SANDIA NATIONAL LABORATORIES

$$
\text { -On- }
$$

THE DEVELOPMENT OF MARKERS TO DETER INADVERTENT HUMAN INTRUSION INTO THE WASTE ISOLATION PILOT PLANT (WIPP)

B-Team Report

$$
\text { By }
$$

Victor R. Bakex (University of Arizona)

Frank D. Drake (Lick Observatory)

Ben R. Finney (University of Hawaii)

David B. Givens (American Anthropological Association) Jon Lomberg (Scientific Illustration, Honaunau, Hawaii)

Louis E. Narens (University of California-Irvine)

Wendell s. Williams (Case Western Reserve University)

David B. Givens, B-Team Editor

22 April 1992 
Appendix G: Team B Report

CONTENTS

Preface ................................ G-9

Marker Design Characteristics .......................11

1. General Description ...........................11

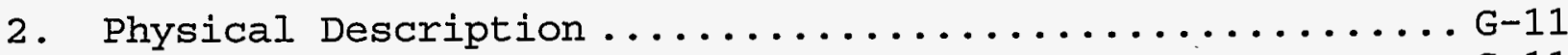

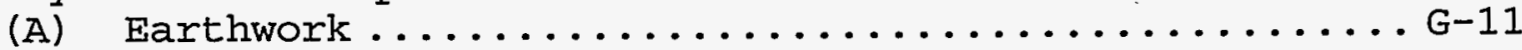

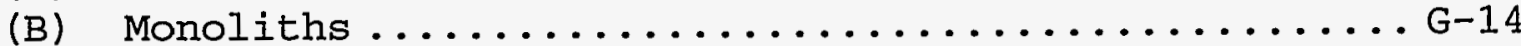

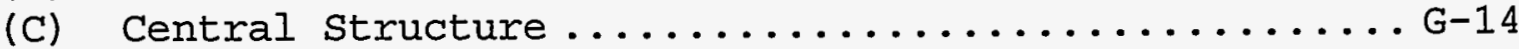

(D) Small, Buried Time Capsules .................. G-14

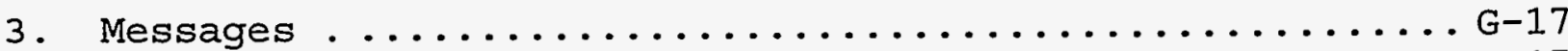

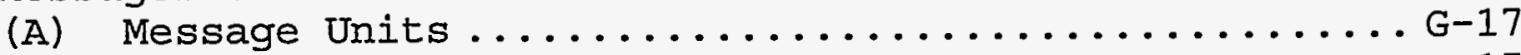

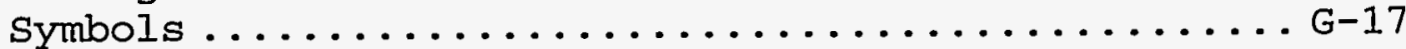

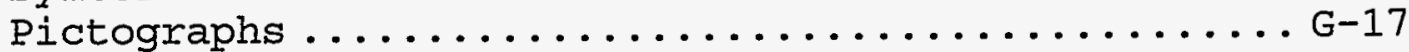

(B) Message Content ......................... -17

Level I: Rudimentary Information ............... G-17

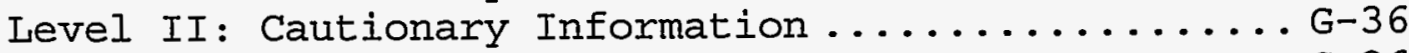

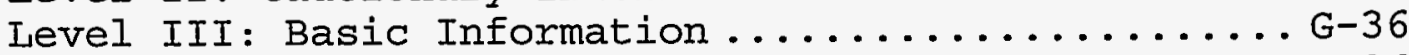

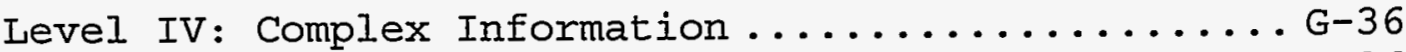

Celestial Reference Points .................... G-36

Individual Marker Performance ....................

4. Persistence ............................. . .

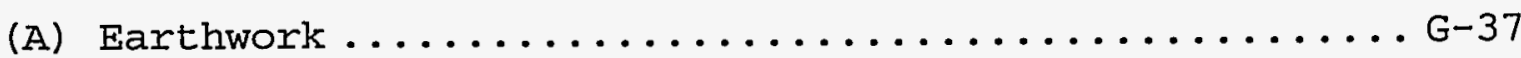

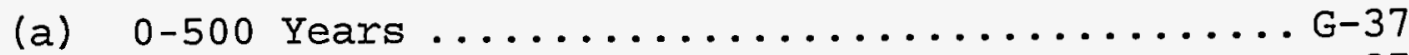

(b) $500-2,000$ Years ......................... G-37

(c) $2,000-10,000$ Years .................... G-37

(B) Monoliths ............................ G-37

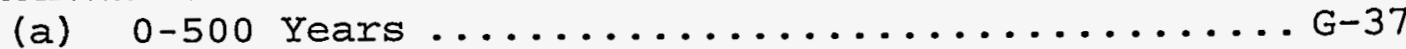

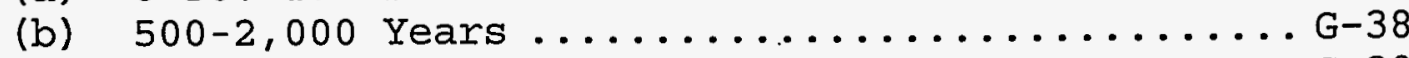

A Representative Granite Monolith...........G-38

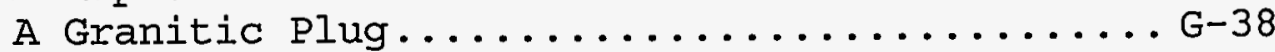

(c) $2,000-10,000$ Years ...................... G-38

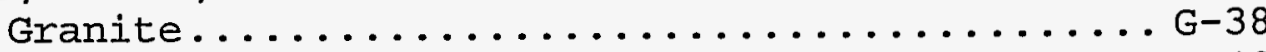

salt Weathering....................... G-40

Sulfuric Acid.......................... 40

sand............................. $4-4$

(C) Central structure .......................

(a) $0-500$ Years ............................... 41

(b) $500-2,000$ Years .............................. 41

(c) $2,000-10,000$ Years ...................... G-41 
(D) Inscriptions (Symbols, Iconic Pictographs, Linguistic Scripts, Narrative Arrangements and Complex Scientific Diagrams) ................ 41

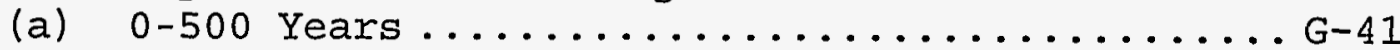

(b) $500-2,000$ Years ........................ -42

(c) $2,000-10,000$ Years .....................G-42

(E) Buried Time Capsules ....................... G-42

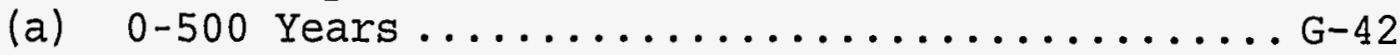

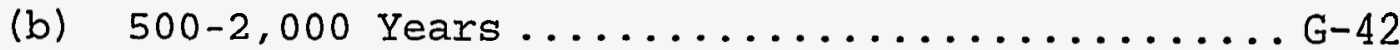

(c) $2,000-10,000$ Years .....................G-42

5. Recognition ............................. G-43

(A) Earthwork ............................ 43

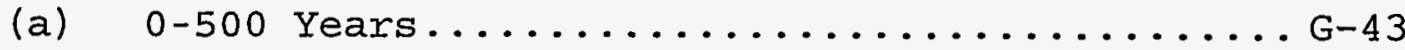

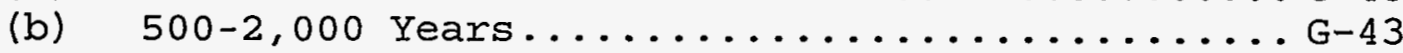

(c) $2,000-10,000$ Years...................... G-43

Marking with High Dielectric Materials ............G-43

(B) Monoliths ..........................

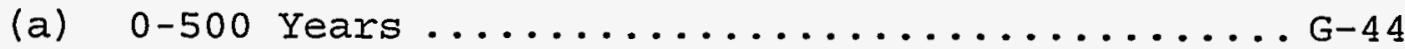

(b) $500-2,000$ Years ........................ G-44

(c) $2,000-10,000$ Years ........................ 45

A Human Dimension .......................... -45

(C) Central structure ....................... 4 . 45

"Made by Humans" ........................... 45

Central Placement ....................... -45

World's Longest-Lasting Human Artifact ........... G-46

(D) Symbols ...........................G-46

International Biohazard symbol ................ G-46

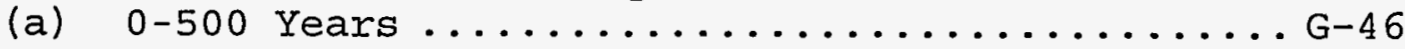

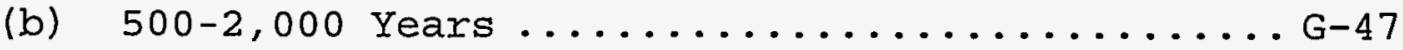

(c) $2,000-10,000$ Years ...................... G-47

(E) Iconic Pictographs ........................

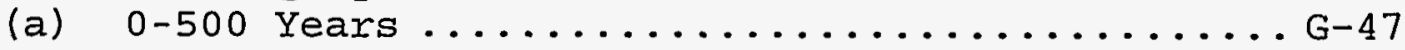

(b) $500-2,000$ Years ........................... 48

(c) $2,000-10,000$ Years ...................... 48

(F) Linguistic scripts ..................... G-48

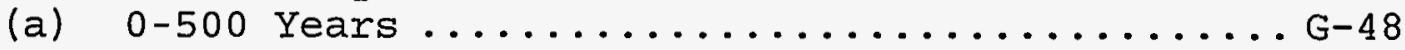

(b) $500-2,000$ Years ......................... 48

(c) $2,000-10,000$ Years ..................... G-48 
Appendix G: Team B Report

(G) Complex Scientific Diagrams ................. G-50

(a) $0-500$ Years ........................... G-50

(b) $500-2,000$ Years ........................ G-50

(c) $2,000-10,000$ Years $\ldots \ldots \ldots \ldots \ldots \ldots \ldots \ldots \ldots$ G-50

(H) Astronomical References ....................

A Millennial Marking System ................ G-51

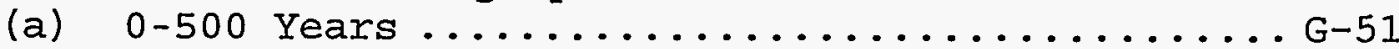

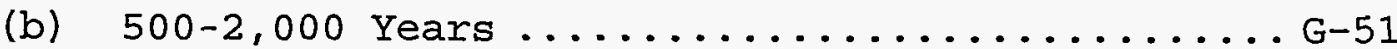

(c) $2,000-10,000$ Years $\ldots \ldots \ldots \ldots \ldots \ldots \ldots \ldots \ldots \ldots$ G-58

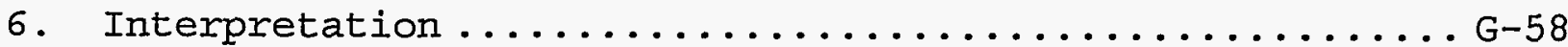

(A) Earthwork ............................. G-58

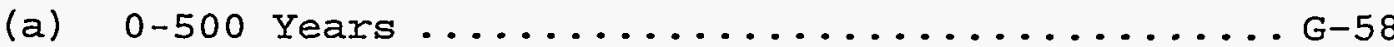

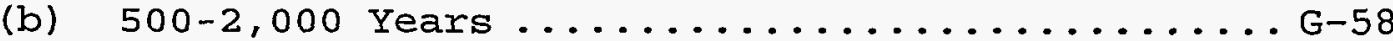

(c) $2,000-10,000$ Years $\ldots \ldots \ldots \ldots \ldots \ldots \ldots \ldots \ldots$ G-59

(B) Monoliths and Central structure ...............5-59

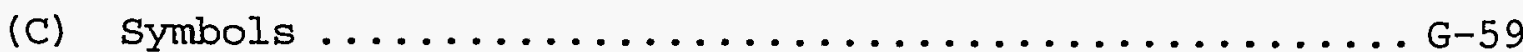

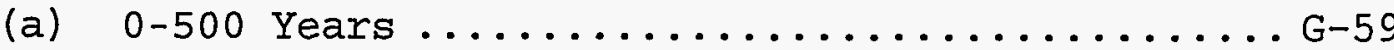

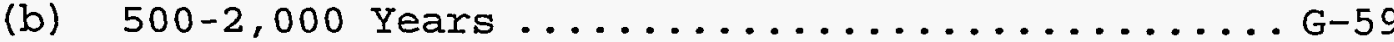

(c) $2,000-10,000$ Years ...................... 59

(D) Iconic Pictographs .....................6-60

(a) $0-500$ years .........................6-60

(b) $500-10,000$ Years .....................6-60

"Consequential" Statement ...................6-60

Historical Sequence ..................... G-60

(E) Linguistic Scripts ..................... G-60

(F) Complex Scientific Diagrams ................G-62

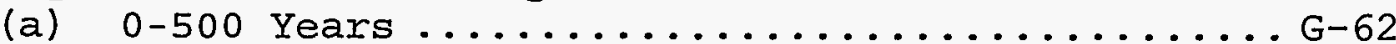

(b) $500-2,000$ Years ......................6-62

(c) $2,000-10,000$ Years ....................6-62

7. Deterrence ............................ $6-62$

(A) Earthwork ........................6.62

(a) $0-500$ Years ........................6-62

(b) $500-10,000$ Years .....................6-63

(B) Monoliths and Central Structure ..............G-63

(a) $0-500$ Years .........................6-63

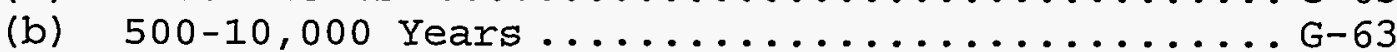

(C) Symbols ..........................6-63

Marker System's Performance ....................6-64 
8. Recognition of Marker system .................6-65

More Advanced, As Advanced, and Less Advanced Societies .G-65 Political Change .......................... G-65 Mescalero Apache Symbolism ...................G-66 Vandals ..............................G-66 Radical Increase in Consumption of World Resources ...... G-69 Radical Discontinuity ...................... G-69 References Cited ............................. Appendix A: Testing Marker Systems for Understandability ... G-73 Appendix B: Supplemental Material on the WIPP Marker...... G-76

\section{Figures}

1. Radiation Trefoil Used for Earthworks, at Closure and After 5000 years .......................... G-12

2. Skull and Crossbones Used for Earthworks, at Closure

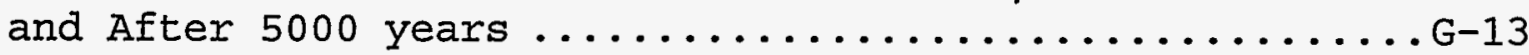

3. Tall Granite Monolith with Inscriptions .............15

4. Cross-Sectional Model of the WIPP and the Underground ...G-16

5. Pictographic Definition of Symbols-Circle with Slash....G-18

6. Pictographic Definition of Symbols-Skull and Crossbones ...............................

7. Pictographic Definition of Symbols-Radiation Trefoil....G-20

8. Defining the Equality of Symbols, Version $1 \ldots \ldots \ldots \ldots$.

9. Defining the Equality of Symbols, Version $2 \ldots \ldots \ldots \ldots$.

10. Defining the Equality of Symbols and Message Languages,

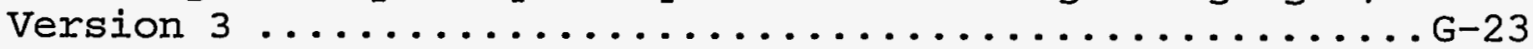

11. Defining the Equality of Symbols, Version $4 \ldots \ldots \ldots \ldots$. 12. Defining the Equality of Symbols, Version $5 \ldots \ldots \ldots \ldots$. 25 13. Pictographs Showing the Passage of Time at the WIPP ....G-26 14. Egyptian Funerary Art ..................... 49 
15. The Duhrva-Darshak Yantra, or Pole-Star Instrument .....G-52

16. Millennial Marking system .....................

17. The Changing Shape of the Big Dipper Over 100,000 Years ................................ 61

18. The Quartered Circle: Visual Representations of the Mescalero Apache "Base Metaphor" .................G-67

19. Transformations of the Mescalero Apache Quartered Circle into a Four-Pointed Star, then Mountains ........G-68

20. Transparent Canister of Sample WIPP waste ............6-83

21. View of a $30^{\prime}$ Monolith from the WIPP Buildings ........G-87 
Preface

After an informal discussion as the nascent "B-Team," on the last day of the Marker Panels meeting hosted by Sandia National Laboratories from November 4-6, 1991 in Albuquerque, New Mexico, five members of the group (Drake, Finney, Givens, Lomberg and Narens) met formally on the weekend of December 14-15, 1991 in Kona, Hawail. Three (Givens, Lomberg and Narens) met on December 16, 1991 to discuss testing the markers. Before the Kona meeting, B-Team members had been asked by Jon Lomberg (Chair) to respond to questions designed to assess overall agreement/disagreement on the issues.

The five team members who met in Hawaii found themselves in general agreement on most of the issues. Wendell Williams' comments, faxed to Hawaii, seemed in accord. Victor Baker's input, unfortunately, was not available for comment.

There was unanimous agreement on the following points:

(1) The site should be marked, on the assumption that leaving it unmarked would pose greater risks to the future. Current mining activities in the area, alone, would make the choice of not marking extremely risky for present-day (i.e., living) humans, and cumulatively more dangerous for those living between now and 12,000 A.D. At present the WIPP is in an area of active oil production, gas production and potash mining (Pasztor, 1991, IX-27).

(2) Surface and buried markers should be used in tandem to enhance message redundancy.

(3) All message components should be truthful, consistent and noncontradictory.

(4) Only the land directly above the waste panels themselves -about a $1 / 2$ square-kilometer area-should be marked. (A) This would put the marker system on a cognitive scale better geared to human perception than one spread thinly over 16 square miles. (B) Additionally, it would reduce confusion that could arise from boring beneath a marker system beyond the panels and uncovering nothing unusual.

(5) The entire perimeter of the marker system should be visible from the center of the site.

(6) An assortment of symbolic, pictographic, linguistic, narrative, diagrammatic, scientific and astronomic messages should be used to ensure that people from any conceivable 
culture or future society would be able to understand that hazardous materials are buried in the immediate area and that they should not intrude.

(7) Part of the WIPP building itself should be left as evidence for future archeologists.

(8) Information about the WIPP should be archived off-site, but details should be left to more knowledgeable archivists and library-science specialists 50 years from now.

(9) Marking for nuclear waste sites should be standardized worldwide. Each site should include as part of its marking system a map of all other nuclear waste sites in the world.

(10) Regarding the markers and their messages, whatever can be tested, should be tested.

David B. Givens

B-Team Editor

Washington, DC 


\section{Marker Design Characteristics}

\section{General Description}

We recommend that the proposed marker system consist of the following components:

(A) Berms or earthworks to help define the perimeter of the surface area directly above the waste repository. The earthwork might be arranged in the shape of a symbol, yet to be determined.

(B) A ring of granite monoliths, around or within the perimeter of the marked area, bearing a variety of symbolic, pictographic and linguistic inscriptions.

(C) A central granite structure to house more detailed textual, narrative, diagrammatic and scientific information.

(D) A large number of small, durable markers inscribed with basic warning information, seeded at various depths within the marked area and in the surrounding earthworks.

(E) Buried duplicates of the granite monoliths placed in key locations at various depths, such as in the plugs of sealed airshafts.

(F) A layer of contrasting dielectric materials at the surface to permit remote detection by radar (perhaps arranged in the shape of the designated marking symbol).

(G) Duplicates of markers placed in Carlsbad Caverns and in off-site archives.

\section{Physical Description}

(A) Earthwork. A 30' high earthwork, built of local sedimentary materials and caliche, could be constructed in a geometric shape, perhaps in the shape of a designated warning symbol. The earthwork would surround the $1 / 2$ square-kilometer area above the waste panels, and could enclose an inner ring of monoliths. By imbedding a thin layer of non-local sediments with different dielectric, radar reflective and magnetic properties, the earthwork could be remotely sensed by aircraft and orbital satellites. (Figures 1 and 2 exemplify how the marking system might appear. The top image in each figure shows the site soon after completion, and the bottom image depicts the site after many centuries of degradation; the actual choice of symbols needs further study. [The structure just outside the ring of monoliths in each figure is the existing WIPP facility.]) 


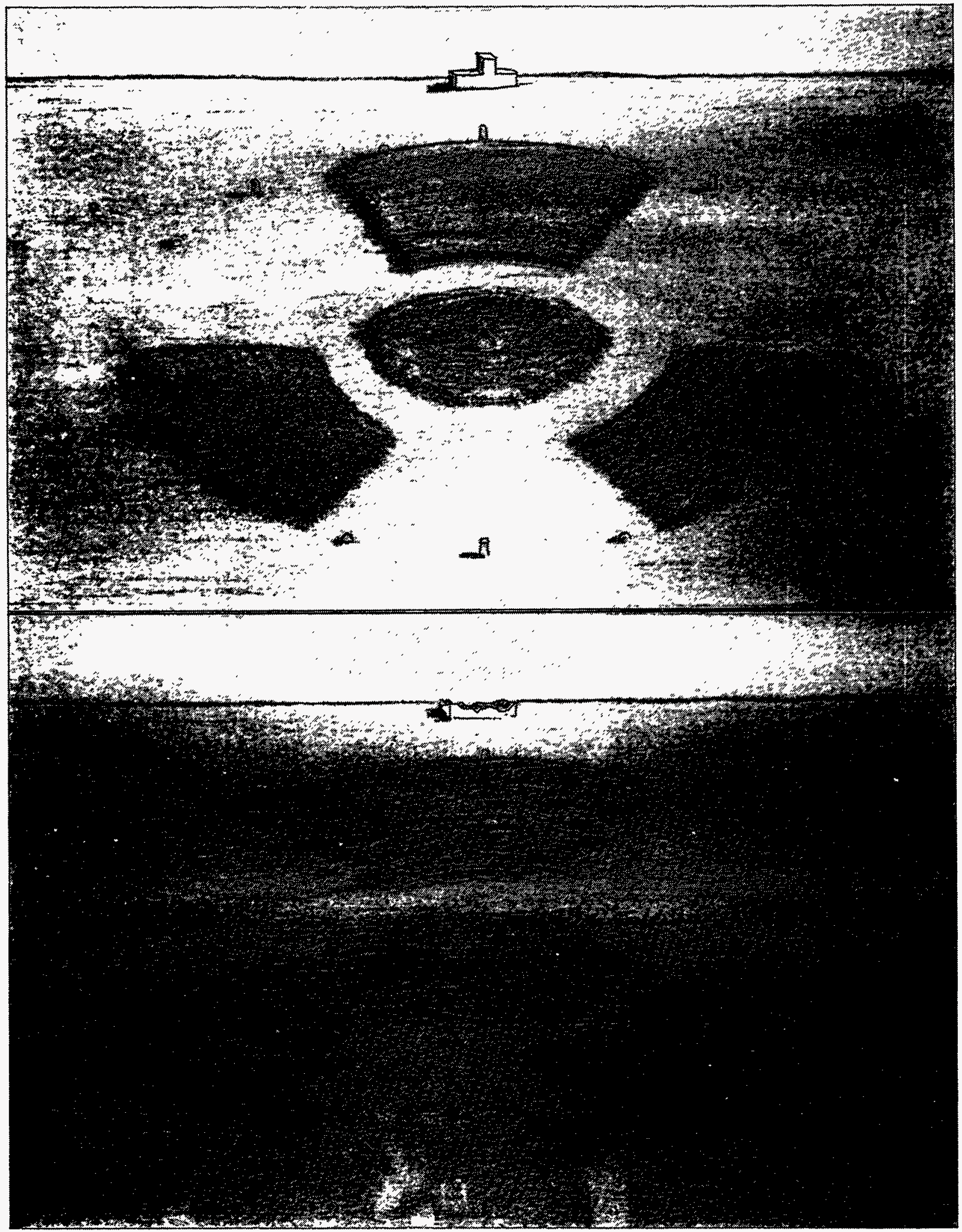

Figure 1. Radiation Trefoil Used for Earthworks, at Closure and After 5000 Years (art by Jon Lomberg). 


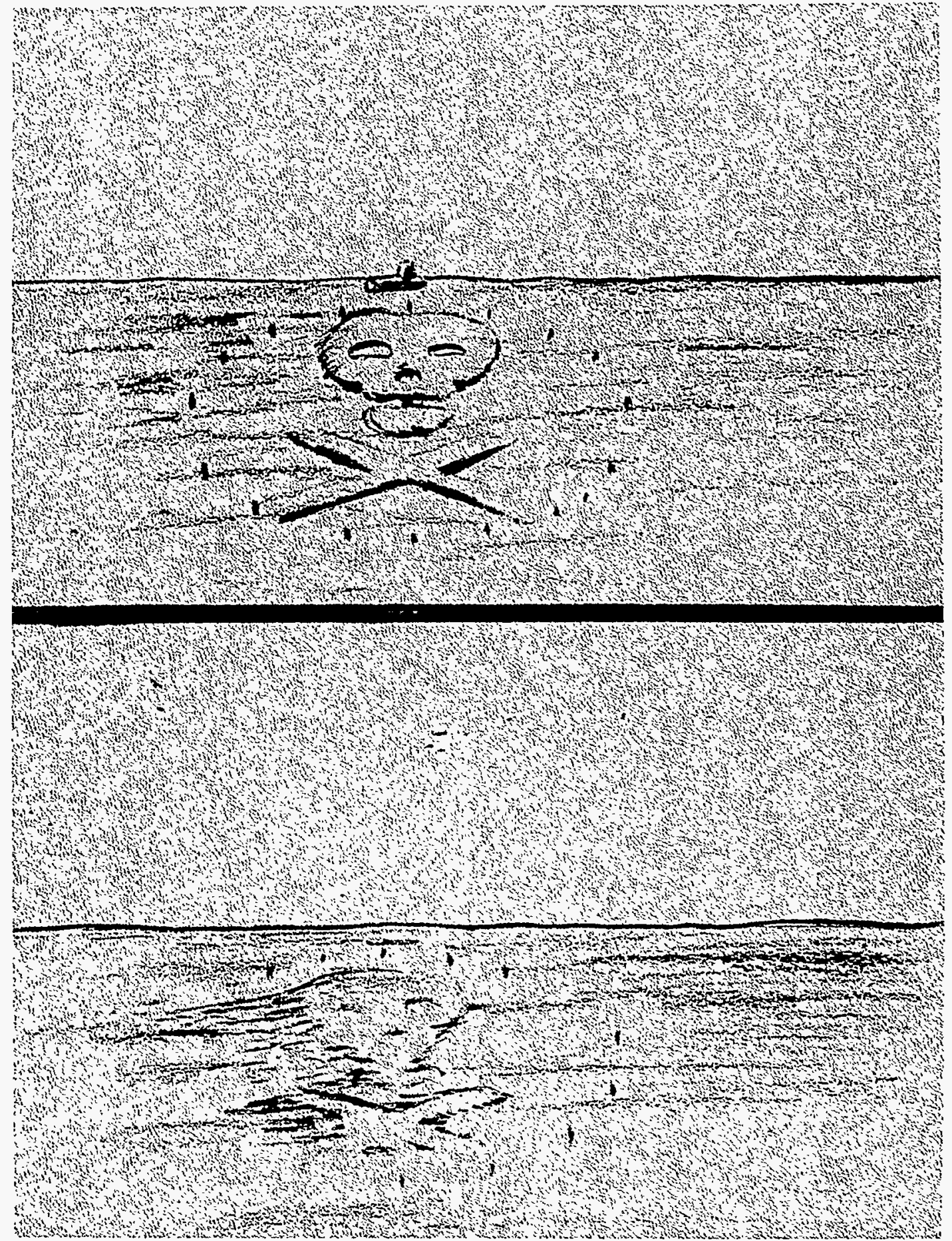

Figure 2. Skull and Crossbones Used for Earthworks, at Closure and After 5000 Years (art by Jon Lomberg). 
(B) Monoliths. Free-standing, massive, one-piece granite monoliths could be placed in a circular arrangement within the earthwork. Visible, tall monoliths (25' high $X 10^{\prime}$ wide) and stable, short, rounded stones (10' high $\mathrm{X} 20^{\prime}$ wide) would alternate in the arrangement. The tall monoliths would be designed to be visible despite the encroachment of sand. The squat monoliths would be designed to be difficult to topple or decapitate. Both types of monoliths (as well as additional, buried monoliths and a large, granitic plug in the main mineshaft off-site) would be worked and shaped to convey they were manufactured by humans. Each monolith would carry a variety of inscriptions. The inscriptions would be placed on protected surfaces of the monoliths, such as within recessed niches and overhangs (see Figure 3 for a sample design of a tall monolith). The number of monoliths would be a power of two, preferably 16 or 32 , to help future investigators infer the original configuration of the ring, should some elements be missing.

(C) Central structure. A granite "rock shelter" (20' high X 30' base) could be constructed inside the monolithic ring. Extensive planar stone surfaces within the structure (protected from weathering) would carry linguistic, diagrammatic and pictographic inscriptions. It should be designed to discourage habitation and vandalism. The kind of information that would be inscribed in the central structure would include a map of all nuclear waste sites worldwide; detailed schematics of the repository and its contents; a diagram of the periodic table of the elements with radioactive elements highlighted; and an explanation of how the Earth's processional cycle is to be used in dating the age of the repository.

(D) Small, Buried Time Capsules. A variety of smaller "time capsules" could be buried (beneath the souvenir-hunter's casual digging zone) to deter serious excavators. Candidate materials for the small markers might include baked clay or other ceramics, tektite-like glass or sintered alumina. Durable tablets carrying simpler messages could likely be decoded by less developed societies in the future, and decoded and chronologically dated by as-advanced and more-advanced societies having such analytic tools as thermoluminescence. Buried samples of wood could be dated by carbon-14 analysis. Cross-sectional models using samples of sand, siltstone, gypsum and rock salt to show a cutaway view of the geological strata, mine shaft and waste panels (Figure 4) could be emplaced on and off the WIPP site. 


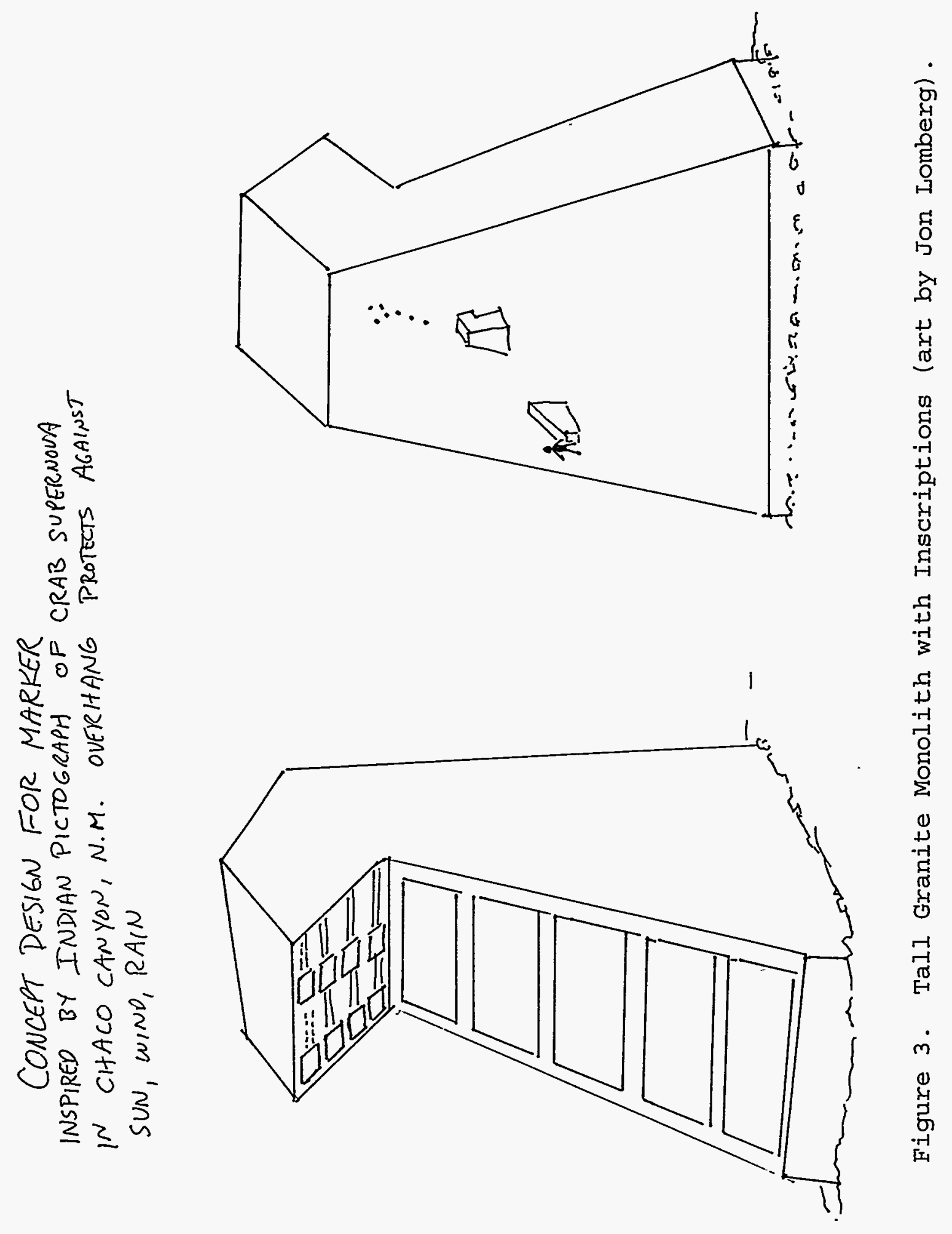




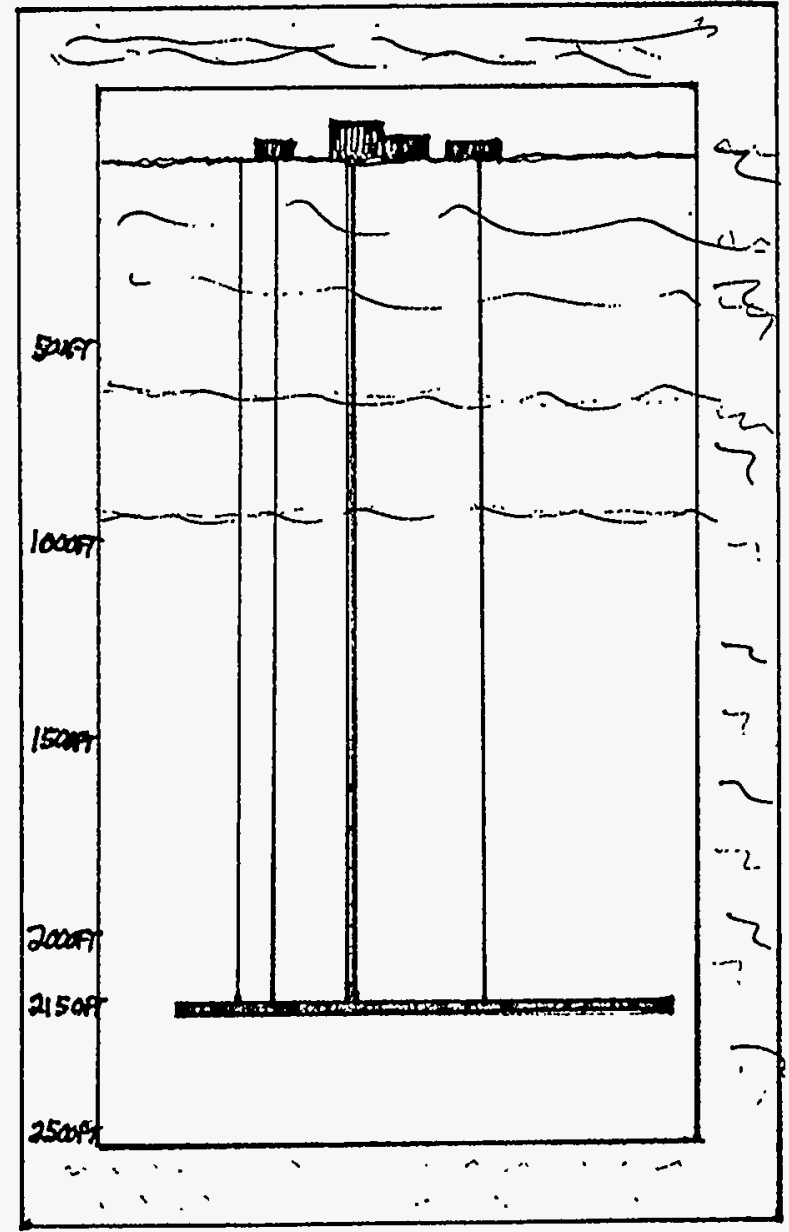

CROSS-SECTIONAL MODEL OF WIPP SITE

Figure 4. Cross-Sectional Model of the WIPP and the Underground. 


\section{Messages}

(A) Message Units. In semiotics, the most generic and fundamental message component is known as a sign. Signs marking the WIPP site would consist of (1) the arranged pattern of the monument itself, its geometric earthwork and configuration of surface and subsurface stones; (2) conventional symbolic shapes, such as the trefoil; (3) iconic pictographs (signs that picture, e.g., the circular arrangement of monoliths or the human face and body); (4) linguistic scripts (of the world's major written languages, including English, Spanish, German, Russian, Japanese and Chinese; major liturgical languages, such as Latin, Hebrew and Arabic; and languages of the region's indigenous people, including Navajo, Hopi and Mescalero Apache); (5) narrative arrangements (sequences of signs that tell a story or explain a consequence of actions); and (6) complex scientific diagrams and notation systems, such as the periodic table of the elements.

Symbols. We recommend that a symbol or a variety of symbols be used. Symbols may have more emotional connotations than other signs. Indeed, symbols such as the U.S. flag, star of David, Christian cross and Nazi swastika can be highly charged to humans. In our discussions, the choice of a nuclear warning symbol itself became somewhat emotional. In particular, some team members felt that the trefoil should be included in the report as an example of a nuclear symbol. Others felt that using the trefoil as an example would prejudice readers, and that the final choice of a designated warning symbol (or symbols) should be left to future researchers. Any symbols used should be defined pictographically so they could be understood by people who had no previous knowledge of the symbols. (See Figures 5-12)

Pictographs. The human being, drawn as a stylized stick figure, ought to be easily recognized by any other human. The existence of a worldwide, pancultural tradition of stick-figure iconography was outlined by one of the team members in a previous report (Givens, 1982). A series of drawings showing stick figures engaged in various activities can, through iconographic principles of narration, show the history of the WIPP as well as the consequences of intrusion. Other drawings can define symbols and show how the markers are to be decoded. (see Figure 13, Parts (a) through (j))

(B) Message Content. The proposed marker system would encode four successive levels of meaning (Givens, 1982), from rudimentary (information low) to complex (information high):

Level I: Rudimentary Information. The site itself and its component parts would announce "something 
Appendix G: Team B Report
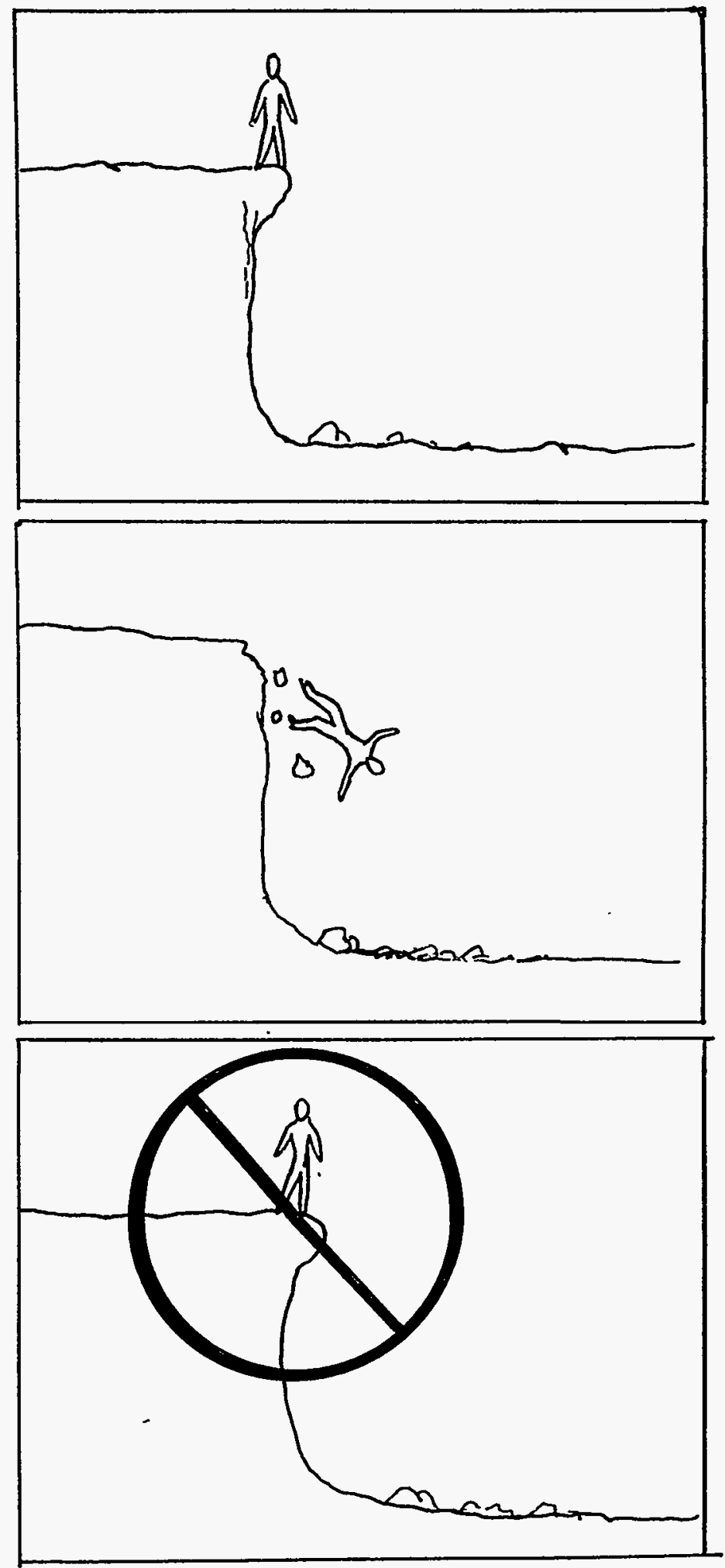

Figure 5. Pictograph Definition of Symbols-Circle with Slash (art by Jon Lomberg). 

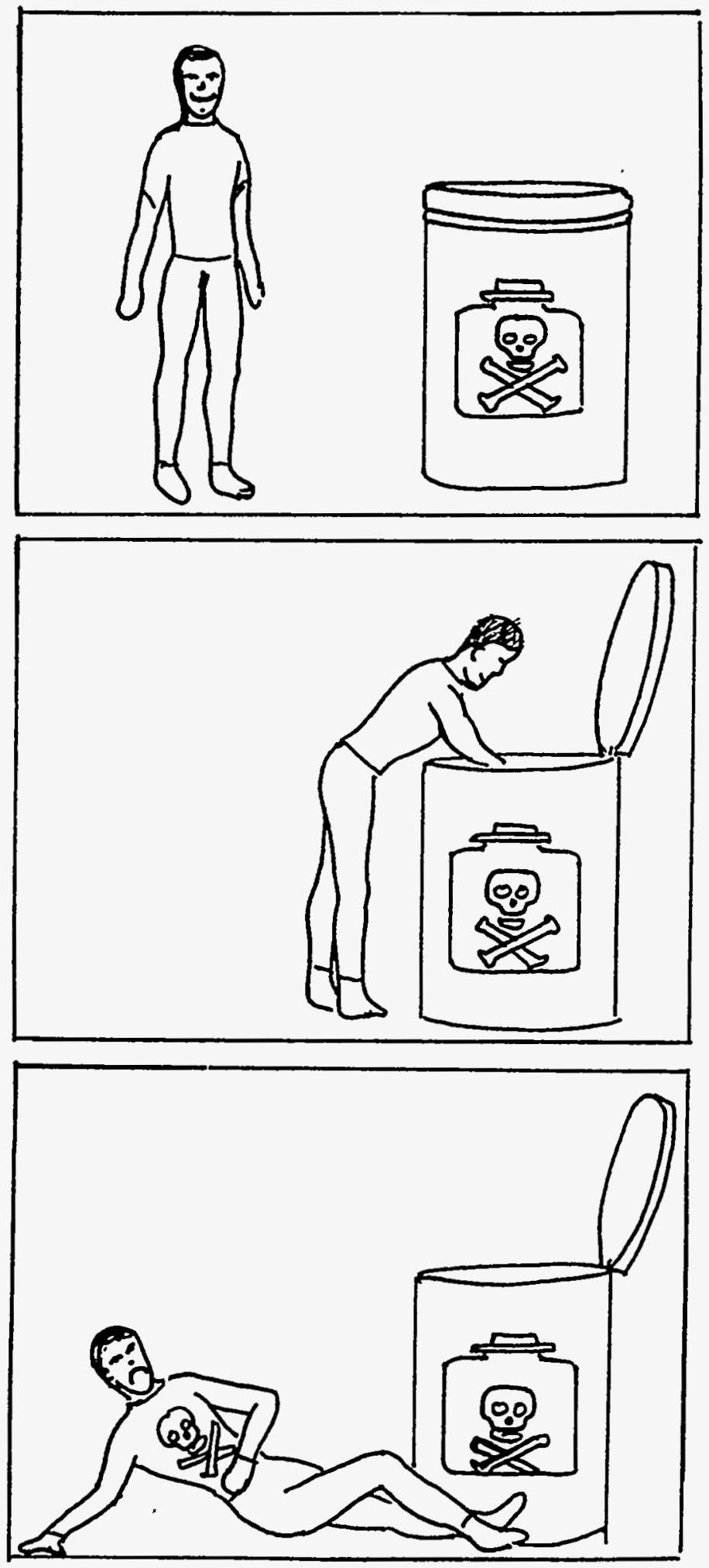

Figure 6. Pictograph Definition of Symbols-Skull and. Crossbones (art by Jon Lomberg). 
Appendix G: Team B Report
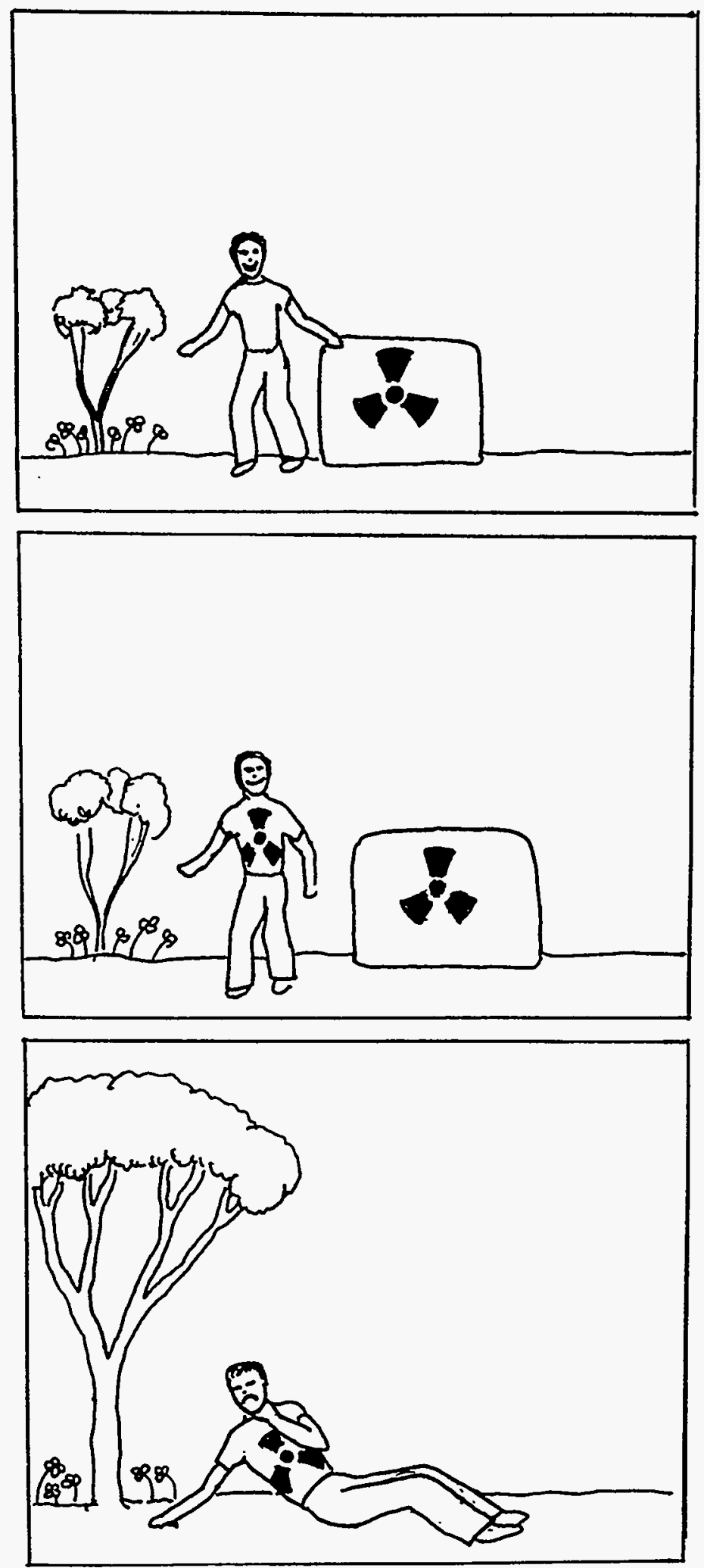

Figure 7. Pictographic Definition of Symbols-Radiation Trefoil (art by Jon Lomberg). 

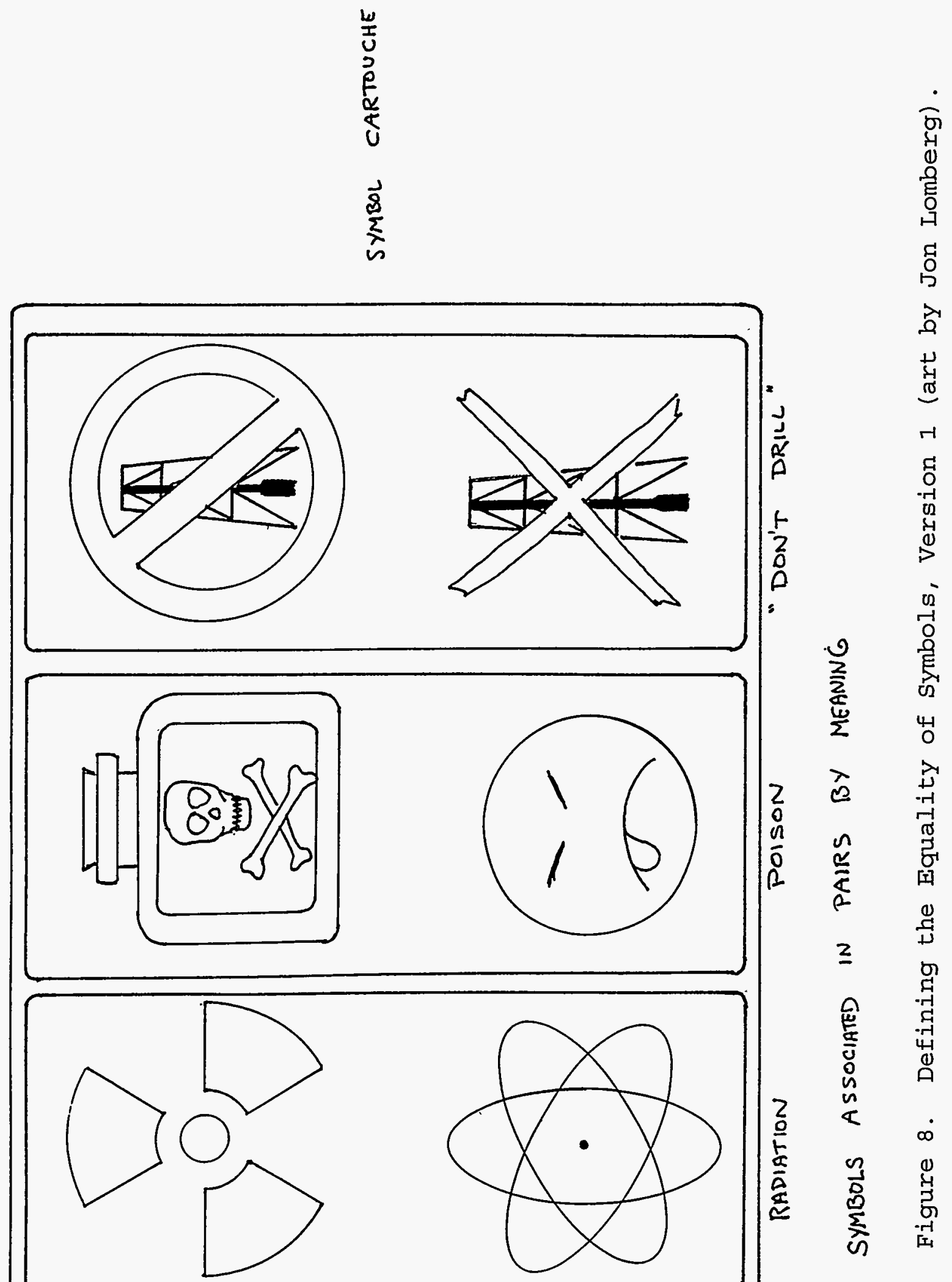


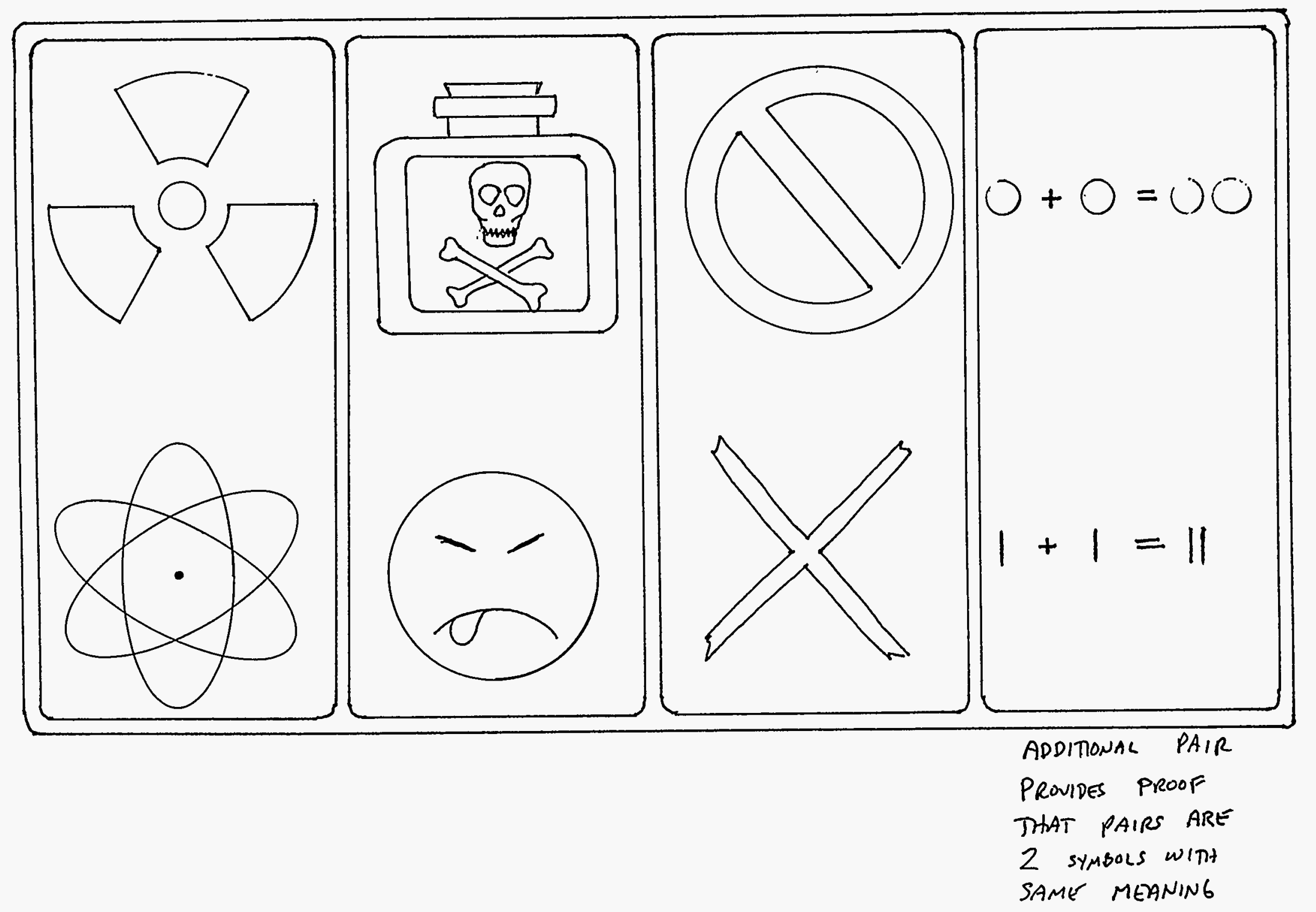

Figure 9. Defining the Equality of Symbols, Version 2 (art by Jon Lomberg). 


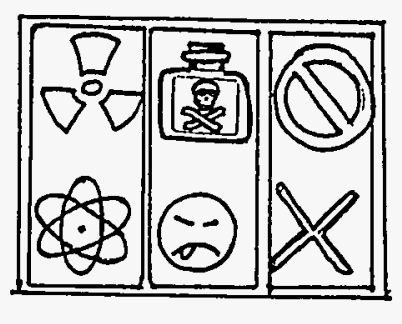

WARNING: radioactive Waste buried here. Poison! No Drilling, NO MINING, NO EXPLOSIVES!

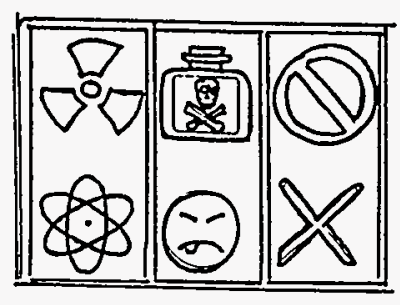
אזהרה : פסולת הדיומקטיבית קבורה פה תעל

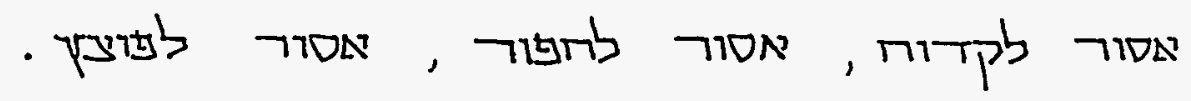
\begin{tabular}{|l|l|l|}
\hline 0 & 0 \\
\hline 0 & 0 & 0 \\
\hline
\end{tabular} CAUTIÓN: Radioactivo subterranea aqul. PoIsón! no trabajo, no explosivos!

USE OF CARTOUCHE WITH LANGUAGES gives a clue that all languages carry tTE same message.

Figure 10. Defining the Equality of Symbols and Message Languages, Version 3 (art by Jon Lomberg). 


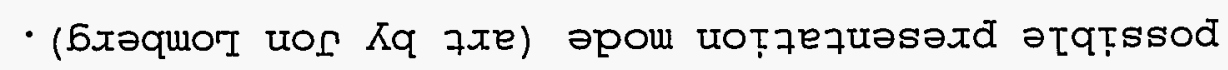

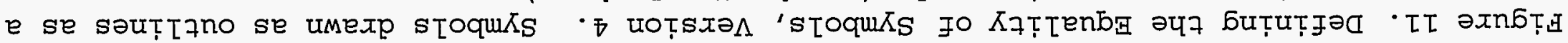
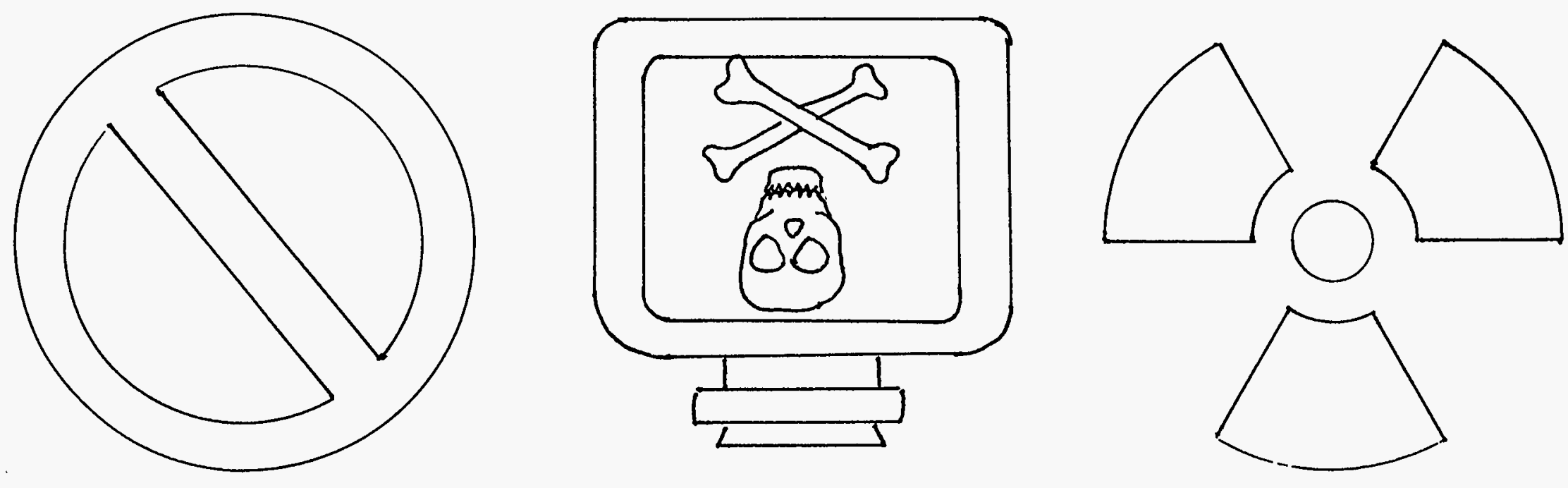

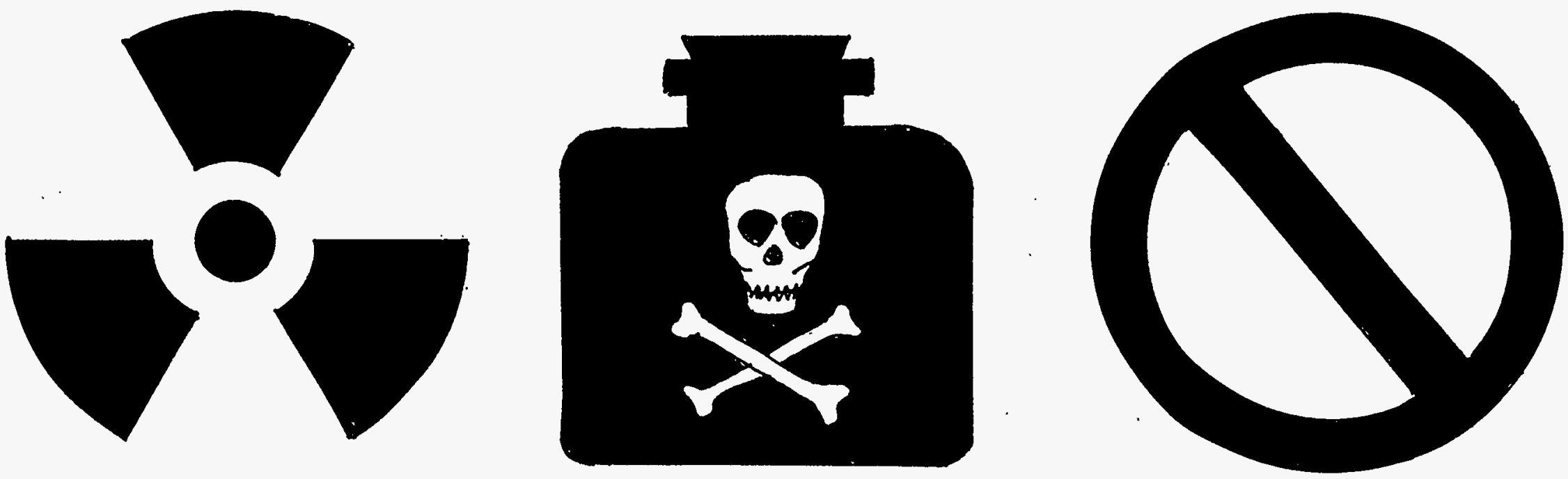

Figure 12. Defining the Equality of Symbols, Version 5. Symbols drawn as filled figures as a possible presentation mode (art by Jon Lomberg). 


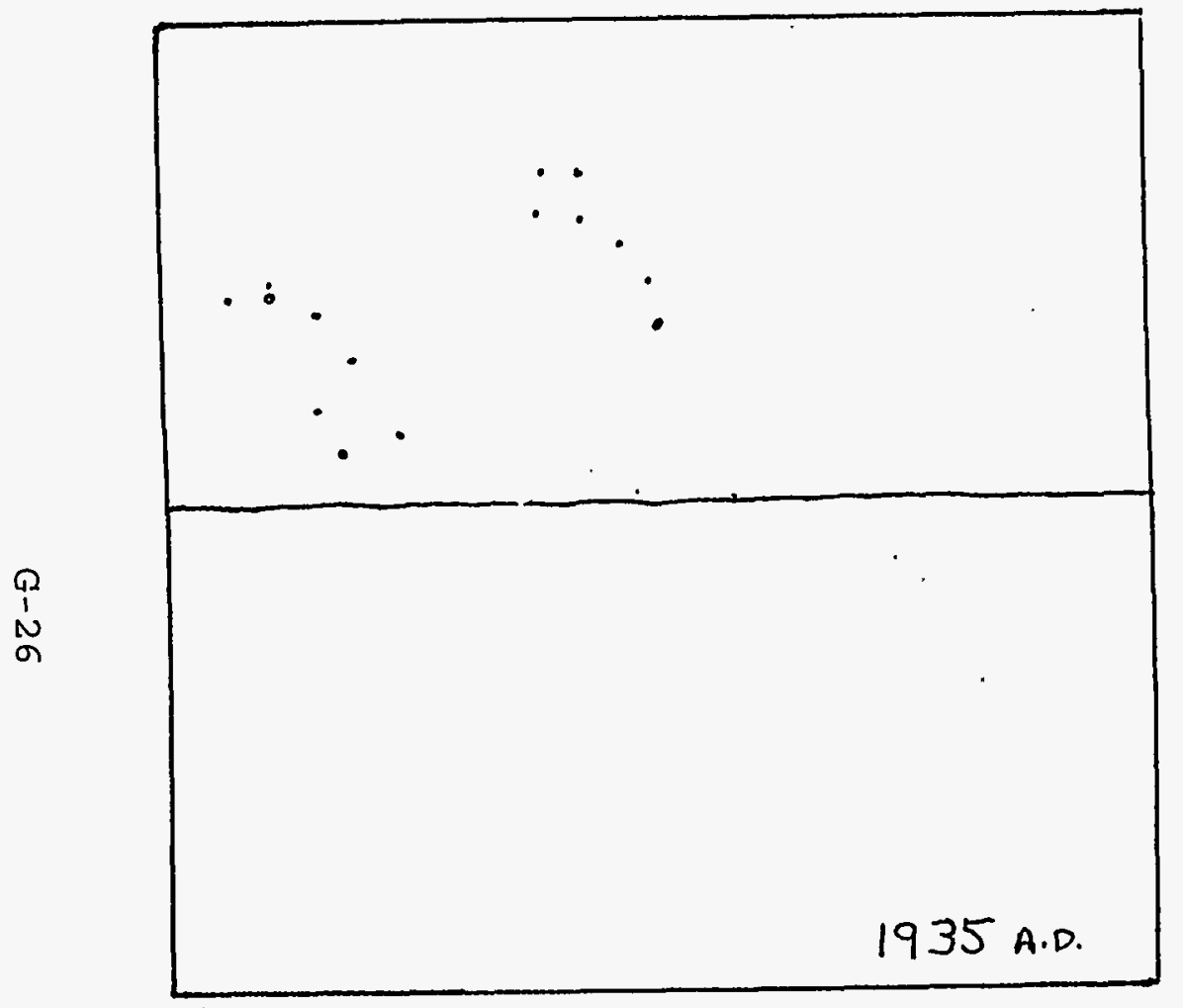

0

Figure 13. Pictographs Showing the Passage of Time at the WIPP. Parts (a) through (j) relate the passage of time with the number of dots, and present a closeup surface picture by a long-range surface and underground picture (art by John Lomberg). (Figure continued on next page.) 


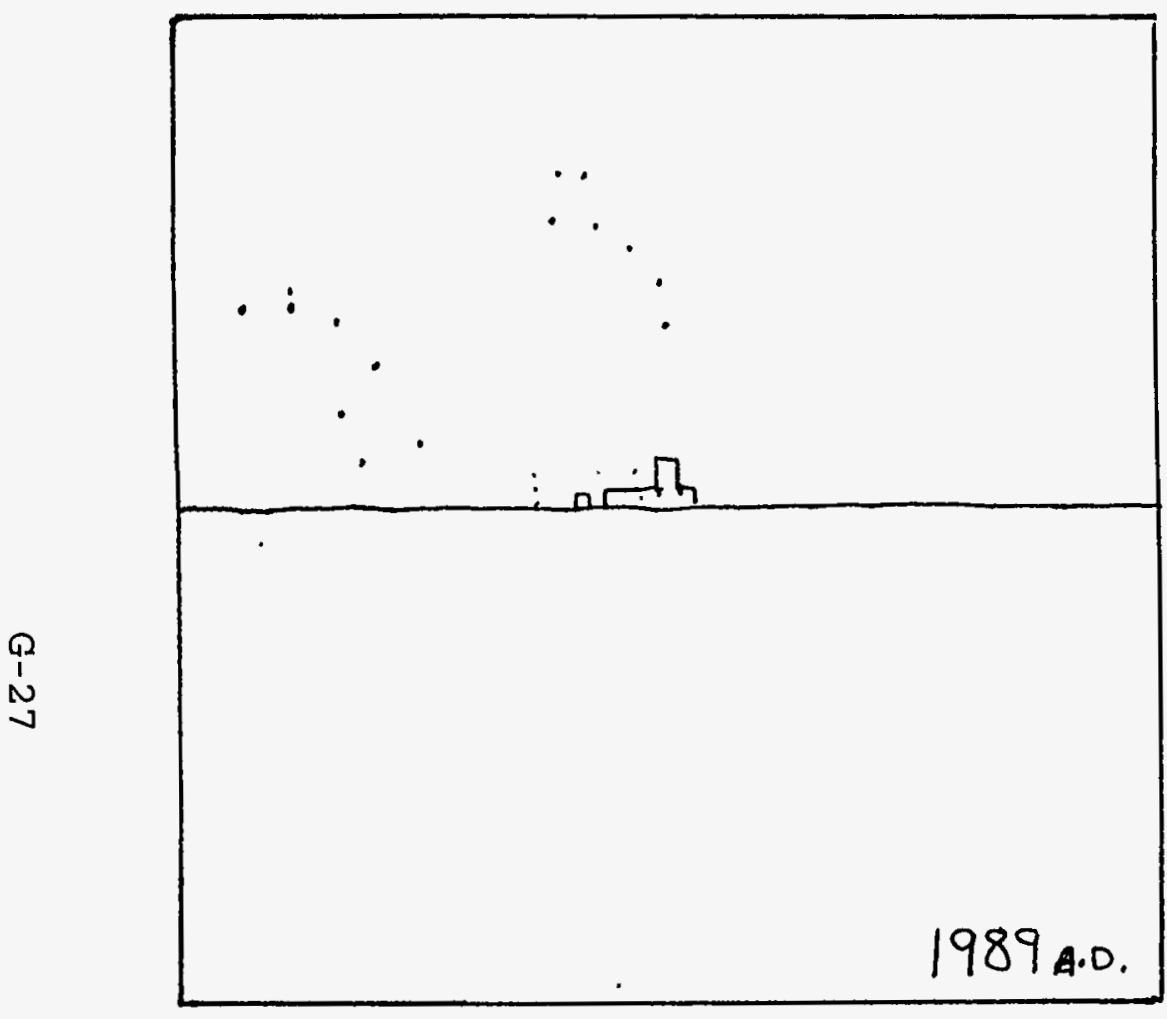

0

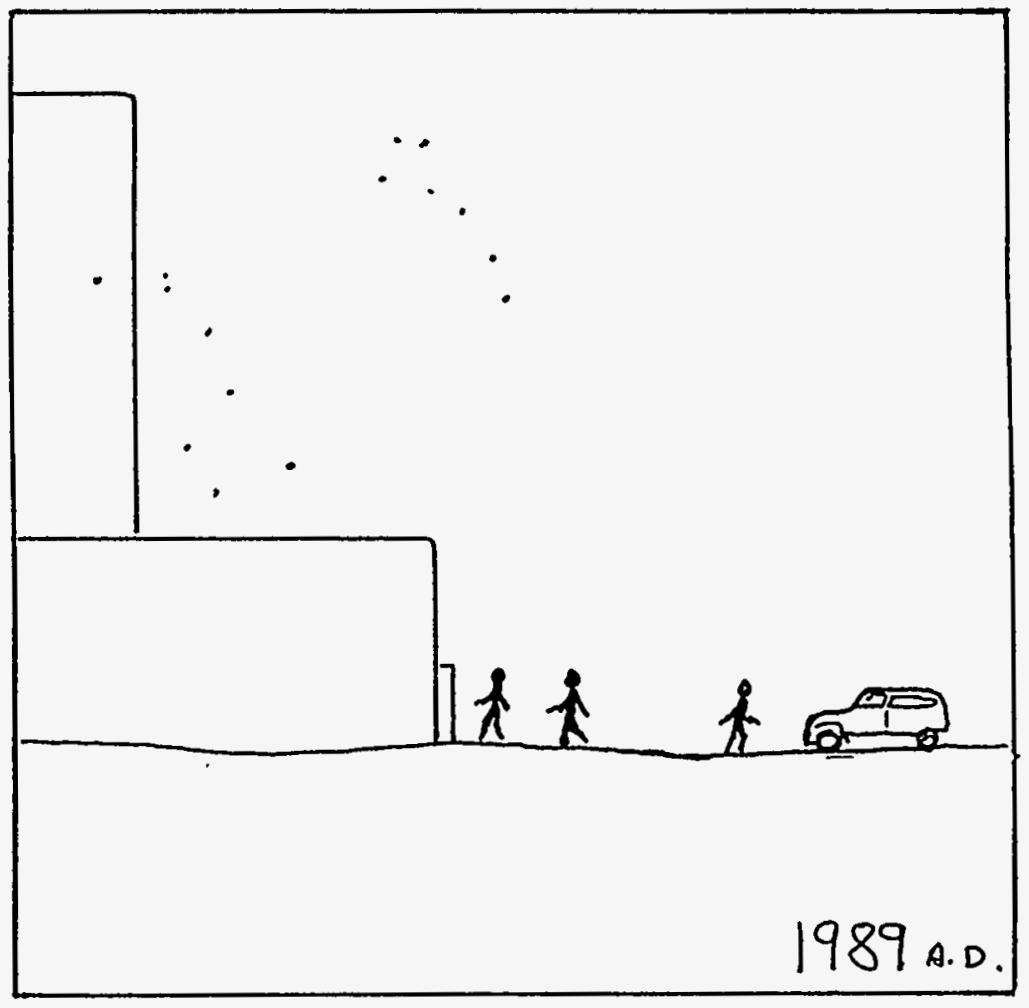

(b)

Figure 13. Pictographs showing the Passage of Time at the WIPP. Parts (a) through (j) relate the passage of time with the number of dots, and present a closeup surface picture by a long-range surface and underground picture (art by John Lomberg). (Figure continued on next page.) 


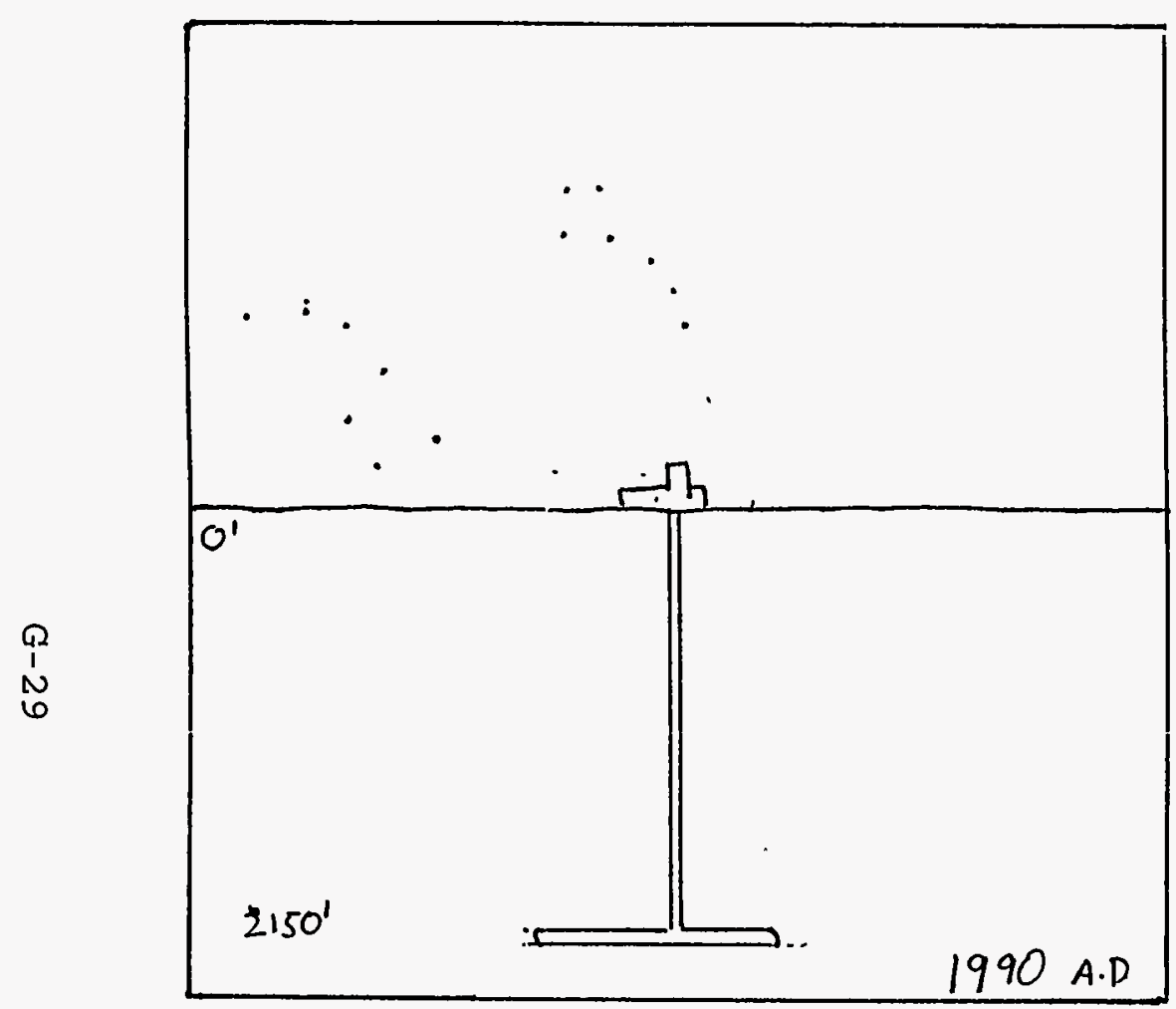

$\bullet \bullet \bullet \bullet$

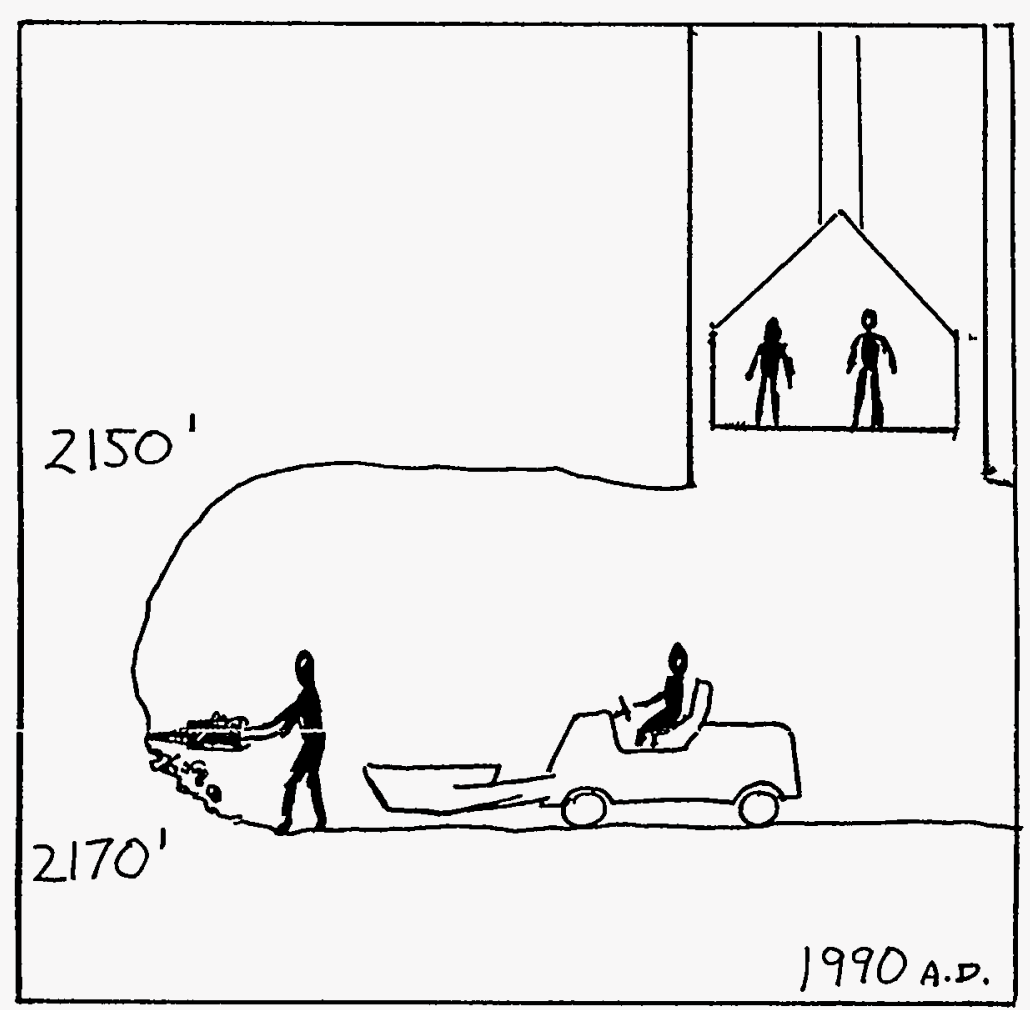

(d)

Figure 13. Pictographs Showing the Passage of Time at the WIPP. Parts (a) through (j) relate the passage of time with the number of dots, and present a closeup surface picture by a long-range surface and underground picture (art by John Lomberg). (Figure continued on next page.) 


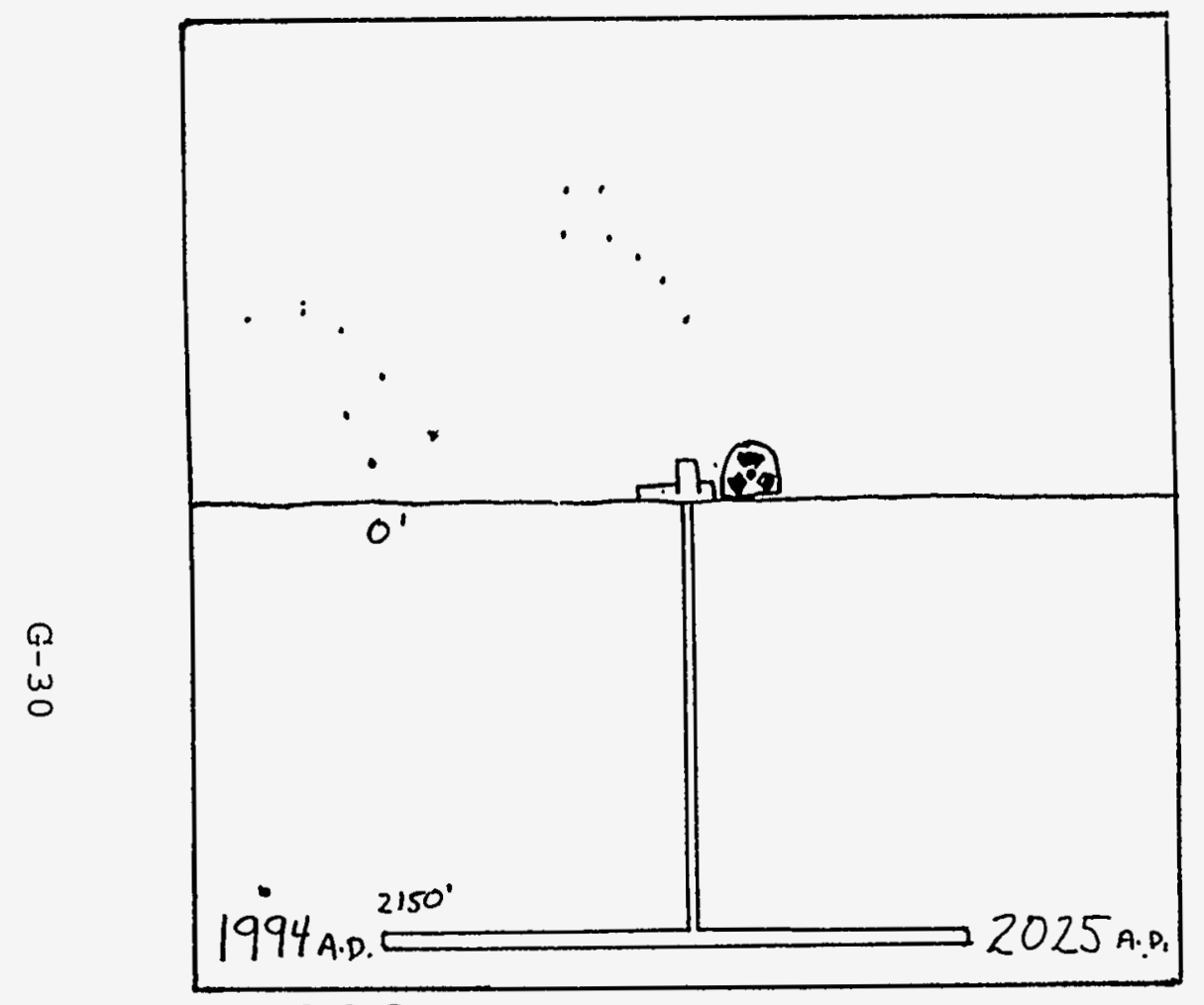

00000

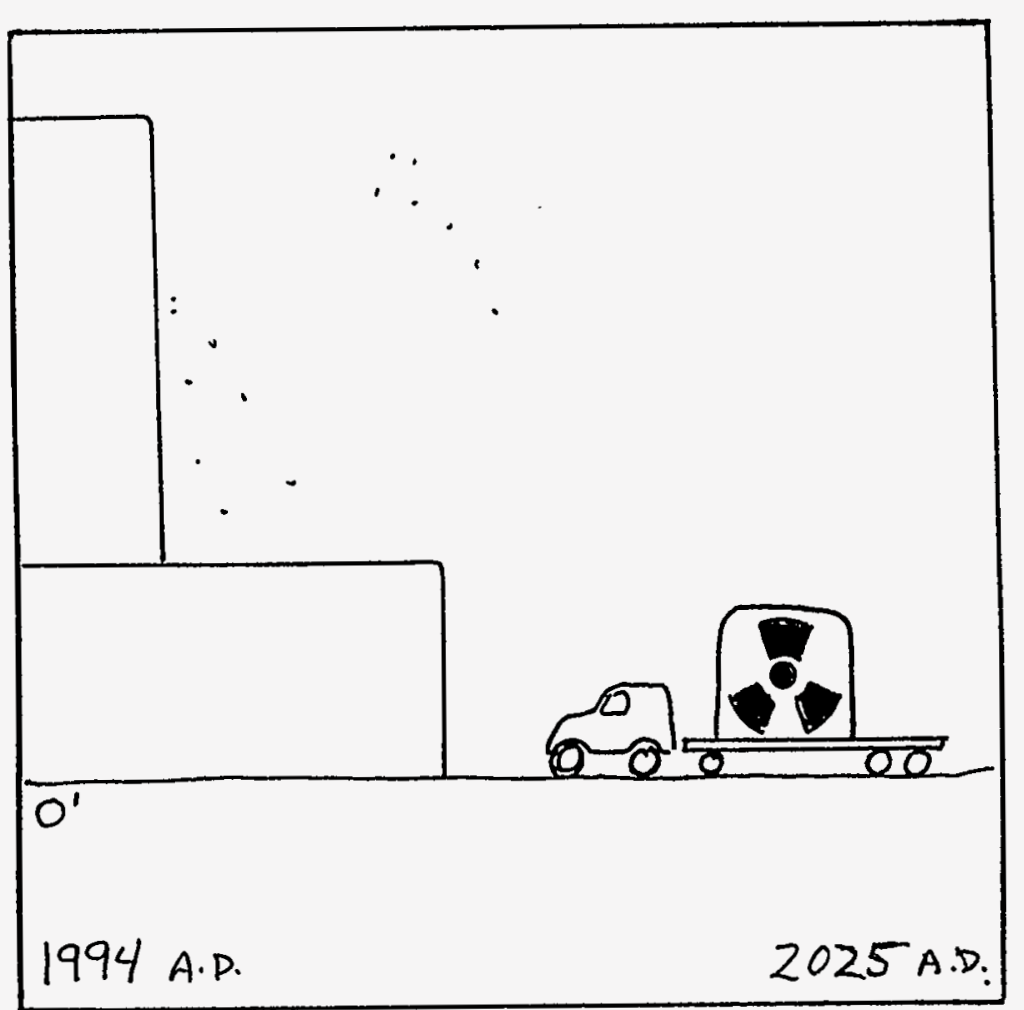

(e)

Figure 13. Pictographs showing the Passage of Time at the WIPP. Parts (a) through $(j)$ relate the passage of time with the number of dots, and present a closeup surface picture by a long-range surface and underground picture (art by John Lomberg). (Figure continued on next page). 


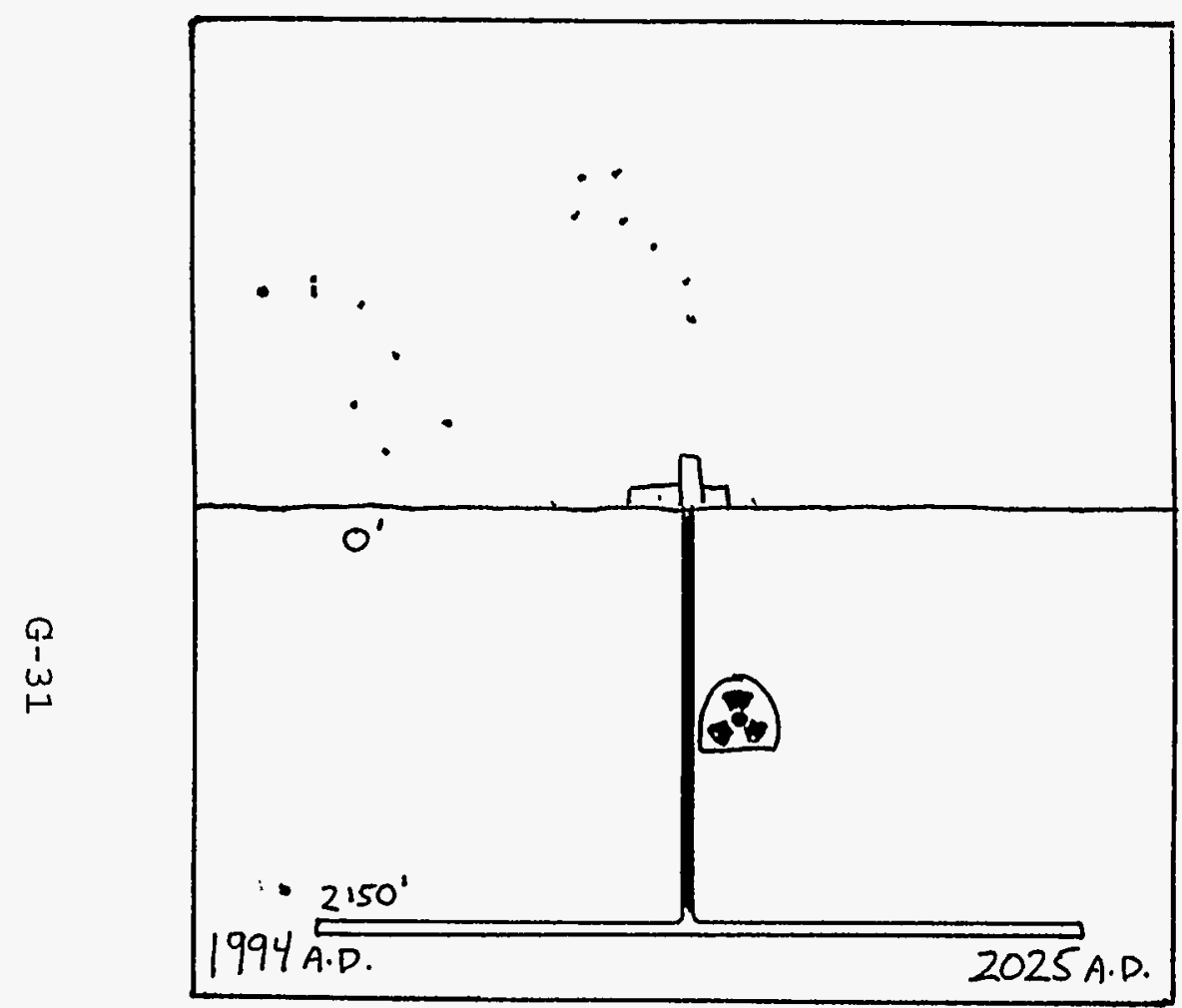

000000

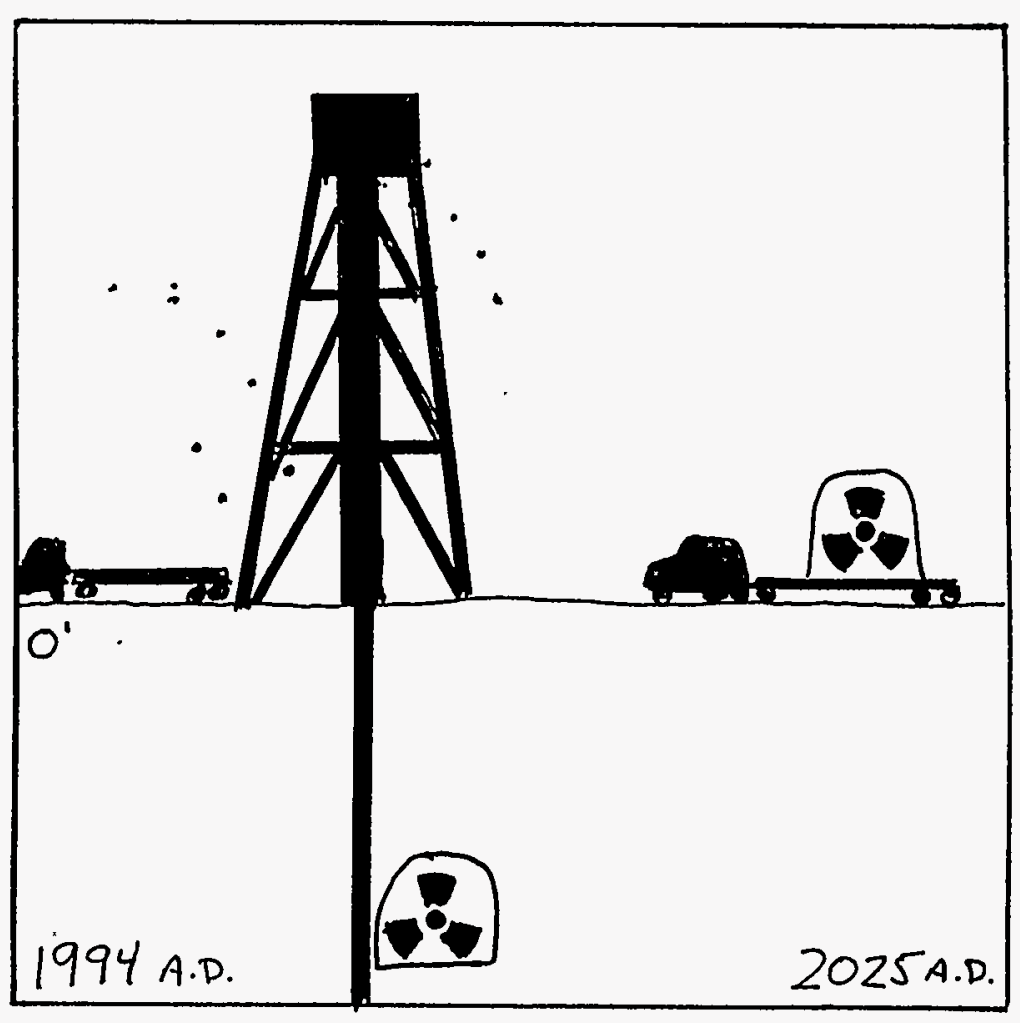

(f)

Figure 13. Pictographs Showing the Passage of Time at the WIPP. Parts (a) through (j) relate the passage of time with the number of dots, and present a closeup surface picture by a long-range surface and underground picture (art by John Lomberg). (Figure continued on next page). 


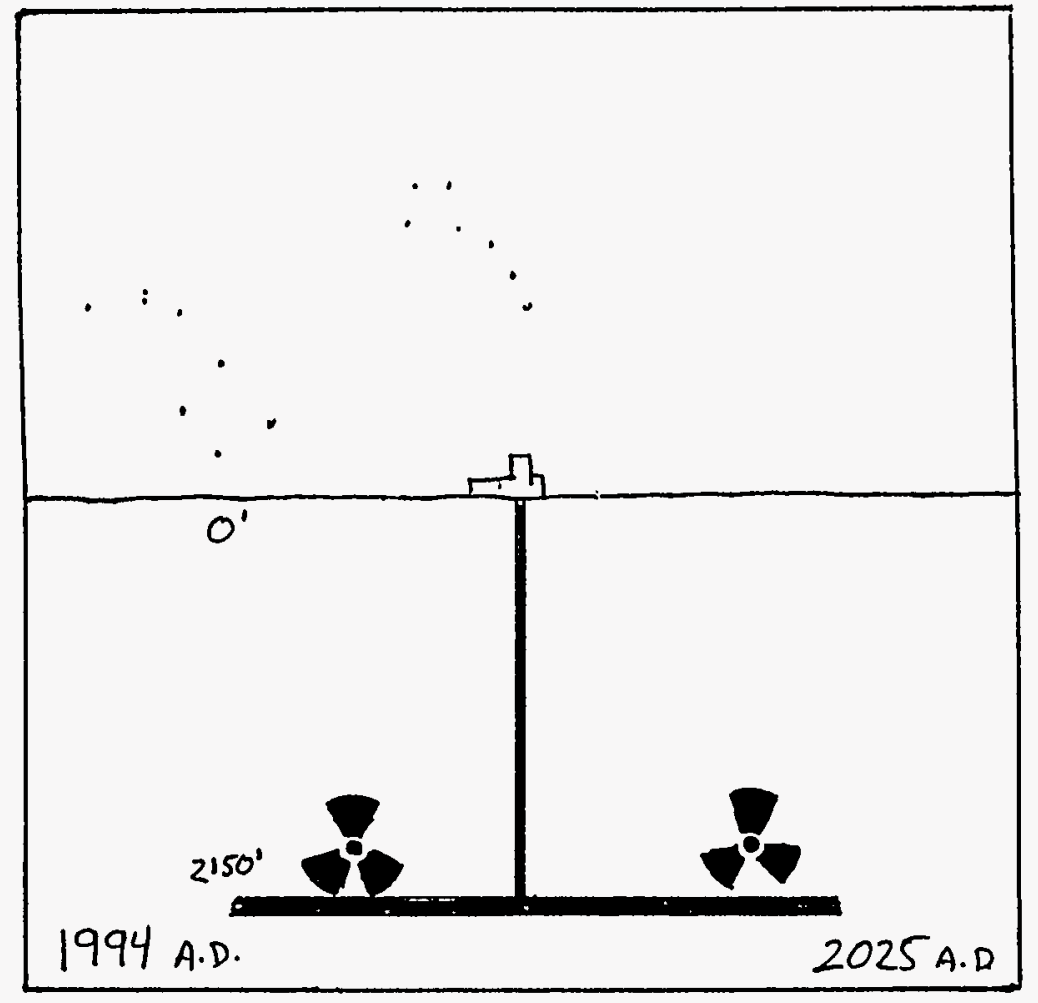

000000

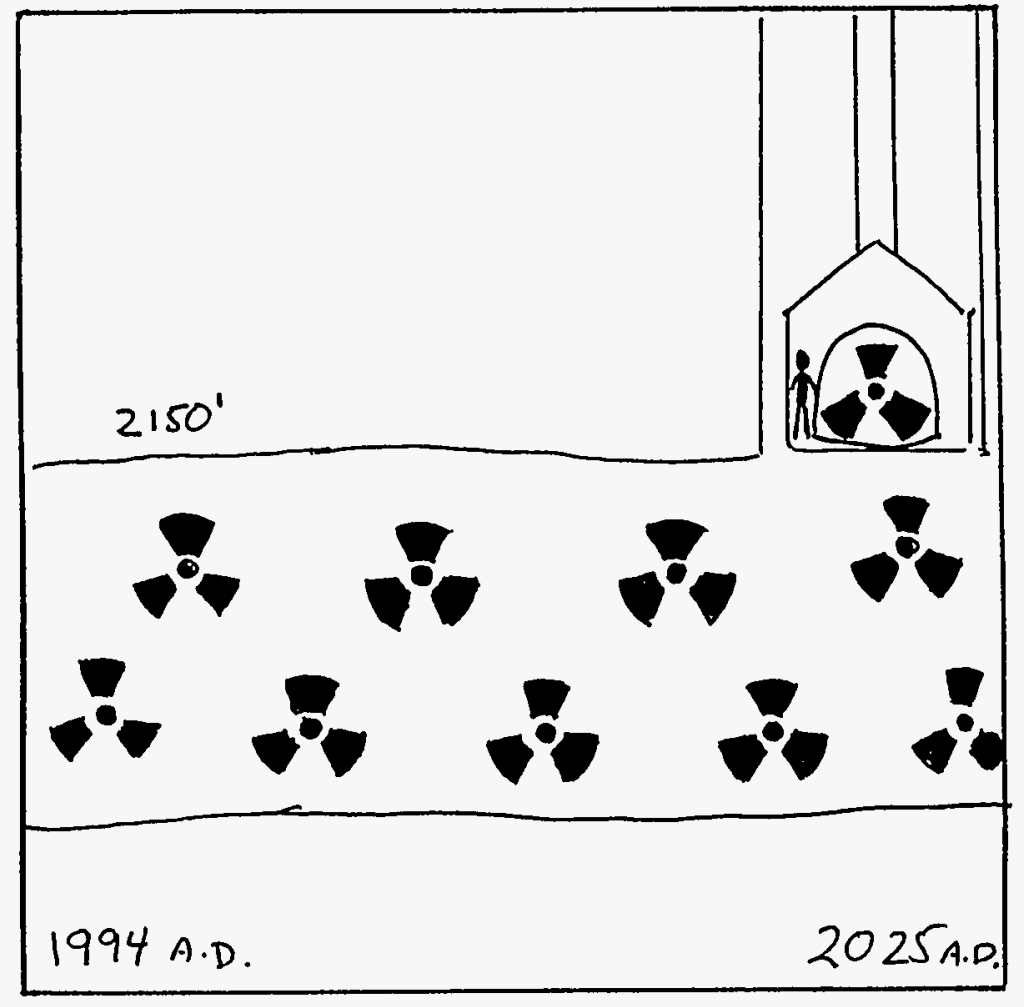

(g)

Figure 13. Pictographs showing the Passage of Time at the WIPP. Parts (a) through ( $j$ ) relate the passage of time with the number of dots, and present a closeup surface picture by a long-range surface and underground picture (axt by John Lomberg). (Figure continued on next page). 


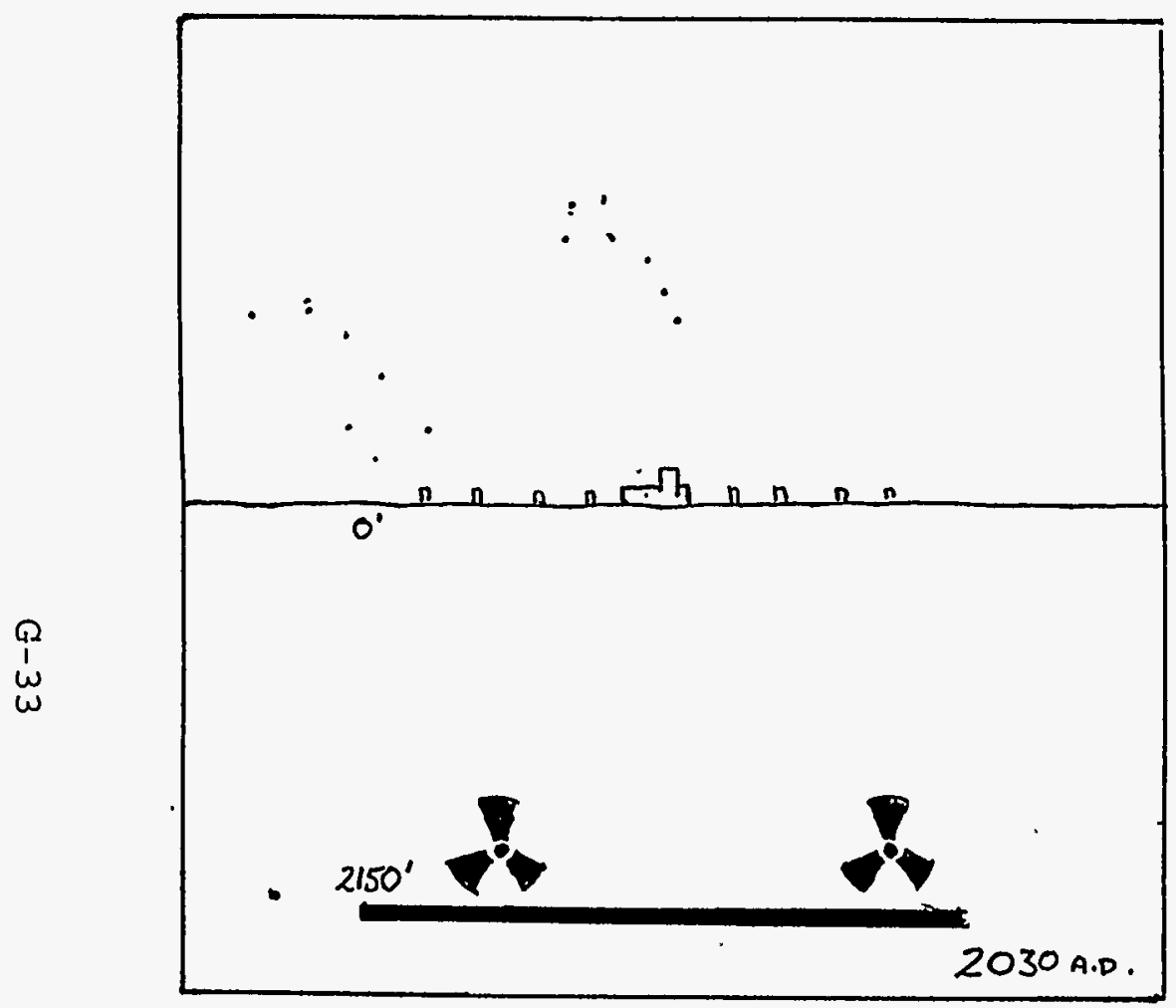

๑0000000

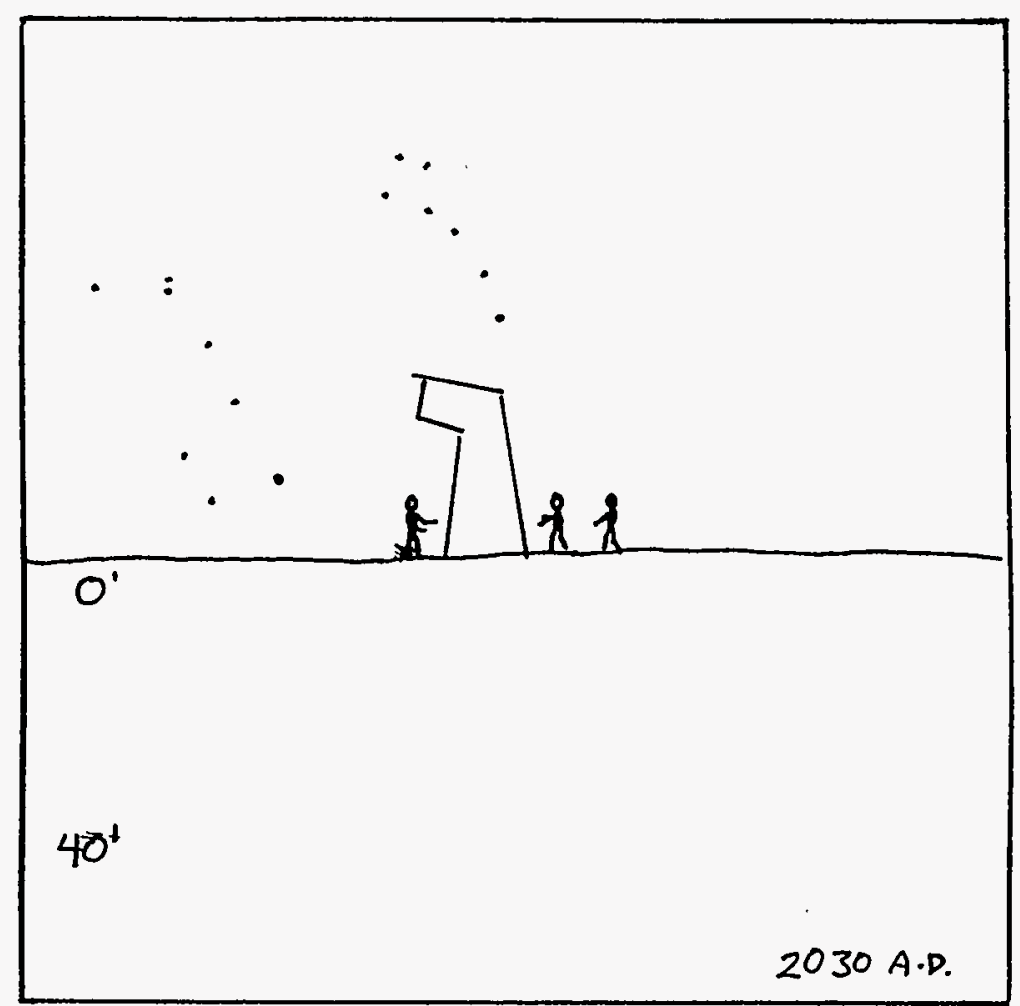

(h)

Figure 13. Pictographs Showing the Passage of Time at the WIPP. Parts (a) through (j) relate the passage of time with the number of dots, and present a closeup surface picture by a long-range surface and underground picture (art by John Lomberg). (Figure continued on next page). 


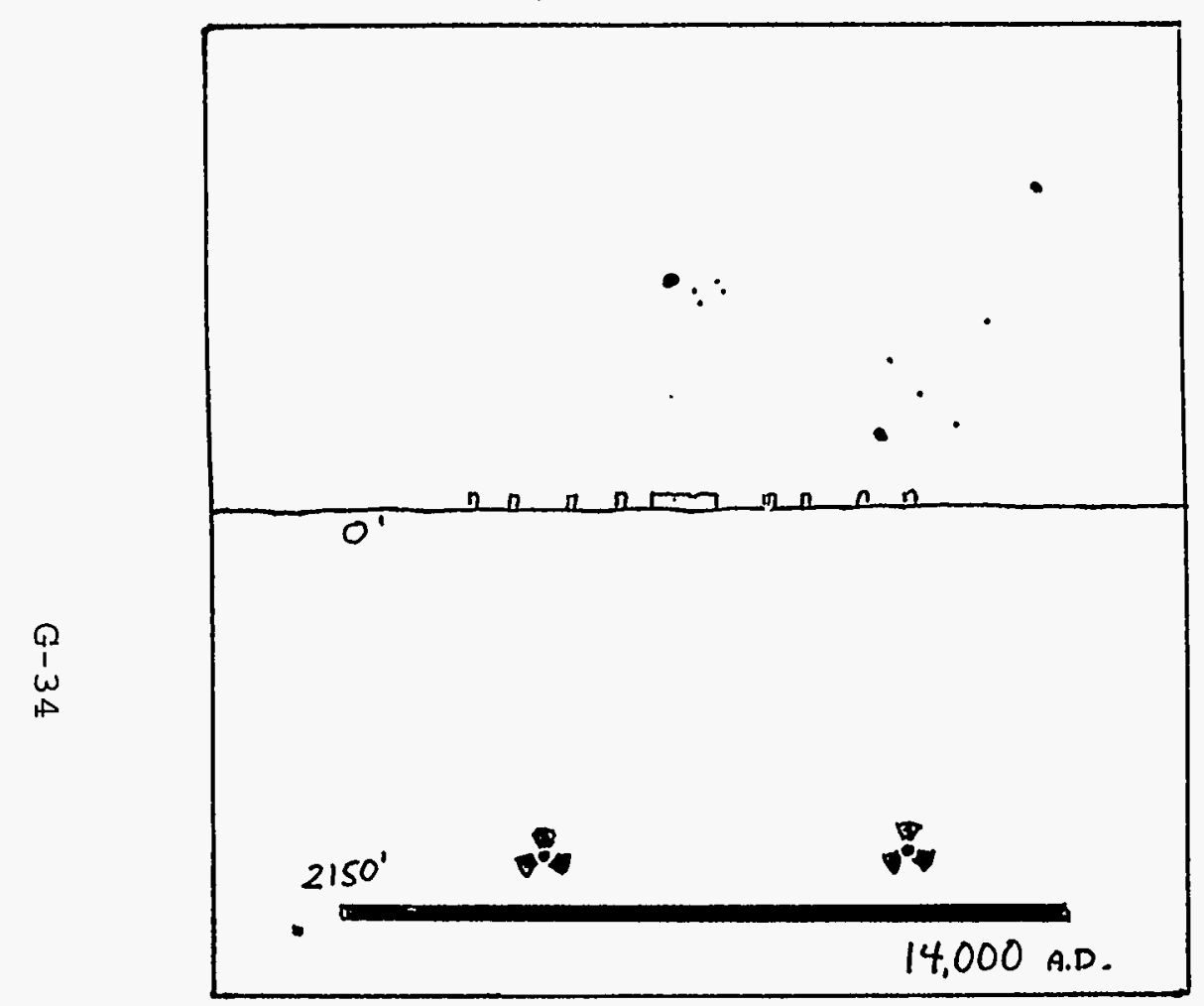

000000000

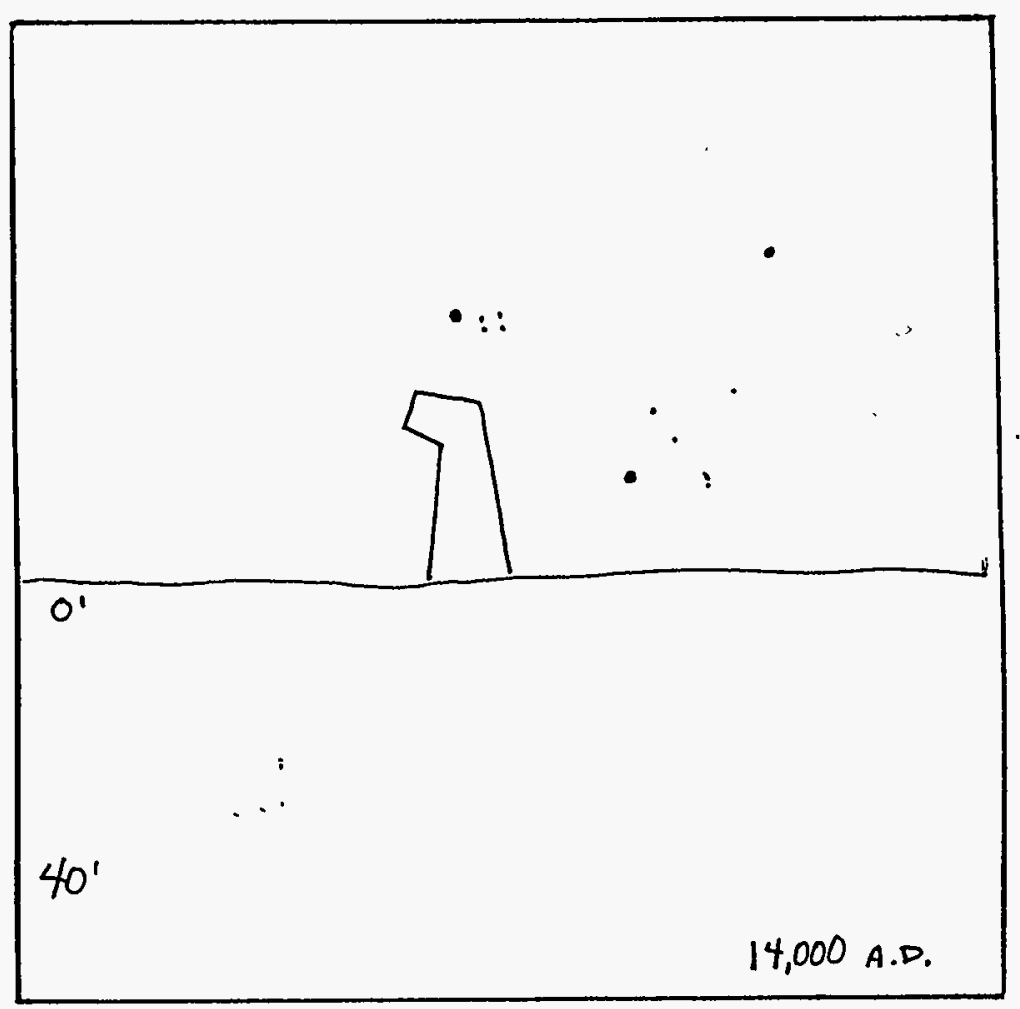

(i)

Figure 13. Pictographs Showing the Passage of Time at the WIPP. Parts (a) through ( $j$ ) relate the passage of time with the number of dots, and present a closeup surface picture by a long-range surface and underground picture (art by John Lomberg). (Figure continued on next page). 


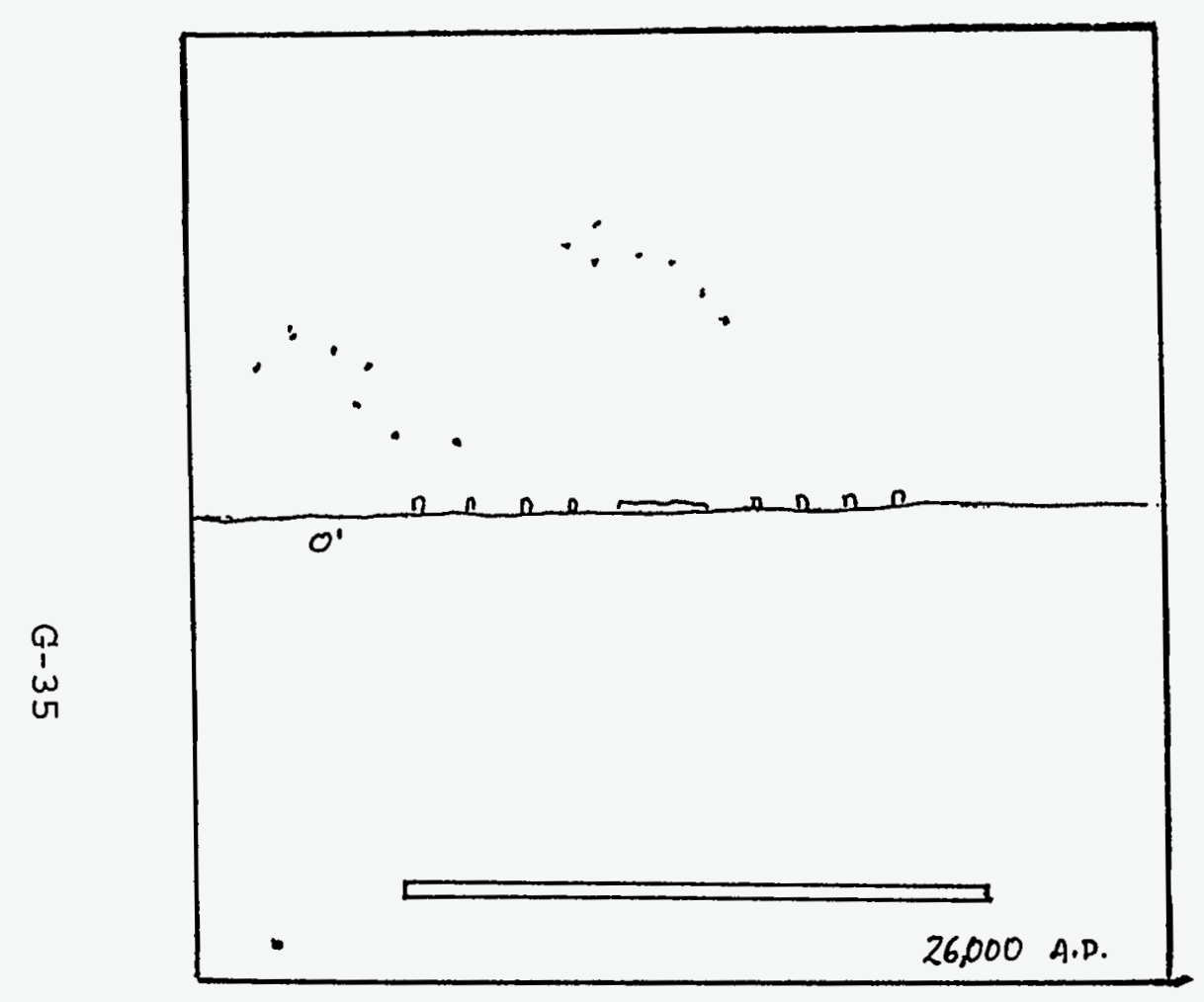

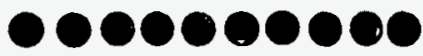

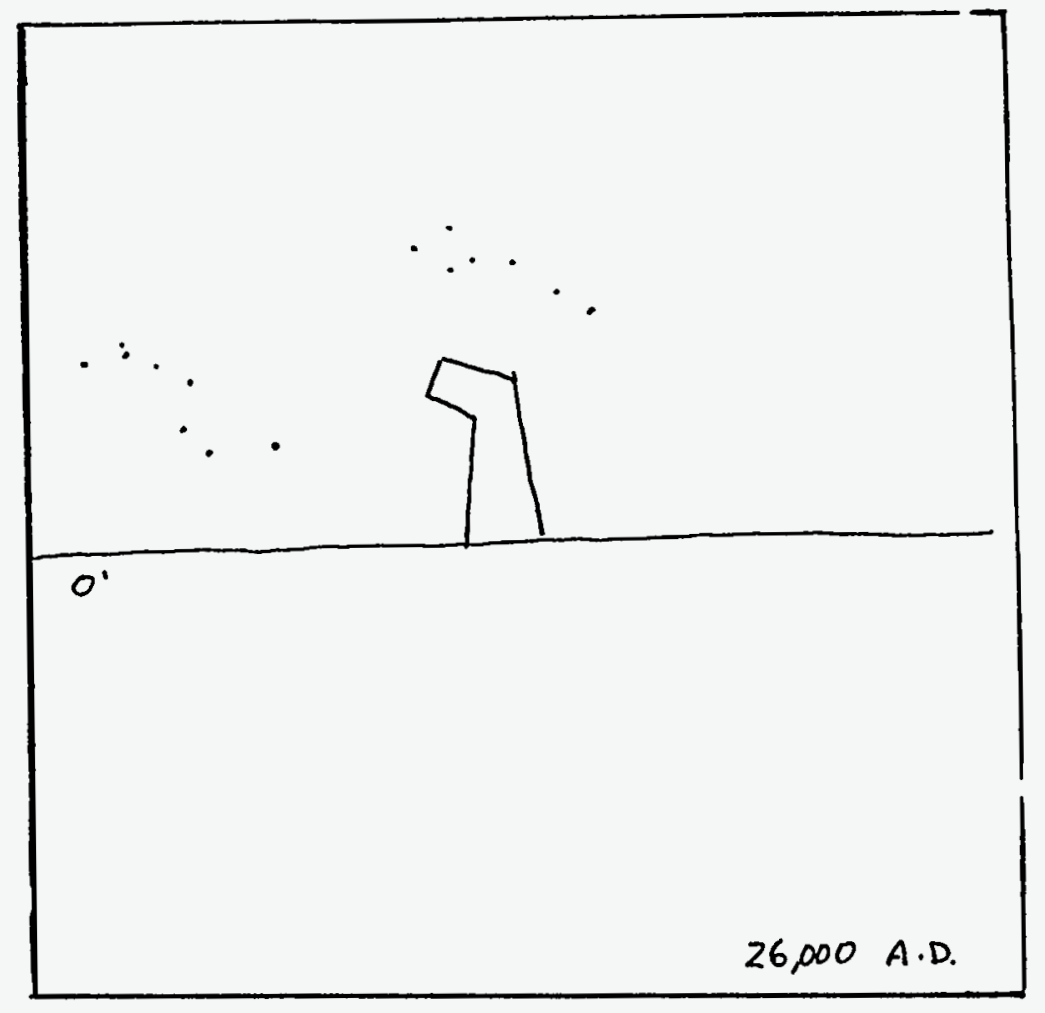

(j)

Figure 13. Pictographs Showing the Passage of Time at the WIPP. Parts (a) through (j) relate the passage of time with the number of dots, and present a closeup surface picture by a long-range surface and underground picture (art by John Lomberg). (Figure concluded). 
made by humans is here." The most important property of a level-I sign is its own existence. "Human made" would be suggested by the patterned shape-the unnatural syntax and negative entropy-of the earthwork, rock structures and inscriptions.

Level II: Cautionary Information. Elementary linguistic scripts and pictographic narratives would convey: "Warning, dangerous materials are buried below."

Level III: Basic Information. Level-III messages, including longer linguistic narratives, pictographic sequences, maps and simple diagrams would explain basic what, why, when, where, who and how information about the site.

Level IV: Complex Information. Highly detailed written records, scientific data and diagrams would be available at the site in inscriptions and buried "time capsules."

Celestial reference points would be included as level-IV information (1) to provide a chronological reference and (2) to give the site an astronomical dimension. The specific reference to Earth's movement through space and time (relative to the northern hemisphere's invariant constellations) would add an imaginative, celestial character that could help the site remain in society's memory. Precession (westward motion) of the equinoxes (the 26,000 year cycle) and the shape of the Big Dipper could give the site a chronology. From prehistory onward, humans have displayed an intellectual and emotional curiosity about their place in the cosmos.

As the noted astronomer, Edwin Krupp, stated: "For most of the history of humankind, going back to stone age times, the sky has served as a tool. Just as the hands of the first people grasped the flints they crafted, so their brains grasped the sky. The regularity of the motions of celestial objects enabled them to orient themselves in time and space" (Krupp, 1983, p. 1).

\section{Individual Marker Performance}

This report is structured in response to Sandia National Laboratories' request that we consider the probability of a marker system surviving and being understood within three time periods: 0-500, 500-2,000 and 2,000-10,000 years. Our design, however, evolved from more general discussions of the problem without reference to the specific time periods. We consider the following estimates to be reasonable yet subjective guesses. 
Indeed, the consensus of our team is that the probabilityconsensus elicitation required by sandia was the least certain part of our effort. We urge readers to evaluate our proposal on the merits of the design itself rather than on the accuracy of our probability estimates. Physical survival of the markers and clarity of the messages were our primary design criteria. We urge that the proposed physical materials and messages go through an adequate process of testing and refinement to insure the highest possible performance.

\section{Persistence.}

(A) Earthwork.

(a) 0-500 Years. A 0.8-kilometer diameter, 30' high earthwork of patterned shape would be likely to survive as a recognizable land feature for 500 years. The earthwork should be composed of "useless" material that would have a low probability of being mined in the future.

(b) 500-2,000 Years. Rectangular, earthen mounds and plazas built around 950 A.D. (Krupp, 1983) at Cahokia, a Mississippian Indian metropolis near St. Louis, have survived for 1,000 years. An earthwork on the WIPP site would be likely to survive for 2,000 years with its shape intact.

(c) 2,000-10,000 Years. Simple mounded earthworks (e.g., the chambered passage grave at Newgrange built by neolithic farmers in Ireland, ca. 3300 B.C.; [Krupp, 1983]), have survived longer than 5,000 years. The banks of the 350' circular ditch surrounding Stonehenge, built around 3,000 B.C. (Stover and Kraig, 1978), though considerably eroded (the inner bank, originally 6' high by 20' wide, is now one foot high [Hawkins, 1973]), is still visible after 5,000 years. Hundreds of earthen mounds built in the U.S. during the Burial Mound period (1000 B.C. to 700 A.D.; Le Mouel, 1991) are easily visible $1,300-3,000$ years later. There is reason to assume an earthwork purposely designed to last 10,000 years would survive at the WIPP site.

(B) Monoliths.

(a) 0-500 Years. Large, granite surface markers would have a high probability of survival over the near future. The granite itself would be highly durable in a relatively arid environment. The size of the monoliths would make their removal difficult. The problem of 
mining the granite would be reduced if future societies considered the monolithic arrangement a human monument worth preserving (like, e.g., Stonehenge).

(b) 500-2,000 Years. Granite monoliths would likely survive with their shapes intact for 2,000 years.

A representative granite monolith could be stored offsite in nearby Carlsbad Caverns as an indexical sign that would refer back to the WIPP marker system. The richly inscribed, off-site monolith would be available for interpretation as long as humans could visit Carlsbad Caverns. Persistence of such a sign would be likely over the 500-2,000-year span.

For redundancy, a second stone marker, similarly inscribed, could be emplaced as a granitic plug offsite, 10-15' underground in the main shaft of the WIPP. The stone seal would be inscribed with a sample of messages from the marker system, and with messages showing the system's existence relative to the seal as a datum point. The likelihood of a buried plug's persistence over 500-2,000 years-even if uncoveredwould be great.

(c) 2,000-10,000 Years.

Granite. Granite is composed principally of quartz, mica and feldspar. Minor minerals include fluorospar, tourmaline, garnet, topaz and ferrous minerals (Evans, 1972). Quartz is a crystalline form of silicon dioxide $\left(\mathrm{SiO}_{2}\right)$, commonly found as sand. Feldspar is a potassium, sodium, calcium or barium alumino-silicate (e.g., KAlSi ${ }_{3} \mathrm{O}_{8}$, known as orthoclase; Deeson, 1973). An example of mica is biotite, a hydrous magnesium iron aluminum-potassium silicate (Deeson, 1973).

Water is the most important single agent in causing the natural disintegration (weathering) of granite (Twidale, 1982). Because of its dense crystalline structure, intact granite is characterized by extremely low permeability, reducing the movement of water into the rock to such an extent that granite monuments have great durability when exposed to the surface atmosphere and drained of surface water.

The action of water on granite is greatly enhanced by prolonged exposure to soil or ground water. Weathering tends to concentrate in fracture zones, which are much greater in permeability than the intact rock (Twidale, 
1982). The principal chemical weathering process for most natural granite is hydrolysis, mainly of biotite and feldspar. Biotite in particular is easily hydrated when in contact with water. The resulting hydrobiotite exerts a physical expansion to which some investigators attribute the major role in granite weathering (Isherwood and street, 1976). Feldspar alters by hydrolysis to clay minerals, silica and metal cations in solution. The clay minerals can exert physical expansion in a similar manner to the hydrobiotite. Small point stresses between crystal grains will, over time, disrupt intact granite to grus, an accumulation of disaggregated mineral grains of nearly identical composition to the parent rock.

Grussification is extremely slow on exposed, welldrained granite surfaces. On time scales of concern to marker persistence, the process will only be important if water is in continuous contact with the rock. This would occur in the following circumstances: (1) surface water is allowed to pond or otherwise be retained on the exposed granite; (2) regolith or other unconsolidated material buries the granite and holds moisture in contact with it. Both circumstances would be exacerbated by climatic conditions that would increase available moisture.

The most rapid long-term grussification occurs in rock basins that enlarge to depths of a few tens of centimeters and widths of several meters over a few thousand years (Twidale, 1982). On the time scale of 10,000 years it is extremely unlikely that these processes would appreciably modify the basic structure of granite columns measuring 10-25 feet in height and 10-20 feet in diameter. However, surface inscriptions could be compromised.

When exposed to rainwatex containing dissolved carbon dioxide (to yield carbonic acid), feldspar is weathered to form the clay mineral kaolin. When the feldspar is dissolved, the adjacent grains of quartz, mica and other materials are loosened and eventually washed away. This effect is seen in Cornwall, England, where the clay pits containing kaolin also feature granite "pyramids" still in the process of being weathered (Evans, 1972). This illustrates the point that primary rocks from the earth's crust which are formed by igneous processes underground are not stable in the earth's atmosphere (Smith, 1981). However, the process of weathering is evident only on a geological time scale orders of 
magnitude longer than the 10,000 year period of regulatory concern for the WIPP.

Salt weathering. The most potent combination of weathering agents for construction stone is moisture and salt (Winkler, 1975). If water containing salt solutions can enter the rock, subsequent evaporation of the water will lead to crystallization. Crystallization pressure, thermal discontinuities, hydration pressure, and other processes may then disaggregate rock containing the salt impurity.

The WIPP lies in an area of high salt concentration. Wind from mine spoil piles transports dust with high salt content. Leached into the soil, this salt can be dissolved in soil and ground water. Burial of granite in salt-rich sediment introduces a potential for salt weathering of rock surfaces.

Sulfuric acid. Another potential weathering process is attack by sulfuric acid, which decomposes mica to leave silica in fine scales (Deeson, 1973). Such attack is not likely now, as acid rain from industrial pollution is not a current problem. A more likely source of acid rain might be from large power plants burning coal, as in the Four Corners plant at the junction of Arizona, New Mexico, Colorado and Utah. A six-year study by the University of IlIinois indicated threat by sulfuric acid would not be significant at today's level of sulphur output.

In exposed granite outcroppings, the main observable effect of weathering is to smooth the angles and accentuate the joints that form naturally during cooling of the molten mass from deep in the earth that becomes granite on solidifying (Evans, 1972).

In summary, concerning stability of granite markers at the WIPP site over 10,000 years, the amount of acid erosion would be negligible if the area remains dry and unpopulated, and minor if rainfall increases significantly and if human habitation and industrialization overtake the area. Some protection against wind-blown sand and rain containing higher than normal levels of carbonic acid and/or sulfuric acid could be afforded by incising the information deeper than the one centimeter observed on some monuments, and by providing a ridge of unpolished material around the edge of the inscribed area. 
Sand. Wind-blown sand has the potential to accumulate at the marker site. The dryness of climate, lack of vegetation and source of sand-sized particles are all important in facilitating sand mobilization by wind (Costa and Baker, 1981). Prominent coppice dune fields immediately southeast of the WIPP attest to the local importance of this process. The low relief of earthwork should facilitate continued sand transport through the site without accumulation. The monoliths might act as obstacles in the wind field, leading to the accumulation of shadow dunes on their leeward sides. Because of wide spacing, however, it is unlikely that such dunes would coalesce. They would remain as relatively low forms extending downward from the monoliths. Their distinctive pattern could contribute an additional marking attribute of the site, making its appearance unique in the region.

Eighteen of the original 30 shaped, 50-ton, 13.5' high sandstone monoliths of the Sarsen Circle of Stonehenge have been standing for 3,500-4,000 years (Hawkins, 1973). [Sarsen stone $=7$ on Mohs' scale of hardness; steel $=6.7$ (Stover and Kraig, 1978).] There is reason to assume that large, granitic stones-which are harder and more durable than sandstone-purposely shaped and positioned to remain upright for 10,000 years, would last longer than stonehenge. The likelihood of survival of each large stone marker, including the two located off-site, would be great.

(C) Central structure.

(a) 0-500 Years. A granite structure with inscriptions on planar surfaces of the inner walls could be the most durable feature of the marking system.

(b) 500-2,000 Years. The inscribed, granitic surfaces within the central "rock shelter" would weather less than the structure's outer, exposed surfaces.

(c) 2,000-10,000 Years. A stable stone structure built of intersecting walls of solid granite would have a good chance of surviving the 10,000 year time period.

(D) Inscriptions (Symbols, Iconic Pictographs, Linguistic Scripts, Narrative Arrangements and Complex Scientific Diagrams)

(a) 0-500 Years. There is little scientific evidence on the long-term durability of incisions cut in stone. 
Most studies have been done on marble tombstones less than 500 years old. Research is needed to determine the most durable incision for the granite markers.

(b) 500-2,000 Years. The monumental Behistun carving (520 B.C.) summarizing the biography of Persian King Darius I has lasted longer than 2,000 years. While inscriptions on buried monoliths would be highly likely to survive for at least 2,000 years, inscriptions exposed to weathering on surface monoliths would be expected to show signs of erosion from blown sand, carbonic acid in rain and perhaps sulfuric acid if coalfired power plants are built in the area. (Coal might not be used as an energy source during the 500-10,000 year time period.)

(c) 2,000-10,000 Years. Though faint, a carving of a square-hilted dagger on the inner surface of sarsen stone number 53 at stonehenge has survived in an open field for 3,500-4,000 years (Stover and Kraig, 1978). It is likely that stone incisions designed to endure for 10,000 years would last longer than the carved dagger at stonehenge.

(E) Buried Time Capsules.

(a) $\quad$-500 Years. Most materials endure longer if buried in dry sediments than if left to weather on the surface. Time capsules fabricated of materials expressly chosen for their durability underground would have a very high probability of surviving for 500 years. Molded and fired plates of aluminum oxide would be one possible choice.

(b) 500-2,000 Years. Dry-sand burials of human bodies in ancient Egypt preserved bone, tissue and organs more efficiently than later mummification and embalming techniques. The dry, desert environment of the Delaware Basin in southeastern New Mexico would not be expected to threaten materials buried in sandstone or mudstone formations. Alumina is a natural constituent of geological formations.

(c) 2,000-10,000 Years. Stone projectile points, bone needles and bison teeth have survived for 10,000 years underground in damp sands and clay at the Lind Coulee Paleoindian site in eastern Washington state. Camel teeth and bones have been found in ancient spring deposits not far from the WIPP itself (R.V. Guzowski, personal communication, Science Applications 
International Corporation, November 4-6, 1991, Albuquerque, NM) . Artifacts designed to endure in dry deposits would be likely to have a greater chance than bone needles of surviving 10,000 years.

Buried time capsules ranging in size from six inches to two feet in diameter, seeded beneath the surface of the earthwork, would most likely be found after thousands of years as the earthwork itself eroded, exposing those plates closest to the surface in a slowly-timed release. Future archeologists would be likely to find the artifacts in excavations or to sense their existence electronically. Buried time capsules and inscribed plates would be unlikely to be found by future drilling operations.

\section{Recognition}

(A) Earthwork.

(a) $0-500$ Years. The earthwork's geometric shape would be recognizable as long as enough remained for curious humans to imaginatively reconstruct. Archeologists have reconstructed ancient, seemingly obliterated hearths, post holes, building foundations and inscribed geometric shapes from the barest traces of material remains.

(b) 500-2,000 Years. Human curiosity regarding ancient earthworks, and creative thoughts about what their patterns signify, will likely persist for 2,000 years. The large, unnatural geometric shape at the WIPP isite would with a high degree of probability convey at least a level-I message (i.e., rudimentary information) that "Something made by humans-if only the earthwork itself -is here."

(c) 2,000-10,000 Years. The symbolic shape of the earthwork would be more recognizable if its design were repeated throughout the marker system in linguistic, pictographic and diagrammatic inscriptions, and in the buried time capsules. The chance of recognizing the earthwork's geometric shape 10,000 years from now would be greater than the likelihood of recognizing the pattern as a meaningful symbol.

Marking with High Dielectric Materials. Recognition of the earthwork could be enhanced through dielectric materials. Radar (an acronym for Radio Detection and Ranging) is widely used in modern remote sensing from aircraft and spacecraft (Henderson, 1985; Sabins, 1978). Side-looking, synthetic- 
aperture radars (SAR) have received extensive recent application in the U.S. Space Shuttle and Seasat Programs to study Earth (Ford et al., 1989) and the Magellan Mission to Venus (Pettengill et al., 1991). Operating in the microwave portion of the electromagnetic spectrum, radar systems are of great interest because of their ability to penetrate the atmosphere in nearly all weather conditions except heavy rain. Radar's active sensing can be used at night, making it useful for military reconnaissance. It is very likely that radar will continue to be used by advanced civilizations in the future to monitor the surface of Earth.

The strength of the return radar signal from the terrain surface to the radar antenna depends on characteristics of (1) the radar signal itself and (2) the terrain. Radar signal properties are easily measured in terms of (a) wavelength, (b) polarization and (c) incidence angle (which also depends on terrain slope). The key terrain properties are (a) surface roughness and (b) the dielectric constant of the surface.

Sandy, arid terrains have relatively smooth surfaces that do not provide noticeable anomalies or bright radar response. Moreover, flat landscapes, like that at the WIPP, will further enhance the uniformity of signal response. In such areas dielectric properties may become important. Dry rocks and soils tend to have very uniform, low dielectric constants of about 3 to 8 , while water can be as high as 80 (MacDonald and Waite, 1973). The dryness of the WIPP site ensures low values.

Materials with unusually high dielectric constants include metal sulfides (such as iron pyrite) and ferrimagnetic minerals (such as magnetite and pyrite). These are fairly common products of mining operations and are readily available in sand-sized form. Thus, it is feasible to mix such high dielectric materials with the surface soils of the WIPP to comprise a marker that would be highly visible to radar remote sensing.

(B) Monoliths.

(a) 0-500 Years. The rudimentary level-I message ("Something made by humans is here") would be evident in the circular arrangement of standing, worked monoliths. Moreover, the monumental configuration of large, shaped stones would connote, "Something important is here."

(b) 500-2,000 Years. Inscriptions on the monoliths would reinforce the stones' level-I message. 
Furthermore, with age the monoliths could become recognized as a preservable, historical resource. Should individual monoliths topple or be removed, the geometric consistency and numbering (as a power of two with, e.g., 32 monoliths in the circle) would be a likely indication of what was missing, and from where. (The number two is used because the dyad is mathematically basic and symmetrical; its use as an ordering device could be inferred even if a majority of monoliths were gone from the arrangement.)

(c) 2,000-10,000 Years. The ring of monoliths would be recognizable even if the majority of component stones were to break or be toppled. Should individual monoliths become unrecognizable, it is still likely that the patterned shape of the circular arrangement would persist for 10,000 years.

To establish a human dimension, the diameter of the ring of monoliths would approximate the length of a soccer, rugby or football field. Visitors within the ring would see all the monoliths, feel psychologically enclosed in the circle, become "involved" with the stone monuments, and be drawn around the circumference to examine pictographs and messages inscribed on the granite. The ring of monoliths could be designed to engage future humans as active interpreters and as guests.

\section{(C) Central structure.}

Probability is high that the granite structure emplaced at the center of the monolithic ring would be recognized as an intentionally constructed human artifact. Should the shelter itself collapse, observers from the future would still be able to infer that a fabricated structure once stood. The richly inscribed inner walls would be decipherable over the 10,000-year period, whether standing or collapsed. The presence of inscriptions, even if unintelligible, would convey the level-I message that "Something made by humans is here."

Central placement of the rock shelter would draw future visitors through the encircling earthwork and ring of monoliths to the center of the marker, where inscriptions inside would carry pictographic, Iinguistic, diagrammatic and scientific information. The designed shape itself would attract people to the structure, which they could easily enter to view the inscriptions overhead. Inside, they would also find information about-and directions for sighting-key constellations of the northern sky from within the shelter. 
The shape and orientation of the rock shelter will have to provide easy access to visitors while minimizing potential burial by wind-blown sand. Aerodynamics is a concern here. Although sand mainly occurs as thin sheets in this region, accumulation around vegetation (coppice dunes) can reach depths of six meters. Mobilization of this sand by reduction of vegetation (through climate change or human action) could lead to redistribution of sand at the marker site.

An aerodynamically streamlined shape allows sand to bypass a potential obstruction without accumulating (Greeley and Iversen, 1985). Any aerodynamical streamlining of the rock shelter should be carefully oriented relative to the prevailing wind direction. Another strategy might be to place the entrance behind a streamlined baffle on the upwind side of the granite structure. Then any sand accumulation in the lee of the structure will not obstruct the entrance.

The granite shelter would be the most interesting and complex marker within the system of markers at the WIPP. Purposely designed to be the world's longest-lasting human artifact, the likelihood of its recognition at least as a level-I message would be high across the 10,000-year span.

(D) Symbols.

Team B agreed that the WIPP marker system should include a symbol or symbol set.

According to Givens (1982, p. 176), "An international graphic symbol or emblem for biohazards should be put into general use. The emblem can provide a tangible focus for a simple oral transmission of information about hazardous substances, such as radioactive waste. The meaning of the symbol may be transferred across generations by including it as a component in iconic narrative material (i.e., the pictorial material would 'teach' the symbol's significance). A worldwide symbol could function as a unifying theme for the entire repository communication system."

(a) 0-500 Years. A symbol is a sign whose physical shape and significance (meaning or reference) is purely traditional. Without understanding the tradition and its cultural history, a symbol is virtually indecipherable. An example is the U.S. (or any national) flag. The probability of symbol recognition over time is, therefore, low. If used, the trefoil could easily lose its reference to nuclear radiation within 500 years. 
However, symbols are among the most powerful of human signs. Along with flags, other potent symbols include the Star of David and the Christian cross. Using the trefoil or a yet-designed geometric shape in a monumental earthwork, and repeating the design throughout the inscriptions and in the buried time capsules would help the chosen symbol become a recognizable "trademark" for the site. On-site linguistic, pictographic and diagrammatic inscriptions could be used to teach the symbol's meaning. Worldwide use in waste repositories would give the symbol a higher probability of being recognized as a sign marking buried nuclear waste and its danger.

(b) 500-2,000 Years. If indexed strategically throughout the marker system's sign modalities, a symbol would be more recognizable to future humans than if it appeared solely as the shape of an earthwork.

(c) 2,000-10,000 Years. There would be a fair probability that the symbol encoded in the surface earthwork could serve ultimately as a shorthand label for the WIPP site, just as the pyramid has become a symbol representing Egypt. Should this happen, the probability of recognition across 10,000 years would increase.

(E) Iconic Pictographs.

(a) 0-500 Years. An iconic pictograph is a sign whose physical shape and significance (meaning or reference) bears a direct, intuitive relationship to the physical shape of what it stands for. Decipherment is aided by an iconic sign's visible resemblance to its referent. Examples include the crescent moon, the smile face and the human stick figure. When a crescent-moon pictograph, for instance, is used to signify the crescent moon, the sign is highly iconic. Iconicity is lost, however, as the crescent shape takes on less obvious meanings, such as in marking an outhouse door.

When signs are designed explicitly to preserve the visual reference, their meanings across time are liable to be more recognizable. Airport pictographs of men, women, baggage, food and cocktails are examples of signs whose iconicity has been explicitly preserved for contemporary viewers. 
Team B agreed that there is a greater than 90 percent chance that simple pictographs could convey accurate information about consequences of intrusion into the repository. Specific pictographs and narrative sequences should be designed by international graphic symbol specialists and tested by behavioral scientists on people of diverse educational and cultural backgrounds.

(b) 500-2,000 Years. The iconic principle used in the WIPP markers is likely to aid sign recognition 2,000 years from now. Egyptian funerary art, for example, has conveyed complex information in graphic, pictographic form for 3,000 years (Figure 14). It is likely that explicitly designed pictographic signs, strategically targeting future humans, could achieve better recognition than ancient Egyptian pictographs.

(c) 2,000-10,000 Years. The imagined narrative scenes would show both what happened during construction of the WIPP and what would happen if intrusion were to occur. Information-rich pictographs have survived longer than 10,000 years. Spanish Levantine rock art, for example, dating back 12,000 years, still speaks to those willing and imaginative enough to reconstruct depicted narrative scenes of human hunting parties pursuing prey animals. Thus, there is a better than even chance that message designers consciously working to preserve iconicity and to enhance the narrative significance of pictographic messages could send recognizable meanings across the 10,000-year span.

(F) Linguistic Scripts.

(a) 0-500 Years. Recognition of written messages and warnings would be likely to persist for 500 years. For comparison, Modern English itself is about 500 years old.

(b) 500-2,000 Years. Easy recognition of written scripts in the middle future would be less likely because today's languages could by then have changed dramatically. Middle (c. I100-1500 A.D.) and Old English (c. 400-1100 A.D.) are virtually unreadable to most humans today. It is likely, however, that future scholars would have little trouble deciphering 20thcentury linguistic scripts 2,000 years from now.

(c) 2,000-10,000 Years. Using a rate of retention per 1,000 years for "basic" vocabulary terms of 81 percent 


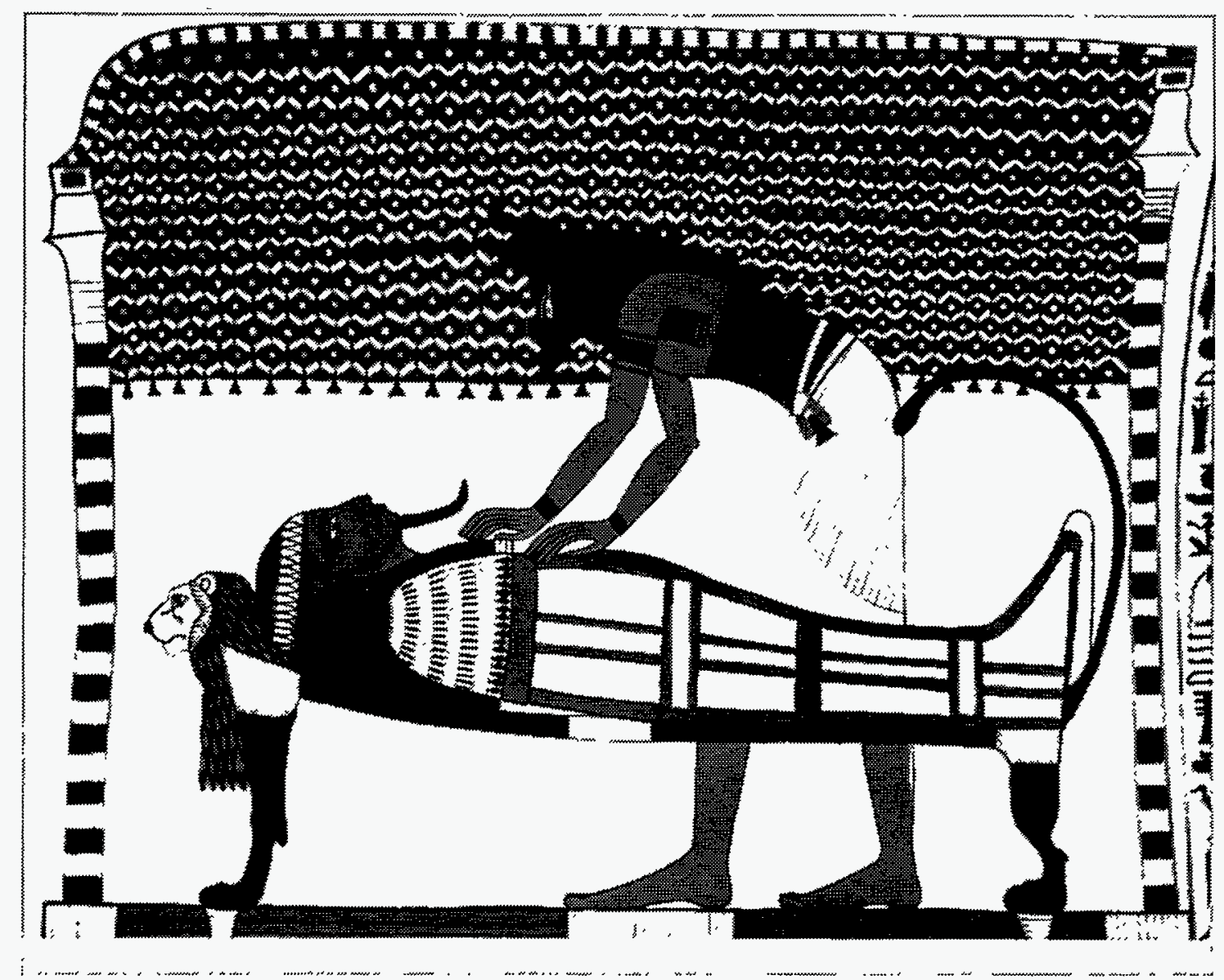

Figure 14. Egyptian Funerary Art (photo by M. McNaugher, The Carnegie Museum of Natural History). 
(a figure determined in lexicostatistics studies [Swadesh, 1952]), by 12,000 A.D. English will have retained as few as $12 \%$ of its current basic words, and still less of its more complex vocabulary items. Scholars may have a hard time translating very ancient scripts. But the likelihood of recognition could be increased by utilizing many scripts in the manner of the Rosetta stone. There is high probability that future classicists and linguists would, with scholarly effort, recognize and decode the writings.

The likelihood of decoding could be improved if written messages were designed using simple declarative sentences and a "basic" (Ogden, 1934), "monolexemic" (Swadesh, 1952) vocabulary that would be more likely to resist linguistic change over time.

(G) Complex Scientific Diagrams.

(a) 0-500 Years. Recognition of scientific diagrams such as the periodic table of the elements and nuclear reactions that produced the waste would be likely as long as major scientific paradigms remained similar to those that inspired the diagrams. Should radical shifts in scientific thought take place within 500 years, science historians and other academic specialists would still be likely to understand the periodic table.

(b) 500-2,000 Years. The periodic table would be more likely recognized by societies as advanced or more advanced than our own. Less advanced cultures would have trouble understanding the table and its scientific significance. To those who did recognize the periodic table (with the radioactive elements marked), its message would be: Danger, radioactive elements possible nearby. The graphic sign marking the radioactive elements would also appear, where appropriate, in iconic, linguistic, symbolic and other diagrammatic messages on site at the WIPP. Future readers would infer the sign's meaning from its occurrence within multiple contexts and message levels.

(c) 2,000-10,000 Years. Detailed maps and scientific diagrams, with their iconic references, would be more likely to be recognized by future societies, no matter how technologically advanced or simple they might be. A precise diagram of the surface markers could be used to communicate the exact scale (of their dimensions, distances and angles) proportionally to the materials buried below. 


\section{(H) Astronomical References.}

A Millennial Marking system. To ensure that generations far into the future, no matter how many disruptions in civilization and science might occur, could readily grasp when the WIPP was built and sealed, an astronomical way of dating the site should be built into the marker system. A pole star instrument or "millennial marker" could be constructed from a single monolith of granite. Based on an astronomical regularity known as the precession of the equinoxes, people from the future could date the site by sighting along the instrument and noting changes in positions of the "invariant" stars in the northern sky. Ten thousand years from now the earth's axis will be pointing away from the North star, Polaris, toward à position almost midway between the bright stars Deneb and Vega.

The Earth, like a top, wobbles as it spins. Slowly its polar axis traces a huge circle among the stars, a task requiring some 26,000 years to complete (Kyselka and Ianterman, 1976). To track the northern stars' positions over such vast periods of time, the millennial marker would be aligned to true north and its sighting ramp (Figures 15 and 16) inclined to the same angle as WIPP's 32 degree $23^{\prime}$ north latitude. Precession of the equinoxes and the westward drift of the vernal (spring) equinox through the zodiac could be depicted in language-free stone engravings and applied with a simple monolith serving as the sighting instrument.

(a) $0-500$ Years. In 1727 the Maharaja Jawai Jai Singh directed his craftsmen to construct a Pole star instrument, a long, narrow slab of red sandstone that still can be seen standing in the famous stone observatory of Jaipur, India (Singh, 1978; 1986). The craftsmen beveled the top of this narrow slab of sandstone so that it sloped upward at 27 degrees. Jaipur is located at 26 degrees $55^{\prime}$ north latitude. Because the angular height of Polaris above the horizon is approximately the same as the observer's latitude, one need only peer up the slope to see the North Star. Because Polaris is likely to persist as an important reference point for navigators for 500 years (at least), the proposed millennial marker has a very good chance of being recognized in the near future.

(b) 500-2,000 Years. In 2,000 years the North star would be sufficiently out of alignment with the millennial marker for future archeologists and astronomers to infer the passage of time. The chance of the divergence being recognized would be high; 


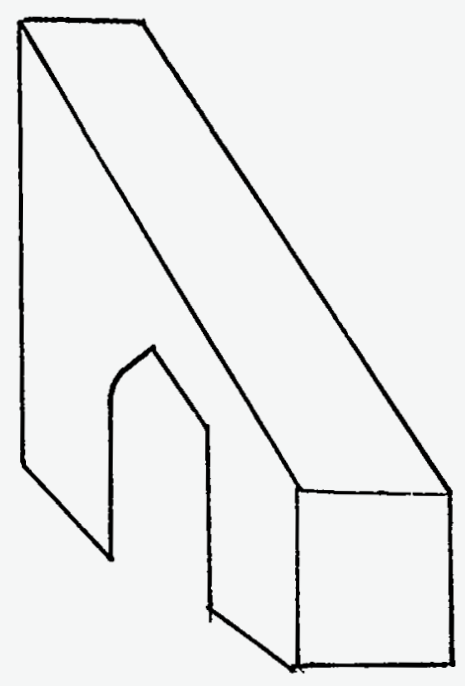

THE DUHRVA-DARSHAK YANTRA, OR POLE-STAR INSTRUMENT

BUILT IN 1727 BY THE MAHARAJA JAWAI JAI SINGH AT HIS STONE OBSERVATORY IN JAIPUR, INDIZ. THE INSTRUMENT POINTS TO TRU NORTH, AND THE TOP SLOPES UPWARD AT $27^{\circ}$ (THE LATITUDE OF JAIPUR) SO THAT IT SIGHTS DIRECTLY TOWARD POLARIS (FROM PRAHLAD SINGH, 1986 JANTAR-MANTARS OF INDIA: STONE OBSERVATORIES: JAIPUR, DELHI, UJJAIN, VARANASI, MATHURA. JAIPUR, INDIA: HOLIDAY PUBLICATIONS. 40.

Figure 15. The Duhrva-Darshak Yantra, or Pole-Star Instrument 


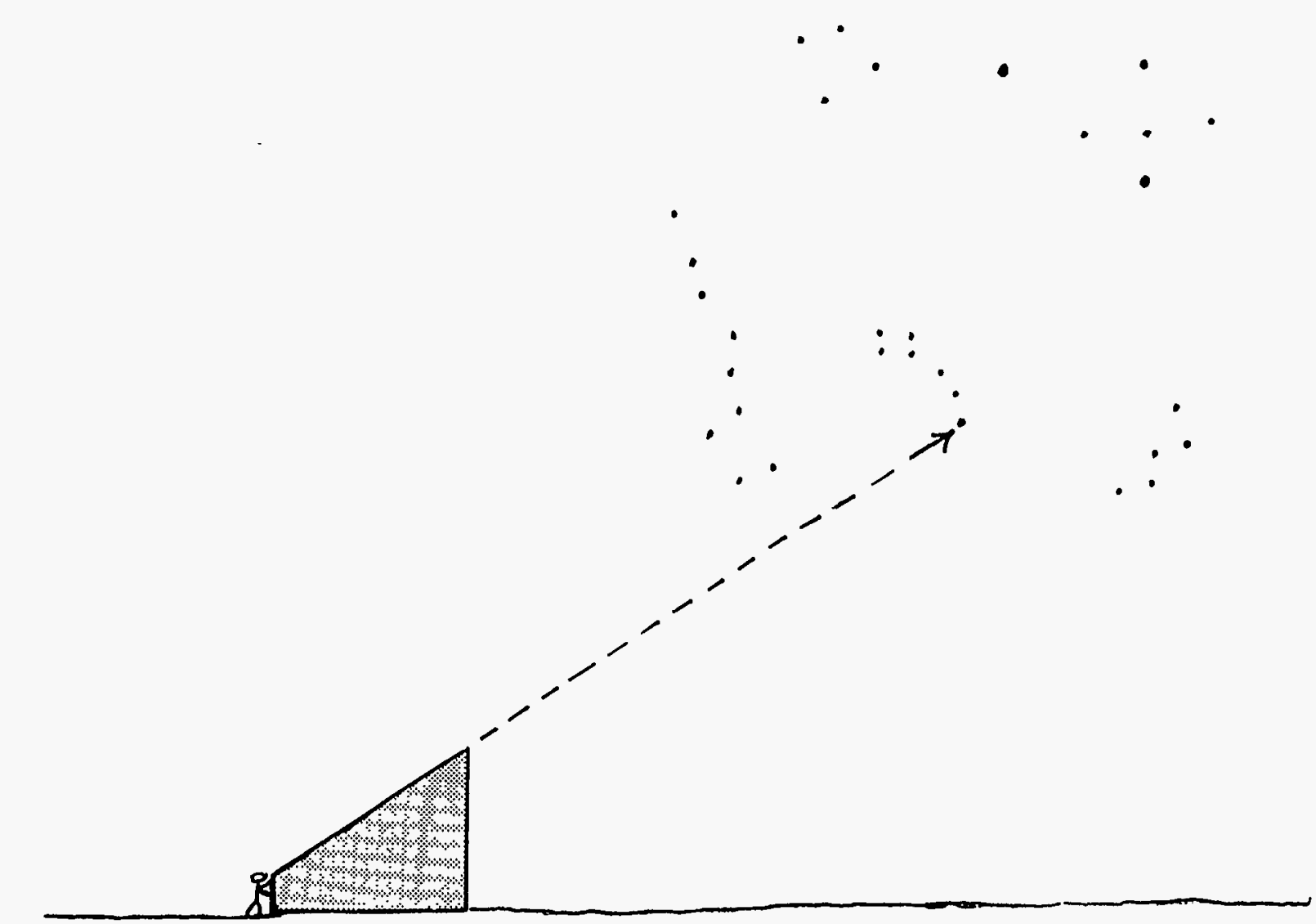

MILLENNIAL MARKING SYSTEM: 2,000 A.D. (0 Post Closure)

BY SIGHTING OVER THE TOP OF A LONG, NARROW STRUCTURE, WHICH IS ALIGNED ON TRUE NORTH AND INCLINED AT THE SAME ANGLE AS THE LATITUDE OF WIPP, THE OBSERVER OF 2,000 A.D. (GIVE OR TAKE SEVERAL CENTURIES) WILL BE LOOKING DIRECTLY AT POLARIS, OUR CURRENT "NORTH STAR."

(a)

Figure 16. Millennial Marking system. Parts (a) through (e) show the different locations of the stars as sighted by a pole-star located at the WIPP over 10,000 years (art by Ben Finney). (Figure continued on next page.) 


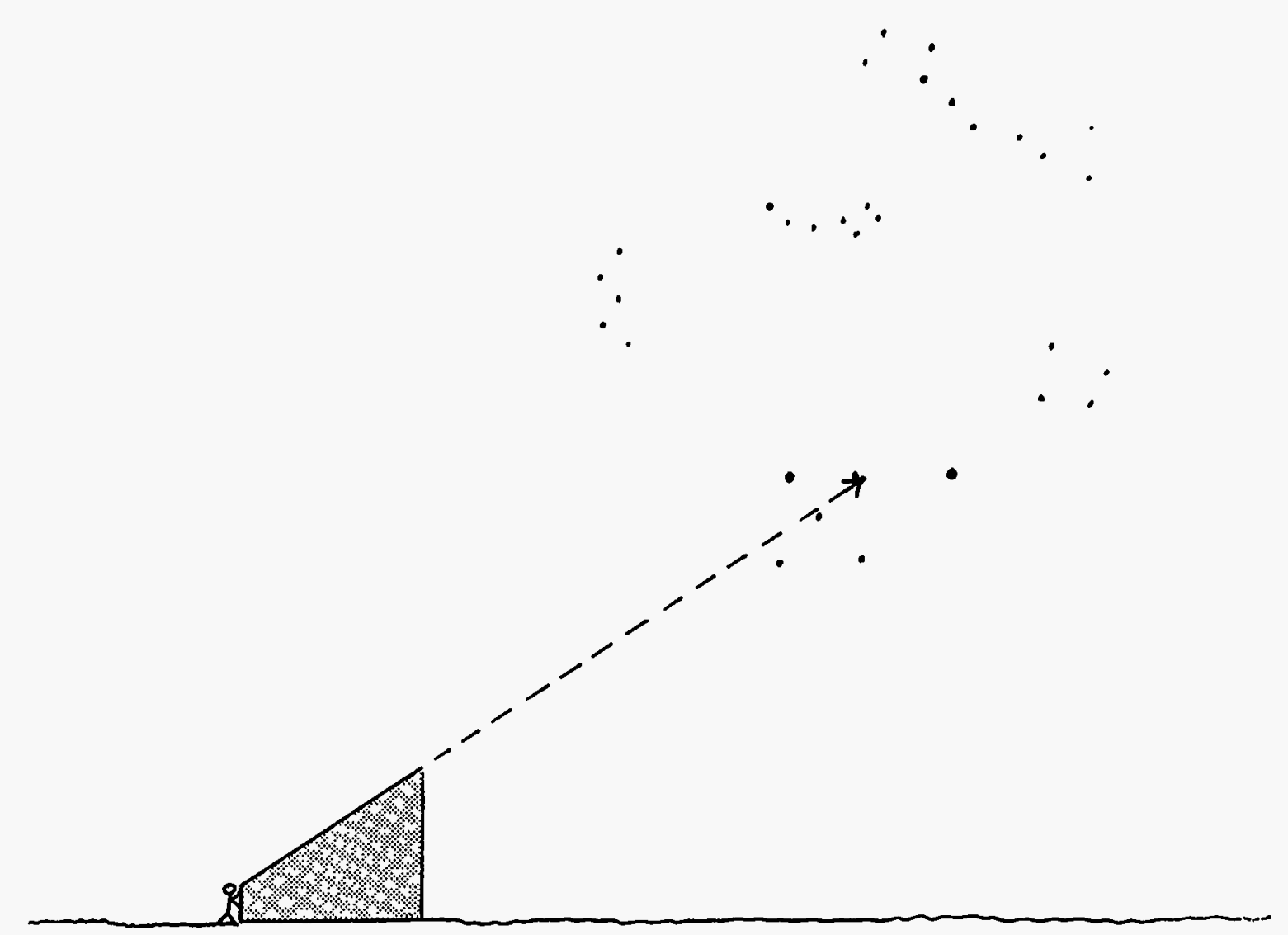

MILLENNIAL MARKING SYSTEM: 12,000 A.D. (10,000 P.C.)

THE WOBBLING OF THE EARTH AS IT SPINS CAUSES AN EXTENSION OF ITS AXIS TO TRACE A CIRCLE COUNTER-CLOCKWISE AMONG THE STARS OVER THE NORTHERN POLAR REGIONS. IN 10,000 YEARS (12,000 A.D.) AN OBSERVER SIGHTING TRUE NORTH OVER THE TOP OF THE STRUCTURE WILL BE LOOKING AT A POINT ROUGHLY MIDWAY BETWEEN THE BRIGHT STARS DENEB AND VEGA.

(b)

Figure 16. Millennial Marking System. Parts (a) through (e) show the different locations of the stars as sighted by a pole-star located at the WIPP over 10,000 years (art by Ben Finney). (Figure continued on next page.) 


$$
\begin{aligned}
& Q \\
& 1 \\
& \text { ind }
\end{aligned}
$$

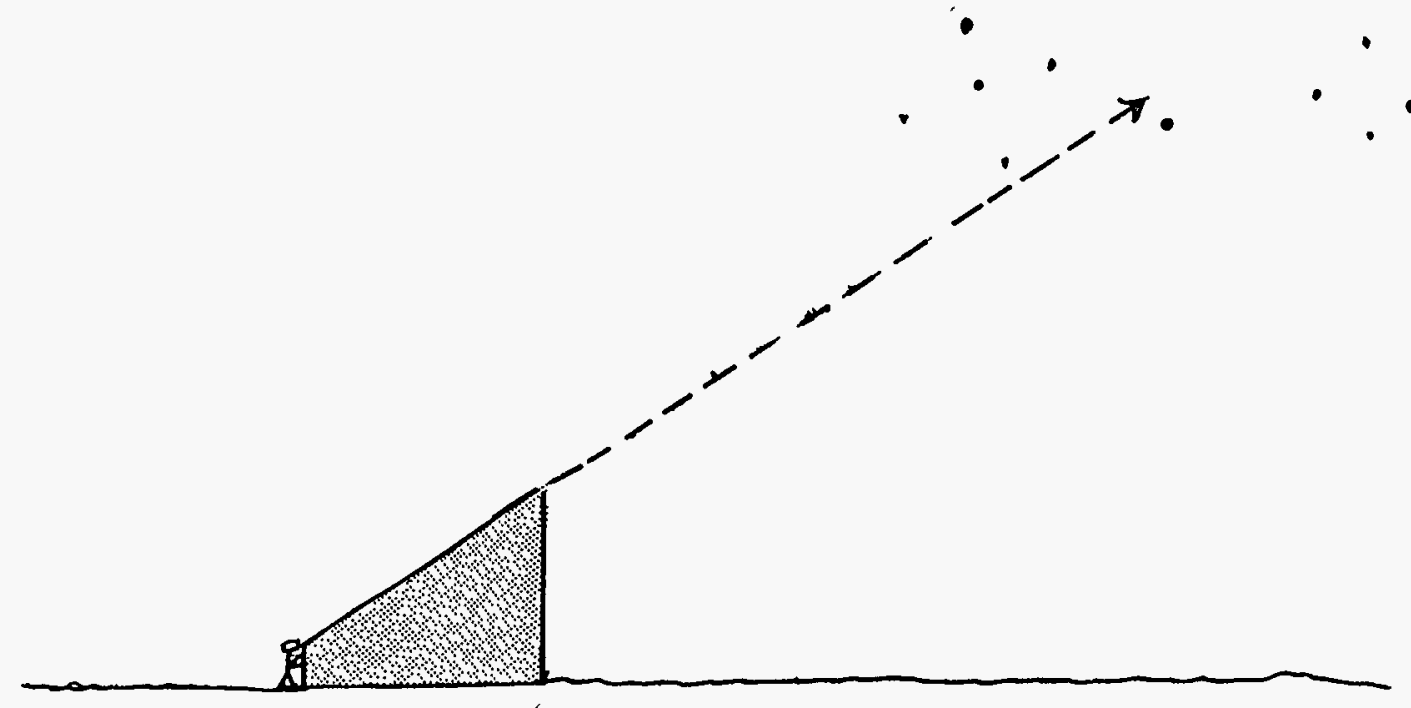

MILLENNIAL MARKING SYSTEM: 14,000 A.D. (12,000 P.C.)

AT 14,000 A.D. AN OBSERVER SIGHTING TRUE NORTH OVER THE STRUCTURE WILL BE LOOKING AT A POINT NEAR VEGA.

(c)

Figure 16. Millennial Marking System. Parts (a) through (e) show the different locations of the stars as sighted by a pole-star located at the WIPP over 10,000 years (art by Ben Finney). (Figure continued on next page.) 


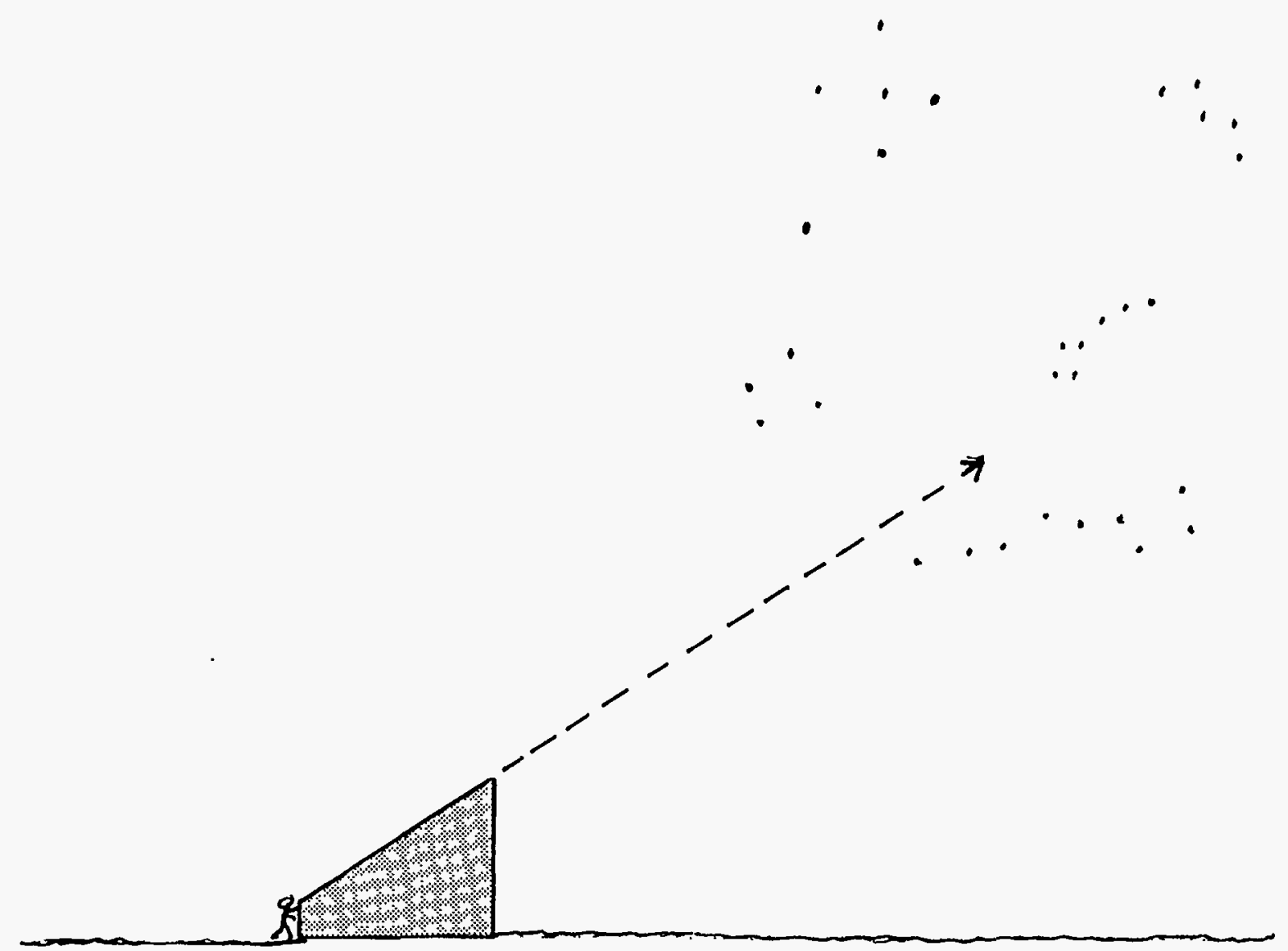

MILLENNIAL MARKING SYSTEM: 22,000 A.D. (20,000 P.C.)

AT 22,000 A.D. AN OBSERVER SIGHTING TRUE NORTH OVER THE STRUCTURE WILL BE LOOKING AT A POINT BETWEEN THE BIG AND LITTLE DIPPERS.

(d)

Figure 16. Millennial Marking system. Parts (a) through (e) show the different locations of the stars as sighted by a pole-star located at the WIPP over 10,000 years (art by Ben Finney). (Figure continued on next page.) 


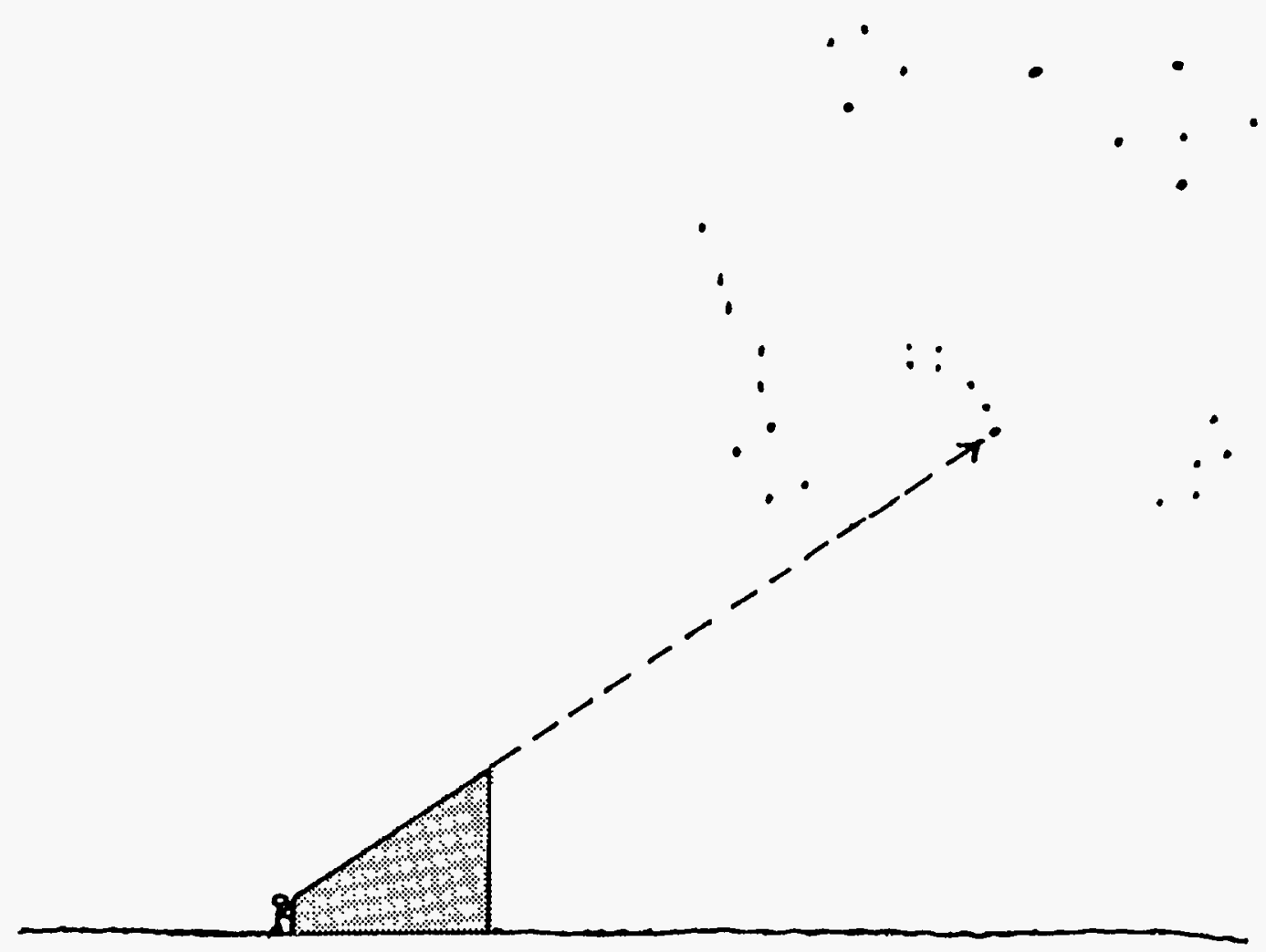

MILLENNIAL MARKING SYSTEM: 28,000 A.D. (26,000 P.C.)

AT 28,000 A.D., ONE 26,000 YEAR CYCLE OF THE PRECESSION WILL HAVE BEEN COMPLETED, AND AN OBSERVER SIGHTING TRUE NORTH OVER THE STRUCTURE WILL BE LOOKING TOWARD POLARIS, WHICH ONCE AGAIN WILL BE EARTH'S "NORTH STAR."

NOTE: THE SKEWING OF THE CONSTELLATIONS AND ASTERISMS BECAUSE OF THE DIFFERENT PROPER MOTIONS OF THEIR CONSTITUENT STARS IS NOT SHOWN HERE. BY SHOWING THIS IT WOULD BE POSSIBLE TO EXTEND THE CALENDAR BEYOND 28,000 A.D. AS THE POSITION OF THE STARS WOULD CHANGE SIGNIFICANTLY FROM ONE PAECESSIONAL CYCLE TO THE NEXT.

(e)

Figure 16. Millennial Marking system. Parts (a) through (e) show the different locations of the stars as sighted by a pole-star located at the WIPP over 10,000 years (art by Ben Finney). (Figure concluded.) 
precession of the equinoxes has been noted by many cultures worldwide for thousands of years.

(c) 2,000-10,000 Years. In 10,000 years the Earth's axis will be pointing away from the North star to a position midway between the bright stars Deneb and Vega. Should major discontinuities in knowledge of the current B.C./A.D. system occur in the remote future, it is reasonable to assume that the millennial marker, in tandem with explanatory diagrams and pictographs, could be used to recognize the WIPP's date of closure and to determine when it is "safe." Great care would need to be taken to make this marker stable.

Many peoples through the ages have used the nightly rotation of circumpolar stars and constellations to tell time. The Mescalero Apache, for example, still time the commencement and duration of pre-dawn rituals by such a star clock (Farrer, 1991). Although we do not know of cultures that have employed the precession of the equinoxes to keep track of the millennia, there are indications in ancient myths and religions that this slow shifting of stars was not only recognized by cultures reaching back perhaps as far as Paleolithic times, but also was the cause of great wonder (Desantillana and von Dechend, 1977; Ulansey, 1989; Worthen, 1991). It would seem likely, therefore, that even if major breaks occurred in civilization and science, people in future cultures across the next 10,000 years would still be able to recognize, interpret and understand the proposed millennial marking system.

\section{Interpretation}

(A) Earthwork.

(a) $0-500$ Years. Given that the earthwork is recognizable, the likelihood of correct interpretation as a level-I message ("Something made by humans is here") over 500 years would be great. Recognition of the earthwork's geometric shape as a symbol-and recognition of its 20th-21st-century meaning would be less likely.

(b) 500-2,000 Years. At 2,000 years the earthwork would suggest construction by humans "long ago." The "human- made" message would be likely to persist with a high level of probability. 
(c) 2,000-10,000 Years.

By 10,000 years the interpretation would be that the feature was constructed by "ancient" humans. There is nothing to suggest that future generations would attribute the earthwork's patterned shape to natural forces, geomorphological processes, animals or extraterrestrials. By itself, the 21st-century's symbolism would be unlikely to survive without cross-referenced clues to meaning elsewhere in the marker system's iconography, texts and scientific diagrams.

\section{(B) Monoliths and Central structure.}

The worked stones and their arrangement, along with the placement of a rock shelter at the exact center of the circle, would reinforce the earthwork's level-I message ("Something made by humans is here"). The probability that level-I messages would be correctly interpreted should be high across the 10,000-year span.

(C) Symbols.

(a) 0-500 Years. A designated warning symbol ideally would serve as the marker's "trademark." Successful indexing and cross-referencing with other on-site messages would help convey the symbol's contemporary connotations of "danger" and "warning." The warning symbol's late 20th-century meaning as a sign for nuclear radioactivity would be a difficult message for nearfuture viewers to interpret correctly. Still, in 500 years a fair probability exists that the symbol could become a popular logo for the site.

(b) 500-2,000 Years. The likelihood of the symbol's correct interpretation as a shorthand trademark or logo for the WIPP site, including connotations of danger and warning, could be enhanced if the trefoil (or a yet designed symbol) were used in many nuclear-waste repositories throughout the world.

(c) 2,000-10,000 Years. The symbol would be likely to increase in potency the longer it resided in society's memory. Assuming recognition is not a problem, its correct interpretation as a trademark for the marker system would be probable across the 10,000-year period. The planned association and cross-referencing of the symbol with other on-site messages could increase the likelihood of its correct interpretation as a level-II (cautionary) sign. 
(D) Iconic Pictographs.

(a) 0-500 Years. Pictographic messages designed according to principles of isotype (Neurath, 1936) and scientific illustration (Hogben, 1949) for remote-future addressees would have a high probability of correct interpretation in 500 years.

(b) 500-10,000 Years. Pictographic reference to environmental objects likely to be seen in the future, such as the human hand, face and body; the Sun, crescent moon and constellations; and the marker's earthwork and arranged monoliths themselves would be clearly meaningful in the midale and far futures. Pictographic narratives based on such signs would have a high probability of being correctly interpreted across 10,000 years.

A pictographic narrative could be used, for example, to depict the gradually changing shape of the Big Dipper (Figure 17), which would provide a chronological framework for the WIPP site.

Two narrative sequences, a simple "consequential" statement and a more detailed, historical depiction could be used. The former would be a three scene panel depicting (a) a human figure standing upright, then (b) ingesting a substance (perhaps small capsules marked with the designated warning symbol), and finally (c) lying down with rips and skull exposed. The message is, "This substance (whatever it may be) kills." The fact that humans cannot actually see what the nuclear sign represents will be less of a problem for future scientific societies that can decipher the periodic table of elements that it will be for less advanced societies. Still, humans need not actually see a deadly virus, "germ" or "spirit" in order to avoid the diseasecausing agent.

The historical sequence would show the site's construction through a longer series of pictographic panels, in narrative order from top-to-bottom (Figure 13). Properly drawn, there is good reason to predict that the messages would be correctly interpreted.

(E) Linguistic Scripts.

As stated above, though unreadable to most people 2,00010,000 years from now, there is high probability that future classicists and linguists could, with scholarly effort, 
THE CHANGING SHAPE OF THE BIG DIPPER OVER 100,000 YEARS

(FROM ROBERT JASTROW, ASTRONOMY: FUNDAMENTALS AND FRONTIERS. WILEY, NEW YORK, 1977, P. 1-35)

\section{THE BIG DIPPER TODAY}

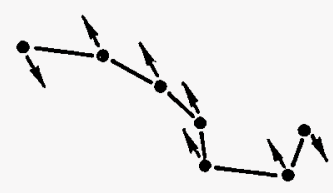

THE BIG DIPPER 50,000 YEARS AGO

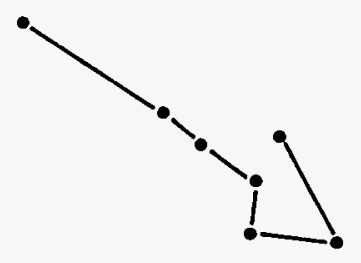

THE BIG DIPPER 50,000 YEARS HENCE

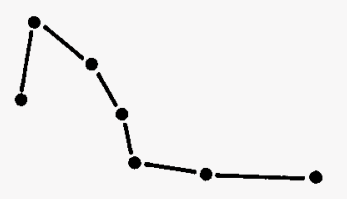

Figure 17. The Changing Shape of the Big Dipper Over 100,000 years (Jastrow and Thompson, 1977). 
recognize and decode the inscribed, written texts. Given the precision of writing as a form of communication, there is good reason to assume that written messages would be interpreted correctly 10,000 years from now.

(F) Complex Scientific Diagrams.

(a) 0-500 Years. Because of their precision and high information content, scientific diagrams recognized as such would have a very good chance of correct interpretation in the future.

(b) 500-2,000 Years. Their significance would be most meaningful to future scientists and/or science historians from societies as advanced, or more advanced, than our own.

(c) 2,000-10,000 Years. Assuming that human society continues to ascend the ladder of science and technology, as it has for the past 10,000 years since the domestication of plants and animals, there is a high probability that complex scientific diagrams would be correctly interpreted.

\section{Deterrence}

We cannot guarantee that any simple or complex message, even when recognized and correctly interpreted, will deter a human being from inappropriate action. The caution on tobacco products sold in the U.S. demonstrates how frequently people ignore explicit health warnings. Ironically, some messages (such as "Danger-750 Volts," painted on Washington, D.C.'s Metrorail tracks) lure the reckless and suicidal. Nevertheless, carefully designed warnings could be expected to reduce the chances of inadvertent intrusion into the WIPP. Moreover, an intrusion would not be casual, but would be a planned event. As such, there would be a greater likelihood to consider cautionary data.

\section{(A) Earthwork.}

(a) 0-500 Years. With respect to materials buried $2,100^{\prime}$ below the surface, an earthwork would offer two modes of deterrence: (1) as a monumental sign of longago or ancient human activity, and (2) as a symbol for dangerous materials: Regarding the former, presence of a large, geometric "mound" would not deter mining exploration, unless the mound had become a site of historic interest (a possibility, at least). The earthwork would be massive enough that even several exploratory efforts to "find something" in it would do 
little harm. Regarding a symbolic message-given that it had been recognized and correctly interpreteddeterrence and appropriate action would be likely across the near, middle and far future time periods.

An intentional goal of building a large, patterned earthwork would be to lock the site in society's memory. The earthwork's monumental size would be likely to help reduce the chance of forgetting its existence.

(b) 500-10,000 years. There is significant probability that an earthwork of great size and patterned shape would become better known with the passage of time, just as the world's ancient monuments have become increasingly known and recognized through the millennia. In the middle and far futures, therefore, an earthwork's potential to deter inadvertent intrusion actually could be enhanced.

(B) Monoliths and Central structure.

(a) 0-500 Years. As a monument commemorating the 21st century's concern for the safety of future generations, the ring of monoliths, the central rock shelter and the accompanying inscriptions would be interesting enough to remain securely in societal memory for at least 500 years.

(b) 500-10,000 Years. Deterrence as a monumental sign and warning symbol from 500-10,000 years in the future would be similar to that of the proposed earthwork (discussed above).

(C) Symbols.

A correctly interpreted symbol could provide high probabilities of deterrence. The symbol, in and of itself, would not provide needed when, what, where, how and why information to back up the warning.

But as a psychic trademark for the nuclear-waste repository, the symbol would increase the likelihood of deterrence and appropriate action. Should the symbol be used internationally, deterrence would be higher. 


\section{Marker System's Performance}

The probabilities and performance characteristics proposed above for the individual markers would be greatly enhanced by their inclusion within a larger, well-integrated marking system.

Message redundancy would be increased, of course. But the additional cross-referencing and multiple linkages of markers, signs, symbols, text and diagrams also would help reduce the likelihood of inadvertent intrusion. Furthermore, use of teaching principles throughout the message system (i.e., defining the meaning of a given symbol or iconic sign by placement within appropriate linguistic and diagrammatic messages) would augment performance of the entire marker.

A central assumption is that future human beings from more, less and as advanced societies will be curious about the marker, and that some members will work actively to decode the monument's holistic design. Despite intelligent efforts, however, a monument designed by 20th-2Ist-century humans will present something of a mystery to future generations. We assume our descendants will respond to the challenge as eagerly as 20thcentury men and women have responded to questions and enigmas posed by ancient monuments.

The fact that people living in the 21st century made an effort to transmit a warning message to future generations would itself become a message, whether or not the marker system worked as efficiently as its designers had hoped. The effort itself, in other words, as clumsy as the design might be or seem to future generations, could still achieve the desired effect: a lowered probability of inadvertent human intrusion into the WIPP.

Part of the message to future societies, clearly, would be the 21st century's perceived level of effort in marking the site. A monumental, intellectually stimulating system would enhance performance with higher probability than would a less energetic design based on minimal investment, thought and creativity.

An oral tradition tethered to the marker system could emphasize (1) that it was designed to be the world's longest lasting human artifact, (2) that it was intended as a gift to guard the health of future generations, and (3) that it is the world's largest celestial "clock" marking the millennia. Hyperbole and altruism are strong themes in the world's folk tales, songs and myths. 


\section{Recognition of Marker System}

Recognition of the proposed marker system would not be expected to depend on the technological level of hypothesized future civilizations.

More advanced, as advanced and less advanced societies would encounter signs expressly designed for ease of interpretation in any culture. Extensive indexing, cross-referencing and teaching principles utilized on-site in the marking system would enable intelligent Homo sapiens from any future society to understand the message.

More advanced societies would have the least difficulty decoding the proposed marker system. Twentieth-century scientists have done an admirable job recognizing and interpreting ancient pictographs, symbols and archaic texts. Future, more advanced scientists would have fewer problems interpreting pictographs, symbols and scripts purposely designed for transparency of interpretation.

As advanced societies would be likely to share 21st-century assumptions and world view. A high probability exists that shared understandings would aid in future efforts to explain the markers.

Less advanced societies would not grasp subtleties encoded in the periodic table of the elements, perhaps, but would likely understand pictographic narratives and linguistic scripts. Future cultures unable to read any of the inscribed, written messages would be unlikely candidates for intrusion because of a lack of technical capabilities to do so.

The proposed marker system, therefore, would be designed to work for future societies in which technological knowledge increases, stabilizes or decreases.

Political Change. Regarding altered political control of the WIPP site, the above principles apply. Societal memory loss from a radical change in political control would be less likely to deter inadvertent intrusion than would conscious decisions by a new government to destroy all traces (especially monuments) of the old regime. Because material representations of culture reflect basic assumptions and foreign world-views, challengers might yield to destructive impulses. Use of multicultural (as opposed to parochial or nationalistic) messages, could, along with employment of culturally diverse symbols, languages and scripts, mitigate effects of altered political control across the 10,000 year span. 
Mescalero Apache Symbolism. To further enhance the transmission of the warning message, consideration should be given to including at least one Native American language among those chosen for the inscriptions, as well as the use of Native American symbolism on the markers. Only during the last three centuries have Spanish and English-speaking peoples been dominant in the plains east of the Pecos River, where the WIPP site is located.

For thousands of years Native American hunters and gatherers ranged across these plains. Apache people entered New Mexico from the north around $1400 \mathrm{~A} . \mathrm{D}$. and then worked their way south to the eastern plains (Opler, 1983; Pasztor, 1991). The group now known as the Mescalero Apache lived in the plains east of the Pecos from at least the 17th until the mid-19th century, when they were placed on a reservation just west of the Pecos.

The historic association of this Native American tribe with the region where the WIPP is located could be recognized by including Mescalero language and the symbolism drawn from their rich tradition of ethnoastronomy. Mescalero Apache itself is part of a widespread Indian language family, known as Athabascan, which includes other Apache languages of New Mexico and Arizona, along with Navajo languages spoken as far north as Canada and Alaska. A written message could be inscribed using the Roman alphabet's standard orthography, special Apachean characters and diacritical marks for tone agreed upon in 1975 by the Mescalero Apache Language Commission (Farrer, 1991, p. 262).

Like many Native American cultures, the Mescalero Apache "lived in the sky." That is to say, they responded with cultural sensitivity to motions of celestial bodies (Farrer, 1991; Williamson, 1984, pp. 289-319). Their "cosmovision," represented by the quartered circle (Figures 18 and 19), which still symbolizes the cardinal points, the course of the sun and Iife's circularity (Farrer, 1991; Farrer and second, 1981), could be used in the symbolism of the WIPP markers. According to Farrer (1991, p. 143): "The Creator gave the Mescalero Apache people serious responsibility for the maintenance of balance and harmony in the universe. Despite incursions of every imaginable sort by the larger mainstream Anglo culture, Apaches have persisted and maintained their responsibilities."

Vandals. The greatest threat to any unpoliced marker system would be vandalism. Destruction of markers by juvenile members of Homo (or soldiers, religious fanatics or political 

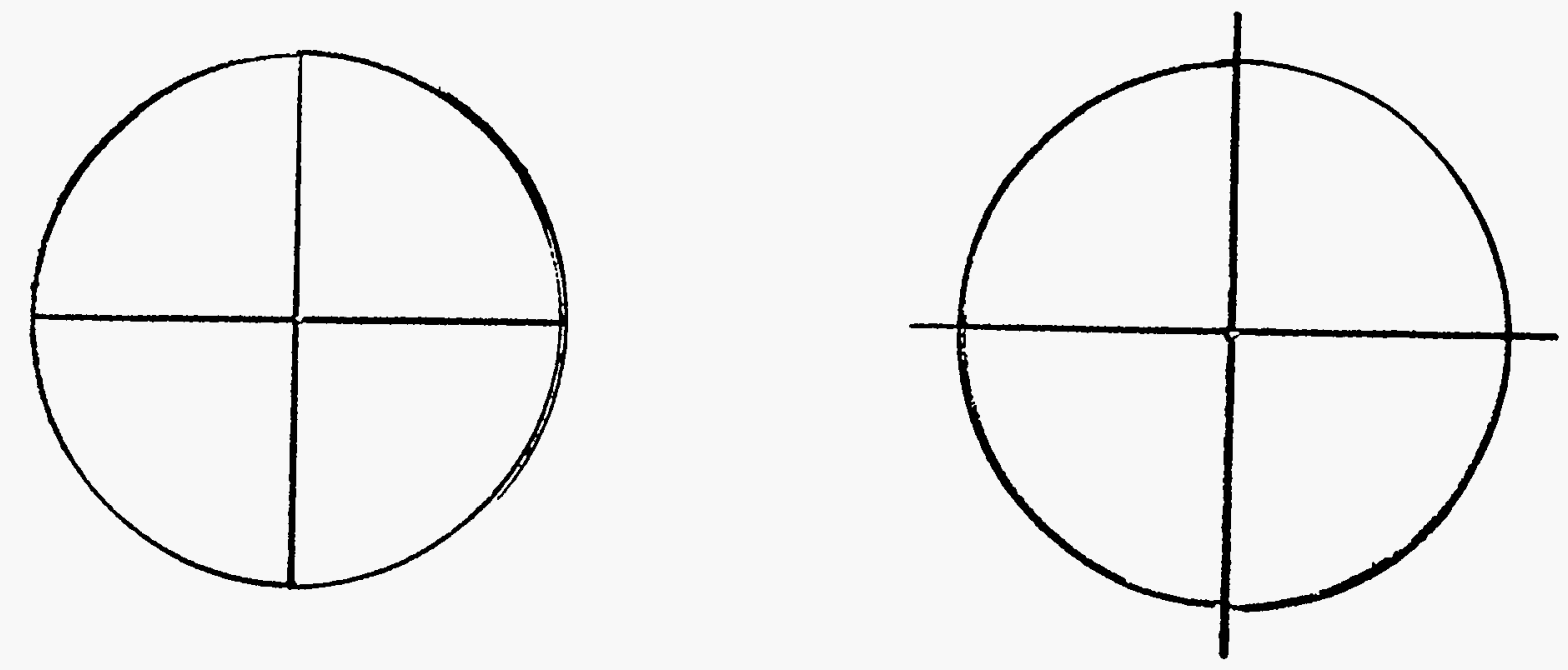

THE QUARTERED CIRCLE: VISUAL REPRESENTATIONS OF THE MESCALERO APACHE "BASE METAPHOR" (FROM CLAIRE FARRER, LIVING LIFE'S CIRCLE: MESCALERO APACHE COSMOVISION. UNIVERSITY OF NEW MEXICO PRESS, ALBUQUERQUE, 1991:96)

Figure 18. The Quartered Circle: Visual Representations of the Mescalero Apache "Base Metaphor" (Farrer, 1991). 


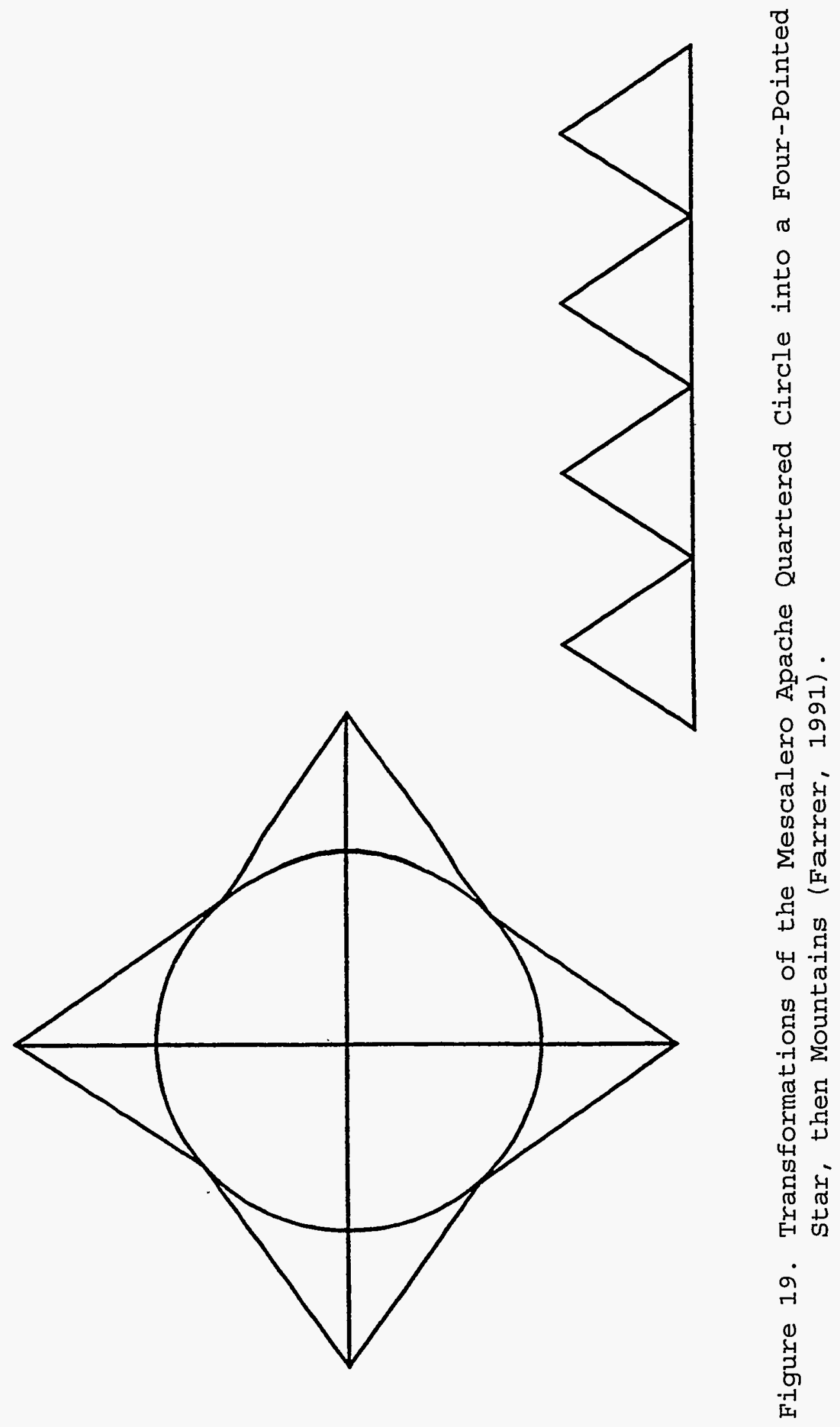


true-believers) could present the greatest challenge to efforts detailed in the present report. Redundancy would make total annihilation of the site by vandals impossible; yet the cumulative damage inflicted over 10,000 years by vandals from more advanced, as advanced and less advanced societies could greatly reduce the expected level of deterrence.

One defense would be to design a site that future generations considered valuable enough to preserve for their future generations. The better the monument, the more likely it would be protected-possibly even repaired-across 10,000 years.

Radical Increase in Consumption of World Resources. Continued population growth and a significant increase in resource consumption presents a scenario in which societies 2,000-10,000 years from now increasingly would be tempted to make advertent intrusions into the WIPP. Efficacy of the proposed warning system would not be expected to vary with population, extractive activity or resource demand. However, such demands would likely stimulate decisions to test the limits of the WIPP's warning message. But strictly speaking, intrusions based on knowledge would be advertent.

Radical Discontinuity. A major war or societal catastrophe would result in conditions similar to those detailed above for political change. Again, the proposed warning system would be targeted to more advanced, less advanced and as advanced literate societies. Its message would be designed for accurate interpretation across 10,000 years for people of all technological levels. Resource prices, economic disruptions and population growth would be unlikely to influence readability, but might be expected to inspire deliberate, advertent intrusions by excavation, tunneling and drilling, or by additional nuclear-waste storage efforts at the WIPP. 


\section{References Cited}

Costa, J.E., and V.R. Baker. 1981. Surficial Geology: Building with the Earth. New York, NY: John Wiley and Sons.

Desantillana, G., and H. Von Dechend. 1977. Hamlet's Mill: An Essay on Myth and the Frame of Time. Boston, MA: David R. Godine Pub., Inc.

Deeson, A.F.I., ed. 1973. The Collector's Encyclopedia of Rocks and Minerals. New York, NY: Clarkson N. Potter, Inc.

Evans, I.O. 1972. Rocks, Minerals and Gemstones. London, England: Hamlyn Publishing Group Ltd.

Farrer, C.R. 1991. Living Life's Circle: Mescalero Apache Cosmovision. Albuquerque, NM: University of New Mexico Press.

Farrer, C.R., and B. Second. 1981. "Living the Sky: Aspects of Mescalero Apache Ethnoastronomy," Archaeoastronomy in the Americas. Ed. R.A. Williamson. Ballena Press Anthropological Papers 22. Los Altos, CA: Ballena Press. 137-150.

Ford, J.P., R.G. Blom, J.A. Crisp, C. Elachi, T.G. Farr, R.S. Saunders, E.E. Theilig, S.D. Wall, and S.B. Yewell. 1989. Spaceborne Radar Observations: A Guide for Magellan Radar-Image Analysis. JPL-Publ-89-41. Pasadena, CA: Jet Propulsion Laboratory, California Institute of Technology.

Givens, D.B. 1982. "From Here to Eternity: Communicating with the Distant Future," Et Cetera. Vol. 39, no. 2, 159-179.

Greeley, R., and J.D. Iversen. 1985. Wind as a Geological Process: On Earth, Mars, Venus, and Titan. New York, NY: Cambridge University Press.

Hawkins, G.S. 1973. Beyond Stonehenge. New York, NY: Harper \& Row.

Henderson, F.M. 1985. "Active Microwave Imaging Systems," The Surveillant Science: Remote Sensing of the Environment. Ed. R.K. Holz. 2nd ed. New York, NY: John Wiley \& Sons. [234]-247.

Hogben, L.T. 1949. From Cave Painting to Comic Strip: A Kaleidoscope of Human Communication. New York, NY: Chanticleer Press. 
Isherwood, D., and A. Street. 1976. "Biotite-Induced Grussification of the Boulder Creek Granodiorite, Boulder County, Colorado," Geological Society of America Bulletin. Vol. 87, no. $3,366-370$.

Jastrow, R., and M.H. Thompson. 1977. Astronomy: Fundamentals and Frontiers. 3rd ed. New York, NY: John Wiley and Sons.

Krupp, E.C. 1983. Echoes of the Ancient Skies: The Astronomy of Lost Civilizations. New York, NY: Harper \& Row.

Kyselka, W., and R.E. Lanterman. 1976. North star to Southern Cross. Honolulu, HI: University Press of Hawaii.

Le Mouel, J-F. 1991. "Prehistory of North America," Prehistory: The World of Early Man. Ed. J. Guilaine. New York, NY: Facts on File. 109-122.

MacDonald, H.C., and W.P. Waite. 1973. "Imaging Radars Provide Terrain Texture and Roughness Parameters in Semi-Arid Environments," Modern Geology. Vol. 4, no. 2, 145-158.

Neurath, O. 1936. International Picture Language: The First Rules of Isotype. London, England: Kegan Paul, Trench, Trubner $\&$ CO.

Ogden, C.K. 1934. The System of Basic English. New York, NY: Harcourt, Brace \& Co.

Opler, M.E. 1983. "Mescalero Apache," Handbook of North American Indians. Volume 10: Southwest. Ed. A. Ortiz. Washington, DC: Smithsonian Institution. 419-439.

Pasztor, S.B. 1991. "IX. A Historical Perspective of Cultural Development in Southeastern New Mexico," Background Information Presented to the Expert Panel on Inadvertent Human Intrusion into the Waste Isolation Pilot Plant. Eds. R.V. Guzowski and M.M. Gruebel. SAND91-0928. Albuquerque, NM: Sandia National Laboratories. IX-1 through IX-38.

Pettengill, G.H., P.G. Ford, W.T.K. Johnson, R.K. Raney, and L.A. Soderblom. 1991. "Magellan: Radar Performance and Data Products," Science. Vol. 252, no. 5003, 260-265.

Sabins, F.F., Jr. 1978. Remote Sensing: Principles and Interpretation. San Francisco, CA: W.H. Freeman. 
Singh, P. 1978. Stone Observatories in India, Erected by Maharaja Sawai Jai Singh of Jaipur, 1686-1743 A.D., at Delhi, Jaipur, Ujjain, Varanasi, Mathura. 1st ed. Varanasi: Bharata Manisha.

Singh, P. 1986. Jantar-Mantars of India: Store Observatories: Jaipur, Delhi, Ujjain, Varanasi, Mathura. Jaipur, India: Holiday Publications.

Smith, D.G., ed. 1981. The Cambridge Encyclopedia of Earth Sciences. New York, NY: Crown Publishers, Inc. and the Press Syndicate of the University of Cambridge.

Stover, L.E., and B. Kraig. 1978. Stonehenge: The Indo-European Heritage. Chicago, IL: Nelson-Hall.

Swadesh, M. 1952. "Lexico-Statistic"Dating of Prehistoric Ethnic Contacts," Proceedings of the American Philosophical society. Vol. 96, no. 4, 452-463.

Twidale, C.R. 1982. Granite Landforms. New York, NY: Elsevier Scientific Publishing Company.

Ulansey, D. 1989. The Origins of the Mithraic Mysteries: Cosmology and Salvation in the Ancient World. New York, NY: oxford University Press.

Williamson, R.A. 1984. Living in the Sky: The Cosmos of the American Indian. Boston, MA: Houghton Mifflin.

Winkler, E.M. 1975. Stone: Properties, Durability in Man's Environment. 2nd rev. ed. New York, NY: Springer-Verlag.

Worthen, T.D. 1991. The Myth of Replacement: Stars, Gods, and Order in the Universe. Tucson, AZ: The University of Arizona Press. 


\section{APPENDIX A \\ TESTING MARKER SYSTEMS FOR UNDERSTANDABILITY}

by Louis Narens

The ideas for candidate marker systems should be thoroughly tested for understandability by various target populations before a selection of the final system is made. It is very likely that due to the novelty and nature of the project, much testing and refining candidate marker systems will be required. Some aspects of the testing can be successfully achieved in a modular way (i.e., various messages or pieces of messages can be tested independently of others), while other aspects may need to test a mockup of the entire system to succeed.

This Appendix presents some testing concerns about marker systems similar to the kind of system that Team $B$ has proposed.

In Team B's system, there are three different kinds of explicit messages and a number of implicit ones. Each kind of explicit message makes different assumptions about the knowledge and capabilities of the reader and the kind of society to which he or she belongs. The rationale for testing of these kinds of messages will be based in part on these assumptions.

The SCIENTIFIC MESSAGE assumes a reader with scientific sophistication-particularly an understanding of physical chemistry. In the present world all potential readers of such a message have essentially the same cultural understanding of physical chemistry (e.g., their coursework and textbooks, although possibly of different traditions and languages, share the same concepts, experimental and mathematical methods, facts, and roughly the same kind of quality judgments of what is important and what is good work). Of course, we do not assume that future physical chemists will necessarily have cultural understandings similar to the current one. Thus, given this limitation of culturally diverse populations for testing, the best we can expect of testing for this case is to verify that the scientific message is easily understandable to members of the scientific community with knowledge of physical chemistry, and establish the level of expertise and intelligence needed for correctly interpreting the message.

The WRITTEN MESSAGE assumes a reader who is familiar with a portion of a variant of at least one of the languages the message is in. (For the purpose of exposition we will assume that the message is written in English) The written messages should be tested for understanding on people with a limited understanding of English, e.g., people who have English as second or third 
language, people who speak an English form of Pidgin. It is especially important to test the written message on people who are from cultures very different from our own, including nontechnological ones.

The (NONSCIENTIFIC) PICTOGRAPHIC MESSAGE assumes moderately intelligent reader or group of readers who is willing to put in the time and effort to "decode" it. This message should be tested in even a wider variety of cultures than the previous messages, especially ones that have very limited understanding of our culture and technology.

We assume that the nonscientific pictographic message will require much prior testing and redesigning. Much of this testing can be modular and can be somewhat generic in the sense that many of the results will be applicable to forms of the message. For example, variants of a symbol (or subset of symbols) can be tested to see which is most understandable; or various schemes for "teaching" a meaning of a symbol can be tested, etc. Both the resulting good symbols and good teaching methods can be then used as a basis for a variety of pictographic messages.

The creators of various candidate marker systems will have ideas about how the overall design of the markers, the graphic forms of the messages, as well as the messages themselves will likely inform potential readers about the designers of the messages, the nature of the society they came from, and possible reasons for the marker system, etc. Since it is very likely that potential readers will use ideas (perhaps preconceived ones) about the designers of the markers, their society, and reasons for the markers) as an initial basis for deciding how to go about interpreting the messages, it is important that the designers' ideas about such issues be made explicit and be tested. Also because of this, it is imperative that the messages themselves also be tested in ways that simulate contexts in which readers might encounter them. This may mean building a mockup of the Markers Project in some nontechnological culture and having people of that culture explain what it is about, or sending drawings and information about the site to various types of specialists of various cultural backgrounds for their opinions, etc.

In view of the above suggestions for testing marker systems, the following four considerations should be stressed:

1. The results of testing should feed back into the designing process. 
2. Consideration of culture plays an important role in the testing, and thus anthropologists should be strongly involved in the entire testing process.

3. A variety of kinds of individuals and cultures should be used in the testing process; and in particular, nontechnological cultures who have limited contact with our culture should be included.

4. The designers of the messages should make explicit the means they think various possible readers of the message will use to decode the appropriate message, and it should be tested whether or not various target populations use these means or others in reaching their conclusions.

5. The proper testing of a candidate marker system, while not very expensive in terms of the actual construction of the marker system, may take several years to complete. 
Appendix G: Team B Report

APPENDIX B

SUPPLEMENTAL MATERIAL ON THE WIPP MARKER

by Jon Lomberg

P.O. Box 207

Honaunau, Hawaii, 96726

I. Use of Symbols in the WIPP Marker .................

II. . The WIPP Pictograph .......................

III. Marking by Direct Means: Using a sample of waste as part of the Marker system .....................

IV. Notes on Marker Aesthetics and Design ..............G-84

References ................................ . . . . 90 


\section{USE OF SYMBOLS IN THE WIPP MARKERS}

There are three basic messages that symbols associated with the WIPP marker might be used to convey:

POISON

\section{RADIOACTIVE MATERIALS}

\section{DON'T INTRUDE}

Most cultures use visual symbols, but they all use different symbols. Our team was unable to discover any "universal" visual symbols that are guaranteed to be understood by any human being in conveying any of these three messages.

Some existing symbols were suggested by various members of our team. These included:

1) RADIATION TREFOIL

2) SKULL AND CROSSBONES

3) MR. YUK (a recently adopted international poison warning symbol for children)

4) DO NOT (circle with slash)

5) DIAGONAL SLASH

6) $X$ (something crossed out)

7) STYLIZED URANIUM ATOM

We have used the radiation trefoil in our sample pictographs and other drawings submitted with this report, but we want to emphasize that this was only done as a matter of graphic convenience. Given limited time, the simple trefoil was more convenient to sketch quickly than the more complicated skull and crossbones or some group of many symbols (as is proposed below).

The radiation trefoil was the subject of some vigorous debate. Some argued that the trefoil had already been adopted internationally and might well survive for many centuries with its meaning more or less intact. Others were concerned that the trefoil is not iconographic, that is there is nothing about it that directly relates to the concept it symbolizes. One team member quipped, upon seeing the trefoil used to signify waste in our sample pictograph, "Why are they burying all those submarine propellers?" 
The skull and crossbones also received a good deal of consideration, including some external advice from Carl Sagan, who had written a letter to the project on the subject (see letter from Carl sagan at the end of this Appendix B). The lineage of the skull and crossbones as a graphic symbol (as opposed to the use of real bones in totems and "cannibal lintels") leads back to medieval alchemists, for whom the skull represented Adam's skull and the crossed bones the cross that promised resurrection. It is almost certainly a western cultural artifact, yet it too has spread worldwide as a symbol for poison -and also for pirates. Henry Dreyfuss, a great scholar of symbols, once performed an experiment where 3-year old children were shown a skull and crossbones and immediately shouted "Pirates". If they were shown the skull and crossbones positioned on a bottle, they shouted "Poison". It is for this reason that the skull and crossbones that appear in the drawings are sometimes shown set upon a bottle.

Some general principles were agreed upon:

1) No symbol is certain to stay in use for the 10,000 year period. Future societies will probably create many of their own symbols, and symbols from our time may have their meanings changed or distorted with the passage of time. Compare how the meaning of the swastika has changed in our own century, going from positive religious symbol of India to a hated emblem of the Nazis.

2) Symbols used in the WIPP Marker should be defined by pictographs as part of the marker. Some examples of how this might be done follow.

Figure 5 shows how the "Do Not" symbol might be defined. Figure 6 shows how the skull and crossbones could be shown to mean "poison" (though there should probably be an additional frame that shows the sick person as a skeleton). Figure 7 shows how difficult it is to define a radiation hazard symbol without requiring that the reader have a knowledge of chemistry or physics. The problem is that the effects of exposure to radiation can take many years to appear. In Figure 7 one possible solution to this is presented: A child encounters the waste (symbolized by the trefoil), and the symbol is transferred onto his chest. In the background are some young trees. Then the child is seen as an adult, identified with the child by the symbol on his chest. The tree is several decades older, but still recognizable by the pattern of its branches. Flowers at the base of the young and old tree provide a measure of scale. An additional frame might be added to this sequence showing the adult clearly dead. 
We do not suggest that any of these sequences are developed enough to use, but they perhaps point the direction in which other definitional pictographs could be developed.

3) Geometrically simple and symmetrical symbols (such as the trefoil or the "X") can withstand more degradation and later be reconstructed than can more complex symbols like the skull and crossbones. Two drawings show how a system of berms might be used to form a symbol visible from above (Figures 1 and 2).* Remote marking using materials of different dialectric, thermal, or magnetic properties could also be shaped in this way. The trefoil can be severely degraded by wind or water erosion, or by excavation, and still be recognized. As soon as the skull and crossbones begins to lose its definition, there will perhaps be those future observers who argue that we are merely projecting a face onto a random arrangement of material, as is almost certainly the case with the so-called Face on Mars.

4) Multiple symbols might be easier to read than single symbols. While experimenting with various symbols, this artist noticed that the symbols were sorting themselves into pairs as follows:

TREFOIL \& STYLIZED ATOM

CROSSBONES \& MR. YUK

SLASHED CIRCLE \& $\mathrm{X}$

If these symbols are grouped together as pairs, the sum of two half-understood symbols might be two fully understood symbols. For example, the trefoil itself might seem to some future readers like an old radiation sign, while others argued that it looked floral (a French ruin?) or like a Japanese mon (a clan crest). The stylized atom, if seen alone, might be mistaken for a solar system. But together, the two symbols help confirm the correct hypothesis and disprove the incorrect hypothesis. Boxing the symbols together is one way to enforce the sense of their connection. Figure 8 shows how groups of symbols might be designed to convey the three basic warning messages.

A detail that requires more thought is what to put behind the slashed circle or the $X$. A drilling tower such as the one shown may not be recognized for what it is. But a stylized stick figure of a person digging doesn't convey the danger correctly, since it is not dangerous to dig a few feet down; perhaps the more detailed information on the pictographic sequence showing the history of the WIPP will clarify that possible confusion.

\footnotetext{
*The drawings give a generalized view of how our Markers might appear: a ring of monoliths circumscribing the area directly over the waste, with a central structure that contains the most detailed information. The WIPP building, or some remaining portion of it, is due north of the central structure.
} 
A fourth pair of symbols might be added to help explain that the two symbols in each box are equivalent symbols of the same concept (see Figure 9).

5) Symbols could be used in association with the linguistic messages. Use of an identical symbol or group of symbols before each language could help suggest that each language is saying the same thing (see Figure 10).

6) Symbols can and should be tested to determine which symbols work best and how their presentation affects their ability to be understood: for example, does it make any difference if symbols are drawn as outlines or filled figures? (see Figures 11 and 12).

\section{II) THE WIPP PICTOGRAPH}

A sketch of the kind of pictorial narrative that might be used on the WIPP Marker.

\section{BACKGROUND}

Not all human cultures have painting, drawing, or other graphic arts. In those that do, the human figure is a common subject. People and animals are the most easily recognized elements in the pictures of another culture. Symbolic elements, emblems of natural forces, decorative motifs, and scripts can be difficult to interpret in prehistoric cave murals, Egyptian frescoes, Chinese scrolls, Persian miniatures, or Plains Indians hide paintings. But the human figure is usually clear. Universal human actions-running, digging, paddling a boat, hunting-are also usually easy to recognize.

Often sequences of images are used to depict events in time in a linear fashion, to tell a story or record a historical event; there are many examples of linear, pictorial narratives in existence. Three independently evolved examples-the Bayeaux Tapestry (France, 12th Century); the Japanese scroll "The Mongol Invasions" (Japan, 13th Century); and the Lakota Sioux picture story of the Battle of the Little Big Horn (United states, 19th Century)-indicate how widespread the use of pictures to create narrative is. Drawing and reading comic strips is one the most ancient and widespread of human pastimes.

The human figure is the object likeliest to be recognized by those people of the future who find the WIPP Markers. The human figure provides a natural scale for determining the size of objects in the pictures, and helping recipients interpret and calibrate the numbers and measurements used. A linear narrative contained in panels, read from top to bottom, seems a good way of conveying the history of the site. 
TECHNIQUES

One difficulty facing an artist who wants to show human figures at the WIPP site is that the depth of the repository is such that to show the underground chambers scaled correctly, more than 2200' have to be contained in the frame. The human figure is far too small in any reasonably sized frame.

The size of the frame is constrained by the number of frames required to tell the story and the height of the marker. Top to bottom reading is done in all cultures. Horizontal readings provide more ambiguity, no matter how they are marked. The clearest way to present the narrative is in a single stack of frames, avoiding the confusion possible if readers have to go from the bottom of one column of frames to the top of another. Therefore the size of the frame can be determined by dividing the height of the easily readable area of the marker by the number of frames. Museum exhibit designers generally try to contain all text and diagrams between 3 feet and 6 feet from the floor. The sequence of drawings for Figure 13 has 10 frames. To fit 10 frames in 3 feet, each frame can be no higher than 3.6 inches. Actually, since frames require a little spacing, the actual height would be less than that.

But large frames can be seen even higher than 6 feet. A more generous estimate of the maximum reading area might be between $10^{\prime}$ and $2^{\prime}$. Now the height of each panel is over 9 inches. If the WIPP building and the repository are shown to scale in a frame of this size, then a $6^{\prime}$ person is less than one fortieth of an inch high. Just a featureless dot on the horizon.

The conventional solution used in western style comic strips is to cut to scenes at different scales, much as a movie director will cut from a long shot to a closeup. But this is not a convention that has been used universally, and it provides many ambiguities.

\section{THE STORY OF WIPP}

The solution I propose is to have two parallel stories presented in a stack of paired frames. One shows the WIPP site at large scale, the other at human scale. Deliberate efforts have been made to associate each frame in the pair by date, size scale, and stars in the sky. The hope is that future decoders will be able to figure out that they are seeing the entire site and a closeup and various moments in time. Tally dots beneath each frame reinforce the sequencing-and perhaps the sense of time passing-implied by the sequence of frames. 
The long shot frames show the desert as it was before WIPP, oriented by the North star, the appearance of WIPP, the sinking of the shafts and the excavation of the chambers, the transportation of waste material to the site, down the shaft, and into the chambers, the sealing of the shaft, the erection of the markers, the change in the Polar constellations due to the Earth's precessional cycle, and the decay and disappearance of the buried hazard.

The close-up frames cut from surface activities at the site to activities beneath the surface. The absence of stars in the sky and the indicated depth should be $a$ clue as to the difference. Even if recipients cannot actually read the depths, they should be able to recognize that the "2150" is associated with some of the close-ups and that $0^{\prime}$ is associated with others. Comparing these two numbers with their positions in the long shot will help decode the location of the scenes.

NOTE: The Trefoil has been used as a symbol for the waste matter purely as a matter of the artist's convenience: it was fast for me to draw in. The actual symbol or group of symbols that should be used on the marker is a matter requiring a great deal of further study and testing.

III) MARKING BY DIRECT MEANS: Using a sample of waste as part of the Marker system.

No symbolic representation of the radioactive material could be as unambiguous as the material itself. A properly sealed, transparent canister (Figure 20) containing a representative sample of the gloves, glassware, and sludge buried at WIPP would be harmless, indeed unobserved, by the casual visitor. Sealed containers containing small amounts of waste could be buried at strategic spots in the Marker area e.g. under the central structure, beneath the shaft seals, or with the other buried monoliths. Perhaps the "remote" marker in Carlsbad Caverns would be another possible site for a waste sample.

A sample of waste might be the most effective way of deterring -or at least slowing down-the potential intruder who was serious enough to have begun excavation. The sample could be marked with the symbols representing the buried material, and thus provide the best possible proof of the meaning of the symbols used elsewhere on the site. The discovery of the waste sample might help steer intruders away from any hypothesis that nuclear weapons, weapons grade materials, or extractable ores were contained in the repository. The contents of WIPP are so uninspiring and worthless that a sample might be the best means 


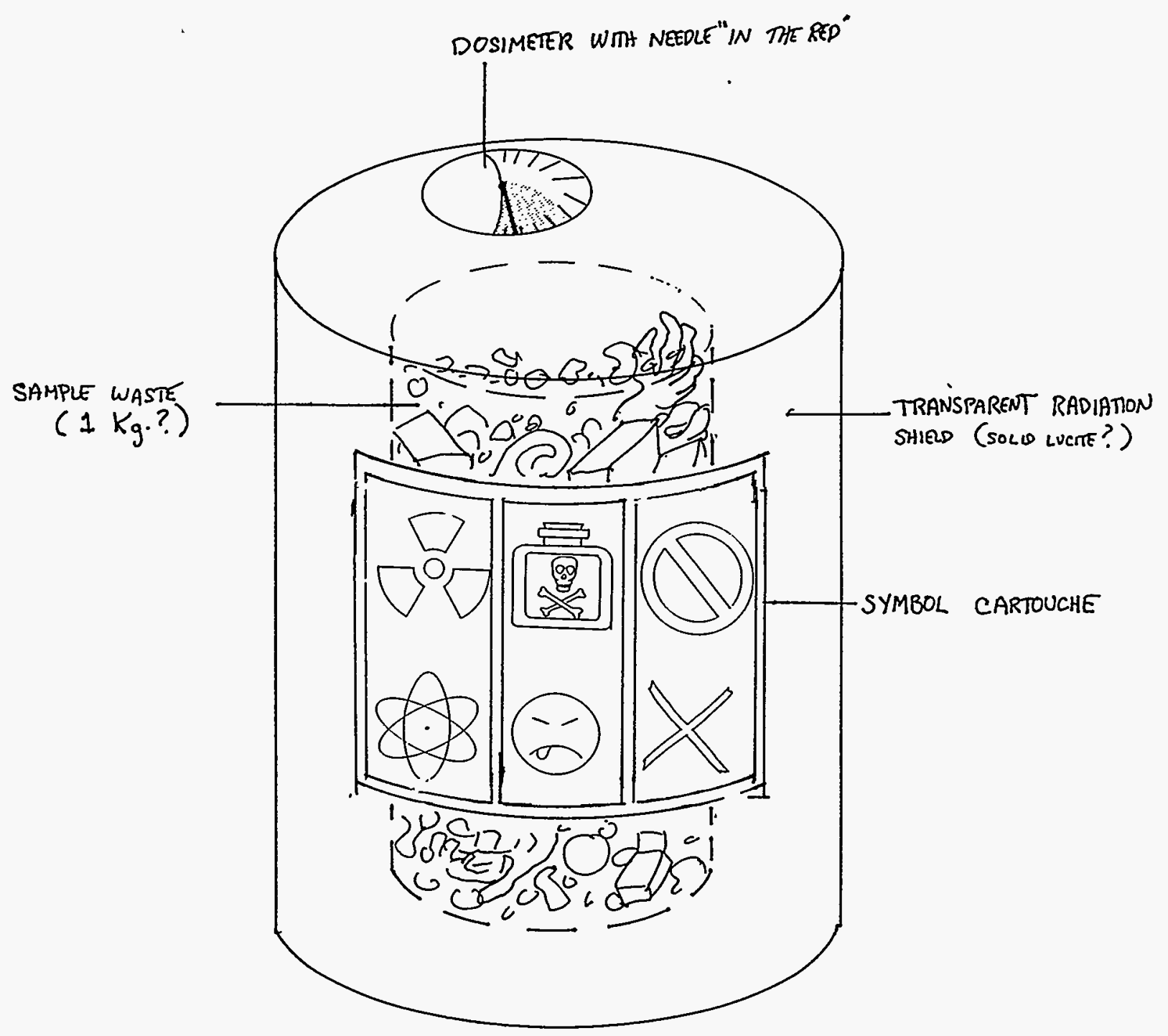

ACCESSIBLE bURIED SAMPLE in PROTECTIVE CONTAINER

Figure 20. Transparent Canister of Sample WIPP Waste (art by Jon Lomberg). 
of deterring intruders from undertaking the difficult and costly task of re-opening the chambers.

\section{IV) NOTES ON MARKER AESTHETICS AND DESIGN}

Various members of the Marker Panel have expressed the view that the Marker should be designed so as to achieve maximum aesthetic impact, so as to be seen as a "gift from our century to the future" (Givens), involving contemporary artists working on large scale environmental sculpture (Sullivan), or using Jungian archetypal forms to create a mood of dread and danger (Brill).

As a professional artist, I wish to register a dissenting view. I believe that the Marker should be designed purely on functional grounds, and that any attempts to make the Marker some kind of artistic statement are bound to confuse the clarity of the basic message we are trying to convey. There are several reasons for this belief:

\section{ART IS AMBIGUOUS.}

Art is sometimes described as a universal language. Some aspects of art can help bridge gaps when there is no common verbal language, and that is the basis of the idea of using a pictographic sequence to convey some aspects of the nature of the WIPP site. But art can as often be the most ambiguous form of human communication, especially when we are trying to understand the intent of the artist. For example, the depictions of the animals in cave paintings are easily understood, but it is much harder to determine why the paintings were created. Representational art is much more easily understood than symbolic art, and the direction that most artists take in large scale sculpture is symbolic or abstract. I believe that most of the designs that would be suggested by sculptors or "Earth Artists" would be more abstract than representational. Contemporary audiences often voice puzzlement over the intent of abstract painters and sculptors. Any inclusion of abstract, geometrical, or symbolic forms in the Marker is more likely to confuse than to enhance the meaning of the Marker.

2. ART IS AN END IN ITSELF.

Even if we could commission some monument great enough to become a wonder of the world whose fame would be carried down 300 generations, the very fact that the Marker was so impressive could lead to the belief that the purpose of the marker is artistic rather than communicative. A large and powerful sculpture sitting in the middle of the desert could easily be seen as the product of some individual artist-similar to Mt. 
Rushmore or current endeavors like Charles Ross' "Star Axis" (also under construction in New Mexico) rather than as an organized attempt at communication. Some of these large-scale artworks may also survive millennia. The WIPP Marker shouldn't be mistaken for another example-however well designed-of a 20 th Century school of outdoor sculpture.

Art usually has no function, it exists only to be experienced. If people of the future view the WIPP Marker as a piece of art, they are less likely to try to interpret it as conveying a particular message rather than as some elaborate "artistic statement".

These comments apply equally well to art which intentionally tries to be ugly or convey a mood of dread or danger, as in some of Mike Brill's (A Team) imaginative designs. Not all great art is meant to be beautiful. Consider Picasso's painting "Guernica" in this context. Sculptural forms which convey a negative emotion or mood may also be seen as "merely" works of art, with no explicit marking function.

\section{ART DRAWS PEOPLE TO IT.}

We want people to stay away from this site, not travel from distant places to see it. A great and famous work of art encourages visits from other artists, historians, and tourists. If enough people want to come to see a remote wonder, somebody will put up a hotel to accommodate them. Maybe the hotel decides to drill for water... By creating a great monument we may be causing the developments at the site that we most want to avoid.

\section{GREAT ART IS HARD TO COMMISSION.}

For every successful commissioned monument there are a hundred failures, e.g., the Prince Albert memorial in Iondon (an architectural laughingstock) or the WWII Airman's Memorial in Toronto (known locally as "Gumby Goes to Heaven"). If a decision is made to have a competition for a sculpture, a momentum is established whereby one piece has to be selected, whether or not somebody has come up with the right design.

I am also very concerned about who would decide which design would be used. Let me remind the Panel that the thinking that now dominates the art world in places like New York is antiscientific, anti-representational, and seems to favor more detached and (to me) nihilistic statements of artists. I do not think that the art community as it exists would be well qualified to create or select a design that would be scientifically informed about the many intricacies of this problem (encroachment by sand dunes, durability of materials, future scenarios, etc.) 
Yet if an announcement were made that there was going to be a grand competition for a Marker to last 10,000 years, it would be hard not to involve the art community in the decision making process. If you do, be warned: they are likely to end up picking a giant inflatable hamburger to mark the site.

I say let artists submit designs if they wish, but don't decide a priori that any design will in fact be used.

5. AN ARTISTIC MARKER MAKES INTERNATIONAL STANDARDIZATION OF MARKERS LESS LIKELY.

We all seem to agree that having similar markers appear worldwide at other nuclear waste repositories is a good idea. A purely functional narker, if well conceived and not too site specific has a chance of being adopted internationally. But as soon as you make it an artistic competition, you invite nationalistic competitiveness. I cannot believe, for example, that France would want a sculpture selected by an American committee to grace a French repository. And if each country wants an artistic statement that reflects their own (contemporary) artistic beliefs and styles, the Markers will very quickly diverge, making it harder for people of the future to realize that all these sites have some common link.

For all of the above reasons, I urge that the Marker be designed purely on the criterion of message clarity.

An example of a marker designed purely for function is shown in Figure 3. The design was inspired by the placement of an Indian pictograph painted in the year 1054 A.D. which recorded the appearance of a supernova in the sky. The painting was done on the underside of an overhang, perhaps the best location to minimize the effects of rain and windblown dust. Different kinds of information could be placed on different faces of the marker. For example the symbols and languages could be placed on the side facing outward, information about the site's relation to the Earth's precessional cycle could be placed on the side facing inward. Variations on this design might have niches or other recesses carved into each monolith in which the most important information could be engraved.

According to the present dimensions stipulated as those of the actual waste storage area, it is possible to calculate how large a 30 foot monolith on the perimeter of a ring shown in Figure 2 would appear from the center Figure 21. 


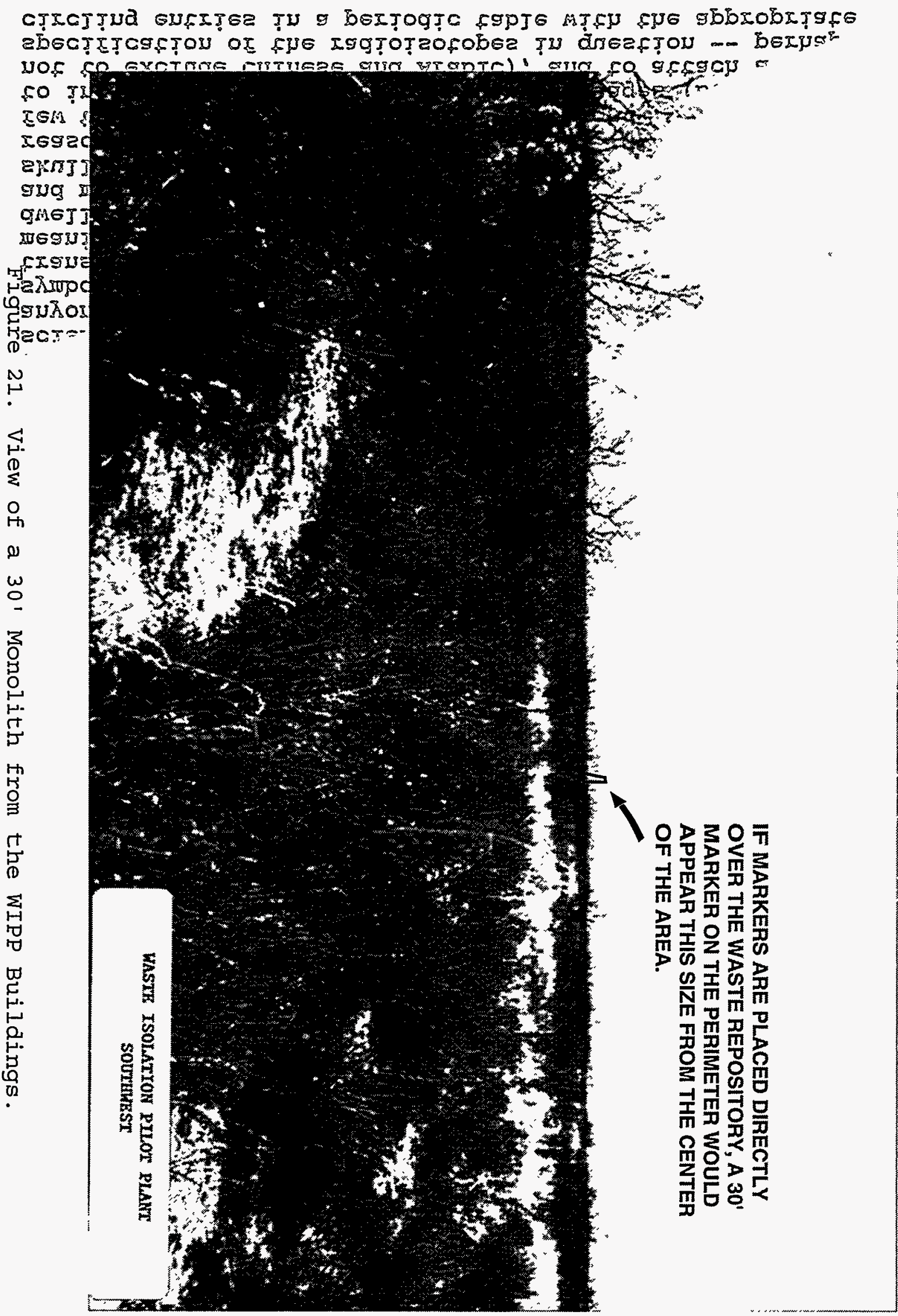


Appendix G: Team B Report

\section{CORNELL UNIVERSITY \\ Center for Radiopbysics and Space Research AUG 171990 \\ SPACE ScIences BuIIDING \\ Ithaca, New York 14853.6801}

Telephone (607) 255.4971

Laboratory for Planetary Studies

Fax (607) 255.9888

8 August 1990

Dr. D. Richard Anderson

Performance Assessment

Division 6342

Sandia National Laboratories

Albuquerque, New Mexico 87185

Dear Dr. Anderson:

Many thanks for your kind invitation to participate in the panel charged with making recommendations on signing to the far future about the presence of dangerous long-lived radioactive waste repositories (assuming the waste hasn't all leached out by then). It is an interesting and important problem, and I'm sorry that my schedule will not permit me to participate. But I can, in a few sentences, tell you my views on the matter: perhaps you would be kind enough to pass them on to the members of the panel:

Several half-lives of the longest-lived radioisotopes in question constitute a time period longer than recorded human history. No one knows what changes that span of time will bring. Social institutions, artistic conventions, written and spoken language, scientific knowledge and even the dedication to reason and truth might, for all we know, change drastically. What we need is a symbol invariant to all those possible changes. Moreover, we want a symbol that will be understandable not just to the most educated and scientifically literate members of the population, but to anyone who might come upon this repository. There is one such symbol. It is tried and true. It has been used transculturaliy for thousands of years, with unmistakable meaning. It is the symbol used on the lintels of cannibal dwellings, the flags of pirates, the insignia of ss divisions and motorcycle gangs, the labels of bottles of poisons -- the skull and crossbones. Human skeletal anatomy, we can be reasonably sure, will not unrecognizably change in the next few tens of thousands of years. You might very well wish also to include warnings in major human languages (being careful not to exclude (hinese and Arabic), and to attach a specification of the radioisotopes in question -- perhaps by circling entries in a periodic table with the appropriate 
Dr. D. Richard Anderson

8 August 1990

page 2

isotopic atomic numbers emphasized. It might be useful to include on the signs their own radioactive markers so that the epoch of radioactive waste burial can be calculated (or maybe a sequence of drawings of the Big Dipper moving around the Pole star each year so that, through the precession of the equinoxes, the epoch of burial, modulo 26,000 years, could be specified). But all this presumes much about future generations. The key is the skull and crossbones.

Unless a more powerful and more direct symbol can be devised, I think the only reason for not using the skull and crossbones is that we believe the current political cost of speaking plainly about deadly radioactive waste is worth more than the well-being of future generations.

With best wishes,

Cordially,

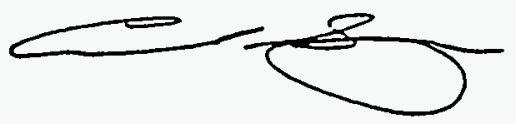

Carl Sagan

cc: Jon Lomberg

Ann Druyan 


\section{REFERENCES}

Brodrick, A.H. 1948. Prehistoric Painting. London, England: Avalon Press Ltd.

Dreyfuss, H. 1972. Symbol Sourcebook: An Authoritative Guide to International Graphic Symbols. New York, NY: McGraw-Hill.

The Herdex Symbol Dictionary. 1986. Wilmette, IL: Chiron Publications.

Johnson, F.C., L.A. Johnson, and G. Dykstra. 1971. Stick Figure Drawing For Language Teachers. London, England: Ginn.

Kidder, J.E. 1981. The Art of Japan. New York, NY: Park Lane. Lehner, E. 1950. Symbols, Signs, and signets. Cleveland, OH: World Publishing Co.

Sagan, C., F.D. Drake, J. Lomberg, I.S. Sagan, A. Druyan, and T. Ferris. 1978. Murmurs of Earth: The Voyager Interstellar Record. New York, NY: Random House.

Soisson, P., and J. Soisson. 1978. Life of the Aztecs in Ancient Mexico. Barcelona, Spain: Productions Liber. 


\section{DISTRIBUTION}

(Send Distribution list changes to M.M. Gruebel, Dept. 6342, Sandia

National Laboratories, PO Box 5800, Albuquerque, NM 87185-1328)

\section{Federal Agencies}

US Department of Energy (6)

Office of Civilian Radioactive Waste Management

Attn: Deputy Director, RW-2

Associate Director, RW-10/50 Office of Program and Resources Management Office of Contract Business Management

Director, RW-22, Analysis and Verification Division Associate Director, RW-30 Office of Systems and Compliance

Associate Director, RW- 40 Office of Storage and Transportation

Director, RW-4/5 Office of Strategic Planning and International Programs Office of External Relations

Forrestal Building

Washington, DC 20585

US Department of Energy

Albuquerque Operations office

Attn: National Atomic Museum Library PO Box 5400

Albuquerque, NM 87185

US Department of Energy (2)

Office of Environmental Restoration and Waste Management

Attn: Director, EM-1

C. Frank, EM-50

Washington, DC 20585

US Department of Energy (3)

Office of Environmental Restoration and Waste Management

Attn: M. Frei, EM-34 / Trevion II

Director, Waste Management Projects Washington, DC 20585-0002

US Department of Energy

Office of Environmental Restoration and Waste Management

Attn: J. Lytle, EM-30 / Trevion II

Washington, DC 20585-0002
US Department of Energy

Office of Environmental Restoration

and Waste Management

Attn: S. Schneider, EM-342

Trevion II

Washington, DC 20585-0002

US Department of Energy (3)

WIPP Task Force

Attn: G.H. Daly

S. Fucigna

B. Bower

12800 Middlebrook Rd., Suite 400

Germantown, MD 20874

US Department of Energy (4)

Office of Environment, Safety and Health

Attn: R.P. Berube, EH-20

C. Borgstrum, EH -25

R. Pelletier, EH-231

K. Taimi, EH-232

Washington, DC 20585

US Department of Energy (6)

WIPP Project Integration Office

Attn: S. Alcorn

W.J. Arthur III

J. Coffey

L.W. Gage

P.J. Higgins

D.A. Olona

PO Box 5400

Albuquerque, NM 87115-5400

US Department of Energy (2)

WIPP Project Integration Satellite

Office

Attn: R. Batra

R. Becker

PO Box 3090, Mail Stop 525

Carlsbad, NM 88221-3090

US Department of Energy (10)

WIPP Project Site Office (Carlsbad)

Attn: A. Hunt (4)

V. Daub (4)

$\mathrm{J}$. Lippis

$\mathrm{K}$. Hunter

PO Box 3090

Carlsbad, NM 88221-3090 
US Department of Energy

Research \& Waste Management Division

Attn: Director

PO Box $E$

Oak Ridge, TN 37831

US Department of Energy (2)

Idaho Operations Office

Fuel Processing and Waste

Management Division

785 DOE Place

Idaho Falls, ID 83402

US Department of Energy

Savannah River Operations Office

Defense Waste Processing

Facility Project Office

Attn: W.D. Pearson

PO Box A

Aiken, SC 29802

US Department of Energy

Richland Field Office

Attn: R.F. Guercia

PO Box 550 / R3-80

Richland, WA 99352

US Department of Energy

Office of Geologic Disposal

Yucca Mountain Project Office

Attn: Associate Director, RW-20

PO Box 98608

Las Vegas, NV 89193-8608

US Department of Energy (3)

Nevada Operations office

Attn: J.R. Boland

D. Livingston

P.K. Fitzsimmons

2753 S. Highland Drive

Las Vegas, NV 89183-8518

US Department of Energy (2)

Technical Information Center

PO Box 62

Oak Ridge, TN 37831

US Department of Energy (2)

Chicago Operations Office

Attn: J.C. Haugen

9800 South Cass Avenue

Argonne, IL 60439
US Department of Energy (3)

Rocky Flats Area Office

Attn: W.C. Rask

G. Huffman

T. Lukow

Po Box 928

Golden, CO 80402-0928

US Department of Energy

Los Alamos Area Office

528 35th Street

Los Alamos, NM 87544

US Department of Energy

Dayton Area Office

Attn: R. Grandfield

PO Box 66

Miamisburg, OH 45343-0066

US Bureau of Land Management

Carlsbad Office

101 E. Mermod

Carlsbad, NM 88220

US Bureau of Land Management

New Mexico State Office

PO Box 1449

Santa $\mathrm{Fe}$, NM 87507

US Environmental Protection

Agency (2)

Radiation Protection Programs

Attn: M. Oge

ANR -460

Washington, DC 20460

US Environmental Protection

Agency, Region 6

Attn: C. Byrum, 6T-ET

1445 Ross Ave.

Dallas, TX 75202

US Geological Survey (2)

Water Resources Division

Attn: C. Peters

4501 Indian School NE

Suite 200

Albuquerque, NM 87110

US Nuclear Regulatory Commission

Division of Waste Management

Attn: H. Marson

Mail Stop 4-H-3

Washington, DC 20555 
US Nuclear Regulatory Commission (4) Advisory Committee on Nuclear Waste

Attn: D. Moeller

M.J. Steindler

P.W. Pomeroy

W.J. Hinze

7920 Norfolk Ave.

Bethesda, MD 20814

Defense Nuclear Facilities Safety

Board

Attn: D. Winters

625 Indiana Ave. NW

Suite 700

Washington, DC 20004

Nuclear Waste Technical Review Board

Attn: Library (2)

1100 Wilson Blvd.

Suite 910

Arlington, VA 22209-2297

\section{State Agencies}

New Mexico Bureau of Mines and Mineral Resources

Socorro, NM 87801

New Mexico Energy, Minerals and Natural Resources Department

Attn: Librarian

2040 South Pacheco

Santa Fe, NM 87505

New Mexico Energy, Minerals and Natural Resources Department New Mexico Radioactive Task Force (2) (Governor's WIPP Task Force)

Attn: A. Lockwood, Chairman C. Wentz, Policy Analyst

2040 South Pacheco

Santa Fe, NM 87505

Bob Forrest

Mayor, City of Carlsbad

PO Box 1569

Carlsbad, NM 88221

Carlsbad Department of Development Executive Director

Attn: C. Bernard

PO Box 1090

Carlsbad, NM 88221
New Mexico Environment Department Secretary of the Environment (3)

Attn: J. Espinosa

PO Box 968

1190 St. Francis Drive

Santa Fe, NM 87503-0968

New Mexico Environment Department

Attn: P. McCasland

WIPP Project Site Office

PO Box 3090

Carlsbad, NM 88221-3090

New Mexico State Engineer's Office Attn: M. Chudnoff

PO Box 25102

Santa Fe, NM 87504-5102

Environmental Evaluation Group (5) Attn: R. Neill

7007 Wyoming Blvd. NE, Suite F-2

Albuquerque, NM 87109

Inyo County Planning Department

Attn: Brad Mettam

Drawer L

Independence, CA 93526

\section{Advisory Committee on Nuclear Facility Safety}

John F. Ahearne

Executive Director, Sigma Xi

99 Alexander Drive

Research Triangle Park, NC 27709

James E. Martin

109 Observatory Road

Ann Arbor, MI 48109

\section{WIPP Committee, National Research Council's} Board on Radioactive Waste Management

National Research Council (2)

Board on Radioactive Waste Management

Attn: C.A. Anderson

I.B. Alterman

2101 Constitution Ave. NW

Harris Bldg. HA 456

Washington, DC 20418 
Howard Adler

Oxyrase, Inc.

11020 Solway School Rd.

Knoxville, TN 37931

John 0. Blomeke

3833 Sandy Shore Drive

Lenoir City, TN 37771-9803

John D. Bredehoeft

Western Region Hydrologist

Water Resources Division

US Geological Survey (M/S 439)

345 Middlefield Road

Menlo Park, CA 94025

Fred M. Ernsberger

1325 NW Tenth Ave.

Gainsville, FL 32605

Rodney C. Ewing

Department of Geology

University of New Mexico

Albuquerque, NM 87131

Charles Fairhurst

Department of Civil and Mineral

Engineering

University of Minnesota

500 Pillsbury Dr. SE

Minneapolis, MN 55455-0220

B. John Garrick

PLG, Incorporated

4590 MacArthur Blvd., Suite 400

Newport Beach, CA 92660-2027

Leonard F. Konikow

US Geological Survey

431 National Center

Reston, VA 22092

Jeremiah O'Driscoll

Jody, Incorporated

505 Valley Hill Drive

Atlanta, GA 30350

Christopher G. Whipple

ICF Kaiser Engineers

1800 Harrison St. 7th Floor

Oakland, CA 94612-3430
Thomas A. Zordan

Zordan Associates, Inc.

3807 Edinburg Dr.

Murrysville, PA 15668

\section{Performance Assessment Peer Review Panel}

G. Ross Heath

College of Ocean \& Fishery Sciences

University of Washington

583 Henderson $\mathrm{Hall}$, $\mathrm{HN}-15$

Seattle, WA 98195

Thomas H. Pigford

Department of Nuclear Engineering

4159 Etcheverry Hall

University of California

Berkeley, CA 94720

Thomas A. Cotton

JK Research Associates, Inc.

4429 Butterworth Place NW

Washington, DC 20016

Robert J. Budnitz

President, Future Resources

Associates, Inc.

2000 Center Street

Suite 418

Berkeley, CA 94704

C. John Mann

Department of Geology

245 Natural History Bldg.

1301 West Green Street

University of Illinois

Urbana, IL 61801

Frank W. Schwartz

Department of Geology and Mineralogy

The Ohio State University

Scott Hall

1090 Carmack Rd.

Columbus, $\mathrm{OH} 43210$

\section{National Laboratories}

Argonne National Laboratory (2)

Attn: A. Smith

D. Tomasko

9700 South Cass, Bldg. 201

Argonne, IL 60439 
Battelle Pacific Northwest

Laboratory (2)

Attn: S. Bates

R.E. Westerman

MSIN P8-44

Battelle Boulevard

Richland, WA 99352

Idaho National Engineering

Laboratory (2)

Attn: H. Loo

R. Klinger

Mail Stop 5108

Idaho Falls, ID 83403-4000

Los Alamos National Laboratory (5)

Attn: B. Erdal, INC-12

M. Ennis, HS-12

Mail Stop J900

S. Kosiewicz, EM-7

Mail Stop J595

L. Soholt, EM-13 Mail Stop M992

$\mathrm{J}$. Wenzel, HS-12

Mail Stop $\mathrm{K} 482$

PO Box 1663

Los Alamos, NM 87545

Oak Ridge National Laboratory

Transuranic Waste Manager

Attn: D.W. Turner

Bldg. 3047

PO Box 2008

Oak Ridge, TN 37831-6060

Pacific Northwest Laboratory

Attn: B. Kennedy

PO Box 999

Richland, WA 99352

Westinghouse-Savannah River

Technology Center (4)

Attn: N. Bibler

J.R. Harbour

M.J. Plodinec

G.G. Wicks

Aiken, SC 29802

\section{Corporations/Members of the Public}

Battelle Memorial Institute

Attn: R. Root

J. Kircher

505 Marquette NW, Suite 1

Albuquerque, NM 87102
Benchmark Environmental Corp. Attn: C. Frederickson

4501 Indian School NE, Suite 105

Albuquerque, NM 87110

Beta Corporation Int.

Attn: E. Bonano

6613 Esther NE

Albuquerque, NM 87109

City of Albuquerque

Public Works Department

Utility Planning Division

Attn: W.K. Summers

PO Box 1293

Albuquerque, NM 87103

Deuel and Associates, Inc.

Attn: R.W. Prindle

7208 Jefferson NE

Albuquerque, NM 87109

Disposal Safety, Inc.

Attn: B. Ross

1660 L Street NW, Suite 314

Washington, DC 20036

Ecodynamics (2)

Attn: P. Roache

R. Blaine

PO Box 9229

Albuquerque, NM 87119-9229

EG \& G Idaho (3)

1955 Fremont Street

Attn: C. Atwood

C. Hertzler

T.I. Clements

Idaho Falls, ID 83415

Geomatrix

Attn: K. Coppersmith

100 Pine Si., Suite 1000

San Francisco, CA 94111

Golder Associates, Inc.

Attn: R. Kossik

4104 148th Avenue NE

Redmond, WA 98052

John Hart and Associates, P.A.

Attn: J.S. Hart

2815 Candelaria Road NW

Albuquerque, NM 87107 
John Hart and Associates, P.A. Attn: K. Lickliter

400-C 8th St. NW

Tacoma, WA 98439

INTERA, Inc.

Attn: A.M. LaVenue

1650 University Blvd. NE, Suite 300

Albuquerque, NM 87102

INTERA, Inc.

Attn: J.F. Pickens

6850 Austin Center Blvd., Suite 300

Austin, TX 78731

INTERA, Inc.

Attn: W. Stensrud

PO Box 2123

Carlsbad, NM 88221

INTERA, Inc.

Attn: W. Nelson

101 Convention Center Dr.

Suite 540

Las Vegas, NV 89109

IT Corporation (2)

Attn: R.F. McKinney

$\mathrm{J}$. Myers

Regional Office, Suite 700

5301 Central Avenue NE

Albuquerque, NM 87108

Lawrence Berkeley Laboratory

Attn: J. Long

Building $50 \mathrm{E}$

Berkeley, CA 94720

MAC Technical Services Co.

Attn: D.K. Duncan

8418 Zuni Road SE, Suite 200

Albuquerque, NM 87108

Newman and Holtzinger

Attn: C. Mallon

1615 L Street NW, Suite 1000

Washington, DC 20036

RE/SPEC, Inc. (2)

Attn: W. Coons

4775 Indian School NE, Suite 300

Albuquerque, NM 87110
$\mathrm{RE} / \mathrm{SPEC}$, Inc.

Attn: J.L. Ratigan

PO Box 725

Rapid City, SD 57709

Reynolds Electric and Engineering

Company, Inc.

Attn: E.W. Kendall

Building 790

Warehouse Row

PO Box 98521

Las Vegas, NV 89193-8521

SAIC

Attn: H.R. Pratt

10260 Campus Point Drive

San Diego, CA 92121

SAIC

Attn: C.G. Pflum

101 Convention Center $\mathrm{Dr}$.

Las Vegas, NV 89109

SAIC (3)

Attn: M. Davis

R. Guzowski

J. Tollison

2109 Air Park Road SE

Albuquerque, NM 87106

SAIC (2)

Attn: J. Young

D. Lester

18706 North Creek Parkway, Suite 110

Bothe11, WA 98011

Southwest Research Institute

Nuclear Waste Regulatory Analysis (2)

Attn: P.K. Nair

6220 Culebra Road

San Antonio, TX 78228-0510

Systems, Science, and Software (2)

Attn: E. Peterson

$P$. Lagus

Box 1620

La Jolla, CA 92038

TASC

Attn: S.G. Oston

55 Walkers Brook Drive

Reading, MA 01867 
Tech Reps, Inc. (8)

Attn: J. Chapman

C. Crawford

D. Marchand

T. Peterson

J. Stikar

D. Scott

M. Minahan

V. Gilliland

5000 Marble NE, Suite 222

Albuquerque, NM 87110

Tolan, Beeson \& Associates

Attn: T.L. Tolan

2320 W. 15th Avenue

Kennewick, WA 99337

TRW Environmental Safety Systems (2)

Attn: I. Sacks, Suite 800

L. Wildman, Suite 1300

2650 Park Tower Drive

Vienna, VA 22180-7306

Sanford Cohen and Associates

Attn: J. Channell

7101 Carriage Rd NE

Albuquerque, NM 87109

Westinghouse Electric Corporation (5)

Attn: Library

C. Cox

L. Fitch

B.A. Howard

R.F. Kehrman

PO Box 2078

Carlsbad, NM 88221

Westinghouse Hanford Company

Attn: D.E. Wood, MSIN HO-32

PO Box 1970

Richland, WA 99352

Western Water Consultants

Attn: P.A. Rechard

PO Box 4128

Laramie, WY 82071

Western Water Consultants

Attn: D. Fritz

1949 Sugarland Drive \#134

Sheridan, WY 82801-5720
P. Drez

8816 Cherry Hills Road NE

Albuquerque, NM 87111

David Lechel

9600 Allende Rd. NE

Albuquerque, NM 87109

C.A. Marchese

PO Box 21790

Albuquerque, NM 87154

Arend Meijer

3821 Anderson SE

Albuquerque, NM 87108

D.W. Powers

Star Route Box 87

Anthony, TX 79821

Shirley Thieda

PO Box 2109, RR1

Bernalillo, NM 87004

Jack Urich

c/o CARD

144 Harvard SE

Albuquerque, NM 87106

\section{Universities}

University of California

Mechanical, Aerospace, and

Nuclear Engineering Department (2)

Attn: W. Kastenberg

D. Browne

5532 Boelter Hall

Los Angeles, CA 90024

University of California

Engineering and Applied Science

Attn: D. Okrent

48-121A Engineering IV

Los Angeles, CA 90024-1597

University of California

Mine Engineering Department

Rock Mechanics Engineering

Attn: N. Cook

Berkeley, CA 94720 
University of Hawaii at Hilo

Business Administration

Attn: S. Hora

Hilo, HI 96720-4091

University of Illinois

Department of Geology

Attn: C. Bethke

1301 W. Green St.

Urbana, IL 61801

University of New Mexico

Geology Department

Attn: Library

Albuquerque, NM 87131

University of New Mexico

Research Administration

Attn: H. Schreyer

102 Scholes Hall

Albuquerque, NM 87131

University of Wyoming

Department of Civil Engineering

Attn: V.R. Hasfurther

Laramie, WY 82071

University of Wyoming

Department of Geology

Attn: J.I. Drever

Laramie, WY 82071

University of Wyoming

Department of Mathematics

Attn: R.E. Ewing

Laramie, WY 82071

\section{Libraries}

Thomas Brannigan Library

Attn: D. Dresp

$106 \mathrm{~W}$. Hadley St.

Las Cruces, NM 88001

New Mexico State Library

Attn: N. McCallan

325 Don Gaspar

Santa Fe, NM 87503

New Mexico Tech

Martin Speere Memorial Library

Campus Street

Socorro, NM 87810
New Mexico Junior College

Pannell Library

Attn: R. Hill

Lovington Highway

Hobbs, NM 88240

Carlsbad Municipal Library

WIPP Public Reading Room

Attn: L. Hubbard

$101 \mathrm{~S}$. Halagueno St.

Carlsbad, NM 88220

University of New Mexico

Zimmerman Library

Government Publications Department

Albuquerque, NM 87131

\section{NEA/Performance Assessment Advisory Group (PAAG)}

P. Duerden

ANSTO

Lucas Heights Research Laboratories

Private Mail Bag No. 1

Menai, NSW 2234

AUSTRALIA

Gordon S. Linsley

Division of Nuclear Fuel Cycle and Waste Management

International Atomic Energy Agency

PO Box 100

A-1400 Vienna, AUSTRIA.

Nicolo Cade11i

Commission of European Communities

200, Rue de la Loi

B-1049 Brussels, BELGIUM

R. Heremans

Organisme Nationale des Déchets

Radioactifs et des Matiéres Fissiles (ONDRAF)

Place Madou 1, Boitec 24/25

B-1030 Brussels, BELGIUM

J . Marivoet

Centre d'Etudes de l'Energie

Nucléaire (CEN/SCK)

Boeretang 200

B-2400 Mol, BELGIUM 
P. Conlon

Waste Management Division

Atomic Energy Control Board (AECB)

PO Box 1046

Ottawa, Ontario KIP 559, CANADA

A.G. Wikjord

Manager, Environmental and Safety

Assessment Branch

Atomic Energy of Canada Limited

Whiteshel1 Research Establishment

Pinawa, Manitoba ROE 1LO, CANADA

Teollisuuden Voima Oy (TVO) (2)

Attn: Timo Äikäs Jukka-Pekka Salo

Annankatu $42 \mathrm{C}$

SF-00100 Helsinki Suomi, FINLAND

Timo Vieno

Technical Research Centre of Finland

(VTT)

Nuclear Energy Laboratory

PO Box 208

SF-02151 Espoo, FINLAND

Division de la Sécurité et de la

Protection de l'Environment (DSPE)

Commissariat á l'Energie Atomique

Agence Nationale pour la Gestion des

Déchets Radioactifs (ANDRA)

Attn: Gérald Ouzounian

M. Claude Ringeard

Route du Panorama Robert Schuman

B. P. No. 38

F-92266 Fontenay-aux-Roses Cedex

FRANCE

Claudio Pescatore

Division of Radiation Protection and

Waste Management

OECD Nuclear Energy Agency

38, Boulevard Suchet

F-75016 Paris

FRANCE

M. Dominique Greneche

Commissariat á 1'Energie Atomique

IPSN/DAS/SASICC/SAED

B.P. No. 6

F-92265 Fontenay-aux-Roses Gedex

FRANCE
Robert Fabriol

Bureau de Recherches Géologiques et Miniéres (BRGM)

B.P. 6009

45060 Orléans Cedex 2

FRANCE

P. Bogorinski

Gesellschaft für Reaktorsicherheit (GRS) MBH

Schwertnergasse 1

D-5000 Köln 1, GERMANY

R. Storck

GSF - Institut für Tieflagerung

Theodor-Heuss-Strabe 4

D-3300 Braunschweig, GERMANY

Ferrucio Gera

ISMES S.P.A

Via del Crociferi 44

I-00187 Rome, ITALY

Hiroyuki Umeki

Isolation System Research Program

Radioactive Waste Management Project

Power Reactor and Nuclear Fuel

Development Corporation (PNC)

1-9-13, Akasaka, Minato-ku

Tokyo 107

JAPAN

Tönis Papp

Swedish Nuclear Fuel and Waste

Management Co.

Box 5864

S 10248 Stockholm

SWEDEN

Conny Hägg

Swedish Radiation Protection

Institute (SSI)

Box 60204

S-104 01 Stockholm, SWEDEN

J. Hadermann

Paul Scherrer Institute

Waste Management Programme

CH-5232 Villigen PSI

SWITZERIAND 
J. Vigfusson

HSK-Swiss Nuclear Safety Inspectorate Federal Office of Energy

CH-5232 Villigen-HSK

SWITZERLAND

D.E. Billington

Departmental Manager-Assessment

Studies

Radwaste Disposal R\&D Division

AEA Decommissioning \& Radwaste

Harwel1 Laboratory, B60

Didcot Oxfordshire OXII ORA

UNITED KINGDOM

P. Grimwood

Waste Management Unit

BNFL

Sellafield

Seascale, Cumbria CA20 1PG

UNITED KINGDOM

Alan J. Hooper

UK Nirex Ltd

Curie Avenue

Harwell, Didcot

Oxfordshire, OX11 ORH

UNITED KINGDOM

Jerry M. Boak

Yucca Mountain Project Office

US Department of Energy

PO Box 98608

Las Vegas, NV 89193

Seth M. Coplan (Chairman)

US Nuclear Regulatory Commission

Division of High-Level Waste

Management

Mail Stop 4-H-3

Washington, DC 20555

A.E. Van Luik

INTERA/M\&O

The Valley Bank Center

101 Convention Center Dr.

Las Vegas, NV 89109
NEA/Probabilistic System Assessment Group (PSAG)

Shaheed Hossain

Division of Nuclear Fuel Cycle and Waste Management

International Atomic Energy Agency Wagramerstrasse 5

PO Box 100

A-1400 Vienna, AUSTRIA

Alexander Nies (PSAC Chairman)

Gesellschaft für Strahlen- und

Institut für Tieflagerung

Abteilung für Endlagersicherheit

Theodor-Heuss-Strasse 4

D-3300 Braunschweig

GERMANY

Eduard Hofer

Gesellschaft für Reaktorsicherheit (GRS) MBH

Forschungsgelände

D-8046 Garching, GERMANY

Andrea Saltelli

Commission of the European

Communities

Joint Resarch Centre of Ispra

I-21020 Ispra (Varese)

ITALY

Alejandro Alonso

Gátedra de Tecnologia Nuclear

E.T.S. de Ingenieros Industriales

José Gutiérrez Abascal, 2

E-28006 Madrid, SPAIN

ENRESA (2)

Attn: M. A. Cuñado

F. J. Elorza

Calle Emilio Vargas, 7

E-28043 Madrid, SPAIN

Pedro Prado

CIEMAT

Instituto de Tecnología Nuclear

Avenida Complutense, 22

E-28040 Madrid, SPAIN 
Nils A. Kjellbert

Swedish Nuclear Fuel and Waste

Management Company (SKB)

Box 5864

S-102 48 Stockholm, SWEDEN

Björn Cronhjort

Royal Institute of Technology

Automatic Control

S-100 44 Stockholm, SWEDEN

Richard A. Klos

Paul-Scherrer Institute (PSI)

CH-5232 Villingen PSI, SWITZERLAND

Nationale Genossenschaft für die

Lagerung Radioaktiver Abfälle (2)

Attn: C. McCombie

F. Van Dorp

Hardstrasse 73

CH-5430 Wettingen, SWITZERLAND

N. A. Chapman

Intera Information Technologies

Park View House

14B Burton Street

Melton Mowbray

Leicestershire LE13 IAE

UNITED KINGDOM

Daniel A. Galson

Galson Sciences Ltd.

35, Market Place

Oakham

Leicestershire LE15 6DT

UNITED KINGDOM

David P. Hodgkinson

Intera Information Technologies

45 Station Road, Chiltern House

Henley-on-Thames

Oxfordshire RG9 1AT

UNITED KINGDOM

Brian G.J. Thompson

Department of the Environment: Her

Majesty's Inspectorate of Pollution

Room A5.33, Romney House

43 Marsham Street

London SW1P 2PY, UNITED KINGDOM
Intera Information Technologies

Attn: M.J.Apted

3609 South Wadsworth Blvd.

Denver, CO 80235

US Nuclear Regulatory Commission (2)

Attn: R. Codell

N. Eisenberg

Mail Stop 4-H-3

Washington, DC 20555

Battelle Pacific Northwest

Laboratories

Attn: P.W. Eslinger

MS K2-32

PO Box 999

Richland, WA 99352

Center for Nuclear Waste Regulatory

Analysis (CNWRA)

Southwest Research Institute

Attn: B. Sagar

PO Drawer 28510

6220 Culebra Road

San Antonio, TX 78284

\section{Geostatistics Expert Working Group (GXG)}

Rafael L. Bras

R.L. Bras Consulting Engineers

44 Percy Road

Lexington, MA 02173

Jesus Carrera

Universidad Politécnica de Cataluña E.T.S.I. Caminos

Jordi, Girona 31

E-08034 Barcelona, SPAIN

Gedeon Dagan

Department of Fluid Mechanics and

Heat Transfer

Tel Aviv University

Po Box 39040

Ramat Aviv, Tel Aviv 69978

ISRAEL 
Ghislain de Marsily (GXG Chairman) University Pierre et Marie Curie Laboratorie de Geologie Applique

4, Place Jussieu

T.26 - 5 e etage

75252 Paris Cedex 05

FRANCE

Alain Galli

Centre de Geostatistique

Ecole des Mines de Paris

35 Rue St. Honore

77035 Fontainebleau, FRANCE

Christian Ravenne

Geology and Geochemistry Division

Institut Francais du Pétrole

$1 \& 4$, Av. de Bois-Préau B.P. 311

92506 Rueil Malmaison Cedex

FRANCE

Peter Grindrod

INTERA Information Technologies Ltd.

Chiltern House

45 Station Road

Henley-on-Thames

Oxfordshire, RG9 IAT, UNITED KINGDOM

Alan Gutjahr

Department of Mathematics

New Mexico Institute of Mining and

Technology

Socorro, NM 87801

C. Peter Jackson

Harwell Laboratory

Theoretical Studies Department

Radwaste Disposal Division

Bldg. 424.4

Oxfordshire Didcot Oxon OXII ORA

UNITED KINDGOM

Rae Mackay

Department of Civil Engineering

University of Newcastle Upon Tyne

Newcastle Upon Tyne NE1 7RU

UNITED KINGDOM

Steve Gorelick

Department of Applied Earth Sciences Stanford University

Stanford, CA 94305-2225
Peter Kitanidis

60 Peter Coutts Circle

Stanford, CA 94305

Dennis McLaughlin

Parsons Laboratory

Room 48-209

Department of Civil Engineering

Massachusetts Institute of Technology

Cambridge, MA 02139

Shlomo P. Neuman

College of Engineering and Mines

Hydrology and Water Resources Dept.

University of Arizona

Tucson, AZ 85721

Yoram Rubin

Department of Civil Engineering

University of California

Berkeley, CA 94720

\section{Future Societies Expert Panel}

Michael Baram

Bracken and Baram

33 Mount Vernon St.

Bolton, MA 02108

Wendell Bell

Department of Sociology

Yale University

1965 Yale Station

New Haven, CT 06520

Gregory Benford

University of California, Irvine

Department of Physics

Irvine, CA 92717

Duane Chapman

210 Warren Hall

Department of Agricultural Economics

Cornell University

Ithaca, NY 14853-7801

Bernard L. Cohen

Department of Physics

University of Pittsburgh

Pittsburgh, PA 15260 
Victor Ferkiss

23 Sage Brush Circle

Corrales, NM 87048

Theodore S. Glickman

Resources for the Future

1616 P St., NW

Washington, DC 20036

Ted Gordon

The Futures Group

80 Glastonbury Blvd.

Glastonbury, GT 06033

Craig Kirkwood

College of Business Administration

Arizona State University

Tempe, AZ 85287

Harry Otway

Health, Safety, and Envir. Div.

Mail Stop K-491

Los Alamos National Laboratory

Los Alamos, NM 87545

Martin J. Pasqualetti

Department of Geography

Arizona State University

Tempe, AZ 85287-3806

Dan Reicher

US Department of Energy

$S-1$

1000 Independence Ave, SW

Washington, DC 20585

Norman Rosenberg

Resources for the Future

1616 P St., NW

Washington, DC 20036

Max Singer

the Potomac Organization, Inc.

5400 Greystone St.

Chevy Chase, MD 20815

Theodore Taylor

P.0. Box 39

3383 Weatherby Rd.

West Clarksville, NY 14786
Maris Vinovskis

Institute for Social Research

Room 4086

University of Michigan

426 Thompson St.

Ann Arbor, MI 48109-1045

\section{Marker Development \\ Expert Panel}

Dieter G. Ast

Department of Materials Science

Bard Hall

Cornell University

Ithaca, NY 14853-1501

Victor R. Baker

Department of Geosciences

Building \#77, Gould-Simpson Building

University of Arizona

Tucson, AZ 85721

Michael Brill

President

BOSTI

1479 Hertel Ave.

Buffalo, NY 14216

Frank D. Drake

Board of Studies in Astronomy and

Astrophysics

Lick Observatory

University of California, Santa Cruz Santa Cruz, CA 95064

Ben R. Finney

University of Hawaii at Manoa

Department of Anthropology

Porteus Hall 346, 2424 Maille Way

Honolulu, HI 96822

David B. Givens

American Anthropological Association 4350 N. Fairfax Drive, Suite 640

Arlington, VA 22203

Ward H. Goodenough

Department of Anthropology

University of Pennsylvania

325 University Museum

33 rd and Spruce Streets

Philadelphia, PA 19104-6398 
Maureen F. Kaplan

Eastern Research Group, Inc.

100 Hartwell Avenue

Lexington, MA 02173

Jon Lomberg

P.0. Box 207

Honaunau, HI 967626

Louis E. Narens

Department of Cognitive Sciences

School of Social Sciences

University of California, Irvine

Irvine, CA 92717

Frederick J. Newmeyer

Department of Linguistics

GN-40

University of Washington

Seattle, WA 98195

Woodruff T. Sullivan, III

Department of Astronomy

FM-20

University of Washington

Seattle, WA 98195

Wendell S. Williams

Materials Science and Engineering

White Building

Case Western Reserve University

Cleveland, $\mathrm{OH} 44106$

\section{Individuals}

Jill Bartel

6129C Shanda Drive

Raleigh, NC 27609

Michael A. Bauser

Neuman \& Holtzinger

1615 L St, NW

Suite 1000

Washington, DC 20036

Paul Carroll

U.S. Congress

Office of Technology Assessment

Ocean and Environment Program

Washington, DC 20510-8025
Richard Clegg

British Nuclear Fuels, plc

Building B433

Sellafield Seascale Cumbria

CA20 1PG

UNITED KINGDOM

Matthew Coolidge

499 Embarcadero

Oakland, CA 94606

Jeff Davis

Mother Jones Magazine

1663 Mission Street

San Francisco, CA 94103

Thomas W. Fogwell

International Technology Corporation 4585 Pacheco Boulevard

Martinez, CA 94553

Don Hancock

Southwest Research and Information Center

P.O. Box 4524

Albuquerque, NM 87106

Randy Hanson

3620 15th Avenue South

Minneapolis, MN 55407

W.C. Kirwin

Department of Fine Arts

University of Guelph

Guelph

Province of Ontario

CANADA N1G 2W1

Joe Knap

TRICAN

347 Greenwich St.

Brantford, Ontario

N3P 5T3

CANADA

Donald Lipski

1061 Manhattan Ave.

Brooklyn, NY 11222 
Timothy Margulies

USEPA

Mid Code $6602 \mathrm{~J}$

401 M St. , NW

Washington, D.C. 20460

Steven R. Mattson

Science Applications International

Gorporation

Valley Bank Center

101 Convention Center Drive

Las Vegas, NV 89109

Elizabeth R. Mertz

The Carnegie Museum of Natural History

Publications

4400 Forbes Avenue

Pittsburgh, PA 15213-4080

Ardyce Milton

Clark County Nuclear Waste Division

301 E. Clark Avenue, Suite 570

Las Vegas, NV 89101

Homi Minwalla

Roy $T$. Weston, Inc.

955 L'Enfant Plaza, SW

Eighth Floor

Washington, D.C. 20024

Jim Morris

Design Department

SUNY College at Buffalo

1300 Elmwood Ave.

Buffalo, NY 14222

Mike O'Neil

U.S. Bureau of Land Management

New Mexico State Office

1474 Rodeo Road

Santa Fe, NM 87502

David Okrent

439 Veteran Avenue

Los Angeles, CA 90024

Ken Peterson

Westinghouse Hanford Company

P.O. Box 1970, H4-14

Richland, WA 99352
Catherine Rich

University of California

at Los Angeles

Department of Geography

405 Hilgard Avenue

Los Angeles, CA 90024-1524

Kyle Rogers

USEPA

Office of Radiation Programs

Mail Code ANR-460

401 M Street, SW

Washington, DC 20460

Tim Rohrer

1350 West Hualapai

Tucson, AZ 85745

Patricia Rosati (2)

St. Martin's Press, Inc.

Permissions

175 Fifty Avenue

New York, NY 10010-7848

Carl Sagan

Space Sciences Building

Cornell University

Ithaca, NY 14850

Al Schenker

Los Alamos Technical Associates, Inc.

2400 Louisiana Blvd., NE

Building 1, Suite 400

Albuquerque, NM 87110

Steve Schwartz

Military Production Network

218 D Street, SE

2nd Floor

Washington, DC 20003

Terry Simmons

403 Flint Sireet

Reno, NV 89501

Rolf Sjöblom

Swedish Nuclear Power Inspectorate

Sehlstedtsgatan 11

Box 27106

S-102 52 Stockholm

SWEDEN 
Gar Smith

Earth Island Journal

300 Broadway, Suite 28

San Francisco, GA 94133-3312

Chris Solloway

U.S. EPA

PM-223

402 M Street, SW

Washington, DC 20460

George Stromeyer

Ascot Square 1603

3850 Woodhaven Road

Philadelphia, PA 19154

David R. Turner

Southwest Research Institute 6220 Culebra Road

San Antonio, TX 78238-5166

Joel Weishaus

2812 Garfield, SE \#E

Albuquerque, NM 87106

Simon J. Wisbey

AEA Technology

B60, Harwel1 Laboratory

Didcot

Oxfordshire OXII ORA

UNITED KINGDOM

Jya-Syin Wu

38-137 ENG-IV

University of California at

Los Angeles

Los Angeles, CA 90024

\section{Foreign Addresses}

Studiecentrum Voor Kernenergie

Centre D'Energie Nucleaire

Attn: A. Bonne

SCK/CEN

Boeretang 200

B-2400 MoI, BELGIUM

Atomic Energy of Canada, Ltd. (3)

Whiteshell Research Establishment

Attn: M.E. Stevens

B.W. Goodwin

D. Wushke

Pinewa, Manitoba ROE 1LO, CANADA
Juhani Vira

Teollisuuden Voima Oy (TVO)

Annankatu $42 \mathrm{C}$

SF-00100 Helsinki Suomi

FINLAND

Jean-Pierre Olivier

OECD Nuclear Energy Agency (2)

38, Boulevard Suchet

F-75016 Paris, FRANCE

D. Alexandre, Deputy Director

ANDRA

31 Rue de la Federation

75015 Paris, FRANCE

Claude Sombret

Centre D'Etudes Nucleaires

De Ia Vallee Rhone

CEN/VALRHO

S.D.H.A. B.P. 171

30205 Bagnols-Sur-Ceze, FRANCE

Bundesministerium fur Forschung und

Technologie

Postfach 200706

5300 Bonn 2, GERMANY

Bundesanstalt fur Geowissenschaften und Rohstoffe

Attn: M. Langer

Postfach 510153

3000 Hanover 51, GERMANY

Gesellschaft fur Reaktorsicherheit

(GRS) (2)

Attn: B. Baltes

W. Muller

Schwertnergasse 1

D-5000 Cologne, GERMANY

Institut fur Tieflagerung (2)

Attn: K. Kuhn

Theodor-Heuss-Strasse 4

D-3300 Braunschweig, GERMANY

Physikalisch-Technische

Bundesanstalt

Attn: P. Brenneke

Postfach 3345

D-3300 Braunschweig, GERMANY 
Shingo Tashiro

Japan Atomic Energy Research

Institute

Tokai-Mura, Ibaraki-Ken

319-11, JAPAN

Netherlands Energy Research

Foundation (ECN)

Attn: L.H. Vons

3 Westerduinweg

PO Box 1

1755 ZG Petten

THE NETHERLANDS

Johan Andersson

Swedish Nuclear Power Inspectorate

Statens Kärnkraftinspektion (SKI)

Box 27106

S-102 52 Stockholm

SWEDEN

Fred Karlsson

Svensk Kärnbränsleforsorjning $A B$

Project KBS

Box 5864

S-102 48 Stockholm

SWEDEN

Nationale Genossenschaft für die

Lagerung Radioaktiver Abfälle (2)

Attn: S. Vomvoris

P. Zuidema

Hardstrasse 73

CH-5430 Wettingen

SWITZERLAND

AEA Technology

Attn: J.E. Tinson

B4244 Harwell Laboratory

Didcot, Oxfordshire OX11 ORA

UNITED KINGDOM

AEA Technology

Attn: J.H. Rees

D5W/29 Gulham Laboratory

Abington.

Oxfordshire $\mathrm{OX14} 3 \mathrm{DB}$

UNITED KINGDOM

AEA Technology

Attn: W.R. Rodwell

044/A31 Winfrith Technical Centre

Dorchester

Dorset DT2 8DH, UNITED KINGDOM
D.R. Knowles

British Nuclear Fuels, plc

Risley, Warrington

Cheshire WA3 6AS, 1002607

UNITED KINGDOM

$\underline{\mathrm{MS}} \quad \underline{\mathrm{Org}}$.

$0101 \quad 1$

A. Narath

20 O.E. Jones

08271502 P.J. Homme

$0827 \quad 1511$ 'D.K. Gartling

01274511 D.P. Garber

07246000 D.L. Hartley

13246115 P.B. Davies

13246115 R.L. Beauheim

07506118 H.R. Westrich

13206119 E.D. Gorham

$1320 \quad 6119$ Staff (14)

13226121 J.R. Tillerson

13226121 Staff (7)

13376300 D.E. Ellis

13356302 L.E. Shephard

13356303 S.Y. Pickering

13356303 W.D. Weart

13356305 S.A. Goldstein

13356305 A.R. Lappin

13416306 A.L. Stevens

$1326 \quad 6312$ F.W. Bingham

13266313 L.S. Costin

13456331 P.A. Davis

6341 Sandia WIPP Central Files (150)

1328

1328

6342

D.R. Anderson

1328

1328

1341

1341

1341

1341

1341

1343

1330

0736

6342

Staff (30)

6343

6343

6345

V. Harper-Slaboszewicz

Staff (3)

6345

R.C. Lincoln

Staff (9)

6347

6348

D.R. Schafer

6348

J.T. Holmes

6351

6352

6400

Staff (4)

0746

6613

0746

6613

0746

0727

0718

0899

0619

1119

6613

6622

6641

7141

R.E. Thompson

S.E. Sharpton

N.R. Ortiz

R.M. Cranwell

R.L. Iman

C. Leigh

M.S.Y. Chu

R.E. Luna, Acting

Technical Library (5)

7151 Technical Publications

7613-2 Document Processing for DOE/OSTI (10)

8523-2 Central Technical Files 